DISEASE CHARACTERIZATION AND PRE-CLINICAL TESTING IN CANINE MODELS OF THE NEURONAL CEROID LIPOFUSCINOSES

A Dissertation
presented to
the Faculty of the Graduate School
at the University of Missouri-Columbia
In Partial Fulfillment
of the Requirements for the Degree
Doctor of Philosophy
Dr. Martin L. Katz, Dissertation Supervisor
JULY 2021
GRA ROBINSON KICK


The undersigned, appointed by the dean of the Graduate School, have examined the dissertation entitled

\title{
DISEASE CHARACTERIZATION AND PRE-CLINICAL TESTING IN CANINE MODELS OF THE NEURONAL CEROID LIPOFUSCINOSES
}

presented by Grace Robinson Kick, a candidate for the degree of Doctor of Philosophy, and hereby certify that, in their opinion, it is worthy of acceptance.

\author{
Dr. Martin L. Katz \\ Department of Ophthalmology \\ Dr. Rebecca E.H. Whiting \\ Department of Ophthalmology \\ Dr. Joan R. Coates \\ Department of Veterinary Medicine and Surgery \\ Dr. Elizabeth Bryda \\ Department of Veterinary Pathobiology \\ Dr. Lixing Reneker \\ Department of Ophthalmology
}




\section{ACKNOWLEDGEMENTS}

These projects have truly been a group effort and I am indebted to everyone who made them possible. First and foremost, l'd like to thank my mentor, Dr. Martin Katz, for his guidance and support over the last 5 years. I became a scientist under his supervision, and I'm deeply grateful for the resources, time and experience he shared with me.

I'd like to thank the members of my committee, Drs. Rebecca Whiting, Joan Coates, Lixing Reneker and Elizabeth Bryda. Drs. Whiting and Coates were directly involved in these studies. Dr. Whiting was a second mentor to me throughout this process and I am particularly indebted to her for my training in electroretinography and retinal imaging. Dr. Coates has been incredibly generous with her very limited time, sharing her considerable expertise to develop and implement several projects. Dr. Reneker always has advice to share. Dr. Bryda provided me with a fantastic opportunity to work as a TA, an experience that would otherwise have been sorely missed.

I was supported by a Life Sciences Fellowship financially, but the support of Debbie Allen and Dr. Mark Hannink that came along with it was invaluable.

The dogs who participated in these studies are in my opinion some of the happiest and most well-loved research dogs in the business, and that is due to Leilani Castaner and her undergraduate student workers as well as a succession of fantastic veterinarians: Drs. Daniella Vansteenkiste, Katherine Bibi, Stefanie Lim, Joe Kowal, Julianna Sabol and Elizabeth Meiman. I can't thank them 
enough for their care and compassion in working with the dogs, which has had a huge impact on how I think about the use of large animals in research, and more technically for their veterinary support and many hours spent in the dark during ERG recording sessions. Additional veterinary support was provided by Drs. Jackie Pearce, Juri Ota-Kuroki, Fred Wininger, Jeffrey Bryan, Dawna Voelkl and Dietrich Volkmann.

l'd like to thank the other members of the Neurodegenerative Diseases Research Laboratory, both past and present, who contributed this work. Cheryl Jensen processed samples and supplied huge quantities of excellent homegrown produce. Students Jeffrey Student, Jacob Taylor and Lauren Gillespie got me up and running in the lab when I first started for which I am incredibly grateful. I'd also like to thank Dr. Brandie Morgan-Jack for many very helpful problem-solving discussions.

Finally, thanks very much to my family - Nancy, Gregg \& Abbey Robinson and Daniel Kick - for all their love and support. My parents have always been both supportive and honest and told me I could do anything I put my mind to except surgery. Despite being the younger sister, Abbey has always given me excellent advice and, as she's also pursuing a PhD in biology, that went so far as to include reviewing parts of this dissertation for me ("and at times, it wasn't that dry!" being a highlight). Daniel is the best partner I could ask for and it's been great going through our PhDs together. 


\section{TABLE OF CONTENTS}

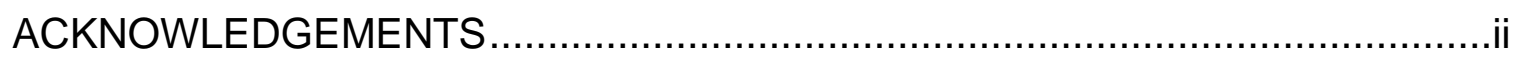

LIST OF FIGURES …............................................................................

LIST OF TABLES …..............................................................................

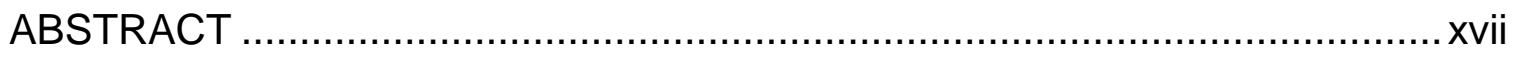

\section{CHAPTER}

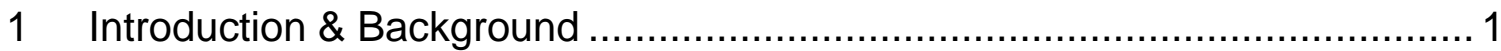

1.1 The Neuronal Ceroid Lipofuscinoses ............................................... 1

1.1.1 Neuronal Ceroid Lipofuscinoses in Humans ..................................... 1

1.1.2 Neuronal Ceroid Lipofuscinosis Type 2 (CLN2 Disease) .................... 3

1.1.3 The Canine Disease Model of CLN2 Disease ................................. 7

1.1.4 Neuronal Ceroid Lipofuscinosis Type 5 (CLN5 Disease) ................. 11

1.2 Approaches to Therapy for CLN2 Disease ..................................... 15

1.2.1 Enzyme Replacement Therapy ……...................................... 16

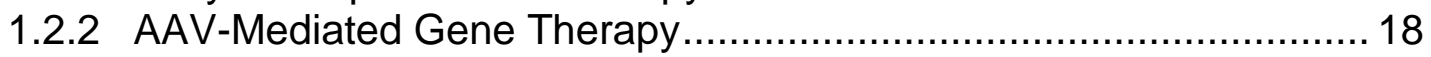

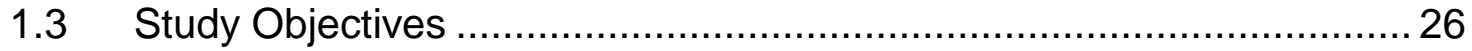

2 Intravitreal Enzyme Replacement Therapy Preserves Retinal Structure and

Function in the Canine Model of CLN2 Disease ..............................................29

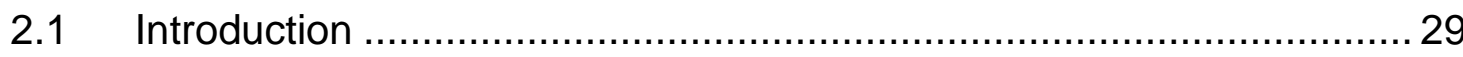

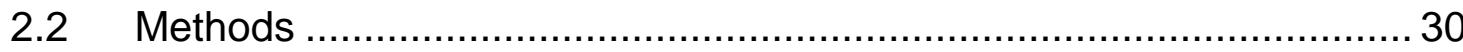

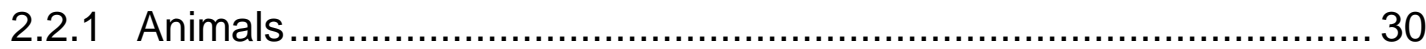

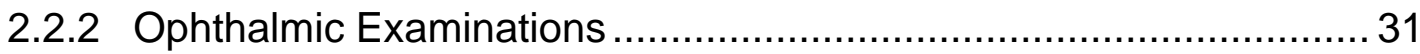

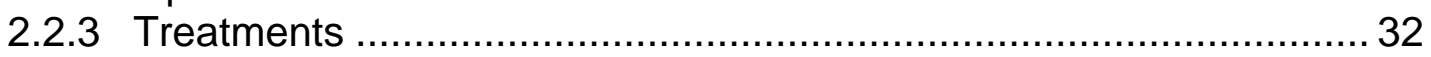

2.2.4 Immunosuppression and Inflammation Control .............................. 35

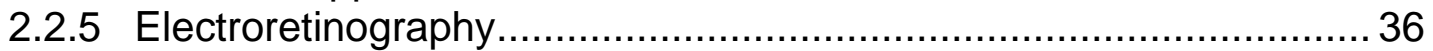

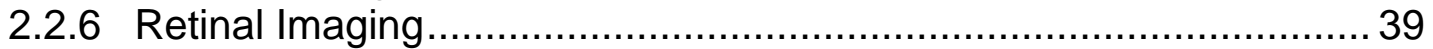

2.2.7 Euthanasia and Necropsy ........................................................ 40

2.2.8 Histology and Morphological Analysis ............................................. 41

2.2.9 Determination of rhTPP1 Concentration ....................................... 44

2.2.10 Identification of anti-TPP1 Antibodies ..................................... 46

2.2.11 Statistical Analysis ........................................................... 47

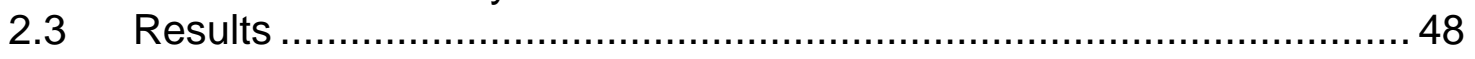

2.3.1 rhTPP1 Concentration in Aqueous Humor, Vitreous Humor and

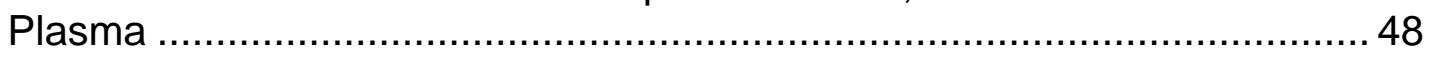

2.3.2 Ocular Inflammation.......................................................... 49

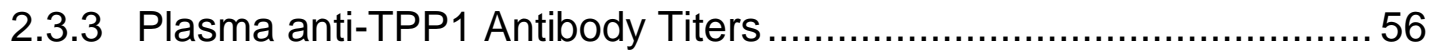

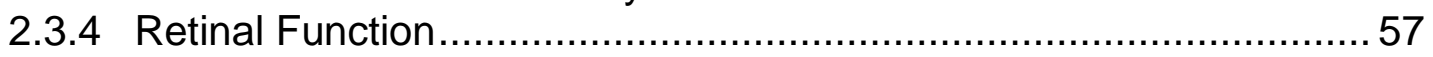

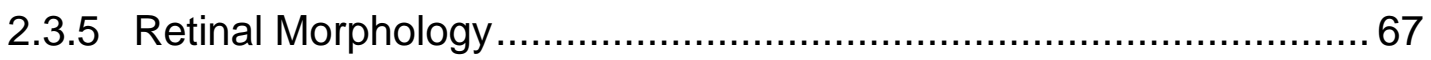

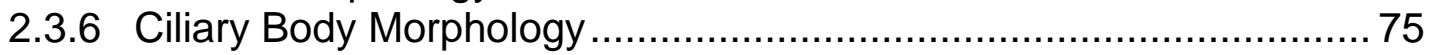


2.3.7 Indirect Assessment of Ganglion Cell Numbers ............................ 77

2.3.8 Retinal Ganglion Cell Storage Body Content ................................ 80

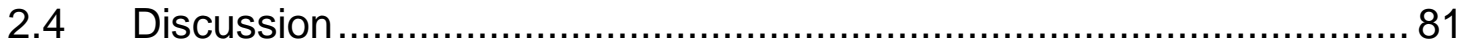

3 Implantation of TPP1-Overexpressing MSCs Preserves Retinal Function but did not Prevent Neurological Disease Progression........................................... 86

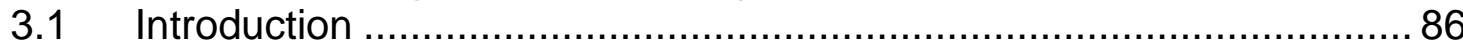

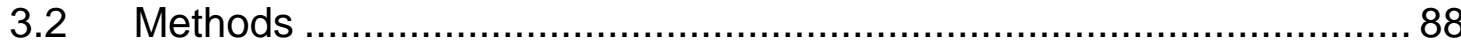

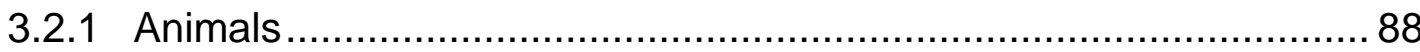

3.2.2 Neurologic and Ophthalmic Examinations ................................... 88

3.2.3 Isolation, Culture and Transduction of MSCs ................................. 89

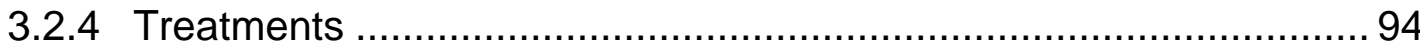

3.2.5 In vivo Measures to Assess Retinal Function and Structure ........... 100

3.2.6 MRI and Ventricular Volume Analysis......................................... 100

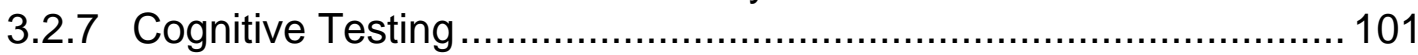

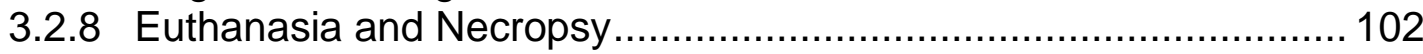

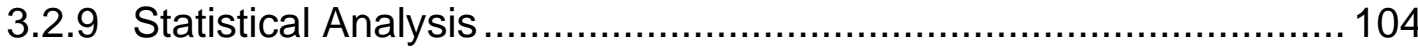

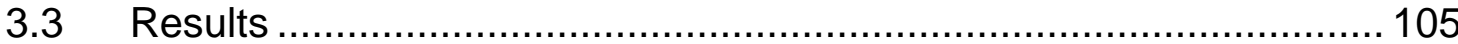

3.3.1 Detection of MSCs after Transplantation .................................... 105

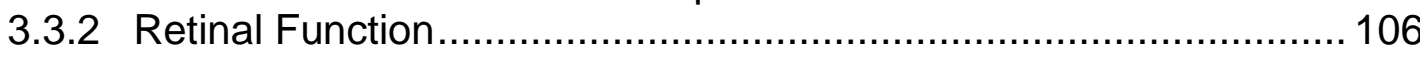

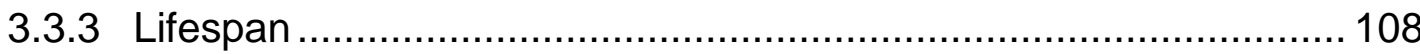

3.3.4 Onset of Neurologic Disease Signs ............................................... 109

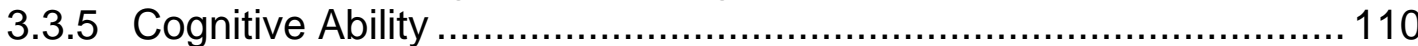

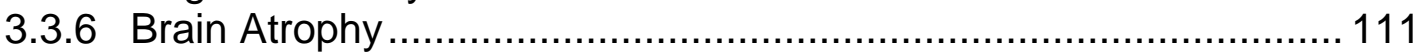

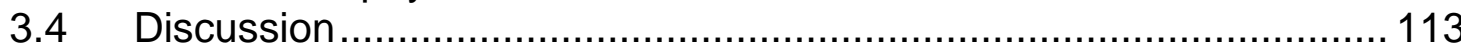

4 Intravitreal Gene Therapy Preserves Retinal Function in a Canine Model of CLN2 Neuronal Ceroid Lipofuscinosis ........................................................ 123

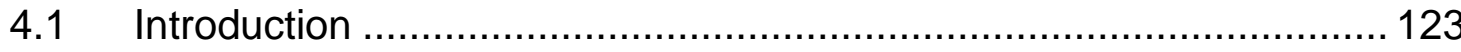

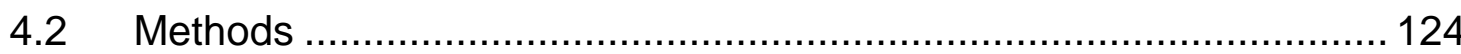

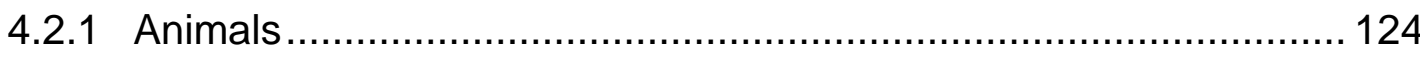

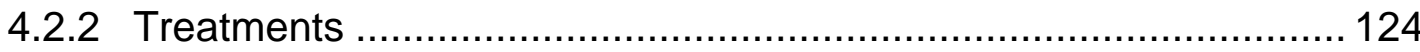

4.2.3 In vivo Measures to Assess Retinal Function and Structure ........... 130

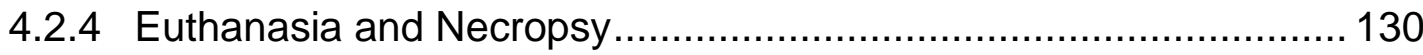

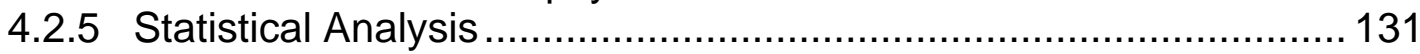

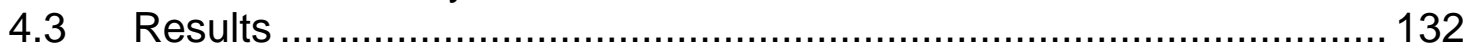

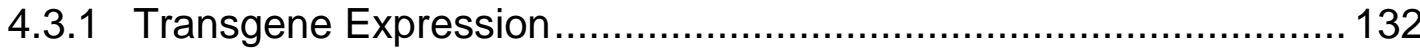

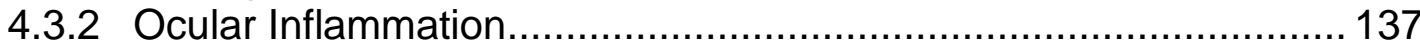

4.3.3 Retinal Function................................................................ 143

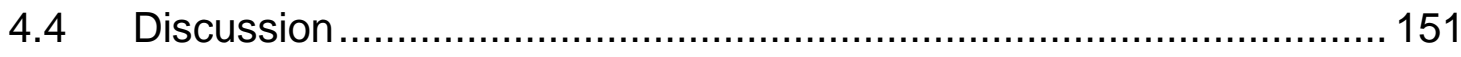

5 Characterization of Visual System Pathology in a Canine Model of CLN5 Neuronal Ceroid Lipofuscinosis .............................................................. 159

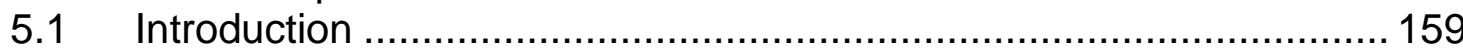

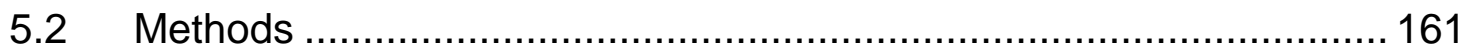

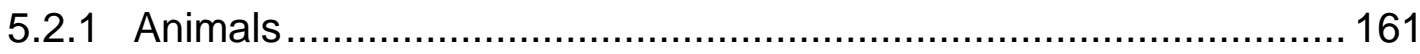

5.2.2 Electrophysiology and Retinal Imaging .................................... 161 


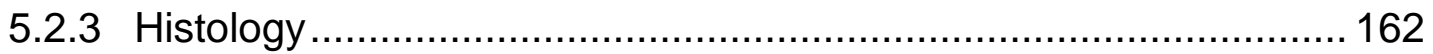

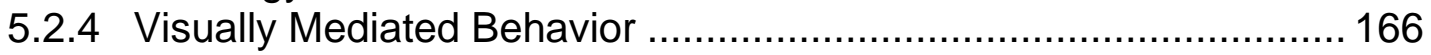

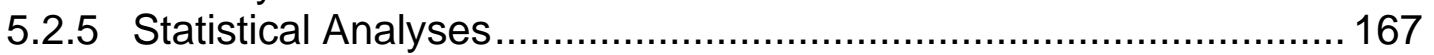

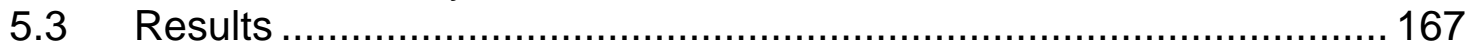

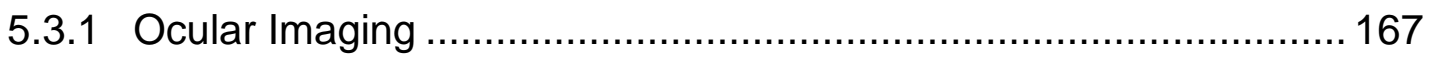

5.3.2 Electroretinographic and Visual Evoked Potential Findings ............ 169

5.3.3 Visually Mediated Behavior ..................................................... 173

5.3.4 Retinal and Occipital Cortex Morphology ..................................... 174

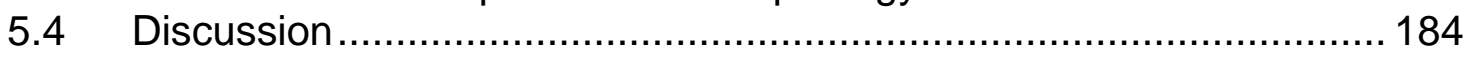

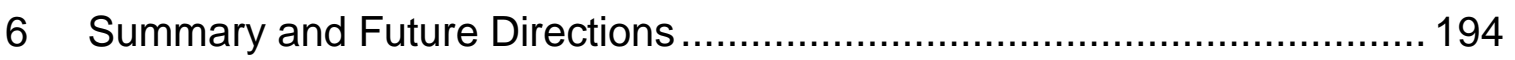

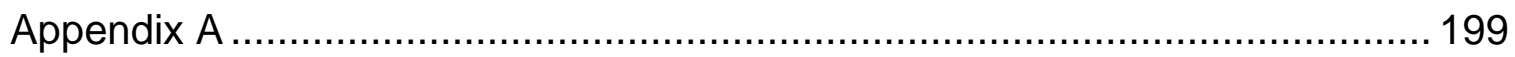

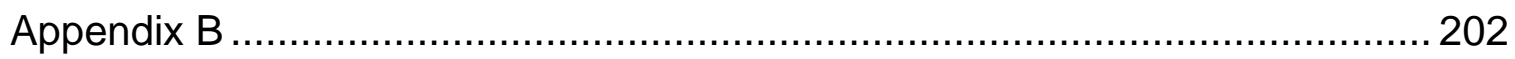

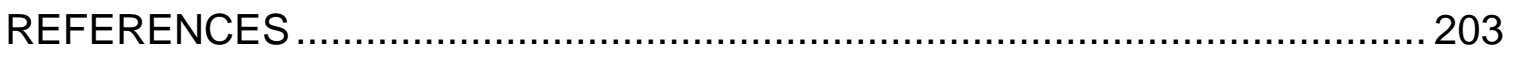

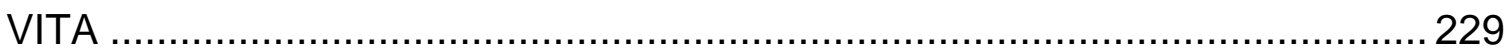




\section{LIST OF FIGURES}

Figure 1-1: TPP1 is trafficked to lysosomes via the M6P pathway; however, some newly synthesized TPP1 protein is instead secreted. Extracellular TPP1 can be taken up after binding to cell-surface M6P receptors and trafficked to the lysosome. Therefore, delivery of M6P-tagged TPP1 can correct the metabolic deficit in TPP1-deficient cells. The source of this extracellular TPP1 could be exogenously delivered recombinant protein or TPP1 secreted by a cell with a functional copy of TPP1.

Figure 2-1: Diagram of retinal dissection

Figure 2-2: Scanning laser ophthalmoscopy (SLO) fundus images from all early start dogs throughout the study. Inflammation that occurred early in the study resulted in long term vitreal opacity in treated eyes despite the resolution of active inflammation. By 10 months of age, dog E3 had developed widespread disease-related canine multifocal retinopathy lesions in the control eye but not in the treated eye.

Figure 2-3: SLO fundus images from all late start dogs at the end of the study. Images remained normal in all dogs following the initial dose, but dogs L2 and L4 exhibited intraocular inflammation following the second dose. While active inflammation gradually resolved, vitreal opacity persisted through the remainder of the study in both dogs (orange arrows). Final images for dog L1 were taken days prior to the second dose as follow-up images were not possible due to unscheduled euthanasia. By 10 months of age, dog L3 had developed widespread disease-related CMR lesions in the control eye (red arrows) but not in the treated eye.

Figure 2-4: Light micrographs of sections of the mid-peripheral rhTPP1-treated retinas from dogs L2 (A) and L3 (B). Pronounced perivascular cuffing was present around inner retinal vessels in the central retina of dog $\mathrm{L} 2$ (red arrows), whereas no such cuffing was present around inner retinal vessels (brown arrows) of the retina in dog L3.

Figure 2-5: Pure rod (A), pure cone (B) and mixed rod/cone (C) ERG response amplitudes and b-wave:a-wave amplitude ratios for the mixed rod/cone response (D) for dog E1. Black and gray traces represent historical data \pm standard error of the mean (SEM) from normal $(n=7)$ and CLN2-affected $(n=6)$ untreated dogs, respectively, from the same research colony.

Figure 2-6: Mean $\pm S E M(A)$ and individual $(B-D)$ ERG rod response amplitudes for dogs E2 (B), E3 (C), and E4 (D). Mean rod response b-wave amplitudes in the rhTPP1-treated eyes (OS, blue traces) were greater than in the contralateral vehicle-treated eyes $\left(\mathrm{OD}\right.$, red traces) $\left({ }^{*} \mathrm{p}<0.05\right)$. Black and gray traces represent 
historical data \pm SEM from normal $(n=7)$ and CLN2-affected $(n=6)$ untreated dogs, respectively, from the same research colony.

Figure 2-7: Mean \pm SEM $(A)$ and individual (B-D) ERG cone response amplitudes for dogs E2 (B), E3 (C), and E4 (D). Cone response b-wave amplitudes in the rhTPP1-treated eye (OS, blue traces) were greater than the contralateral vehicletreated eye (OD, red traces) $(\uparrow p<0.01, \pm p<0.001)$. Black and gray traces represent historical data \pm SEM from normal $(n=7)$ and CLN2-affected $(n=6)$ untreated dogs, respectively, from the same research colony. 60

Figure 2-8: Mean \pm SEM (A) and individual (B-D) ERG mixed rod/cone response amplitudes for dogs E2 (B), E3 (C), and E4 (D). Mean mixed response b-wave amplitudes in the rhTPP1-treated eye (OS, blue traces) were greater than the contralateral vehicle-treated eye (OD, red traces) $\left({ }^{*} p<0.05, \dagger p<0.01, \pm p<0.001\right)$. Black and gray traces represent historical data \pm SEM from normal $(n=7)$ and CLN2-affected $(n=6)$ untreated dogs, respectively, from the same research colony.

Figure 2-9: ERG waveforms from dog E2 at 3, 6, and 9 months of age.

Responses from the rhTPP1-treated eye (OS, blue traces) remained normal or greater than normal throughout the study period, while the response from the vehicle control eye (OD, red traces) declined dramatically with age, as is typical for untreated CLN2-affected dogs.

Figure 2-10: Individual rod response amplitudes from dogs L1, L2, L3 and L4. Post symptomatic intravitreal rhTPP1 halted decline of retinal rod responses in CLN2-affected dogs. Intravitreal injections \#1 and \#2 were given within 1 week following the Baseline \#1 and \#2 ERG measurements, respectively. Black and gray traces represent historical data \pm SEM from normal $(n=7)$ and CLN2affected $(n=6)$ untreated dogs, respectively, from the same research colony..... 64

Figure 2-11: Individual cone response amplitudes from dogs L1, L2, L3 and L4. Post symptomatic intravitreal rhTPP1 halted decline of retinal cone responses in CLN2-affected dogs. Black and gray traces represent historical data \pm SEM from normal $(n=7)$ and CLN2-affected $(n=6)$ untreated dogs, respectively, from the same research colony. 65

Figure 2-12: Individual mixed rod/cone responses in late started dogs L1, L2, L3 and L4. Post symptomatic intravitreal rhTPP1 halted decline of retinal mixed rod and cone responses in CLN2-affected dogs. Black and gray traces represent historical data \pm SEM from normal $(n=7)$ and CLN2-affected $(n=6)$ untreated dogs, respectively, from the same research colony.

Figure 2-13: ERG waveforms from Dog L1 at 5, 8, and 10 months of age. (A) pure rod, (B) mixed rod and cone, (C) pure cone, and (D) $30 \mathrm{~Hz}$ flicker responses all exhibited ERG preservation in the rhTPP1-treated eye relative to the control eye. 
Figure 2-14: Quantification of nuclei in the inner nuclear layer from several retinal areas (central, superior mid-peripheral and inferior mid-peripheral retina) of dogs treated pre-symptomatically $(A, n=4)$ and post-symptomatically $(B, n=4)$ + SEM, ${ }^{*} p<0.05$.

Figure 2-15: Quantification of nuclei in the outer nuclear layer from several retinal areas (central, superior mid-peripheral and inferior mid-peripheral retina) of dogs treated pre-symptomatically $(A, n=4)$ and post-symptomatically $(B, n=4)$ + SEM, ${ }^{*} \mathrm{p}<0.05$.

Figure 2-16: Representative light micrographs of sections from the central posterior retinas of the rhTPP1-treated (A) and vehicle-treated (B) eyes of dog $\mathrm{E} 2$, euthanized at end-stage disease at approximately 11 months of age. Bar in (B) indicates the magnification of both micrographs.

Figure 2-17: Representative light micrographs of sections from the central retinas of the vehicle-treated $(A)$ and rhTPP1-treated $(B)$ eyes of dog L3, euthanized at end-stage disease at approximately 10 months of age. Bar in (A) indicates the magnification of both micrographs.

Figure 2-18: Electron micrographs of the base $(A)$ and the apical end $(B)$ of rod outer segments from the rhTPP1-treated eye and of the base $(C)$ and apical end (D) of from the vehicle-treated eye of dog E2. Normal morphology was retained at the bases of the rod outer segments in both rhTPP1- and vehicle-treated eyes (A \& C). However, while the disc membranes from the rhTPP1-treated eyes remained tightly packed, straight and parallel to one another at the distal ends of the rod outer segments, they were more loosely packed and less straight in the vehicle-treated eyes. The dog was euthanized at approximately 11 months of age. Bar in (A) indicates the magnification of all four micrographs.

Figure 2-19: Electron micrographs of the photoreceptor-retinal pigment epithelium interfaces from the vehicle-treated (A and B) and rhTPP1-treated (C) eyes of dog L4, euthanized at approximately 10 months of age. In the rhTPP1treated eyes, the ultrastructure of the rod outer segments was relatively normal, and the distal ends of the outer segments were closely apposed to the apical side of the retinal pigment epithelium (RPE) (C). In contrast, in the vehicletreated eyes only a fraction of the outer segments extended to the RPE apical surface and the apical ends of those that did exhibited an abnormal arrangement of the disc membranes $(A)$. Those that did not exhibited an abnormal irregularity in disc electron density and were unsheathed by long thin RPE apical processes. Rod outer segments (os); retinal pigment epithelium (rpe); rpe apical microvilli (arrows). Bar in each micrograph indicates the magnification.

Figure 2-20: Electron micrographs of the retinal outer plexiform layer (opl) and adjacent portions of the inner nuclear layer (inl) and outer nuclear layer (onl) from the rhTPP1-treated $(A)$ and vehicle-treated $(B)$ eyes of dog E2 euthanized at 
approximately 11 months of age. Axons from inl cells are indicated by arrow. Bar in each micrograph indicates magnification.

Figure 2-21: Electron micrographs of the outer plexiform layer/outer nuclear layer interface of the vehicle-treated (A) and rhTPP1-treated (B) eyes from dog L4. Rod photoreceptor nuclei (n); nerve fibers of bipolar cells (nf). Bar in (B) indicates the magnification of both micrographs.

Figure 2-22: Light micrographs of the ciliary bodies (cb) and adjacent portions of the scleras (s) from the rhTPP1-treated (A) and vehicle-treated (B) eyes of dog E2 euthanized at approximately 11 months of age. Bar in each micrograph indicates the magnification. 76

Figure 2-23: Light micrographs of the ciliary bodies from the vehicle-treated $(A)$ and rhTPP1-treated (B) eyes of dog L3 euthanized at approximately 10.5 months of age. Bar in $(A)$ indicates the magnification of both micrographs. 76

Figure 2-24: Electron micrographs of areas of the ciliary bodies from the rhTPP1-treated (A) and vehicle-treated (B) eyes of dog E2 illustrating the tight packing between the cells and extracellular matrix in the treated eye and the presence of large gaps within the tissue of the vehicle-treated eye associated with a decrease in collagen (c) density, and a thinning of the stromal cell bodies (s). melanocytes $(\mathrm{m})$. Bar in both micrographs indicates the magnification........ 77

Figure 2-25: Merged light microscopic image of an optic nerve cross-section from the rhTPP1-treated eye of dog L1 (A) and an enlarged area of this image showing counting of individual axons (B)

Figure 2-26: Electron micrographs of retinal ganglion cells from the vehicletreated eye $(\mathrm{A})$ and the rhTPP1-treated eye (B) from dog L4. Ganglion storage bodies "SB" and arrows, nerve fiber layer "NFL". Bar in each micrograph indicates the magnification.

Figure 3-1: A fluorescence SLO/OCT image of material in the GFP-MSC eye (A) and an SLO/OCT image of material in the TPP1-MSC eye (B) of dog 9 at 8 months of age. A fluorescence stereoscope image of the vitreous from the GFPMSC eye of this dog who was euthanized at 10.5 months of age (C). ........... 106

Figure 3-2: Mean mixed rod and cone (A), pure rod (B), pure cone (C) and cone flicker (D) b-wave amplitudes from dogs that received intravitreal TPP1-MSCs (blue line, $\pm S E M, n=6$ ) in one eye and GFP-MSCs (red line, $\pm S E M, n=6$ ) in the contralateral eye. Black and gray traces represent historical data from normal $(n=7)$ and CLN2-affected $(n=6)$ untreated dogs from the same research colony $( \pm$ SEM). $\ddagger p \leq 0.001, \dagger p=0.003$

Figure 3-3: Mixed rod and cone (A), pure rod (B), pure cone (C) and cone flicker (D) b-wave amplitudes from dog 6 that received intravitreal TPP1-MSCs in one eye and GFP-MSCs in the contralateral eye. 108 
Figure 3-4: Kaplan-Meier Curve showing survival probability of untreated affected $(n=6)$ dogs and dogs who received CSF infusions of TPP1-MSCs $(n=6)$ and mCherry-MSCs $(n=2)$.

Figure 3-5: Onset of neurologic disease signs + standard deviation in untreated dogs $(n=11)$ and in dogs that received CSF infusions of TPP1-MSCs $(n=6)$ and mCherry-MSCs $(n=2)$. No MSC-treated dogs developed pathologic nystagmus. $\mathrm{PL}=$ pelvic limbs; $\mathrm{TL}=$ thoracic limbs; $\mathrm{AL}=$ all limbs

Figure 3-6: Total incorrect choices (A) and change in total incorrect choices from 4 months of age $(C)$ for monthly T-maze assessment for unaffected (black line, $n=17, \pm S E M$ ), untreated CLN2-affected (grey line, $n=17, \pm S E M$ ) and TPP1-MSC treated (blue line, $n=6, \pm S E M$ ) dogs. Individual total incorrect choices of TPP1MSC treated dogs (B) and change in total incorrect choices (D) are also shown.

Figure 3-7: Ventricular volume over time in unaffected (black line, $n=5, \pm S E M)$, untreated CLN2-affected (grey line, $n=8, \pm S E M$ ) and TPP1-MSC treated (blue line, $n=6, \pm S E M)$ dogs.

Figure 3-8: MR images from dog 9, who received a CSF infusion of TPP1MSCs, at 3.5 and 9.5 months of age. Enlarged ventricles and reduced cortical grey matter are apparent at 9.5 months of age.

Figure 4-1: Diagram showing DNA constructs used in this study. ITR = AAV2 inverted terminal repeat; WPRE $=$ woodchuck hepatitis virus posttranscriptional regulatory element; bGH poly $(A)=$ bovine growth hormone polyadenylation signal; $6 \mathrm{xHis}=$ polyhistidine tag

Figure 4-2: Fluorescence SLO images of dogs $A(A), B(B), C(C)$ and $G(D)$ showing dose-dependent transduction of the retina with AAV2.CAG.GFP. Doses of $6 \times 10^{9}(\mathrm{~A})$ and $6 \times 10^{10} \mathrm{vg}(\mathrm{B})$ resulted in only modest GFP expression. $6 \times 10^{11}$ vg (C) resulted in widespread GFP expression but did not preserve ERG amplitudes. Only the highest dose tested, $1 \times 10^{12} \mathrm{vg}$, both resulted in widespread GFP expression (D) and preserved retinal function.

Figure 4-3: Fluorescence SLO images of the GFP-control retina of dog $E$ at 0 (A), 2 (B), 4 (C) and 8 (D) weeks post-treatment showing increasing fluorescent signal over the first 8 weeks after treatment.

Figure 4-4: Low magnification fluorescence stereoscope image of the central superior retina from the GFP-control retina of Dog $G(A)$ and a fluorescence micrograph of a section from the same region $(B)$. onh $=$ optic nerve head; $v=$ vitreous; ilm = inner limiting membrane; $\mathrm{gcl}=$ ganglion cell layer; $\mathrm{ipl}=\mathrm{inner}$ plexiform layer; inl = inner nuclear layer; onl = outer nuclear layer; olm = outer limiting membrane; $r p e=$ retinal pigment epithelium 
Figure 4-5: Micrographs of TPP1-stained retinas from the superior midperipheral region of TPP1-treated (A) and GFP-control (B) eyes of dog G. The arrow shows a labeled RPE cell. $\mathrm{v}=$ vitreous; $\mathrm{gcl}=$ ganglion cell layer; $\mathrm{ipl}=$ inner plexiform layer; inl = inner nuclear layer; onl = outer nuclear layer; os = photoreceptor outer segments; rpe = retinal pigment epithelium

Figure 4-6: SLO fundus images and corresponding OCT images from the center of the optic nerve head from dogs treated with the therapeutic dose of AAV2.CAG.hTPP1. Fluorescence SLO images from the GFP-control eye are shown at the end of each row. Yellow arrows indicate deposits of intravitreal triamcinolone.

Figure 4-7: Mixed rod and cone (A), pure rod (B), pure cone (C) and cone flicker (D) response amplitudes from dog $\mathrm{D}$. Black and gray traces represent historical data \pm SEM from normal $(n=7)$ and CLN2-affected $(n=6)$ untreated dogs, respectively, from the same research colony.

Figure 4-8: Mixed rod and cone (A), pure rod (B), pure cone (C) and cone flicker (D) response amplitudes from dog $\mathrm{E}$. Black and gray traces represent historical data \pm SEM from normal $(n=7)$ and CLN2-affected $(n=6)$ untreated dogs, respectively, from the same research colony.

Figure 4-9: ERG waveforms from dog $E$ at 4, 7 and 10 months of age. Responses from the AAV2.CAG.hTPP1 treated eye (blue traces) remained normal or greater than normal throughout the study period, while the responses from the AAV2.CAG.GFP control eye (red traces) declined as expected with age.

Figure 4-10: Mixed rod and cone (A), pure rod (B), pure cone $(C)$ and cone flicker (D) response amplitudes from dog F. Black and gray traces represent historical data \pm SEM from normal $(n=7)$ and CLN2-affected $(n=6)$ untreated dogs, respectively, from the same research colony.....

Figure 4-11: Mixed rod and cone (A), pure rod (B), pure cone $(C)$ and cone flicker (D) response amplitudes from dog $\mathrm{G}$. Black and gray traces represent historical data \pm SEM from normal $(n=7)$ and CLN2-affected $(n=6)$ untreated dogs, respectively, from the same research colony

Figure 4-12: Mixed rod and cone (A), pure rod (B), pure cone $(C)$ and cone flicker (D) response amplitudes from dog $\mathrm{H}$. Black and gray traces represent historical data \pm SEM from normal $(n=7)$ and CLN2-affected $(n=6)$ untreated dogs, respectively, from the same research colony.

Figure 4-13: Mixed rod and cone (A), pure rod (B), pure cone $(C)$ and cone flicker (D) response amplitudes from dog I. Black and gray traces represent historical data \pm SEM from normal $(n=7)$ and CLN2-affected $(n=6)$ untreated dogs, respectively, from the same research colony. 
Figure 4-14: Average mixed rod and cone (A), pure rod (B), pure cone $(C)$ and cone flicker (D) b-wave amplitudes \pm SEM from dogs $E$-I. $\ddagger p \leq 0.001, \dagger p<0.01$. Black and gray traces represent historical data \pm SEM from normal $(n=7)$ and CLN2-affected $(n=6)$ untreated dogs, respectively, from the same research colony.

Figure 5-1: Elements of the VEP indicating the three positive peaks (P1, P2, and $\mathrm{P} 3$ ) and the two negative peaks (N1 and N2) that are typically present in normal healthy dogs when the recordings are performed as described for the present study (Strain et al., 1990). 162

Figure 5-2: Diagram illustrating the eye dissections. N:nasal; T:temporal; S:superior; I:inferior. Black circle: optic nerve head. The eyecups were dissected into the regions indicated by $A-G$. For this study, microscopic analyses were performed on the region of the retina indicated by the shaded rectangle. 165

Figure 5-3: SLO and OCT images of $\operatorname{dogs} X(A), Y(B)$ and $Z(C)$ at 7 months of age and dogs $X(D), Y(E), W(F)$ and $Z(G)$ at 23 months of age. 1-3 small bullous retinal detachment lesions were present in both eyes of dog $W(F)$ and $\operatorname{dog} Z(G)$ by 23 months of age. Green lines indicate the locations from which the OCT images were obtained. Arrows indicate the locations of the retinal lesions.

Figure 5-4: Mean ERG response amplitudes $\pm S E M$ for all 5 dogs. Cone function was primarily affected. Mean cone $(\# p<0.01, E)$, cone flicker $\left({ }^{*} \mathrm{p}<0.05, \mathrm{H}\right)$ and mixed rod and cone $(\# p<0.01, B) b$-wave amplitudes were significantly reduced from baseline at 23 months of age. The mean cone a-wave amplitude ( $F)$ was significantly decreased at 23 months of age $\left({ }^{*} p<0.05\right)$ while the mixed rod and cone a-wave $(C)$ was unchanged. Mean cone $\left({ }^{*} p<0.05, G\right)$ and mixed rod and cone $(\# p<0.01, D)$ b:a wave ratios were significantly decreased from baseline at 23 months of age. Mean rod response (A) was not significantly changed from baseline at 23 months of age ..................................................................... 170

Figure 5-5: ERG waveforms from dog $X$ at 7,16 and 23 months of age. ....... 171

Figure 5-6: Mean VEP P2 amplitudes \pm SEM for all 5 dogs (A) and the mean ratio of the P2 amplitude to the cone b-wave amplitude \pm SEM for all 5 dogs (B). The mean amplitude of P2 was decreased by 23 months of age ( $\left.{ }^{*} \mathrm{p}<0.05\right)$. The mean ratio of the $\mathrm{P} 2$ amplitude to the cone $b$-wave amplitude was increased by 23 months $\left({ }^{*} \mathrm{p}<0.05\right)$.

Figure 5-7: VEP waveforms from $\operatorname{dog} Y(A)$ and $\operatorname{dog} Z(B)$ at 7, 16 and 23 months of age. Arrows indicate P2. P2 was identified as the first positive peak following N1, following the standard convention. In some of the recordings, there was an additional positive peak following P1 (asterisks) that has not been reported in normal dogs. 
Figure 5-8: Light micrograph of a section of the superior-central retina from dog Y, euthanized at 23.4 months of age. All layers of the retina were well preserved with no apparent morphological abnormalities.

Figure 5-9: Fluorescence micrographs of the inner (A) and outer $(B)$ layers of unstained cryostat sections of the retina from $\operatorname{dog} Z$, euthanized at 23.4 months of age. Bar in (B) indicates the magnification of both micrographs.

Figure 5-10: Fluorescence micrographs of unstained cryostat sections of the occipital cortex from dog $\mathrm{V}(\mathrm{A})$ and $\operatorname{dog} \mathrm{Y}(\mathrm{B})$, euthanized at 20.1 and 23.4 months of age respectively. Bar in (B) indicates the magnification of both micrographs.

Figure 5-11: Light micrographs of sections of the retina (A) and occipital cortex (B) from dog X. Sections were immunostained for LAMP2 localization. No counterstain was used. In the retina, LAMP2 immunostaining was most pronounced in the ganglion cells, along the outer limiting membrane, and in the RPE. In the occipital cortex most cells contained aggregates of LAMP2-positive inclusions. Bar in (B) indicates the magnification of both micrographs.

Figure 5-12: Electron micrograph of a neuron in the occipital cortex of an affected Golden Retriever (dog V) euthanized at approximately 20 months of age.

Figure 5-13: Electron micrographs of disease-specific storage bodies in retinal ganglion cells of an affected Golden Retriever (dog V) euthanized at approximately 20 months of age. In certain planes of section some of the contents of the storage bodies had a paracrystalline appearance.

Figure 5-14: Electron micrograph showing an accumulation of disease-specific storage bodies (s) in a photoreceptor cell of an affected Golden Retriever (dog V) euthanized at approximately 20 months of age. The storage bodies were present almost exclusively just interior to the retinal outer limiting membrane (arrows) and exterior to the photoreceptor nuclei (n). 180

Figure 5-15: Electron micrographs of disease-specific storage bodies in the retinal pigment epithelium of an affected Golden Retriever (dog W) euthanized at approximately 22 months of age. The ultrastructural appearances of the storage bodies (arrows in A and inclusions in B and $\mathrm{C}$ ) were quite heterogenous. Some inclusions contained melanosomes $(\mathrm{m})$ and a small minority appeared to be phagocytosed photoreceptor outer segments $(p)$. The contents of some inclusions had a membrane-like appearance $(B)$. Some inclusions were surrounded by a double membrane characteristic of autophagosomes (arrows in C)

Figure 5-16: Electron micrographs of additional disease-specific storage bodies in the retinal pigment epithelium of an affected Golden Retriever (dog W) 
euthanized at approximately 22 months of age. The ultrastructural appearances of the storage bodies were quite heterogeneous, even within the same eye. .. 182

Figure 5-17: Light micrograph of a cross-section of the optic nerve from $\operatorname{dog} Y$ stained with Masson's trichrome. The blue-staining connective tissue septa (arrows) only partially surround axon fascicles. ........................................... 183 


\section{LIST OF TABLES}

Table 2-1: Pre-symptomatic enzyme replacement therapy dosing schedule. Grey coloring indicates length of interval between doses.

Table 2-2: Post-symptomatic enzyme replacement therapy dosing schedule ... 35

Table 2-3: Concentrations of rhTPP1 in the aqueous and vitreous of rhTPP1-

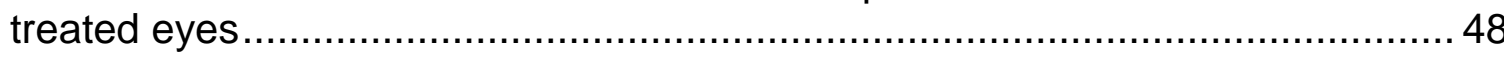

Table 2-4: Perivascular cuffing around inner retina vessels ............................55

Table 2-5: Anti-TPP1 antibody titers in pre-euthanasia blood samples ............. 56

Table 2-6: Numbers of axons in the optic nerves of late start dogs .................. 78

Table 2-7: Effect of rhTPP1 treatment on storage body content of retinal ganglion cells

Table 3-1: Treatment details for MSC injections. Details for IVT injections of TPP1-MSCs in the left eyes are shown. Contralateral eyes received GFP-MSCs of the same passage number, and additional details for control eyes including number of cells injected can be found in Appendix A. AAV vectors were used to deliver the transgene. In some cases, a portion of TPP1-MSCs were transduced to also express a fluorescent marker (i.e., 25\% TPP1 \& GFP and 75\% TPP1 only). IVT = intravitreal, $\mathrm{CM}=$ injection at cisterna magna, ICV = intracerebroventricular injection.

Table 4-1: Intravitreal gene therapy treatments; $v g$ = viral genomes

Table 4-2: Summary of anti-inflammatory treatments. $w=$ weeks; $d / c=$ discontinued *Cyclosporine and leflunomide were initiated 2 weeks before treatment. ${ }^{* *}$ All dogs received sub-Tenon's triamcinolone immediately following vector injection.

Table 5-1: Ages at which dogs were euthanized ........................................ 163

Table 5-2: Cotton ball visual tracking assessments ..................................... 173 


\title{
DISEASE CHARACTERIZATION AND PRE-CLINICAL TESTING IN CANINE MODELS OF THE NEURONAL CEROID LIPOFUSCINOSES
}

\author{
Grace Robinson Kick \\ Dr. Martin L. Katz, Dissertation Supervisor
}

\begin{abstract}
The neuronal ceroid lipofuscinoses (NCLs) are inherited lysosomal storage disorders characterized by intracellular accumulation of autofluorescent storage material and by progressive degeneration of neurons in the brain and retina. Clinically, the NCLs are characterized by progressive cognitive and motor impairment, seizures and progressive loss of vision culminating in premature death. Variants in at least 14 genes are known to cause forms of NCL. The CLN2 form of NCL is caused by variants in the TPP1 gene, which encodes the soluble lysosomal hydrolase tripeptidyl peptidase 1 (TPP1), while the CLN5 form is caused by variants in CLN5, which encodes a lysosomal protein the function of which has not been definitively determined.
\end{abstract}

CLN2 disease has a late-infantile onset and typically culminates in death in the early teenage years. A canine disease model of affected Dachshunds with CLN2 disease recapitulates the features of the human disease and has proven to be an excellent model for preclinical therapy testing. CLN2-affected Dachshunds are homozygous for a single base pair deletion in the canine ortholog of TPP1 which results in a premature termination codon and lack of functional TPP1 protein. Affected dogs present with progressive motor, cognitive and behavioral 
changes, myoclonic seizures and brain atrophy which progress to end-stage disease requiring humane euthanasia around 10 months of age. Affected dogs also exhibit vison loss associated with progressive retinal degeneration, electroretinogram (ERG) deficits and degeneration of visual centers in the brain.

Previous work in the CLN2 Dachshund model demonstrated that delivering functional TPP1 protein to the central nervous system via enzyme replacement therapy (ERT) or gene therapy extends lifespan and delays neurological disease progression. However, these treatments did not preserve retinal structure or function. We therefore conducted studies to assess three different methods of delivering functional TPP1 to the retina: (1) intravitreal enzyme replacement therapy, (2) ex vivo cell-mediated gene therapy and (3) in vivo intravitreal gene therapy. Additionally, we tested an ex vivo cell-mediated gene therapy approach to deliver TPP1 to the central nervous system as an alternative to the ERT and gene therapy methods previously assessed in the canine model.

Intravitreal ERT was tested both before and after onset of declines in ERG amplitudes. ERT was able to fully preserve ERG b-wave amplitudes when administered pre-symptomatically. Post-symptomatic treatment halted progression of disease-related declines in amplitudes through end stage neurological disease at approximately 10 months of age. The major side effect of treatment was transient intraocular inflammation characterized by anterior and posterior uveitis and by the production of anti-TPP1 antibodies. Treatment also preserved retinal structure, prevented disease-related loss cells in the inner and outer nuclear layers and reduced the accumulation of intracellular storage 
material in retinal ganglion cells. These results were incorporated into an application that is being submitted to the Food and Drug Administration and European regulatory agencies for approval to conduct a clinical trial of this treatment to prevent progressive vision loss in children with CLN2 disease.

A single intravitreal administration of TPP1-overexpressing autologous mesenchymal stem cells (TPP1-MSCs) was able to partially preserve retinal function. ERG rod b-wave amplitudes remained larger in treated eyes than in control eyes through 10 months of age, although amplitudes continued to decline in treated eyes. TPP1-MSCs were safe and well-tolerated and did not cause inflammation. While these results are encouraging, further studies are necessary to optimize this therapy.

One-time intravitreal administration of a gene therapy vector (AAV2.CAG. TPP1) prior to the onset of retinal function impairment partially preserved retinal function through end-stage neurological disease at approximately 10 months of age. The vector transduced primarily cells of the inner retina and resulted in transgene expression for at least 6 months after treatment. There was also transgene expression in the retinal pigment epithelium. As with intravitreal ERT, a side effect was intraocular inflammation characterized by anterior and posterior uveitis. Inflammation was only partially alleviated by treatment with various anti-inflammatory medications. These results support further investigation to develop this approach to preserve vision in CLN2 disease. 
To date, infusion of TPP1-expressing mesenchymal stem cells into the cerebrospinal fluid has not been effective in treating central nervous system pathology in CLN2 disease. So far, with two minor exceptions, this method has not extended lifespan, slowed neurological disease progression or impeded brain atrophy. These exceptions, a slightly increased lifespan of one dog and temporarily improved performance on a cognitive test by another, may be indicative of some therapeutic benefit in those dogs. Work is ongoing to determine why this approach has not been successful and to modify the approach to enhance the potential for efficacy. This current work suggests that reducing the in vitro expansion of MSCs and/or removing immunosuppressive medications from the protocol may have improved efficacy, since these modifications were implemented for the dogs that demonstrated slight increases in lifespan and cognitive function. However, even in these two dogs any improvements were minor. Lack of success could be due to failure of the MSCs to survive long-term in the CNS or to secrete therapeutic amounts of hTPP1. Future studies could include alternative delivery methods and repeated injections over the disease course. Going forward, efforts should focus on determining if MSCs survive in the CNS, and if so for how long, as well as whether therapeutic concentrations of hTPP1 are obtainable with this method.

The CLN5 form of NCL is characterized by progressive neurological decline and vision loss. As with CLN2 disease, a CLN5 canine model disease would be a valuable resource for testing potential therapeutic interventions. We characterized visual system pathology in five Golden Retrievers homozygous for 
a two base pair deletion and frameshift in the canine ortholog of CLN5. The dogs exhibited visual impairment by 22 months of age, progressive decline in ERG amplitudes primarily affecting cones and altered visual evoked potential recordings indicating impaired visual signal processing in the brain. While there were no gross retinal abnormalities by 23 months of age, there was extensive storage material accumulation in the retina and occipital cortex by 20 months of age. The visual system pathology in these dogs was similar to that seen in human patients. The baseline data obtained from the untreated affected dogs with CLN5 disease will serve as a foundation for designing therapeutic intervention studies. 


\section{Introduction \& Background}

\subsection{The Neuronal Ceroid Lipofuscinoses}

\subsubsection{Neuronal Ceroid Lipofuscinoses in Humans}

The neuronal ceroid lipofuscinoses (NCLs) were first recognized as a group of familial diseases characterized by loss of vision, cognitive and motor decline and intraneuronal storage with varying ages of onset and rates of progression. The term 'neuronal ceroid lipofuscinosis' (NCL) was adopted in reference to their characteristic neuronal accumulation of lipofuscin-like autofluorescent lysosomal storage material (Katz and Robison, 2002; Seehafer and Pearce, 2006). The NCLs are predominately inherited in an autosomal recessive manner and usually exhibit childhood onset. Their combined incidence is estimated at 1.3-13.6 per 100,000 live births (Williams, 2011). Common signs of the NCLs include seizures, visual deficits and blindness, dementia, motor impairment, cognitive decline, and psychiatric symptoms. They are typically fatal, especially in cases of childhood onset, from complications associated with swallowing and breathing.

Variants in 14 genes have been associated with different forms of NCL (Warrier et al., 2013). The proteins encoded by these genes include soluble lysosomal enzymes, lysosomal membrane proteins, endoplasmic reticulum membrane proteins and vesicle-associated proteins (Kollmann et al., 2013). 
The defining histopathological features of the NCLs are accumulation of autofluorescent storage bodies in most tissues and progressive and selective neuronal loss in the cortex, cerebellum and retina (Haltia and Goebel, 2013). The storage material does not consist primarily of a metabolic intermediate as in many other lysosomal storage diseases; it is instead a heterogeneous mixture of protein, lipid and carbohydrate. Nevertheless, in certain NCL subtypes the primary protein component is either sphingolipid activator proteins (SAPs) A \& D or subunit C of mitochondrial ATP synthase (SCMAS) (Anderson et al., 2013). In general, storage material consisting primarily of subunit $\mathrm{C}$ of mitochondrial ATP synthase exhibits membrane-like curvilinear, rectilinear and fingerprint patterns whereas storage material primarily composed of SAPs forms membrane-bound granular osmiophilic deposits. While storage material accumulation does not correlate well with neuronal loss, it is readily observed in extracerebral tissues and has storage bodies that are characteristic of different subtypes. Although the storage material is present in most tissues of affected individuals, neurons of the brain and retina are uniquely affected, and degeneration of these cells is responsible for neurologic and visual deficits.

Medical management of the NCLs has long been limited to palliative, supportive and symptomatic care, such as anticonvulsants to treat seizures, physical therapy, educational support and alternate feeding methods. Therefore, developing disease-modifying therapies for the NCLs is an important goal. 


\subsubsection{Neuronal Ceroid Lipofuscinosis Type 2 (CLN2 Disease)}

CLN2 Disease is caused by deleterious variants in TPP1 that result in decreased or abolished enzymatic activity of the gene product, the lysosomal protease tripeptidyl peptidase 1 (TPP1) (Sleat et al., 1997; Vines and Warburton, 1999). TPP1 cleaves tripeptides from the $\mathrm{N}$ terminus of small polypeptides in the lysosome with maximal activity at pH 4.5 (Ezaki et al., 2000; Vines and Warburton, 1998). In vitro, TPP1 has been shown to cleave several peptide hormones as well as subunit C of mitochondrial ATP synthase, but its natural in vivo substrates are unknown (Ezaki et al., 1999; Kollmann et al., 2013).

TPP1 is initially synthesized as a catalytically inactive $66 \mathrm{kDa}$ pro-enzyme decorated with high-mannose type glycans at its five $\mathrm{N}$-glycosylation sites (Golabek et al., 2003; Lin et al., 2001). Glycosylation of TPP1 plays important roles in folding, processing and trafficking of the enzyme (Wujek et al., 2004). The mannose-6-phosphate (M6P) pathway is the major mechanism by which lysosomal hydrolases are trafficked to the lysosome. In the Golgi apparatus, the TPP1 pro-enzyme acquires a M6P tag on high-mannose type glycans which can be bound by a mannose-6-phosphate receptor (M6PR). M6PRs bind M6Ptagged TPP1 in the Golgi and the complex is trafficked via clathrin-coated vesicles to endosomes. The M6PR and TPP1 dissociate as the $\mathrm{pH}$ drops in the developing late endosome (Braulke and Bonifacino, 2009; Coutinho et al., 2012). The compartment further acidifies as the endosome matures, and in the acidic lysosomal lumen the TPP1 zymogen self-activates by auto-catalytic processing to the $46 \mathrm{kDa}$ enzymatically active form (Lin et al., 2001). However, a fraction of 
the newly synthesized TPP1 pro-enzyme is not bound by M6PR and instead enters the default secretory pathway. After secretion into the extracellular space, the enzyme can be bound by M6PRs on the plasma membrane of the secreting cell or of neighboring cells and delivered to the lysosome (Braulke and Bonifacino, 2009; Coutinho et al., 2012).

TPP1 is expressed in most tissues and cell types, both neural and extraneural, at varying levels of expression. There does not appear to be any correlation between TPP1 expression and the degeneration of specific cell types in CLN2 disease (Palmer et al., 2013). Despite the ubiquitous expression of TPP1 and the presence of storage material in both neural and extraneural tissues, degeneration occurs primarily in neurons of the brain and retina (Anderson et al., 2013). However, disease-related pathology does occur systemically (Anderson et al., 2013; Katz et al., 2017a; Rietdorf et al., 2020). Even within the brain there is not a relationship between TPP1 expression and the degeneration of specific subsets of neurons (Kida et al., 2001).

More than 100 disease-causing variants in TPP1 have been identified, including small indels, missense, nonsense and splice site variants ("NCL Mutation and Patient Database," n.d.). Some of these variants result in undetectable TPP1 enzymatic activity in individuals with mutations in both copies of TPP1 through loss of enzymatic activity or complete lack of protein production. The two most common CLN2 mutations are a splice site mutation and a nonsense mutation (Gardner et al., 2019; Kousi et al., 2012; Mole, 2004) 
Although the pathological mechanism is not understood, lack of TPP1 catalytic activity leads to intracellular accumulation of ceroid lipofuscin-like lipopigment and degeneration of neurons in the brain and retina. Lipopigment accumulates in most tissues, but in extracerebral tissues this may be the only observable histopathological change (Anderson et al., 2013; Radke et al., 2015). Classic CLN2 storage bodies contain abundant SCMAS and are of the curvilinear profile type: curved, thin lamellae surrounded by a single membrane (Anderson et al., 2013).

The brains of late stage CLN2 patients are severely atrophied with corresponding enlargement of the fourth ventricle in particular as well as the cisterna magna and lateral ventricles (Chang et al., 2011; Dunn, 1987). Brain atrophy and ventricular enlargement measured by magnetic resonance imaging (MRI) correlate well with clinical disease progression (Löbel et al., 2016). At end stage disease, the cerebrum, cerebellum, thalamus and hippocampal formation are all severely atrophied as a result of neuronal death (Autti et al., 1997; Kida et al., 2001; Tyynelä et al., 2004). Reduction of white matter is apparent as well, though the changes are milder than those seen in grey matter (Autti et al., 1997; Petersen et al., 1996).

Vision loss in CLN2 patients likely results from both degeneration of the retina and of the visual processing areas of the brain. Retinas of affected children display profound degeneration of several retinal cell types, particularly photoreceptors (Goebel et al., 1977; Orlin et al., 2013; Schochet et al., 1980). Retinal function can be assessed with full-field electroretinogram (ERG) 
recordings, which measure the summed electrical response of the retina in response to a light stimulus. The ERG waveform can be analyzed to obtain information about the health of specific layers of the retina. Of most use clinically are the a-wave and b-wave. The negative a-wave reflects the hyperpolarization of photoreceptors and the positive b-wave arises from cells of the inner nuclear layer post-synaptic to photoreceptors, especially depolarization of ON-center bipolar cells. ERG amplitudes in CLN2-affected children decrease over time and are ultimately abolished (Chang et al., 2011; Weleber, 1998). These recordings show an early and severe reduction of b-wave amplitudes indicating early loss of inner retinal function. A later and milder reduction of a-wave amplitudes reflects onset of photoreceptor dysfunction, with the cone response initially and more severely reduced and loss of the rod response occurring latest in the disease course. Additionally, the pupils become less reactive over the course of disease (Weleber, 1998).

Children affected with CLN2 disease typically develop normally for the first 2-4 years of life then experience progressive psychomotor decline and regression ultimately leading to premature death between 8-12 years of age. The initial symptom that brings children to medical attention is typically seizures around 3 years of age, though language delay usually occurs first. Motor dysfunction manifests initially as decreased limb strength around age 4. Language impairment and motor dysfunction rapidly progress to complete loss of language and voluntary movement around 5-6 years of age. Motor dysfunction includes gait abnormalities, myoclonus, spasticity, dystonia and difficulty 
swallowing. Visual loss symptoms typically begin around age 4 and culminate in complete blindness around 7-10 years of age (Chang et al., 2011; Steinfeld et al., 2002; Worgall et al., 2007). Death typically occurs in adolescence as a result of impaired swallowing and respiration secondary to severe neurological impairment. A clinical scoring system, the Hamburg Scale, was developed to provide a quantitative measure of clinical disease progression. The scale rates progression as four categories: motor function, seizures, visual function and language (Steinfeld et al., 2002). CLN2 is a rapidly progressive disease; affected children progress from normal to no function on the motor and language subscales over approximately 30 months, and time to death after symptom onset is approximately 8 years (Nickel et al., 2018).

\subsubsection{The Canine Disease Model of CLN2 Disease}

Large animal models are useful in preclinical testing of potential therapeutic interventions. While initial work in rodents can be useful in early development of a therapeutic, these models are limited by their small size, primitive nervous systems and short lifespans. Studies in large animals with physiologies and anatomies more similar to those of humans can provide critical evidence regarding safety and efficacy that is more translatable to human patients and are an important intermediate in therapy development. Due to their larger size, large animal models allow for evaluation and treatment using similar equipment and techniques as would be used in humans as well as more accurate and translatable studies of biodistribution, pharmacodynamics and pharmacokinetics. Longer lifespans may also allow for long-term safety and 
efficacy studies. These models can also be used to evaluate potential biomarkers of disease progression that are translatable to human subjects (Gurda et al., 2017; Gurda and Vite, 2019; Story et al., 2020). Dogs are an excellent large animal model of human disease, with more than 200 human inherited diseases having natural disease-causing mutations in canine orthologs discovered (omia.org). To date, 12 mutations in the canine orthologs of 8 human NCL genes have been identified as causing canine NCL in several breeds (Katz et al., 2017b).

The lab identified a frameshift null mutation in the canine ortholog of TPP1 in miniature long-haired Dachshunds and established a research colony of CLN2-affected TPP1-/ dogs. The Dachshund disease recapitulates the major features of human CLN2 disease. Dogs homozygous for the TPP1 null mutation, a single nucleotide deletion in exon 4 (c.325delC) predicted to create a premature stop codon, have no detectable TPP1 activity. Tissues from affected dogs contain autofluorescent storage bodies with a curvilinear profile like those seen in the human disease (Awano et al., 2006). Clinical signs become apparent in these dogs around 6.5 months of age, around which time they begin to exhibit ataxia, myoclonic seizures, vision loss, and cognitive and behavioral changes including aggression and mental dullness. Seizures appear at approximately 7-8 months of age. The disease progresses rapidly, and affected dogs reach endstage between around 10 and 12 months of age.

Brain atrophy is apparent on MRI as thinning cortical grey matter, deepening sulci and enlargement of the ventricular system (Vuillemenot et al., 
2011). Early signs of cognitive changes can be detected by impaired performance on a reversal learning task in which the dog's ability to recall the location of a food reward is tested. Beginning as early as 4 months of age, affected dogs show impaired performance which worsens over time (Sanders et al., 2011, 2010). Motor symptoms include intention and resting tremors, cerebellar ataxia, myoclonic jerks and proprioceptive and postural reaction deficits and by 9 months of age affected dogs have great difficulty walking and eating (Katz et al., 2008; Sanders et al., 2011).

Deficits in retinal function as assessed with ERG recordings are apparent in CLN2-affected dogs with significant deficits appreciable by $3-5$ months of age and gradually declining further over time. As in human patients, the inner retina is affected early and most severely followed by more mild deficits in photoreceptors. Significant deficits in the scotopic rod b-wave amplitude can be detected beginning at 3 months of age and progress until the b-wave is abolished at 6-7 months of age. Photopic cone b-wave and scotopic mixed rod and cone amplitudes show a gradual reduction starting at 4 and 5 months of age, respectively. All b-wave responses are significantly reduced by 5 months of age. The scotopic and photopic a-wave, reflecting photoreceptor function, decline late in disease progression; deficits are not appreciable until 8-10 months of age. In dogs whose lifespan is extended with treatment, a- and b-wave amplitudes continue to decline, the b-wave is abolished and the ERG becomes nonrecordable under photopic and scotopic conditions by 12 months of age (Whiting et al., 2016). A reduced b:a-wave ratio indicates that the earliest and most severe 
site of degeneration is the inner nuclear layer. (Katz et al., 2008; Whiting et al., 2013).

CLN2-affected dogs are functionally blind by around 8 months of age; however, at this age ERG responses are still recordable, if severely diminished, suggesting that cortical blindness also contributes to vision loss. Later stage changes of the visual pathway can be detected by measurement of the pupillary light reflex (PLR) as pupil size is the result of balance between parasympathetic and sympathetic nervous system input of the autonomic nervous system. In response to changes in retinal illumination, the parasympathetic pathway decreases pupil diameter and the sympathetic pathway increases pupil diameter through pathways separate from the primary visual pathway (Liu, 2003). CLN2affected dogs have slower, reduced pupil constriction, smaller pupil size at baseline and slower redilation of the pupil, indicating impairment of both pathways. These deficits worsen as the disease progresses (Whiting et al., 2013).

At the time of necropsy, retinal degeneration is apparent morphologically as thinning of the retina and loss of photoreceptors and cells of the inner nuclear layer. By end-stage disease, storage material is present most retinal layers excluding the photoreceptor layer and retinal pigment epithelium (Katz et al., 2008). An additional feature of canine CLN2 disease that is not reported in human patients is the development of multifocal retinal detachments. These canine multifocal retinopathy $(\mathrm{CMR})$ lesions are areas where the neural retina has pulled away from the underlying retinal pigment epithelium (RPE). CMR 
lesions occur in many but not all affected dogs at variable ages and increase in number and size as the disease progresses (Whiting et al., 2015).

\subsubsection{Neuronal Ceroid Lipofuscinosis Type 5 (CLN5 Disease)}

CLN5 Disease is a variant form of late infantile NCL first recognized in Finland where the disease is especially prevalent (2.6 per million generally and even more concentrated in specific geographic areas) (Uvebrant and Hagberg, 1997). It rarely occurs outside of Finland. It is associated with sequence variants in CLN5 which encodes a lysosomal protein with no homology to any known protein and whose function remains unclear (Klockars et al., 1999a, 1999b; Savukoski et al., 1998). CLN5 is near ubiquitously expressed in adult human tissues (Basak et al., 2021; Savukoski et al., 1998). The subcellular localization and solubility of CLN5 have been extensively debated. It has been localized to the ER and lysosome, but it has been recognized that the mature wild type form is lysosomal (Isosomppi et al., 2002; Lebrun et al., 2009; Schmiedt et al., 2010; Vesa et al., 2002). Studies have argued that CLN5 is either membrane-bound or soluble, though the most recent evidence indicates that CLN5 is initially membrane-associated then cleaved into a soluble form (Basak et al., 2021; Bessa et al., 2006; Isosomppi et al., 2002; Larkin et al., 2013; Savukoski et al., 1998; Schmiedt et al., 2010; Vesa et al., 2002). The function of CLN5 remains to be elucidated, though several in vitro interaction partners have been identified in overexpression systems, including NCL proteins CLN1, CLN2, CLN3, CLN6 and CLN8; the lysosomal sorting receptor sortilin; and small GTPases Rab7 and Rab5 (Basak et al., 2021; Lyly et al., 2009; Mamo et al., 2012; Vesa et al., 2002). 
One study implicated CLN5 in the recruitment of retromer to endosomes via Rab7 and in retrograde trafficking of lysosomal sorting receptors (Mamo et al., 2012). The mechanism by which CLN5 deficiency leads to the disease phenotype is not known.

Thirty-seven pathogenic variants have been reported in all 4 exons and in noncoding regions of CLN5 including missense and nonsense mutations, frameshifts, deletions, insertions and sequence variants ("NCL Mutation and Patient Database," n.d.). The most common variant is the Fin major mutation, pTyr392X, a two base pair deletion predicted to shorten the protein from 407 to 391 amino acids (Savukoski et al., 1998). The Fin major mutation has been found only in Finland where it has a high carrier frequency of 1:24 on the western coastline to 1:100 elsewhere (Åberg et al., 2011). Many other CLN5 variants have been reported in patients from diverse ethnic backgrounds with more variable disease phenotypes (Xin et al., 2010).

The brains of CLN5 patients exhibit progressive atrophy including loss of neurons of the cerebral cortex and thinning of the cortex in the frontal and occipital lobes, extreme cerebellar atrophy with loss of most granule and Purkinje cells, severe degeneration of the thalamus and hippocampus, brainstem atrophy and loss of myelin including thinning of the corpus callosum (Autti et al., 1992; Haltia et al., 2001; Holmberg et al., 2000; Tyynelä et al., 2004, 1997). Storage material accumulates throughout the body; in extraneuronal tissues there is storage but no cell loss. This storage material contains SCMAS as well as sphingolipid activator proteins $A$ and $D$ and the ultrastructure is varied, 
comprising rectilinear and atypical curvilinear profiles as well as fingerprint bodies and granular deposits (Anderson et al., 2013; Tyynelä et al., 1997).

In most cases, the onset of clinical signs occurs in children between 2 and 8 years of age (Holmberg et al., 2000; Simonati et al., 2017; Xin et al., 2010). Cognitive, motor and language function usually decline first followed by seizures and impaired vision progressing to blindness. Cognitive impairment, impaired motor function, visual decline and a decline in language abilities are typical early disease signs. Cognitive decline progresses quickly, and motor dysfunction rapidly progresses from clumsiness to pronounced dysfunction, ataxia, and finally anarthria, difficulties eating and swallowing, loss of independent movement and spastic contractures by 10 to 18 years of age. Seizure onset occurs between 4 and 13 years of age as partial and generalized seizures followed by myoclonus. Other disease signs may include behavior disturbances and sleep alterations (Kirveskari et al., 2001; Simonati et al., 2017). Death typically occurs between 12 and 23 years of age, though some patients survive into their thirties (Simonati et al., 2017).

In most patients, visual failure occurs between 5 and 10 years of age, although a subset of affected individuals with some variants exhibit visual signs that are minimal or begin later in the disease course (Bessa et al., 2006; Ge et al., 2018; Pineda-Trujillo et al., 2005; Santavuori et al., 1982; Simonati et al., 2017; Xin et al., 2010). In two groups of Finnish patients, visual decline first became apparent at $5-8$ years of age, and all children examined were blind by $9-10$ years of age (Santavuori et al., 1991, 1982). In this group of patients, the 
ERG recorded with skin-surface electrodes was extinguished by 8 years of age. These findings indicated that vision loss in this disorder is due at least in part to impaired retinal function. Visual evoked potentials (VEPs) were assessed in affected children to determine whether central nervous system processing of visual signals is altered in this disorder. The first change, pathologically elevated VEPs, occurred early in the preclinical stage of the disease process in some children while in others this was not obvious until $8-9$ years of age. In some of the children the VEP responses then declined, eventually becoming nonrecordable (Santavuori et al., 1991, 1982). Similar results - abolished ERGs at $6-8$ years of age and elevated flash VEPs at $6-10$ years of age - were reported in a separate study which included Dutch and Swedish patients (Holmberg et al., 2000). Children of another cohort of patients from several countries exhibited decreased ERG amplitudes and abnormal VEPs (increased latency and reduced amplitude) (Simonati et al., 2017). Ophthalmoscopic examinations of children with CLN5 disease revealed generalized retinal dystrophy which progressed to optic atrophy at $7-9$ years of age with narrowing of retinal vessels and pigment changes (Santavuori et al., 1991, 1982). Histologically, there is severe degeneration of the neural retina and vision loss in CLN5 disease has been attributed to a progressive decline in retinal function and associated retinal degeneration (Åberg et al., 2011; Tyynelä et al., 1997). However, because CLN5 disease is characterized by widespread brain atrophy, CNS degeneration is also likely to play a significant role in vision loss. 


\subsection{Approaches to Therapy for CLN2 Disease}

The primary strategy for treating monogenetic lysosomal storage disorders like the NCLs is to supply the missing or dysfunctional protein. In the case of a soluble hydrolase like TPP1, extracellular M6P-tagged enzyme can be taken up by cell-surface M6P receptors and delivered to the lysosomes of deficient cells via receptor-mediated endocytosis (Sands and Davidson, 2006). Relatively low levels of enzyme are likely therapeutically relevant; carriers are asymptomatic despite having enzymatic activity reduced from normal, and studies in mice have suggested that less than $10 \%$ of normal levels may be sufficient to confer a therapeutic benefit (Sleat et al., 2008).

The brain and retina are the sites of neuronal loss and are the tissues that require treatment in CLN2 patients. The challenge is in delivering therapeutic protein to these tissues because the enzyme does not cross the blood-brain or blood-retina barriers (Pardridge, 2005). Although these compartments are not accessible from the bloodstream, most of the cells in these tissues are in close proximity to a relevant and accessible extracellular space: the CSF which circulates around and bathes the brain and spinal cord and the vitreous body which comes into contact with the entire surface of the neural retina. Therefore, delivery of a source of TPP1 to the CSF or vitreous can result in wide TPP1 distribution in the CNS and retina respectively (Figure 1-1). In these studies, we tested three methods of delivering TPP1 to the brain and retina via the CSF and vitreous: enzyme replacement therapy, ex vivo cell-mediated gene therapy, and in vivo gene therapy. 


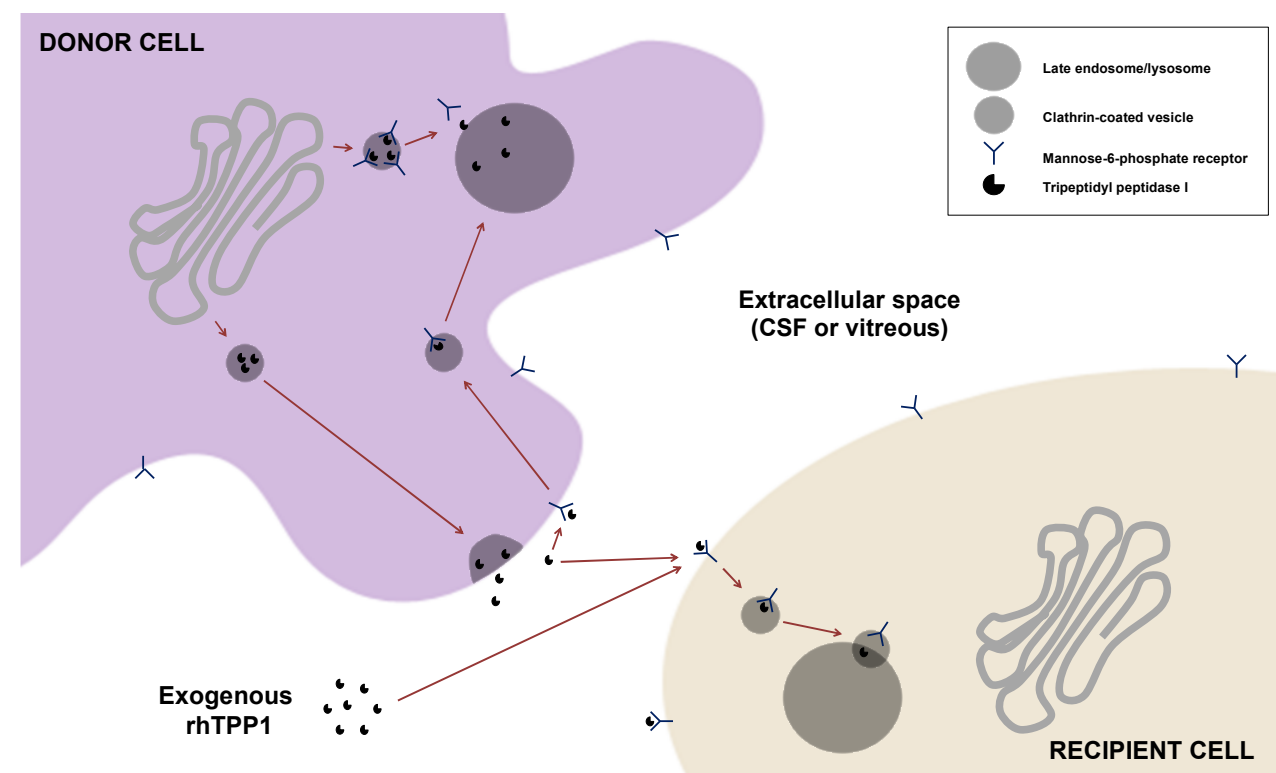

Figure 1-1: TPP1 is trafficked to lysosomes via the M6P pathway; however, some newly synthesized TPP1 protein is instead secreted. Extracellular TPP1 can be taken up after binding to cell-surface M6P receptors and trafficked to the lysosome. Therefore, delivery of M6P-tagged TPP1 can correct the metabolic deficit in TPP1-deficient cells. The source of this extracellular TPP1 could be exogenously delivered recombinant protein or TPP1 secreted by a cell with a functional copy of TPP1.

\subsubsection{Enzyme Replacement Therapy}

Enzyme replacement therapy, the delivery of purified, functional recombinant enzyme to affected tissues, was tested in the Dachshund model of CLN2 disease in collaboration with BioMarin Pharmaceuticals (Katz et al., 2014; Vuillemenot et al., 2011; Whiting et al., 2015). Recombinant human TPP1 protein (rhTPP1) was delivered to the cerebrospinal fluid (CSF) via infusion through catheters into the ventricles and subarachnoid space at L5. Survival was prolonged in treated dogs in a dose-dependent manner and was doubled in a dog treated at the highest dose. Treatment delayed or prevented onset of neurological symptoms and reduced ventricular enlargement (Katz et al., 2014). 
On the basis of the CLN2-affected Dachshund studies, a clinical trial was undertaken in which children with CLN2 disease received infusions of the therapeutic, Cerliponase alfa, via intracerebroventricular (ICV) catheter every two weeks. The treatment successfully halted disease progression, indicated by a reduction in the loss of points on the Hamburg rating scale over the study period, from 2.09 in natural history cases to 0.48 points (Schulz et al., 2018). Cerliponase alfa, or Brineura, was approved by the FDA in 2017 as the first disease-modifying treatment for an NCL and is currently in use worldwide for treating CLN2 disease (Markham, 2017).

However, periodic infusion of rhTPP1 into the CSF of CLN2-affected dogs did not prevent blindness or retinal degeneration. ERG a- and b-waves were abolished and retinal thinning was apparent in both treated and untreated dogs (Whiting et al., 2016). Likewise, retinal function was not preserved in a patient treated with Cerliponase alfa (Rigaudière et al., 2021). Preventing loss of vision in CLN2 disease will likely require delivery of TPP1 to both the brain and retina. Therefore, in collaboration with BioMarin Pharmaceuticals, we tested the ability of intravitreal (IVT) delivery of rhTPP1 to preserve retinal function and structure in the Dachshund model of CLN2 disease (Chapter 2; Whiting et al., 2020a, 2020b).

The major drawback to enzyme replacement therapy in CLN2 disease is the requirement for repeated, lifelong administration of the recombinant protein that is available at a limited number of medical centers and the associated procedure-related risks, time cost and expense. Therefore, alternative strategies 
to provide a long-term source of functional TPP1 to the brain and retina are under investigation. Two potential solutions, described here, are ex vivo cellmediated gene therapy (Chapter 3) and in vivo direct gene therapy (Chapter 4).

\subsubsection{AAV-Mediated Gene Therapy}

\subsubsection{Adeno-Associated Virus}

Several viral vectors have been used for gene transfer, but adenoassociated virus (AAV) has emerged as the most promising candidate for gene therapy. AAV is a single-stranded parvovirus that requires co-infection with a helper virus, such as adenovirus, for expression of its genome. In the absence of a helper virus, AAV cannot replicate. The $\sim 4.7 \mathrm{~kb}$ genome contains two open reading frames, rep and cap, between inverted terminal repeats (ITRs) (Weitzman and Linden, 2011).

For use in gene therapy, the wild-type AAV genome is replaced with an expression cassette consisting of the gene of interest and appropriate regulatory sequences. A limitation of $A A V$ is that the maximum length of this expression cassette is around $5 \mathrm{~kb}$. Only the ITRs from the wild-type AAV genome, which are required in cis, are included in the recombinant AAV ( $\mathrm{rAAV}$ ) sequence (Gray and Zolotukhin, 2011). The other components required for replication and packaging, the proteins produced by the rep and cap genes and a helper virus, are supplied in trans in the packaging system. rAAV is manufactured by transduction of cultured cells with rep/cap, adenovirus and vector construct 
plasmids followed by vector purification (Clément and Grieger, 2016; Naso et al., 2017).

AAV attaches to the surface of the target cell by binding a cell-surface glycan and is thought to then bind a co-receptor which allows entry via endocytosis. Different AAV serotypes are able to enter and transduce different tissues and cell types with varying efficiency due to initial recognition by different cell-surface receptors; this, in turn, is due to differences in the structure of the capsid (Agbandje-McKenna and Kleinschmidt, 2011). Modification of the capsid structure can be engineered to produce variants with desired traits, such as more efficient transduction of certain tissues or immune evasion (Kotterman and Schaffer, 2014). After entry into the cell, the viral particle is trafficked to the nucleus, uncoated, and the genome is released from the capsid. While wild-type $A A V$ integrates into the genome in a site-specific manner on chromosome 19, DNA delivered by rAAV used for gene transfer applications persists as circular extrachromosomal episomes. Non-targeted integration has been detected but is reliant on rep proteins not present in the rAAV genome and occurs at a low frequency (Agbandje-McKenna and Kleinschmidt, 2011; Smith, 2008).

Several features make $A A V$ attractive for clinical gene transfer: $A A V$ is replication-deficient and is not associated with human disease; rAAV is largely non-integrating and not associated with insertional mutagenesis; AAV is capable of infecting both dividing and non-dividing cells; and AAV serotypes show different tissue tropisms, allowing some degree of targeting, and capsid engineering can improve desired properties. In post-mitotic or slowly dividing 
cells, viral episomes mediate long-term gene expression (Berns and Muzyczka, 2017).

The target tissue, route of delivery, dose and expression cassette design, including the choice of promoter sequence, must be taken into account when designing a gene therapy experiment. Because TPP1 is ubiquitously expressed and we wanted to target all cell types in the target tissues, we chose to use the CAG promoter, a combination of the cytomegalovirus early enhancer element and the chicken beta-actin promoter that results in ubiquitous overexpression of the transgene. A strong promoter may also reduce the dose needed to achieve a therapeutic effect and thereby reduce the risk of inflammation which is related to dose. Target tissue and route of delivery are discussed in detail below and dosing is discussed in the results.

\subsubsection{Vector Delivery: In vivo versus ex vivo Gene Therapy}

Both in vivo and ex vivo gene therapy is feasible for CLN2 disease. Direct, or in vivo, gene therapy involves administration of rAAV particles and in vivo transduction of the patient's cells. The choice of rAAV serotype and method of injection are critical for direct gene therapy applications as they dictate vector distribution and which tissues and cell types will be transduced. Advantages of direct gene therapy are the ability to transduce large numbers of cells with a single injection and the potential for long-term transgene expression. Episomal transgene DNA may be diluted and lost in highly proliferative tissues, but expression in adult post-mitotic cells and tissues appears to persist for at least several years (Berns and Muzyczka, 2017). However, exposure to wild-type AAV 
and seropositivity for anti-AAV antibodies is common and it is a concern that preexisting neutralizing antibodies (NAbs) could limit transduction (Calcedo et al., 2009; Erles et al., 1999). In addition, the development of anti-AAV antibodies after treatment precludes the possibility of repeated administration of the same serotype should transgene expression decline over time. Another concern is that use of cells' translational machinery to overexpress the transgene could potentially impair the normal functions of transduced cells.

Ex vivo transduction and implantation of transduced cells is an alternative to direct injection of rAAV particles (Gowing et al., 2017; Gregory-Evans et al., 2012; Tracy et al., 2016). Some TPP1 protein is naturally secreted by the cells (Figure 1-1). Implanted TPP1-overexpressing cells are able to secrete functional TPP1 into the extracellular space and provide TPP1 to resident cells. Because rAAV particles are applied prior to implantation and are not seen by the immune system, pre-existing immunity is not a barrier to successful treatment and repeat administration is possible. An additional benefit to ex vivo gene therapy with mesenchymal stem cells is the immunomodulatory properties of these cells, discussed in 1.2.2.4. However, the distribution of implanted cells is difficult to track and the long-term survivability of implanted cells is unknown. An additional technical limitation to this technique is the need to obtain a sufficient number of cells for injection.

\subsubsection{In vivo Gene Transfer to the Retina}

rAAV2 has been the vector of choice for retinal gene therapy and is the serotype used in the only FDA-approved retinal gene therapy, voretigene 
neparvovec (Bainbridge et al., 2015; Cukras et al., 2018; Dimopoulos et al., 2018; Heier et al., 2017; Russell et al., 2017). The two primary methods for administration of drugs to the eye intended to reach the retina are subretinal and intravitreal injection (Padhy et al., 2020). Subretinal injection is a technically challenging procedure that produces a temporary detachment of the retina into which the drug is injected, and the surgery alone can cause complications including retinal detachments and retinal tears (Gregori and Davis, 2020). Injection into the vitreous, a gelatinous, avascular and largely acellular material that makes up the center of the eye, is a relatively straightforward procedure that can be performed by most ophthalmologists with minimal specialized training and is routinely used on a monthly basis in an outpatient setting to deliver treatment for age-related macular degeneration in human patients (Maguire et al., 2016). Subretinal and intravitreal injections of rAAV result in different patterns of transgene expression. Subretinal rAAV2 requires only a very low dose of vector and primarily transduces photoreceptors and cells of the RPE limited to the area of the subretinal bleb formed during injection (Narfström et al., 2003;

Vandenberghe et al., 2011). Intravitreal administration requires a higher dose and results in less efficient but more widespread transduction of inner retinal cells, especially ganglion cells (Yin et al., 2011). There is a concern that vector injected intravitreally may not penetrate retinal layers as effectively due to the barrier of the inner limiting membrane. In addition, photoreceptors and the RPE are not targeted by this delivery method except perhaps at very highest doses (Dalkara et al., 2009; Mowat et al., 2014). However, as secreted TPP1 is 
therapeutic, it is probably not necessary to transduce every cell in the retina. Because the inner retina is affected early and most severely in both human and canine CLN2 disease, we chose to test the intravitreal route of administration. Additionally, because the degenerating retina may be fragile, especially in CLN2affected dogs which develop retinal detachment lesions as part of the disease process, we thought it was important to avoid interrupting the attachment of the retina to the underlying epithelium.

\subsubsection{Ex vivo Gene Therapy: Genetically Modified Mesenchymal Stem Cells as a Drug Delivery System}

Mesenchymal stem cells (MSCs, also called multipotent mesenchymal stromal cells) are a multipotent, self-renewing population of adult stem cells capable of differentiating into and regenerating mesodermal tissues such as bone, fat and cartilage. MSCs have been isolated from multiple tissues, primarily bone marrow, adipose tissue and umbilical cord. MSCs are easily and rapidly isolated from bone marrow (BM-MSCs) and expanded and maintained in culture, and large-scale production of clinical-grade BM-MSCs is already underway, making them ideal candidates for therapeutic use (Lechanteur et al., 2016).

The Mesenchymal and Tissue Stem Cell Committee of the International Society for Cellular Therapy proposed three defining features of MSC: (1) plastic adherence under standard culture conditions; (2) by flow cytometry, greater than 95\% of the population is positive for expression of CD105, CD73 and CD90 and less than $2 \%$ of the population is negative for CD45, CD34, CD14, CD11b, CD79 $\alpha$, CD9 and HLA-II; and (3) differentiation in vitro into chondroblasts, 
adipocytes and osteoblasts (Dominici et al., 2006). In culture, they have a fibroblast-like morphology. Plastic adherence is commonly used as a method of isolating MSCs from a mixed population of cells; over time in culture, nonadherent cell types are washed away, leaving predominately MSCs. The ISCTdefined positive surface markers are not unique to MSCs so it must also be demonstrated that MSCs do not express characteristic surface markers of leukocytes, antigen presenting cells, hematopoietic progenitors, endothelial cells, monocytes/macrophages and B cells. This panel is not unique to MSCs; therefore, demonstration of trilineage potential in vitro is the final defining feature of cultured MSCs.

Clinical interest lies in the MSCs' ability to migrate to sites of injury or inflammation and exert a number of regenerative mechanisms, including immunomodulation and neuroprotection (Lam et al., 2013; Squillaro et al., 2016). MSCs interact with and modulate cells of both the innate and adaptive immune systems (Gao et al., 2016; Uccelli et al., 2008). The mechanisms of MSC immunomodulation are not fully understood but are carried out by both soluble and contact-dependent factors. The secretome of MSCs includes neurotrophic factors, cytokines, chemokines, proteins and extracellular vesicles, which may themselves contain chemokines, cytokines, growth factors, anti-inflammatory factors and RNAs. The in vivo microenvironment experienced by implanted MSCs is thought to modulate the secretome, so their therapeutic immunomodulatory effects may vary dependent upon disease state (Eleuteri and Fierabracci, 2019). Neuroprotective effects and promotion of cell survival are 
likely mediated by the secretion of neurotrophic factors and anti-oxidant molecules (Crigler et al., 2006; Kemp et al., 2010; Stemberger et al., 2011; Uccelli et al., 2011; Wilkins et al., 2009). Neuroprotective, anti-inflammatory and anti-apoptotic effects may also be due in part to regulation of microglia through cytokine release and immune modulation (Giunti et al., 2012; Hsuan et al., 2016; Uccelli et al., 2011).

Transplantation of MSCs has shown promise in animal models of neurological and retinal disorders and is under investigation in numerous clinical trials, though most trials are ongoing and few results are available (Squillaro et al., 2016). In the eye, intravitreal, subretinal, sub-Tenon and intravenous administration of MSCs have been used in clinical trials of retinitis pigmentosa and diabetic retinopathy (Gu et al., 2018; Labrador-Velandia et al., 2016; Oner et al., 2016; Özmert and Arslan, 2020; Satarian et al., 2017). In the CNS, MSCs have been used intravenously, intraparanchymally and intrathecally in patients with stroke, multiple sclerosis and amyotrophic lateral sclerosis (Connick et al., 2011; Díez-Tejedor et al., 2014; Karussis et al., 2010; Shichinohe et al., 2017; Syková et al., 2017).

MSCs also have a potential use as a delivery system for therapeutic molecules. The innate immunomodulatory properties of MSCs make them an ideal delivery system for therapeutic protein to the retina because intraocular inflammation is a concern for both enzyme replacement and in vivo gene therapies. For lysosomal storage diseases caused by lack of a specific soluble enzyme, implantation of allogenic MSCs lacking a disease-causing mutation or 
autologous MSCs genetically engineered to express a functional copy of the gene can supply a long-term source of functional protein to target tissues. Studies conducted in our laboratory in the canine model of CLN2 disease demonstrated that intravitreal implantation of genetically modified autologous BM-MSCs overexpressing human TPP1 (hTPP1) is an effective method of delivering therapeutic protein to the retina and inhibiting retinal degeneration (Tracy et al., 2016). BM-MSCs were transduced with AAV2.CAG.hTPP1 (TPP1MSCs) or AAV2.CAG.GFP (GFP-MSCs) vector prior to implantation in the vitreous of CLN2-affected dachshunds. Clusters of GFP-MSCs could be identified in enucleated eyes up to 9 weeks following implantation. Histological examination indicated that the implanted cells retained a fibroblast-like morphology and embedded in an extracellular matrix. GFP-MSCs were not observed to adhere to the posterior lens capsule or the vitreal surface of the retina or to integrate into the retina. While control eyes treated with GFP-MSCs demonstrated typical disease progression, CMR lesions were reduced or prevented, ERG amplitudes preserved, and normal retinal morphology maintained through 10 months of age in eyes treated TPP1-MSCs. In the studies described here, we worked to further optimize this treatment and to use TPP1MSCs administered to the CSF to treat neurological disease signs.

\subsection{Study Objectives}

The most promising mode of treatment for CLN2 disease is delivery of functional TPP1 protein to the brain and retina to correct the metabolic deficit. These tissues are not accessible to TPP1 in the bloodstream due to the blood- 
brain and blood-retinal barriers, so targeted methods of delivery are required. Because of the quick turnover of TPP1, either repeated dosing or a long-term source of enzyme is needed. There are several possible methods to achieve this goal. Herein we tested three methods: enzyme replacement therapy, ex vivo gene therapy and in vivo gene therapy. Any of these methods could be optimized for use in children with CLN2 disease. Moreover, these approaches could be applied to other forms of NCL or indeed any lysosomal storage disorder that is due to a deficit of a soluble lysosomal protein, including the CLN1, CLN5, CLN10 and CLN13 forms of NCL. We therefore also characterized visual system pathology in a canine disease model and collected baseline data which could serve as a foundation for conducting therapeutic intervention studies for CLN5 disease similar to those done in the CLN2 disease model.

The specific aims for this study are as follows:

1. Evaluate the safety and efficacy of repeated intravitreal dosing of rhTPP1 to preserve retinal structure and function and delay ophthalmic disease progression in the canine model of CLN2 disease.

2. Evaluate the safety and efficacy of intravitreal and CSF administration of TPP1-MSCs to provide a long-term source of TPP1 and prevent ophthalmic and neurological disease progression in the canine model of CLN2 disease.

3. Evaluate the safety and efficacy of in vivo retinal gene therapy to provide a long-term source of TPP1 and prevent ophthalmic disease progression in the canine model of CLN2 disease. 
4. Characterize ocular disease progression in Golden Retrievers with CLN5 disease to develop a model that can be used in preclinical studies of therapies targeting the retina. 


\section{Intravitreal Enzyme Replacement Therapy Preserves Retinal Structure and Function in the Canine Model of CLN2 Disease}

\subsection{Introduction}

Delivery of rhTPP1 to the CSF is an effective method for delaying or preventing the onset of neurologic symptoms of CLN2 disease and is already an approved therapy for CLN2 disease (Katz et al., 2014; Markham, 2017; Schulz et al., 2018). However, vision loss in human and canine CLN2 disease is due to degeneration of both visual pathways in the brain and the retina, and rhTPP1 administered to the CSF does not reach the retina in sufficient amounts to prevent disease-related retinal degeneration. Dogs receiving bi-weekly CSF infusions of rhTPP1 exhibited decline in ERG amplitudes and retinal degeneration similar to that seen in untreated dogs (Whiting et al., 2016). Thus, additional therapy targeting the retina is required to preserve vision in patients treated with enzyme replacement therapy via CSF delivery.

Intravitreal (IVT) injection of therapeutic proteins to target the retina is an established treatment for posterior segment diseases of the eye (Ba et al., 2015; Park et al., 2016; Radhakrishnan et al., 2017). Injected proteins have a half-life of approximately one week in the vitreous, depending on the molecular weight of the protein and the size of the eye, and a significant proportion of the injected protein is transported from the vitreous to the retina (del Amo et al., 2017; Park et 
al., 2016; Radhakrishnan et al., 2017). We hypothesized that intravitreal injection of rhTPP1 could supply functional TPP1 to the retina and prevent or delay retinal degeneration and loss of function. The goal of this study was therefore to evaluate whether periodic intravitreal administration of rhTPP1 initiated prior to or after the onset of detectable declines in retinal function is effective at preventing or slowing the progression of disease-related retinal degeneration and loss of function in the canine model of CLN2 disease.

\subsection{Methods}

\subsubsection{Animals}

The dogs used for this study were TPP1/- purpose-bred Dachshunds $(n=8)$ housed in a research colony at the University of Missouri - Columbia. The research colony was established in 2005 from the breeding of a pair of miniature longhaired Dachshunds heterozygous for a single nucleotide deletion in exon 4 of TPP1. Affected dogs were generated by breeding $T P P 1^{+/-}$females to $T P P 1^{+/-}$ or $\mathrm{TPP}^{-/-}$males with no common ancestor for at least the two preceding generations. Unrelated carrier males from outside the colony were periodically bred to colony $T P P 1^{+-}$or $T P P 1^{-/}$dogs to minimize inbreeding. Dogs were implanted with microchips for identification at approximately one week of age. Prior to enrollment in the study, dogs were genotyped at the TPP1 locus using an allelic discrimination assay. Buccal cells were collected on FTA Elute cards (GE Whatman, Pittsburg, PA) via a cheek swab. Samples were allowed to air dry completely and stored at room temperature prior to use. DNA was eluted from 
the cards by removing three 3-mm punches from the card, washing with water, adding $40 \mu \mathrm{L}$ of $18.2 \mathrm{M} \Omega . \mathrm{cm}$ ultrapure water and incubating at $95^{\circ} \mathrm{C}$ for 30 minutes on a thermal block. $10 \mu \mathrm{L}$ of eluted buccal cell DNA was added in duplicate to tubes containing $10.9 \mu \mathrm{L}$ ultrapure water, $12.5 \mu \mathrm{L}$ TaqMan Genotyping Master Mix (Applied Biosystems, Foster City, CA) and 0.625 $\mu \mathrm{L} 40 \mathrm{X}$ custom CLN2 TaqMan SNP Genotyping Assay mix (Assay ID AH1RT9C; Applied Biosystems, Foster City, CA). The assay mix contains fluorescein dye-labeled primer (forward: ACCTTCCGCACAGTCCAAAA; reverse:

AGCCAGCAAGTCAGAAAGTCTT) and probe (c.325del:

AGTTCCGGGCTCCAGC; wild type: CAGTTCCGGCTCCAGC) sets that recognize the wild type and c.325deIC TPP1 alleles. $1 \mu \mathrm{L}$ of DNA extracted from blood of known TPP1+/+, TPP1/- and TPP1+/- animals was added to positive control wells and $10 \mu \mathrm{L}$ ultrapure water was added to negative control wells. PCR amplification and endpoint detection were performed on an Applied Biosystems StepOne Real-Time PCR system. Dogs were maintained on a 12:12 daily light cycle, received routine husbandry and veterinary care and were socialized daily. The study was performed in compliance with the ARVO Statement for the Use of Animals in Ophthalmic and Vision Research and was approved by the University of Missouri Animal Care and Use Committee.

\subsubsection{Ophthalmic Examinations}

At approximately 10-12 weeks of age, prior to enrollment in the study,

dogs received a complete eye exam and dogs with any ophthalmic abnormalities that could compromise vision were excluded from the study. Ophthalmic exams 
included assessment of visually mediated behavior, menace response and pupillary light reflex (PLR). Pupils were then dilated with $1 \%$ tropicamide ophthalmic solution (Alcon, Fort Worth, TX) and slit lamp biomicroscopy (SL14; Kowa Co. Ltd., Tokyo, Japan) and indirect ophthalmoscopy were performed. Indirect ophthalmoscopy was done with an indirect wireless headset $(12,500$; Welch Allyn Inc., Skaneateles Falls, NY) and a 30 diopter clear handheld lens (Volk Optical Inc., Mentor, OH). In addition to the pre-enrollment examination, ophthalmic examinations were performed one day and one week after each treatment, and then monthly and as needed to assess complications.

\subsubsection{Treatments}

All animals enrolled in the study received recombinant human TPP1 (rhTPP1; BioMarin Pharmaceutical, Novato, CA) by injection into the vitreous of the left eye (OS) and an equivalent volume of vehicle by injection into the vitreous of the right eye (OD). rhTPP1 was produced as described previously (Lin and Lobel, 2001). The vehicle for Dogs E1, E2 and E3 was an intravitreal formulation (10 mM sodium phosphate, 10\% Trehalose, $\mathrm{pH}$ 6.5; BioMarin Pharmaceuticals), while the vehicle for Dogs E4, L1, L2, L3 and L4 was the artificial CSF formulation (1 $\mathrm{mM}$ sodium phosphate, $3 \mathrm{mM} \mathrm{KCl}, 1.4 \mathrm{mM} \mathrm{MgCl}_{2}$, $0.8 \mathrm{mM} \mathrm{CaCl}_{2}$, and $148 \mathrm{mM} \mathrm{NaCl}, \mathrm{pH}$ 6.5; BioMarin Pharmaceuticals) previously used in the central nervous system (CNS) enzyme replacement studies and approved for use via ICV administration in children with CLN2 disease.

Prior to each injection, dogs were sedated with dexmedetomidine up to 20 $\mu \mathrm{g} / \mathrm{kg}$ and $0.015 \mathrm{mg} / \mathrm{kg}$ buprenorphine. One drop each of $0.3 \%$ ofloxacin and 
$0.5 \%$ proparacaine hydrochloride was applied to each eye and the ocular surface was cleaned with 1:50 dilute povidone-iodine. For all injections, the dorsolateral bulbar conjunctiva was grasped with forceps and the globe was rotated ventromedially. Intravitreal injection was performed with the attached 0.5 inch, 28 or 29 gauge needle of a $0.5 \mathrm{~mL}$ insulin syringe inserted $5-7 \mathrm{~mm}$ posterior to the limbus directed posterior to the lens. One drop of ofloxacin was applied to each eye following the injection. Intraocular pressure (IOP) measurements were performed prior to and following injection.

Initiation of treatments at both pre- and post-symptomatic ages was tested since treatment can slow or arrest but likely not reverse disease progression. Currently, diagnosis and initiation of treatment shortly after birth is not feasible except in the case of affected siblings, so it was important to test both early and late intervention. Pre-symptomatic early start treatment for dogs E1, E2, E3 and E4 began at approximately 12 weeks ( 3 months) of age, prior to the onset of any detectable deficits in retinal function. Post-symptomatic late start treatment of dogs L1, L2, L3 and L4 began at 22-25 weeks (approximately 5.5 months) of age, just after CLN2-affected dogs begin to exhibit detectable declines in retinal function. The dosing regimen for each dog was individualized as adjustments were made on the basis of preliminary results from previously treated dogs, complications from intraocular inflammation and ongoing ERG results.

We initially planned to treat early start dogs E1-E4 with biweekly intravitreal injections of rhTPP1 or vehicle with a starting dose of $0.1 \mathrm{mg}$ rhTPP1 at 12 weeks of age followed by a dose escalation. However, the treated eye of 
the first dog (Dog E1) exhibited severe ocular inflammation after escalation from 0.1 to $0.3 \mathrm{mg}$, so no further dose escalations were performed and, with the exception of this $0.3 \mathrm{mg}$ dose, all doses for all dogs were $0.1 \mathrm{mg}$. The timing of dosing was adjusted for each dog in an ongoing manner to mitigate adverse effects and to determine the optimal frequency of dosing. Individual dosing schedules for each dog are shown in Table 2-1.

\begin{tabular}{|c|c|c|c|c|c|c|c|c|c|c|c|}
\hline \multicolumn{12}{|c|}{ Pre-Symptomatic ERT Dosing Schedule } \\
\hline \multicolumn{3}{|c|}{ Dog E1 } & \multicolumn{3}{|c|}{ Dog E2 } & \multicolumn{3}{|c|}{ Dog E3 } & \multicolumn{3}{|c|}{ Dog E4 } \\
\hline $\begin{array}{c}\text { Age } \\
\text { (months) }\end{array}$ & $\begin{array}{c}\text { Week of } \\
\text { treatment }\end{array}$ & $\begin{array}{l}\text { Dose } \\
\text { (mg) }\end{array}$ & $\begin{array}{c}\text { Age } \\
\text { (months) }\end{array}$ & $\begin{array}{c}\text { Week of } \\
\text { treatment }\end{array}$ & $\begin{array}{l}\text { Dose } \\
\text { (mg) }\end{array}$ & $\begin{array}{c}\text { Age } \\
\text { (months) }\end{array}$ & $\begin{array}{c}\text { Week of } \\
\text { treatment }\end{array}$ & $\begin{array}{l}\text { Dose } \\
\text { (mg) }\end{array}$ & $\begin{array}{c}\text { Age } \\
\text { (months) }\end{array}$ & $\begin{array}{c}\text { Week of } \\
\text { treatment }\end{array}$ & $\begin{array}{l}\text { Dose } \\
(\mathrm{mg})\end{array}$ \\
\hline 3 & 1 & 0.1 & 2.5 & 1 & 0.1 & 2.8 & 1 & 0.1 & 3.1 & 1 & 0.1 \\
\hline 3.4 & 3 & 0.1 & 3 & 3 & 0.1 & 3.2 & 3 & 0.1 & 4.1 & 5 & 0.1 \\
\hline 3.9 & 5 & 0.3 & 3.4 & 5 & 0.1 & 3.7 & 5 & 0.1 & 5.5 & 11 & 0.1 \\
\hline 5.2 & 11 & 0.1 & 3.9 & 7 & 0.1 & 4.1 & 7 & 0.1 & 6.8 & 17 & 0.1 \\
\hline \multicolumn{3}{|c|}{ Treatment discontinued due } & 4.3 & 9 & 0.1 & 4.6 & 9 & 0.1 & 8.3 & 23 & 0.1 \\
\hline \multicolumn{3}{|c|}{ to intraocular inflammation. } & 4.9 & 11 & 0.1 & 5.2 & 12 & 0.1 & \multirow{2}{*}{\multicolumn{3}{|c|}{ Dosing Frequency }} \\
\hline & & & 5.5 & 14 & 0.1 & 6 & 15 & 0.1 & & & \\
\hline & & & 6.2 & 17 & 0.1 & 6.6 & 18 & 0.1 & \multicolumn{3}{|c|}{ Every 2 weeks } \\
\hline & & & 6.9 & 20 & 0.1 & 8 & 24 & 0.1 & \multicolumn{3}{|c|}{ Every 3 weeks } \\
\hline & & & 7.5 & 23 & 0.1 & & & & \multicolumn{3}{|c|}{ Every 4 weeks } \\
\hline & & & 8.2 & 26 & 0.1 & & & & \multicolumn{3}{|c|}{ Every 6 weeks } \\
\hline
\end{tabular}

Table 2-1: Pre-symptomatic enzyme replacement therapy dosing schedule. Grey coloring indicates length of interval between doses.

Late start dogs L1-L4 received an initial dose of $0.5 \mathrm{mg}$ at $23-25$ weeks of age. A second dose of $0.25 \mathrm{mg}$ was administered to Dogs L1 and L4 when monthly ERG recordings determined that b-wave amplitudes had declined by an amount greater than $20 \%$ of baseline b-wave amplitudes. Dog L3 did not receive a second dose at this point due to the severity of neurologic disease signs. Dog L2 was given a second injection 8 weeks after the first even though there was no 
decline in ERG amplitudes at that point, in order to test the feasibility of redosing with larger amounts than previously tested at this point. Individual dosing schedules for each dog are shown in Table 2-2.

\begin{tabular}{|c|c|c|c|c|c|c|c|c|c|c|c|}
\hline \multicolumn{12}{|c|}{ Post-Symptomatic ERT Dosing Schedule } \\
\hline \multicolumn{3}{|c|}{ Dog L1 } & \multicolumn{3}{|c|}{ Dog L2 } & \multicolumn{3}{|c|}{ Dog L3 } & \multicolumn{3}{|c|}{ Dog L4 } \\
\hline $\begin{array}{c}\text { Age } \\
\text { (months) }\end{array}$ & $\begin{array}{c}\text { Week of } \\
\text { treatment }\end{array}$ & $\begin{array}{l}\text { Dose } \\
\text { (mg) }\end{array}$ & $\begin{array}{c}\text { Age } \\
\text { (months) }\end{array}$ & $\begin{array}{c}\text { Week of } \\
\text { treatment }\end{array}$ & $\begin{array}{l}\text { Dose } \\
(\mathrm{mg})\end{array}$ & $\begin{array}{c}\text { Age } \\
\text { (months) }\end{array}$ & $\begin{array}{c}\text { Week of } \\
\text { treatment }\end{array}$ & $\begin{array}{l}\text { Dose } \\
\text { (mg) }\end{array}$ & $\begin{array}{c}\text { Age } \\
\text { (months) }\end{array}$ & $\begin{array}{c}\text { Week of } \\
\text { treatment }\end{array}$ & $\begin{array}{l}\text { Dose } \\
(\mathrm{mg})\end{array}$ \\
\hline 5.3 & 1 & 0.5 & 5.8 & 1 & 0.5 & 5.6 & 1 & 0.5 & 5.3 & 1 & 0.5 \\
\hline 9 & 17 & 0.25 & 7.6 & 9 & 0.25 & & & & 8.1 & 13 & 0.25 \\
\hline
\end{tabular}

Table 2-2: Post-symptomatic enzyme replacement therapy dosing schedule

\subsubsection{Immunosuppression and Inflammation Control}

All dogs received oral immunosuppressants for the duration of the study. Early start dogs E1, E2 and E3 began twice-daily administration of $25 \mathrm{mg}$ cyclosporine (25 mg capsules USP modified, TEVA Pharmaceuticals, North Wales, PA) one week prior to the initial injection. The dose was adjusted to 35 mg (35 mg compounded cyclosporine capsules, Wedgewood Pharmacy, Swedesboro, NJ) twice a day after the dogs reached a body weight of $4 \mathrm{~kg} .2 .5$ $\mathrm{mg} / \mathrm{kg}$ leflunomide was administered once daily starting 21 (dog E1), 19 (dog E2) and 17 (dog E3) weeks after the first injection. Daily dosing was maintained for 2 months then reduced to every other day. Dog E4 began cyclosporine and leflunomide dosing 3 weeks prior to the first injection. Late start dogs L1-L4 began $35 \mathrm{mg}$ twice-daily cyclosporine and $10-12 \mathrm{mg}$ once-daily leflunomide at approximately 4 months of age, prior to the first injection. All oral immunosuppressants were maintained throughout the study period. 
Topical corticosteroid (0.05\% difluprednate, Durezol, Alcon; or $1 \%$ prednisolone acetate) and non-steroidal anti-inflammatory ( $0.1 \%$ nepafenac, Nevanac, Alcon; or $0.03 \%$ flurbiprofen sodium) drops were administered to each eye beginning 24 hours before each injection. Durezol was administered four times daily and Nevenac was administered three times daily for at least three days after each injection. Additional topical medications (Durezol, Nevenac, prednisolone, flurbiprofen or carprofen) were administered as needed for ocular inflammation and were discontinued when inflammation resolved.

Early start dogs received a sub-Tenon's injection of $2 \mathrm{mg}$ of triamcinolone acetonide (Kenalog-10, Bristol-Myers Squibb, Princeton, NJ) in both eyes immediately following every other injection of rhTPP1. Late start dogs received sub-Tenon's $2 \mathrm{mg}$ triamcinolone injections immediately following each rhTPP1 injection.

\subsubsection{Electroretinography}

Initial baseline bilateral ERG recordings were performed at approximately 3 months of age prior to the first injection of rhTPP1 and monthly thereafter. ERGs were always recorded a minimum of one week after an injection. Additional baseline measurements were performed one week prior to the first rhTPP1 injection in late start dogs.

All ERG procedures were performed in a room with a double door and all light-emitting sources that could not be eliminated covered with red plastic, and all preparation was done with the room lights on. Pupils were dilated with one 
drop of $1 \%$ tropicamide approximately 30 minutes before sedation and were checked to verify adequate and symmetrical dilation. The dog was then sedated with up to $40 \mu \mathrm{g} / \mathrm{kg}$ intramuscular (IM) dexmedetomidine and the intravenous (IV) catheter was placed. Once sedation set in, up to $5 \mathrm{mg} / \mathrm{kg}$ IM ketamine was given to all dogs up to 7 months of age; at 8 months of age and older, ketamine was discontinued to reduce seizure risk. $0.5-2 \mathrm{mg} / \mathrm{kg}$ IV propofol was given to latestage dogs to mitigate disease-related myoclonic jerks. The dog was placed in sternal recumbency on a heating pad and the head was supported by a deflatable cushion so that the nose was positioned slightly downward. One drop of proparacaine was administered to both eyes $(\mathrm{OU})$ as a topical anesthetic. Subdermal ground and reference needle electrodes were then placed. The ground electrode was inserted under the skin over the rostral aspect of the occipital protuberance. Bilateral reference electrodes were inserted halfway between the lateral canthus of the eye and the base of the ear. Conjunctival stay sutures were then placed at the top of the eye near the limbus. A lid speculum was inserted in each eye, with the sutures passing through the top arm. Hemostats were used to clamp the taped end of the sutures and hold the eye in position such that the pupils were centered in the palpebral fissure. A drop of methylcellulose was placed in the cup of ERG-Jet gold mylar film contact lens electrodes (OcuScience, Henderson, NV) and the lenses were placed on the cornea, ensuring good contact. Recordings were performed with a bilateral fullfield flash ERG unit (HMsERG model 1000, RetVet Corp., Columbia, MO). The mini-Ganzfeld domes were positioned about an inch from the dog's eyes, as 
close as the muzzle allowed, ensuring that the domes were centered on the pupils. To check electrode set-up, a $3 \mathrm{~cd} / \mathrm{m}^{2}$ test flash was administered with the room lights still on. The heating pad was disconnected before beginning the ERG protocol. All lights were then turned off at the same time that the ERG protocol was begun. The only light sources allowed in the room during ERG recording were red flashlights and the screen of the ERG equipment. The 'Dog Diagnostic Protocol' recommended by the European College of Veterinary Medicine was used (Ekesten et al., 2013). A dark-adapted ERG to elicit scotopic low-intensity rod responses was performed first. A dark-adaptation period of 4 minutes was initiated as the room lights were turned off and was followed by ten $10.2 \mathrm{log}$ photons $/ \mathrm{cm}^{2} / \mathrm{s}\left(10 \mathrm{mcd} / \mathrm{m}^{2}\right)$ flashes. This was repeated 5 times, for a total darkadaptation period of 20 minutes. At the end of the dark adaptation period, scotopic mixed rod and cone responses were elicited with four 12.65 photons $/ \mathrm{cm}^{2} / \mathrm{s}\left(3 \mathrm{~cd} / \mathrm{m}^{2}\right)$ flashes and, 30 seconds later, four $13.2 \mathrm{log}$ photons $/ \mathrm{cm}^{2} / \mathrm{s}\left(10 \mathrm{~cd} / \mathrm{m}^{2}\right)$ flashes. Dome lights were then turned on (13.65 log photons $/ \mathrm{cm}^{2} / \mathrm{s}, 30 \mathrm{~cd} / \mathrm{m}^{2}$ ) and the dog was light adapted for 10 minutes. Immediately after the light adaptation period, photopic cone responses were elicited with $12.65 \mathrm{log}$ photons $/ \mathrm{cm}^{2} / \mathrm{s}\left(3 \mathrm{~cd} / \mathrm{m}^{2}\right)$ flashes and a $3 \mathrm{~cd} / \mathrm{m}^{2}, 30 \mathrm{~Hz}$ flicker.

ERG recordings were analyzed with ERGView software (OcuScience, Henderson, NV). The average response of each set of flashes was used for analysis. The b-wave amplitude and implicit time were measured for all responses and a-wave amplitude and implicit time were measured for scotopic 
mixed rod and cone and photopic cone responses. Program-assigned a- and bwave amplitude measurements were reviewed and adjusted manually to ensure that the a-wave amplitude was measured from baseline to the trough of the awave and the b-wave amplitude from the a-wave trough to the peak of the bwave. Implicit times were measured as time-to-trough/time-to-peak. The a- and b-wave amplitude measurements and implicit times were exported as a text file and imported into Microsoft Excel for analysis. All traces were exported as .xy files and imported into Excel for visualization. At each time point, b-wave amplitudes were compared between the rhTPP1-treated eye and the vehicle control eye. Historical data from normal $(n=7)$ and affected $(n=6)$ untreated dogs from the same research colony were used for comparison.

\subsubsection{Retinal Imaging}

Beginning at approximately 3 months of age, in vivo scanning laser ophthalmoscopy (SLO) and optical coherence tomography of the retina (OCT) were performed monthly with a Heidelberg Spectralis HRA/OCT (Heidelberg, Germany) with a wide-angle $55^{\circ}$ lens. Typically, SLO/OCT imaging was performed immediately following ERG recording. Eyes were dilated with 1\% tropicamide and the dog sedated with IM dexmedetomidine if this was not already done for the ERG. The dog was anesthetized with IV propofol, intubated with a cuffed endotracheal tube and restraint was maintained with isoflurane in oxygen during imaging. Wide-angle SLO images of the fundus were obtained and used to quantify CMR lesions. Serial OCT scans of the superior and inferior retina as well as the optic nerve head were acquired. These OCT images could 
be used to confirm the presence of CMR lesions seen on fundic images and to visualize inflammation.

\subsubsection{Euthanasia and Necropsy}

Dogs were euthanized when they reached end-stage neurological disease between 9 and 10.5 months (40-46 weeks) of age. End-stage disease was defined by the progression of neurological signs to the point that the dogs were no longer able to eat without assistance and suffered from such severe ataxia that they were in danger of injuring themselves from falling or bumping into obstacles. Final ERG and retinal imaging procedures were performed as described in 2.2.5 and 2.2.6 and the dogs were then euthanized while still sedated. Euthanasia was performed via intravenous administration of $1 \mathrm{~mL}$ per 5 pounds of IV pentobarbital sodium. Death was confirmed by cardiac auscultation.

Both eyes with a portion of the optic nerve attached were immediately enucleated. The eyes from early start dogs were immediately flushed with $2.5 \%$ glutaraldehyde fixative (2.5\% glutaraldehyde/0.1 M sodium cacodylate $/ 1 \mathrm{mM}$ $\mathrm{CaCl}_{2}, \mathrm{pH}$ 7.4), the cornea, iris and lens were removed and the eyecups were placed in vials of $2.5 \%$ glutaraldehyde fixative. Aqueous and vitreous samples were collected from late start dogs. Approximately $0.5 \mathrm{~mL}$ of aqueous humor was withdrawn from the aqueous chamber with a needle and syringe inserted through the limbal cornea. The cornea, iris and lens were removed, and vitreous humor was collected with a plastic transfer pipette and cut away from the eyecup with surgical scissors. The eyecups were then flushed with $2.5 \%$ glutaraldehyde fixative. The remaining vitreous was carefully removed and the eyecups were 
placed in vials of $2.5 \%$ glutaraldehyde fixative. The optic nerve was removed from the eyecup and placed in the same fixative. Tissues were fixed at room temperature for at least 24 hours, with gentle agitation and stored in fixative at $4^{\circ} \mathrm{C}$.

\subsubsection{Histology and Morphological Analysis}

Sections from the midline of fixed retinas were dissected out and prepared for light and electron microscopy. Dissected areas, shown in Figure 2-1, included areas from the central retina (A1, A2, D1, D2), superior mid-peripheral retina (B1, B2), inferior mid-peripheral retina (E1, E2) and the ora serrata $(C 1, C 2)$. The red lines in Figure 2-1 indicate the face from which sections were taken.

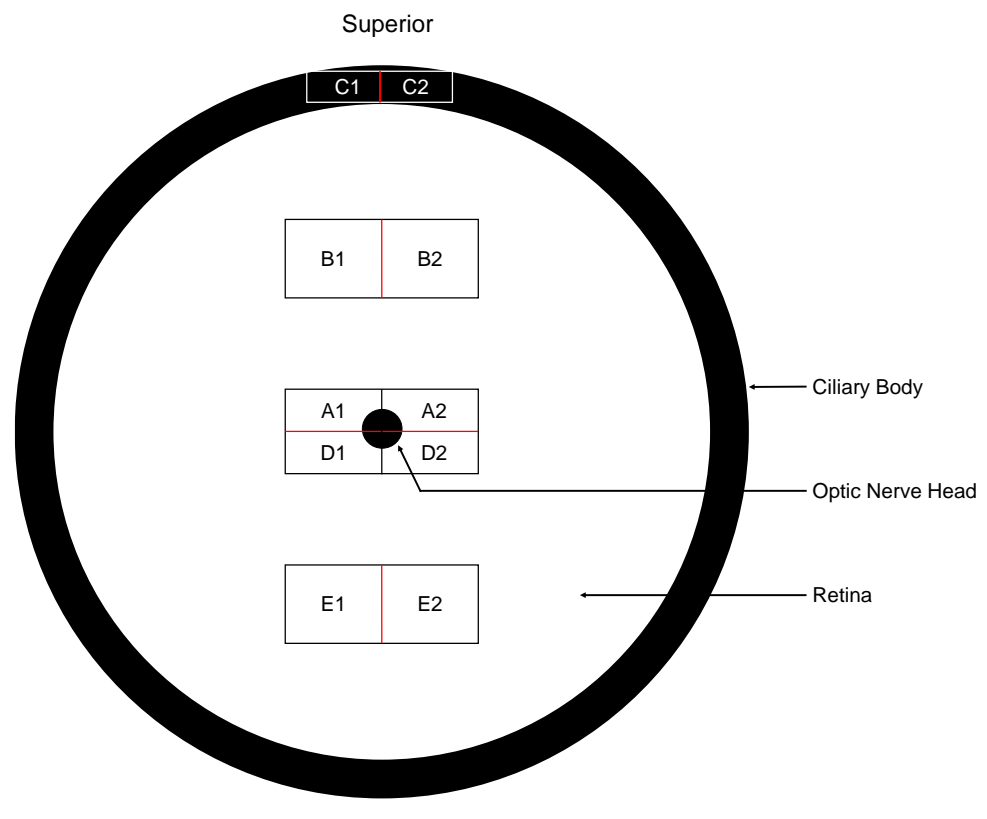

Inferior

Figure 2-1: Diagram of retinal dissection

Samples were washed with $0.1 \mathrm{M}$ sodium cacodylate/0.13 M sucrose/0.01 M 2-mercaptoehtanol buffer ( $\mathrm{pH} 7.4)$ and ultrapure water, post-fixed at $4^{\circ} \mathrm{C}$ with 
$1 \%$ osmium tetroxide, dehydrated in an acetone gradient and infiltrated in increasing concentrations of soft Epon resin (EMS, Hatfield, PA) in acetone followed by exchanges of pure resin. Samples were embedded in resin and polymerized at $60^{\circ} \mathrm{C}$ for 24 hours.

For light microscopy, $0.8 \mu \mathrm{m}$ thick cross-sections of the retina and ciliary body from each area and cross-sections of the optic nerves were cut on a Reichert Ultracut S utramicrotome (Leica Microsystems Inc., Buffalo Grove, IL) with a glass knife. Sections were mounted on glass slides, stained with toluidine blue and coverslipped with Permount mounting solution.

Images of regions of the central retina (A1), superior mid-peripheral retina (B1) and inferior mid-peripheral retina (E1) were obtained with a Leica AF6000 microscope and 40X objective and cropped to $400 \mu \mathrm{m}$. Nuclei in the inner and outer nuclear layers were manually counted using Photoshop and ImageJ (https://imagej.nih.gov/ij/) software along the horizontal mid-line of the eye within $500 \mu \mathrm{m}$ of the optic nerve (central) or along the superior-inferior midline halfway between the optic nerve head and the superior (superior) or inferior (inferior) ora serrata. Images were opened in Photoshop and individual nuclei were marked on a separate image layer which was then exported to ImageJ for automated counting of the marks with the 'Analyze Particles' function.

For late start dogs, a $0.25 \mu \mathrm{m}$ thick cross section of the optic nerve was prepared for light microscopy in the same manner as retinal samples. Images of the entire cross section of the optic nerves were captured with the programmed 
stage of the Leica AF6000 microscope and Leica Application Suite X software. Images were captured with a 40X objective and stitched into a composite image. Composite images were opened in Adobe Photoshop Touch (Adobe Systems, Inc., San Jose, CA) on an iPad (Apple Inc., Cupertino, CA) and counted manually by placing a mark over each axon in a separate image layer. This layer was exported to ImageJ and the marks were counted with the 'Analyze Particles' function.

Samples from these blocks were also prepared for transmission electron microscopy (TEM). 70 to $80 \mathrm{~nm}$ thick ultrathin sections were cut on a Reichert Ultracut S utramicrotome with a diamond knife (Diatome USA, Hatfield, PA) and collected on 200 mesh thin-bar copper grids (EMS, Hatfield, PA). Grids were stained with Sato's triple lead stain and $5 \%$ aqueous uranyl acetate and examined with a JEOL1400 transmission electron microscope (JEOL USA, Inc., Dearborn, MA) using an accelerating voltage of $80 \mathrm{kV}$. Images were acquired using a Gatan digital camera (Gatan, Inc., Pleasanton, CA). Electron micrographs were used to quantify the amount of storage material in a minimum of ten retinal ganglion cells in the central retina (area A1) of late start dogs. Images were opened in Adobe Photoshop and the lasso tool was used to outline the cell body, nucleus and storage bodies of retinal ganglion cells. The area of each structure was used to calculate the percent of the cytoplasm containing storage bodies. 


\subsubsection{Determination of rhTPP1 Concentration}

rhTPP1 concentration was analyzed in vitreous, aqueous and plasma of late start dogs. Frozen vitreous and aqueous humor samples were shipped on dry ice to BioAgilytix Labs (Durham, NC) where analyses were performed with a custom electrochemiluminescence enzyme-linked immunosorbent assay (ELISA) using a monoclonal mouse anti-TPP1 capture antibody prepared and characterized by BioMarin Pharmaceutical and a Sulfo-Tag- (ruthenium) labeled anti-TPP1 detection antibody. Standard curves were generated by spiking purified TPP1 into aqueous and vitreous samples and concentrations of rhTPP1 in study samples were determined by comparing the ruthenium-specific absorbance to that of the standard curves.

Whole blood was periodically collected from the jugular or a peripheral vein into vacutainer tubes containing $\mathrm{K}_{2}$ EDTA as an anticoagulant. Blood samples were centrifuged for 10 minutes at $1591 \mathrm{xg}$ (Unico Powerspin centrifuge, "Blood" preset) and plasma was collected and stored at $-80^{\circ} \mathrm{C}$. The plasma samples were shipped on dry ice to Eurofins Pharma Bioanalytics Services US Inc. (St. Charles, MO) for determination of plasma rhTPP1 concentrations. Analyses were performed using an ELISA that employed the same anti-TPP1 detection antibody as was used for the vitreous and aqueous humor assays. 96-well flat bottom plates were coated with $100 \mu \mathrm{L}$ per well of 1 $\mu \mathrm{g} / \mathrm{mL}$ mouse monoclonal anti-TPP1 antibody clone 254008 (Research and Diagnostic Systems, Inc., Minneapolis, MN, catalogue \# MAB2237) diluted in 0.2 M sodium carbonate-bicarbonate buffer ( $\mathrm{pH} 9.4)$, sealed, and incubated 
overnight at $2-8^{\circ} \mathrm{C}$, static. The next day, plates were washed 3 times with $0.1 \%$ Tween-20 in 1X phosphate buffered saline (PBS) wash buffer. Plates were blocked with casein in 1X PBS (Thermo Scientific, Waltham, MA), sealed and incubated for 1-2 hours at room temperature, static, then washed. The standard curve $(7.8125-2,000 \mathrm{pg} / \mathrm{mL})$ was prepared by diluting rhTPP1 in $5 \%$ matrix diluent ( $5 \%$ canine $\mathrm{K}_{2}$ EDTA plasma in assay diluent, $0.05 \%$ Tween-20 in casein in 1X PBS). Quality controls were prepared by diluting rhTPP1 in canine $K_{2} E D T A$ plasma. Samples and controls were diluted 1:20 in assay diluent prior to addition to the plate. $100 \mu \mathrm{L}$ per well of standards, quality controls and study samples were added. Plates were sealed, incubated for 1-2 hours at room temperature while shaking, then washed. One hundred $\mu \mathrm{L}$ of affinity-purified polyclonal rabbit anti-rhTPP1 detection antibody was added to each well, then plates were incubated for 1-2 hours at room temperature while shaking, then washed. One hundred $\mu \mathrm{L}$ per well of goat anti-rabbit lgG $(\mathrm{Fc})$-horseradish peroxidase conjugated antibody (Jackson ImmunoResearch Laboratories, Inc., West Grove, PA, catalogue \# 111-035-046) was added and plates were incubated for 1 hour at room temperature while shaking, then washed. One hundred $\mu \mathrm{L}$ of tetramethylbenzidine peroxide substrate solution (Thermo Scientific, Waltham, MA) was added to each well and the plates were incubated for 10-15 minutes protected from light at room temperature while shaking. The reaction was stopped with the addition of $100 \mu \mathrm{L} 2 \mathrm{~N}$ sulfuric acid $\left(\mathrm{H}_{2} \mathrm{SO}_{4}\right)$ and the optical density signal was read at $450 \mathrm{~nm}$. 


\subsubsection{Identification of anti-TPP1 Antibodies}

Frozen $\mathrm{K}_{2}$ EDTA blood plasma samples collected from late start dogs as described above were shipped on dry ice to ICON Laboratory Services, Inc. (Whitesboro, NY) for determination of the plasma titer of anti-TPP1 antibodies using an electrochemiluminescence assay. Streptavidin QUICKPLEX 96-well plates were blocked with $150 \mu \mathrm{L}$ per well of $1 \%$ MSD Blocker A in 1X PBS (Meso Scale Discovery, Rockville, MD). $50 \mu \mathrm{L}$ of study samples, positive control calibrators consisting of an anti-TPP1 antibody in canine plasma, and a pooled canine plasma negative control were diluted 1:10 in Diluent Buffer (1\% MSD Blocker A, $0.05 \%$ Tween in 1X PBS) and loaded into the wells of a polypropylene transfer plate. The samples were then diluted $1: 3$ by the addition of $100 \mu \mathrm{L}$ per well of a 3X Master Mix containing $0.250 \mu \mathrm{g} / \mathrm{mL}$ each of ruthenylated (Sulfotagged) TPP1 and biotinylated TPP1 labels in diluent buffer resulting in a final plasma concentration of $3.33 \%$. Plates were incubated at room temperature for one hour protected from light with shaking then washed with $0.05 \%$ Tween in $1 \mathrm{X}$ PBS. $50 \mu \mathrm{L}$ from each well was transferred to the blocked streptavidin-coated plate and incubated at room temperature for 1 hour protected from light with shaking. Plates were washed again and a tripropylamine-containing read buffer (2X MSD Read Buffer T with surfactant) was added. Plates were read within 1 minute on an MSD QuickPlex SQ 120 plate reader. The chemiluminescent signal produced by ruthenium in the presence of tripropylamine upon application of voltage is proportional to the amount of TPP1 antibody present. The mean signal was compared to the assay cut point determined during assay validation to 
determine whether a sample was positive or negative. End point titer analysis was performed on positive samples. For this, 1:10 diluted samples were titered 4fold into $10 \%$ canine plasma. The last dilution above the titer cut point was reported as the reciprocal of the dilution point.

\subsubsection{Statistical Analysis}

All statistical tests were performed using SigmaPlot (Systat Software Inc., San Jose, CA). Data were subjected to the Shapiro-Wilk test to confirm normal distribution. For early start dogs E1-E4, a repeated measures 2-way ANOVA was used to compare ERG b-wave amplitudes and b:a-wave amplitude ratios from CLN2-affected eyes treated with intravitreal rhTPP1 to those from vehicle-treated eyes. Follow-up pairwise comparisons were performed with the Holm-Sidak correction $(\alpha=0.05)$ to control family-wise error rate. Because each of the late start dogs was subjected to a different treatment regimen, ERG data from the 4 dogs were not pooled for statistical analysis. For early start dogs, statistical comparison of the retinal cell counts between the rhTPP1- and vehicle-treated eyes was performed separately for each retinal region using a Student's t-test. The retinal cell count data for both the inner and outer nuclear layers of late start dogs were not normally distributed, so comparisons between the rhTPP1- and vehicle-treated eyes were performed using the Mann-Whitney Rank Sum Test. Optic nerve axon count data were normally distributed, so the comparison between rhTPP1- and vehicle-treated eyes was performed using a Student's ttest. 


\subsection{Results}

2.3.1 rhTPP1 Concentration in Aqueous Humor, Vitreous Humor and Plasma

None of the vehicle-treated eyes had detectable levels of rhTPP1 in the aqueous humor. Measurable amounts of rhTPP1 were present in the aqueous humor of the rhTPP1-treated eyes of 3 of the 4 late start dogs (Table 2-3). A substantial amount of rhTPP1 was present in the aqueous of dog L1 that was euthanized one day after the last IVT injection. In rhTPP1-treated eyes injected at longer intervals before euthanasia and sample collection, the aqueous levels of rhTPP1 were near or below the limit of quantitation.

None of the vehicle-treated eyes had detectable levels of rhTPP1 in the vitreous humor with the exception of dog L1 in which the control eye had 14.6 $\mathrm{ng} / \mathrm{mL}$ of rhTPP1. At the same time the rhTPP1 concentration in the vitreous of the rhTPP1-treated eye was $62,365 \mathrm{ng} / \mathrm{mL}$ (Table 2-3). This indicates that a very small amount of rhTPP1 may have traveled from the rhTPP1-treated eye to the contralateral eye shortly after injection. None of the other treated eyes had detectable levels of rhTPP1 in the vitreous at the time points analyzed.

\begin{tabular}{|c|c|c|c|}
\hline Dog & $\begin{array}{c}\text { Aqueous TPP1 } \\
\text { Conc (ng/mL) }\end{array}$ & $\begin{array}{c}\text { Vitreous TPP1 } \\
\text { Conc (ng/mL) }\end{array}$ & $\begin{array}{c}\text { Days Since Last } \\
\text { Injection }\end{array}$ \\
\hline L1 & 15754 & 62365 & 1 \\
\hline L2 & $B L{ }^{*}$ & BLQ & 67 \\
\hline L3 & 9.23 & $B L Q$ & 140 \\
\hline L4 & 4.48 & $B L Q$ & 48 \\
\hline
\end{tabular}

${ }^{*} \mathrm{BLQ}$ - below limit of quantitation;

limit of quantitation = $3.91 \mathrm{ng} / \mathrm{mL}$ for aqueous, $1.95 \mathrm{ng} / \mathrm{mL}$ for vitreous

Table 2-3: Concentrations of rhTPP1 in the aqueous and vitreous of rhTPP1treated eyes 
Plasma rhTPP1 concentrations were determined in samples obtained at times ranging from 1 to 140 days after the first or second IVT injections. In none of the samples was the plasma concentration above the limit of quantitation (1.25 $\mathrm{ng} / \mathrm{mL}$ ). In an additional affected dog, plasma samples were analyzed for rhTPP1 concentrations at 1, 3, 6, 24, 48 and 96 hours after an IVT injection of $1 \mathrm{mg}$ rhTPP1. Barely detectable concentrations of rhTPP1 were only observed at the 6, 24 and 48 hour time points, peaking at $3.36 \mathrm{ng} / \mathrm{mL}$ at 24 post-injection.

\subsubsection{Ocular Inflammation}

No dogs developed intraocular inflammation following the first injection of rhTPP1 and at no time was intraocular inflammation observed in the control eyes that received vehicle injections. No adverse systemic effects of the treatments were observed clinically or detected with periodic complete blood counts and blood chemistry analyses.

All early start dogs exhibited intraocular inflammation to varying degrees following each injection after the first, typically characterized by transient anterior and posterior uveitis that was mitigated by sub-Tenon's injection of triamcinolone at the time of rhTPP1 administration and controlled with topical anti-inflammatory medications. While active inflammation resolved over time, areas of vitreal opacity persisted long-term and were apparent with in vivo imaging (Figure 2-2). This inflammatory response prompted a reduction in dosing frequency for dogs

E2 and E3, from every 2 to every 3 weeks (see Table 2-1). More severe inflammatory reactions occurred approximately one week after administration of 
a $0.3 \mathrm{mg}$ dose in dog E1 and after the second dose in dog E4. These reactions were characterized by anterior and posterior uveitis, localized retinal hemorrhage, and inflammation of the optic nerve head. In dog E1, the inflammation persisted for several weeks and resulted in a marked reduction in ERG amplitudes (Figure 2-5). Based on these symptoms, the next scheduled injection was delayed to 6 weeks later and the dose was reduced to $0.1 \mathrm{mg}$. Clinical signs of inflammation largely resolved within one month after this injection, but dog E1 received no further intravitreal rhTPP1 injections. For dog $\mathrm{E} 4$, in order to allow the treatment-related inflammation to resolve completely, the dosing interval was increased to 6 weeks and this interval was used for the remainder of the study. This allowed inflammation to fully resolve between doses and minimized long term vitreal opacity. 


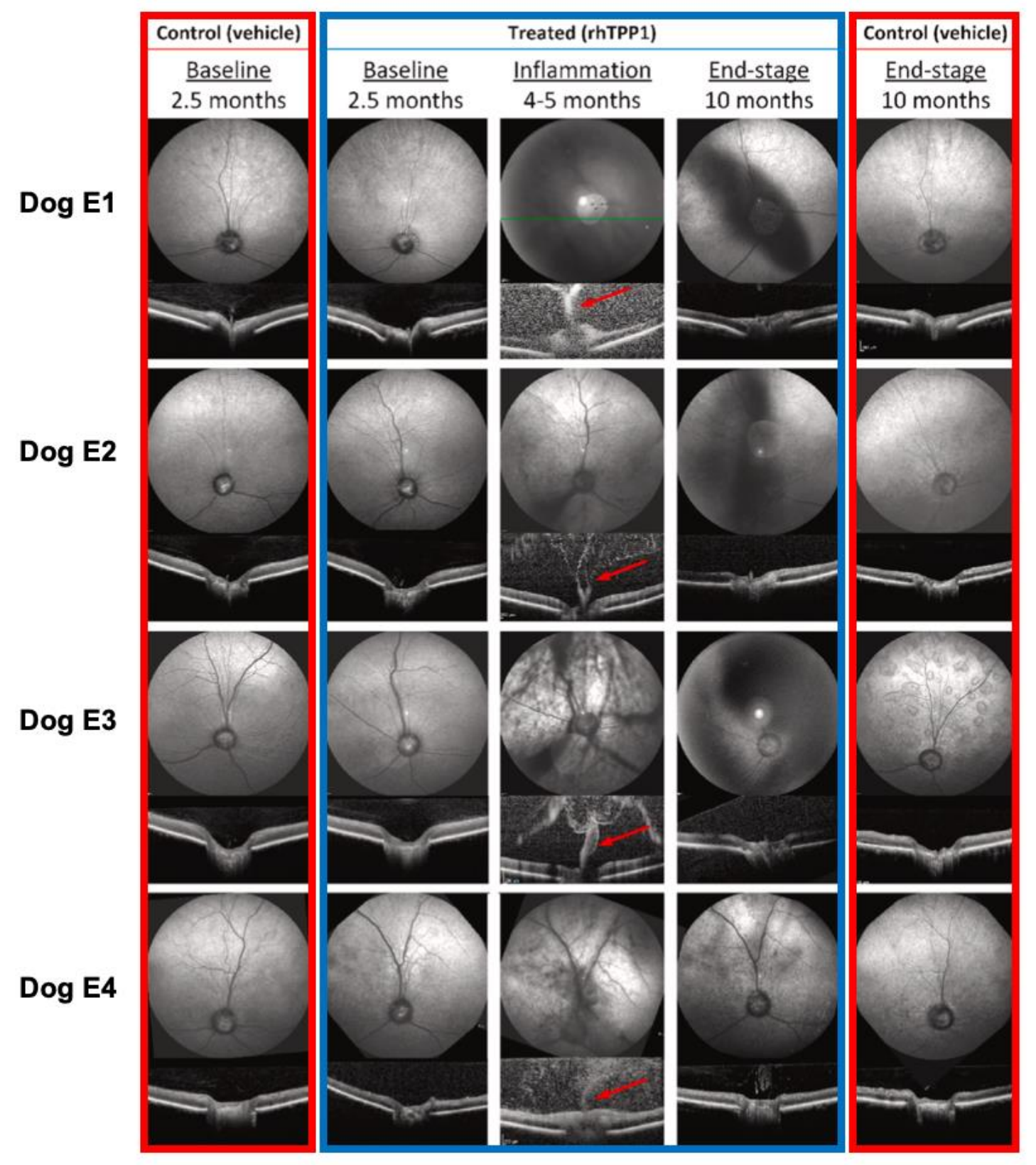

Figure 2-2: Scanning laser ophthalmoscopy (SLO) fundus images from all early start dogs throughout the study. Inflammation that occurred early in the study resulted in long term vitreal opacity in treated eyes despite the resolution of active inflammation. By 10 months of age, dog E3 had developed widespread disease-related canine multifocal retinopathy lesions in the control eye but not in the treated eye. 
Late start dogs received higher $(0.5$ and $0.25 \mathrm{mg}$ ) but less frequent doses of rhTPP1. Dogs L2 and L4 exhibited mild intraocular inflammation characterized by transient anterior uveitis and vitritis the day after the second dose, and in both cases the active inflammation had resolved by 3-4 weeks post injection. However, vitreal opacity persisted through the remainder of the study in both dogs, though to a greater degree in dog L2 than in dog L4 (Figure 2-3). Dog L3 received only one dose and dog $L 1$ required euthanasia due to a neurological event unrelated to therapeutic dosing the day after the second dose and therefore neither exhibited inflammation. 


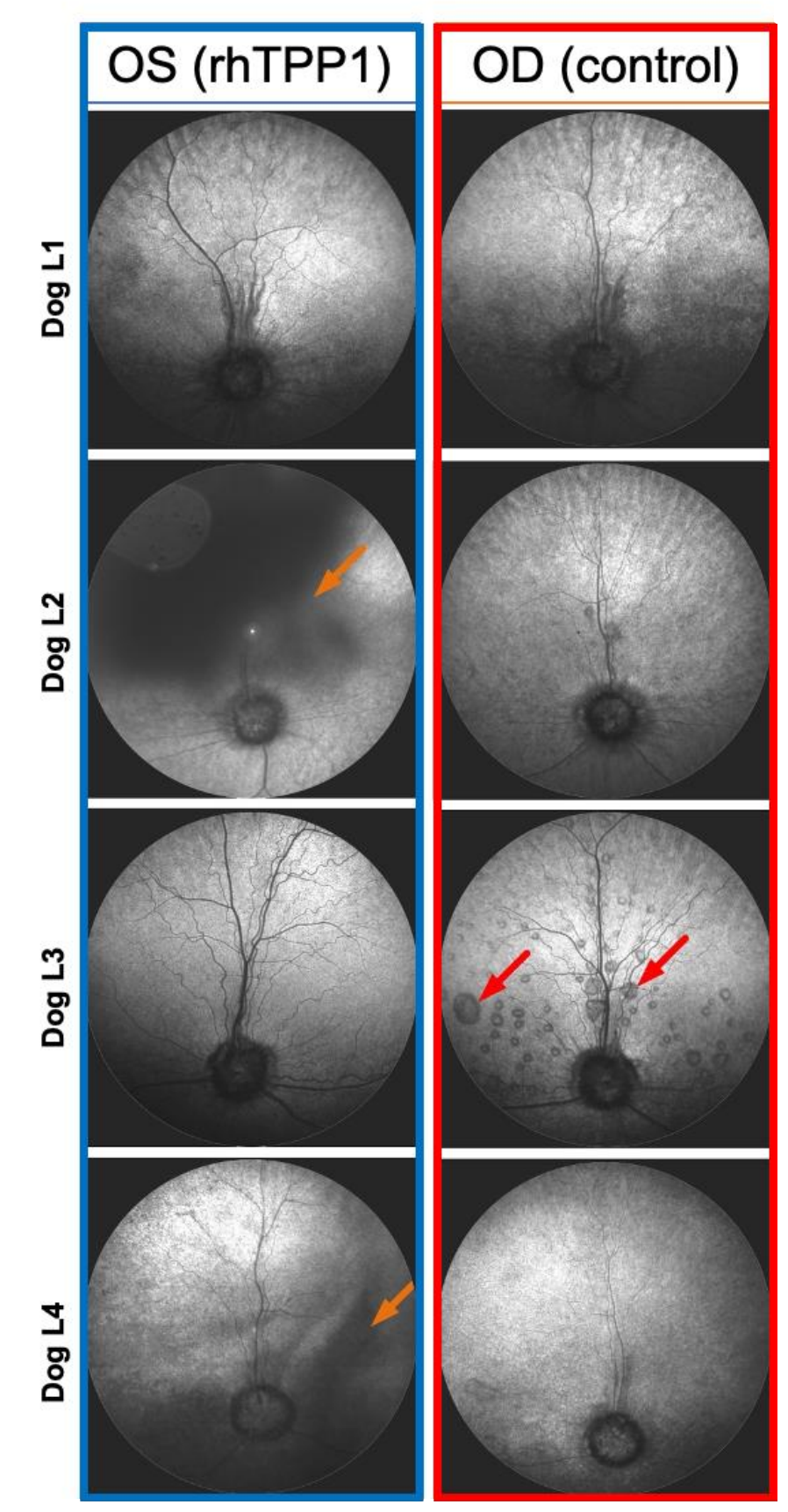

Figure 2-3: SLO fundus images from all late start dogs at the end of the study. Images remained normal in all dogs following the initial dose, but dogs L2 and L4 exhibited intraocular inflammation following the second dose. While active inflammation gradually resolved, vitreal opacity persisted through the remainder of the study in both dogs (orange arrows). Final images for dog L1 were taken days prior to the second dose as follow-up images were not possible due to unscheduled euthanasia. By 10 months of age, dog L3 had developed widespread disease-related CMR lesions in the control eye (red arrows) but not in the treated eye. 
Intraocular inflammation was microscopically characterized by perivascular to interstitial infiltrate of predominantly lymphocytes and plasma cells in the ciliary body and retina. Inflammatory cells were only observed in the rhTPP1-treated eyes, suggesting that this perivascular cuffing was the result of an immune reaction to the rhTPP1. The inflammatory reaction was characterized in detail in the eyes of late start dogs. Perivascular cuffing was observed around blood vessels in the retinal ganglion cell/nerve fiber layer in some of the rhTPP1treated eyes of these dogs (Figure 2-4). When present, the degree of perivascular cuffing was more pronounced in the mid-peripheral retina than in the central retina. The degree of perivascular cuffing correlated with the time between the last rhTPP1 injection and euthanasia (Table 2-4). No cuffing was present in the treated retina of dog $L 3$ who received a single injection of $0.5 \mathrm{mg}$ of rhTPP1 at 25 weeks of age and was euthanized at 44.5 weeks of age. Likewise, perivascular cuffing was not observed in the treated eye of dog L1, who also received an injection of $0.5 \mathrm{mg}$ of rhTPP1 at 25 weeks and then a second injection of $0.25 \mathrm{mg}$ of rhTPP1 just prior to euthanasia at 40 weeks of age. On the other hand, both dogs L2 and L4 exhibited significant perivascular cuffing around the inner retinal vessels of the rhTPP1-treated eyes, with the cuffing being more pronounced in dog L2. Both dogs were treated with $0.5 \mathrm{mg}$ rhTPP1 at 25 weeks of age. Dog L4 received a subsequent injection of $0.25 \mathrm{mg}$ rhTPP1 at 36 weeks of age and was euthanized 4 weeks later, and dog L2 received an injection of $0.25 \mathrm{mg}$ rhTPP1 at 33.5 weeks of age and was euthanized one week later. These data suggest that the inflammation indicated by the perivascular 
cuffing around inner retinal vessels subsided over time after administration of rhTPP1. This is consistent with the clinical observations of inflammation noted in the ophthalmic examinations of these dogs.

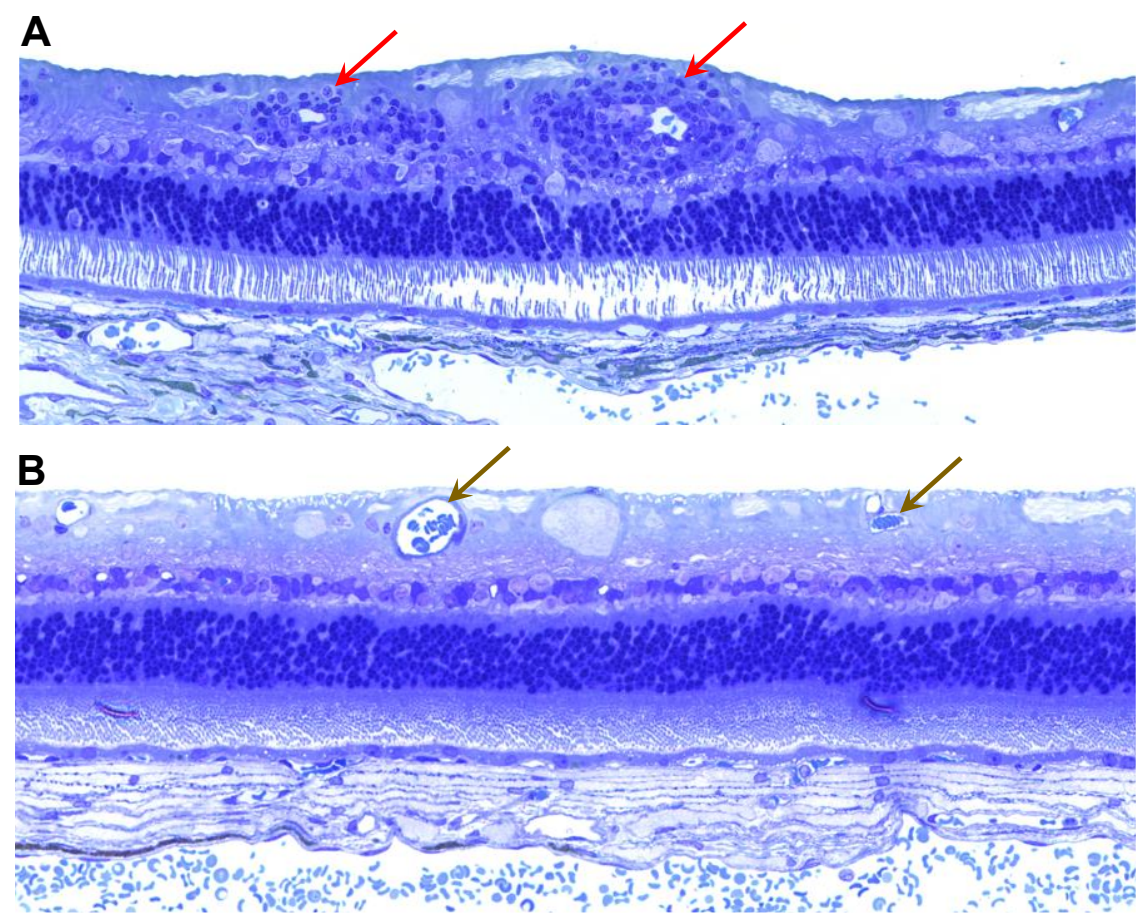

Figure 2-4: Light micrographs of sections of the mid-peripheral rhTPP1-treated retinas from dogs $L 2(A)$ and $L 3(B)$. Pronounced perivascular cuffing was present around inner retinal vessels in the central retina of dog L2 (red arrows), whereas no such cuffing was present around inner retinal vessels (brown arrows) of the retina in dog L3.

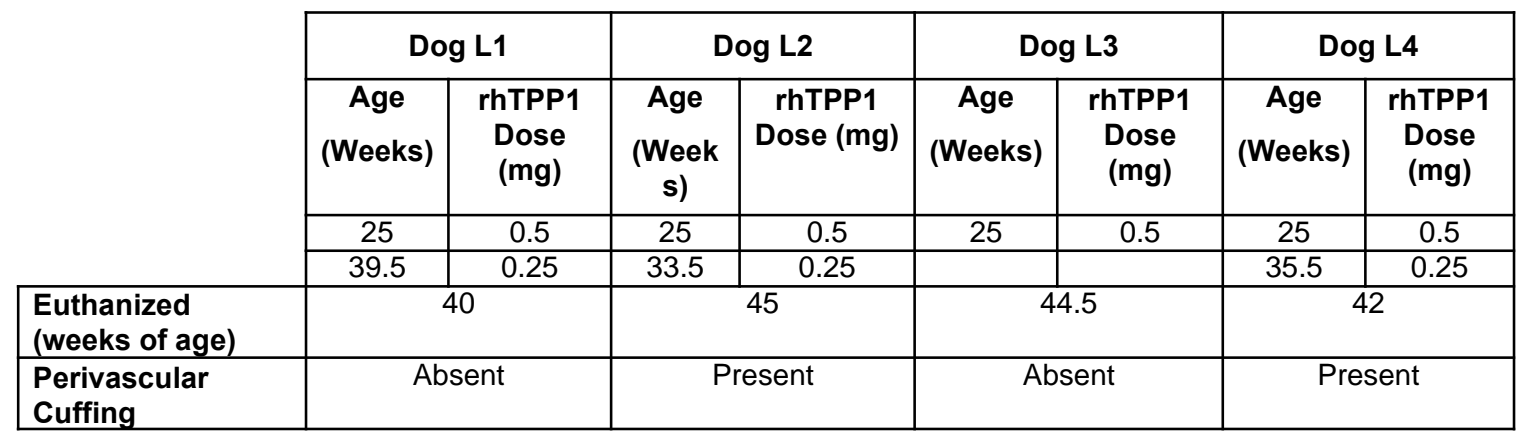

Table 2-4: Perivascular cuffing around inner retina vessels 


\subsubsection{Plasma anti-TPP1 Antibody Titers}

Intravitreal injection of rhTPP1 resulted in a robust systemic immune response to TPP1 as indicated by the presence of anti-TPP1 antibodies in the blood plasma of the treated dogs. In plasma samples obtained prior to the first rhTPP1 injection, no anti-TPP1 antibodies could be detected in any of the dogs.

Dog L3, which received a single injection of $0.5 \mathrm{mg}$ rhTPP1 at 25 weeks of age, had a modest antibody titer at 140 days post-injection (Table 2-5). The remaining dogs received two IVT injections of rhTPP1, $0.5 \mathrm{mg}$ at 23.5 to 25 weeks of age and $0.25 \mathrm{mg}$ at 33.5 to 39.5 weeks of age (Table 2-2). At 67 and 48 weeks after the second injections, dogs L2 and L4 had very high antibody titers (Table 2-5).

Dog L1 had to be euthanized one day after the second IVT injection. At this time, 140 days after the first IVT injection, the antibody titer was minimal (Table 2-5), suggesting that the dog had not yet mounted a full immune response to the second injection.

\begin{tabular}{|c|c|c|}
\hline Dog & $\begin{array}{c}\text { Anti-TPP1 } \\
\text { Antibody Titer }\end{array}$ & $\begin{array}{c}\text { Days Since Last } \\
\text { Injection }\end{array}$ \\
\hline L1 & 10 & 1 \\
\hline L2 & $>163840^{*}$ & 67 \\
\hline L3 & 640 & 140 \\
\hline L4 & $>163840^{*}$ & 48 \\
\hline
\end{tabular}

*Maximum limit of assay.

Table 2-5: Anti-TPP1 antibody titers in pre-euthanasia blood samples 


\subsubsection{Retinal Function}

All dogs exhibited preservation of retinal function as assessed by electroretinography in the eye treated with intravitreal rhTPP1 relative to the contralateral control eye for all light stimulus conditions (Figures 2-5 to 2-13).

Early start dog E1 exhibited partial preservation of the b-wave after short term treatment (Figure 2-5). In this dog, b-wave amplitudes in the rhTPP1-treated eye were greater than those in the contralateral vehicle-treated eye for all stimulus conditions including pure rod, mixed rod/cone and pure cone responses. There was a marked decrease in all ERG amplitudes in the rhTPP1-treated eye at 4 months of age following a severe inflammatory reaction to the $0.3 \mathrm{mg}$ rhTPP1 dose, but ERG amplitudes recovered once the clinically observable inflammation resolved. Amplitudes gradually decreased toward the end of the study since dog E1 received its final intravitreal injection early in the study period at approximately 5 months of age. 

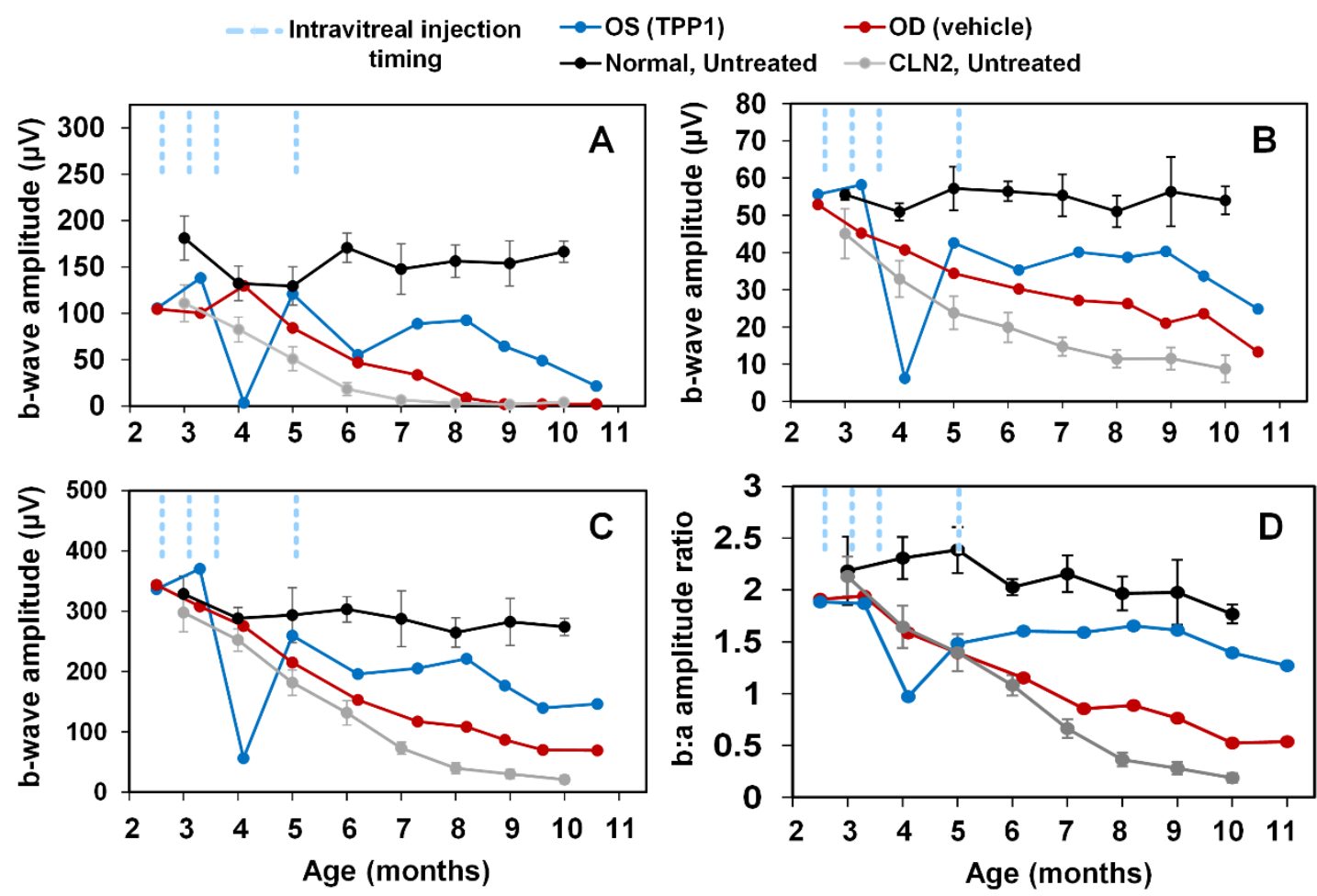

Figure 2-5: Pure rod (A), pure cone (B) and mixed rod/cone (C) ERG response amplitudes and b-wave:a-wave amplitude ratios for the mixed rod/cone response (D) for dog E1. Black and gray traces represent historical data \pm standard error of the mean (SEM) from normal $(n=7)$ and CLN2-affected $(n=6)$ untreated dogs, respectively, from the same research colony.

Sustained and complete preservation of function through 10 months of age was observed in early start dogs E2, E3 and E4 that received long-term treatment courses (Figures 2-6 to 2-8). Significantly greater b-wave amplitudes were observed in the treated eyes of these dogs for pure rod (Figure 2-6), pure cone (Figure 2-7) and mixed rod/cone (Figure 2-8) responses relative to the vehicle-treated eyes. Even at end-stage disease, rod, cone and mixed rod/cone amplitudes in the treated eyes were normal (dogs E3 and E4) or greater than normal (dog E2) while the responses from vehicle control eyes were nonrecordable. Dog E2 exhibited a marked increase in rod (Figures 2-6 and 2-9) and 
mixed rod/cone b-wave amplitudes (Figures 2-8 and 2-9) in the latter half of the study resulting in amplitudes that were greater than the range observed in normal dogs for several of the recordings.
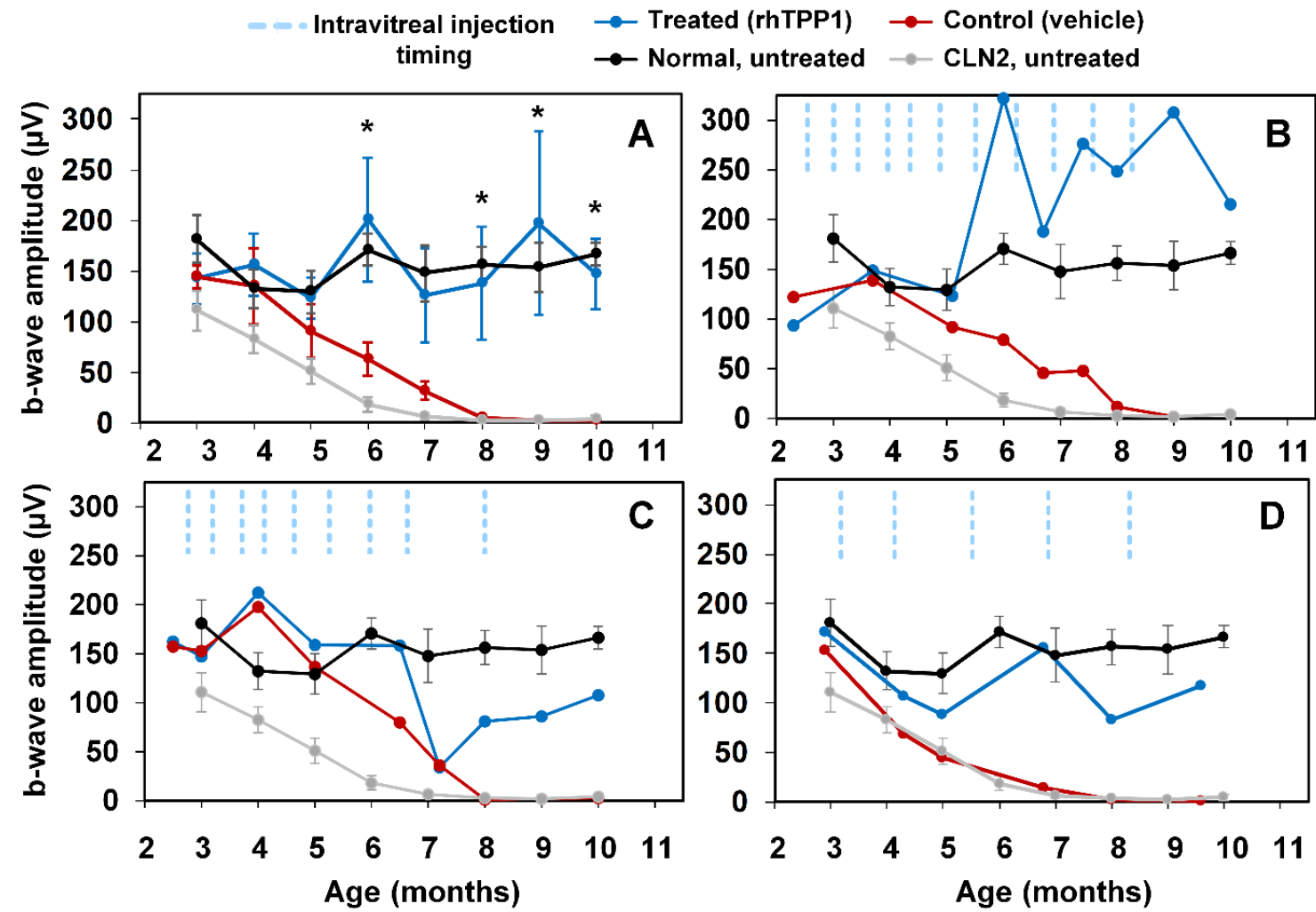

Figure 2-6: Mean \pm SEM (A) and individual (B-D) ERG rod response amplitudes for dogs E2 (B), E3 (C), and E4 (D). Mean rod response b-wave amplitudes in the rhTPP1-treated eyes (OS, blue traces) were greater than in the contralateral vehicle-treated eyes $\left(\mathrm{OD}\right.$, red traces) $\left({ }^{*} p<0.05\right)$. Black and gray traces represent historical data \pm SEM from normal $(n=7)$ and CLN2-affected $(n=6)$ untreated dogs, respectively, from the same research colony. 

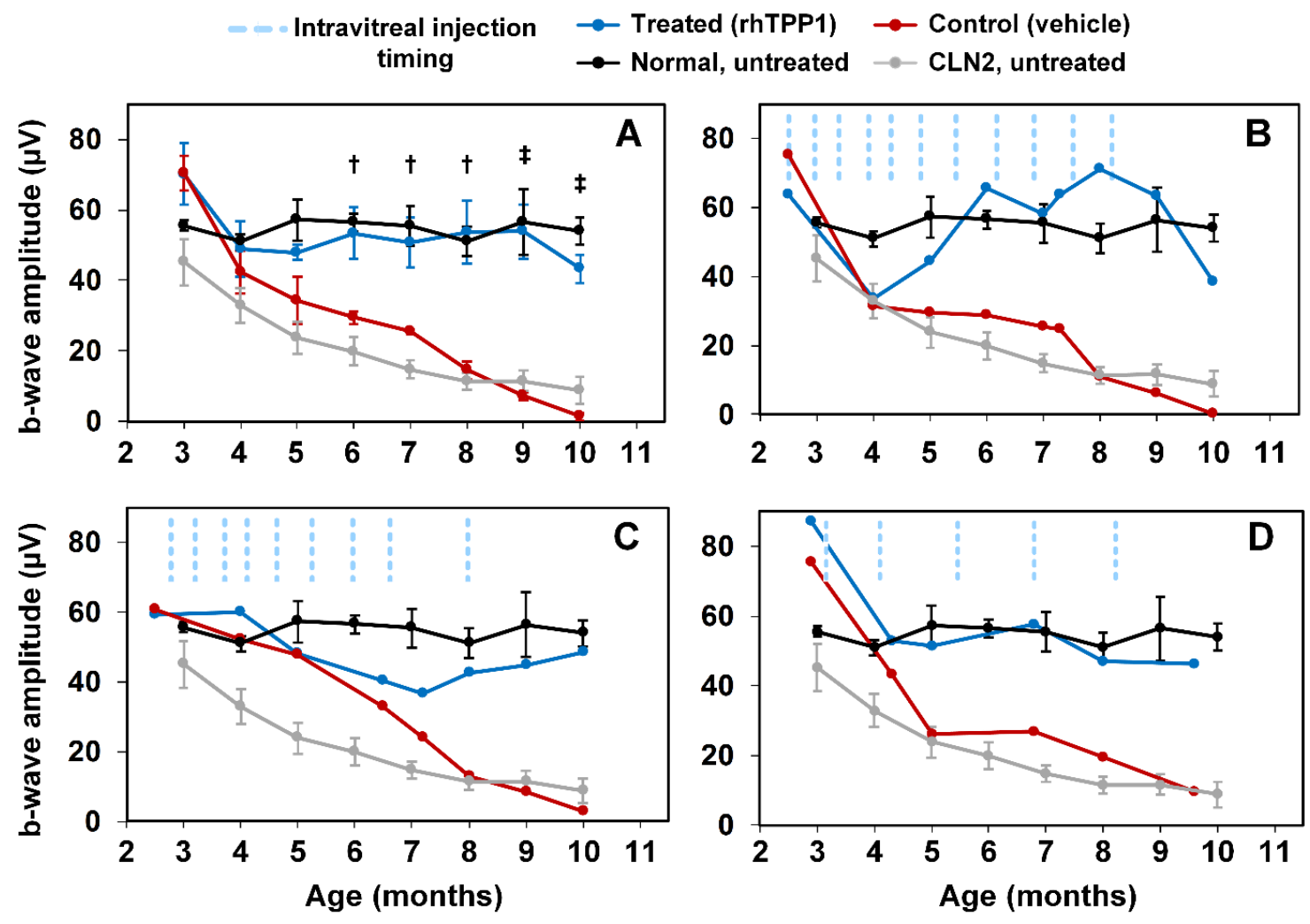

Figure 2-7: Mean \pm SEM $(A)$ and individual (B-D) ERG cone response amplitudes for dogs E2 (B), E3 (C), and E4 (D). Cone response b-wave amplitudes in the rhTPP1-treated eye (OS, blue traces) were greater than the contralateral vehicletreated eye $(\mathrm{OD}$, red traces) $(\dagger p<0.01, \ddagger p<0.001)$. Black and gray traces represent historical data \pm SEM from normal $(n=7)$ and CLN2-affected $(n=6)$ untreated dogs, respectively, from the same research colony. 

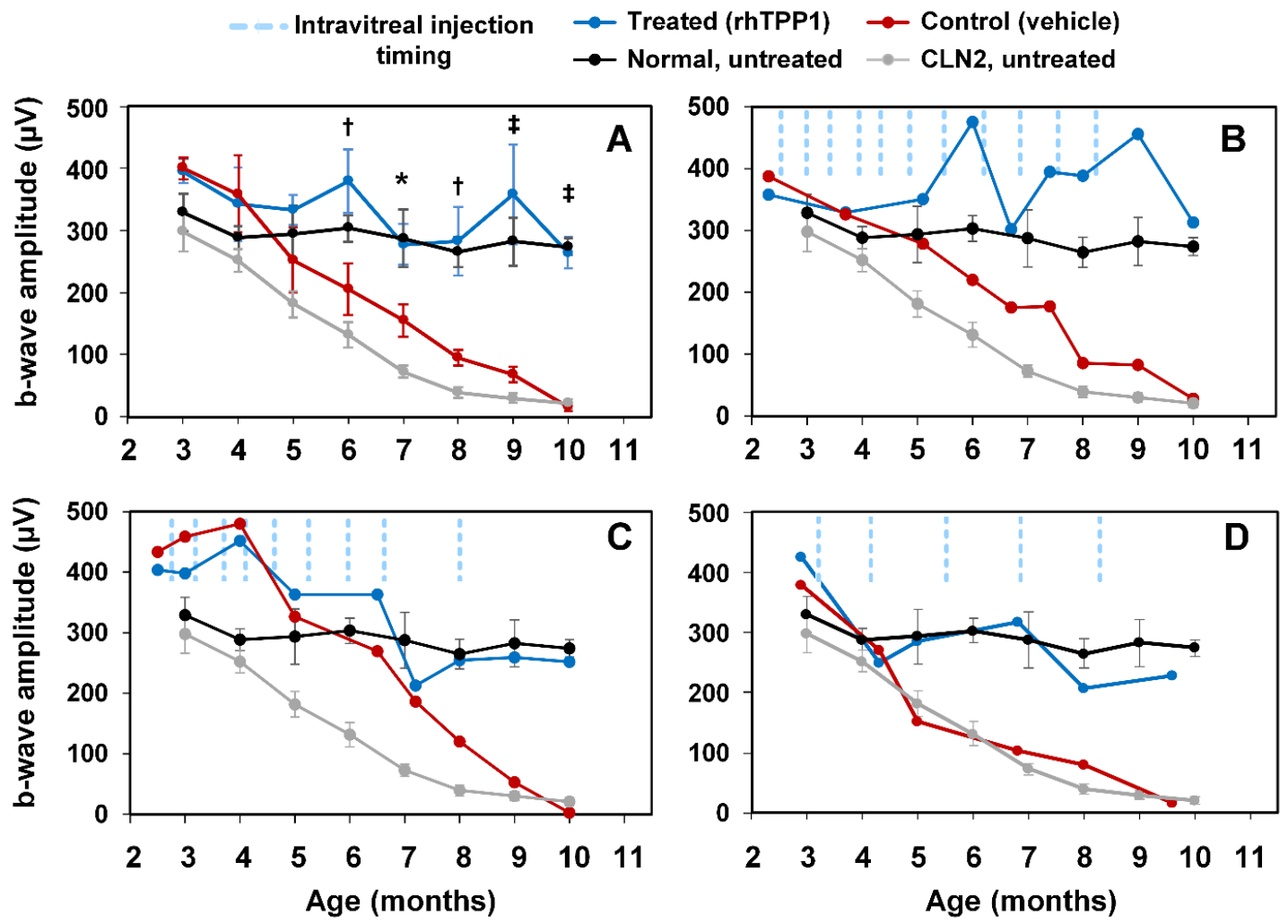

Figure 2-8: Mean \pm SEM (A) and individual (B-D) ERG mixed rod/cone response amplitudes for dogs E2 (B), E3 (C), and E4 (D). Mean mixed response b-wave amplitudes in the rhTPP1-treated eye (OS, blue traces) were greater than the contralateral vehicle-treated eye (OD, red traces) $\left({ }^{*} p<0.05, \uparrow p<0.01, \pm p<0.001\right)$. Black and gray traces represent historical data \pm SEM from normal $(n=7)$ and CLN2-affected $(n=6)$ untreated dogs, respectively, from the same research colony. 


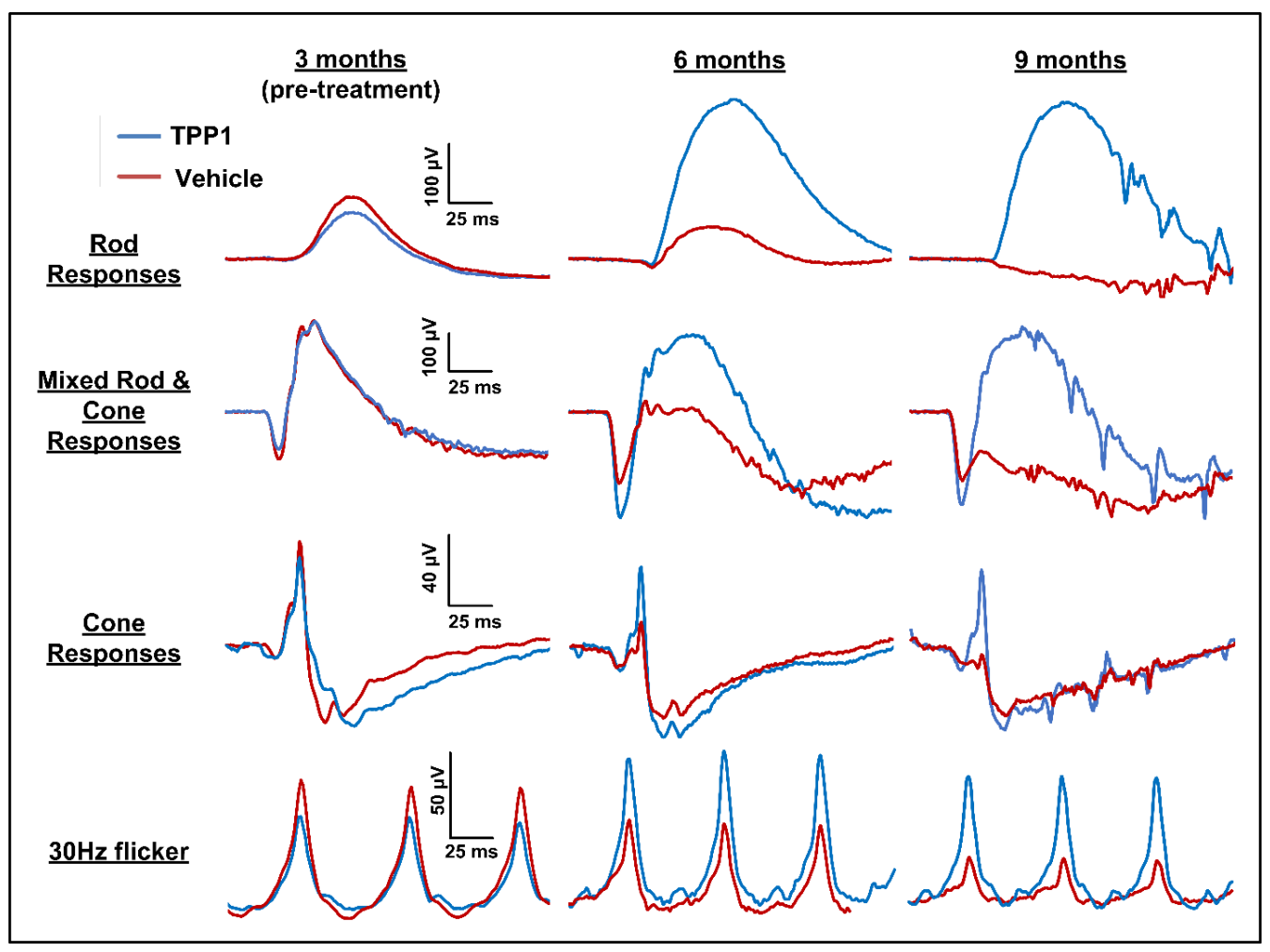

Figure 2-9: ERG waveforms from dog E2 at 3, 6, and 9 months of age.

Responses from the rhTPP1-treated eye (OS, blue traces) remained normal or greater than normal throughout the study period, while the response from the vehicle control eye (OD, red traces) declined dramatically with age, as is typical for untreated CLN2-affected dogs.

Treatment in late start dogs was initiated after reduction of b-wave amplitudes was apparent. Baseline measurements were taken within one week prior to each intravitreal injection of rhTPP1. Following the initial dose of rhTPP1, no significant further declines in mixed rod and cone b-wave amplitudes were observed in any of the late start dogs for at least 12 weeks (Figure 2-12). Dog L1 exhibited a $20 \%$ reduction in mixed rod and cone response b-wave amplitudes 15.5 weeks after the initial dose and a second dose was administered. The day after administration of the second dose, a neurologic episode unrelated to rhTPP1 administration occurred and necessitated humane euthanasia which 
prevented follow-up. Although b-wave amplitudes in Dog L2 remained at baseline \#1 levels, a second dose was administered 8 weeks following the initial dose to investigate the effects of redosing. Following the second dose, b-wave amplitudes remained similar to baseline \#1 measurements for the remainder of the study period. Dogs L3 and L4 exhibited more than $20 \%$ declines in mixed rod and cone b-wave amplitudes 12-13 weeks after the initial dose. Dog L3 was not re-dosed due to the severity of disease-related neurologic symptoms. While bwave amplitudes declined further for the next 2 months, the final ERG measurements were similar to baseline \#1 measurements. Dog L4 exhibited marked intraocular inflammation after the second dose which resulted in significant declines in ERG b-wave amplitudes. However, once the inflammation had resolved, amplitudes returned to the same level as baseline \#2. 

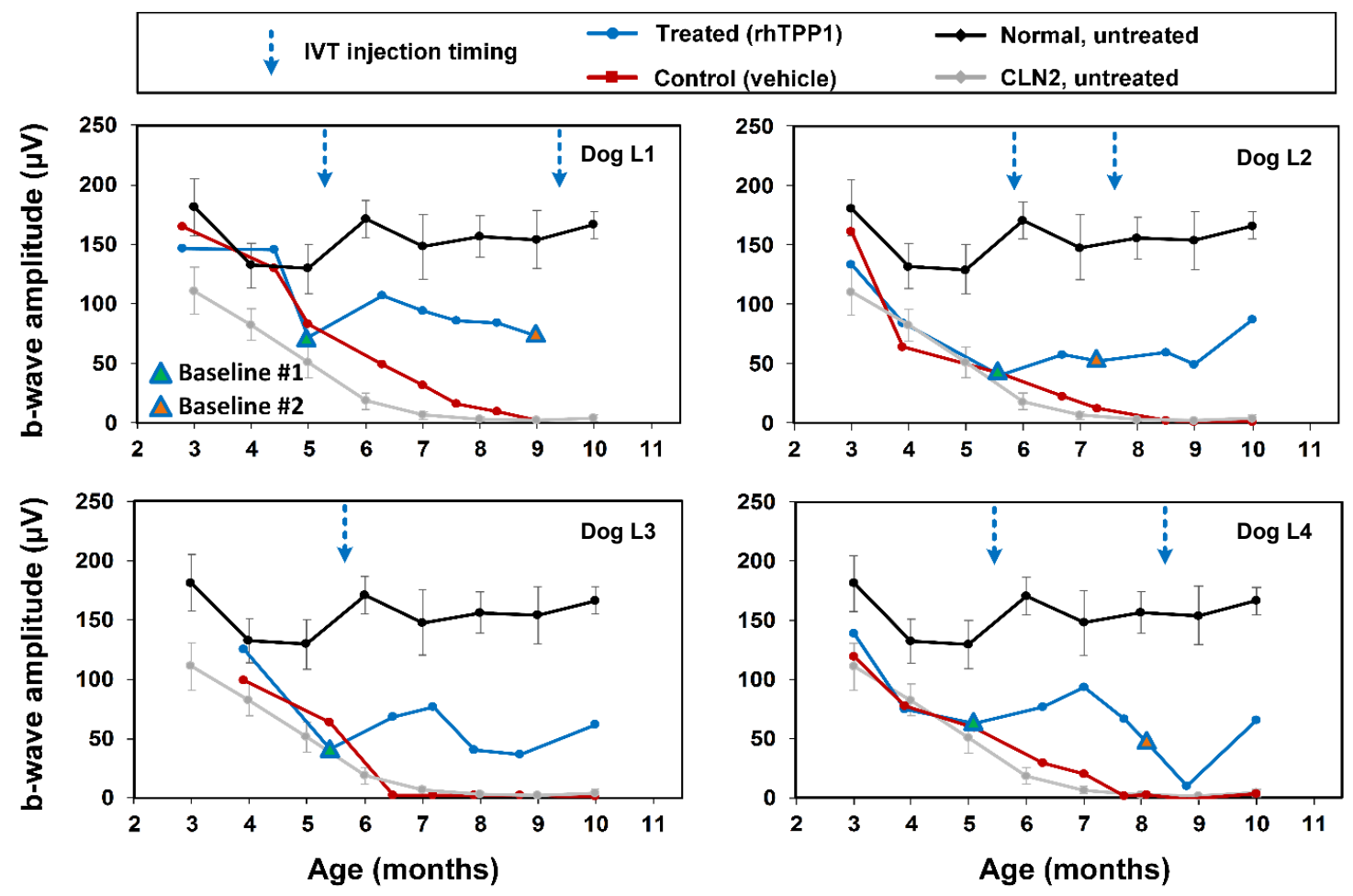

Figure 2-10: Individual rod response amplitudes from dogs L1, L2, L3 and L4. Post symptomatic intravitreal rhTPP1 halted decline of retinal rod responses in CLN2-affected dogs. Intravitreal injections \#1 and \#2 were given within 1 week following the Baseline \#1 and \#2 ERG measurements, respectively. Black and gray traces represent historical data \pm SEM from normal $(n=7)$ and CLN2affected $(n=6)$ untreated dogs, respectively, from the same research colony. 


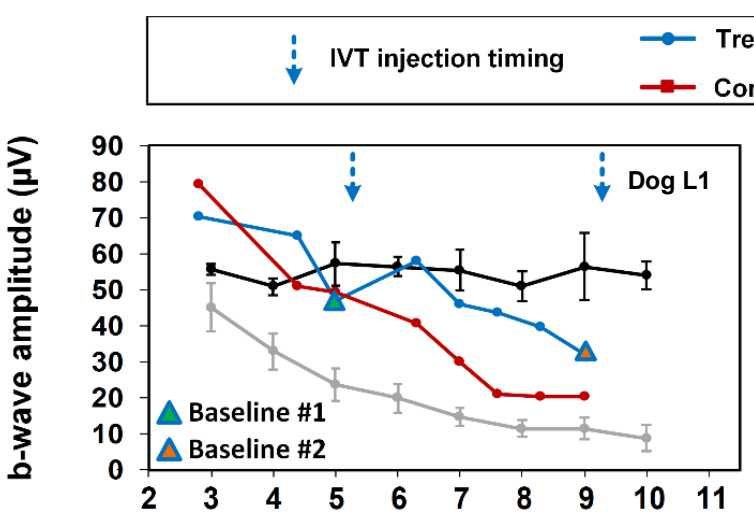

Treated (rhTPP1)
Control (vehicle) $\quad \begin{gathered}\text { Normal, untreated } \\ \text { CLN2, untreated }\end{gathered}$
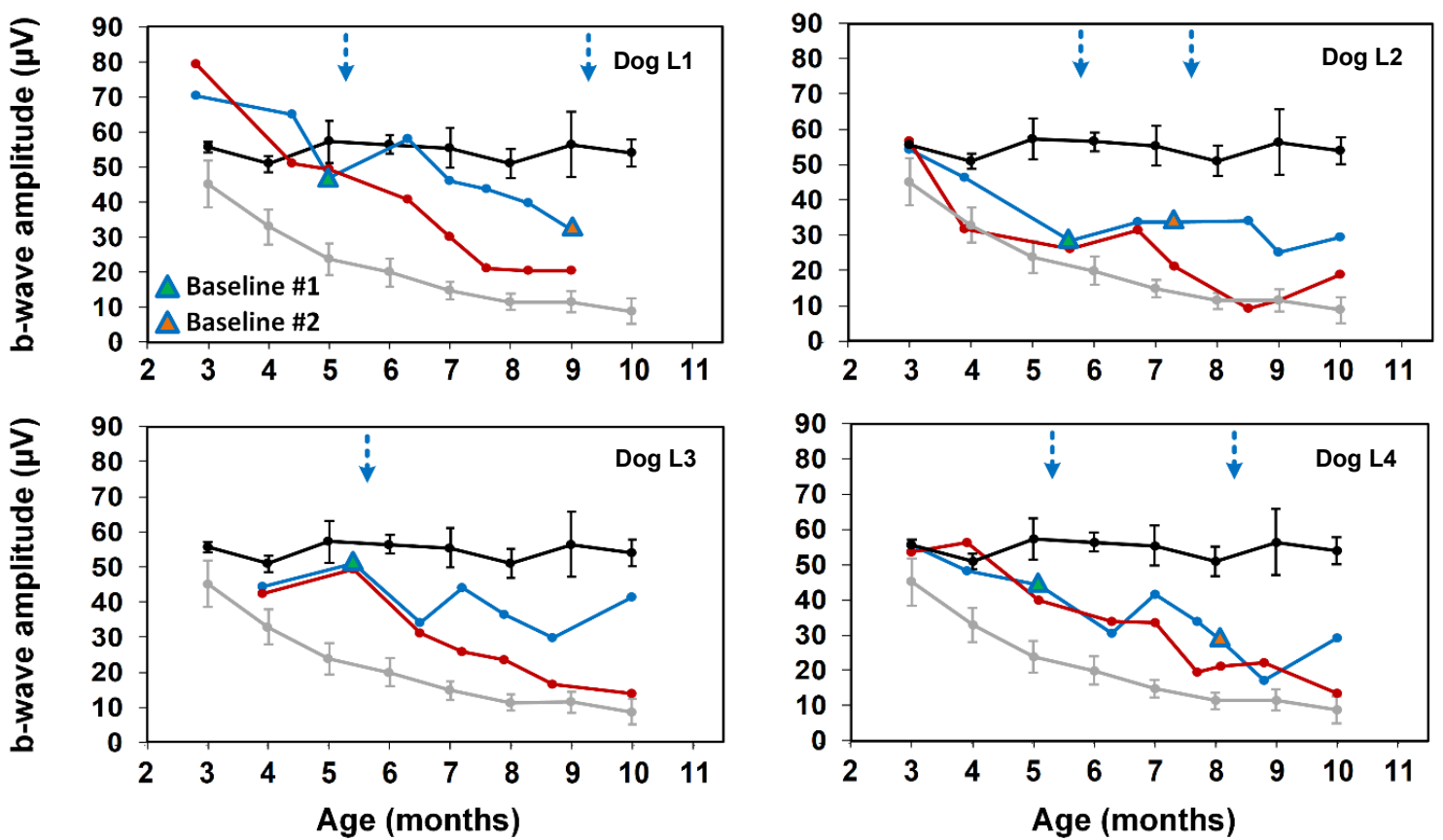

Figure 2-11: Individual cone response amplitudes from dogs L1, L2, L3 and L4. Post symptomatic intravitreal rhTPP1 halted decline of retinal cone responses in CLN2-affected dogs. Black and gray traces represent historical data \pm SEM from normal $(n=7)$ and CLN2-affected $(n=6)$ untreated dogs, respectively, from the same research colony. 

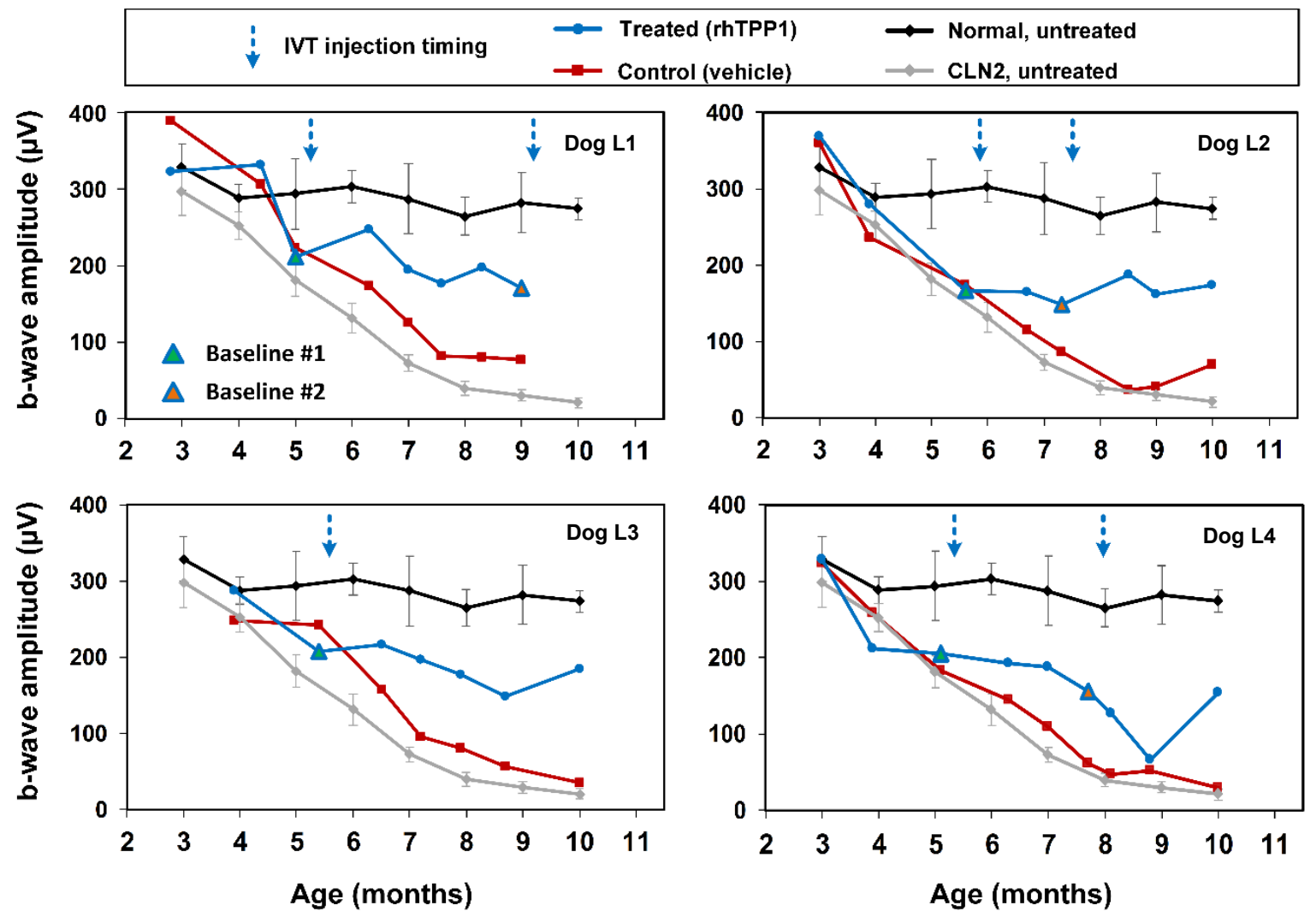

Figure 2-12: Individual mixed rod/cone responses in late started dogs L1, L2, L3 and L4. Post symptomatic intravitreal rhTPP1 halted decline of retinal mixed rod and cone responses in CLN2-affected dogs. Black and gray traces represent historical data \pm SEM from normal $(n=7)$ and CLN2-affected $(n=6)$ untreated dogs, respectively, from the same research colony. 


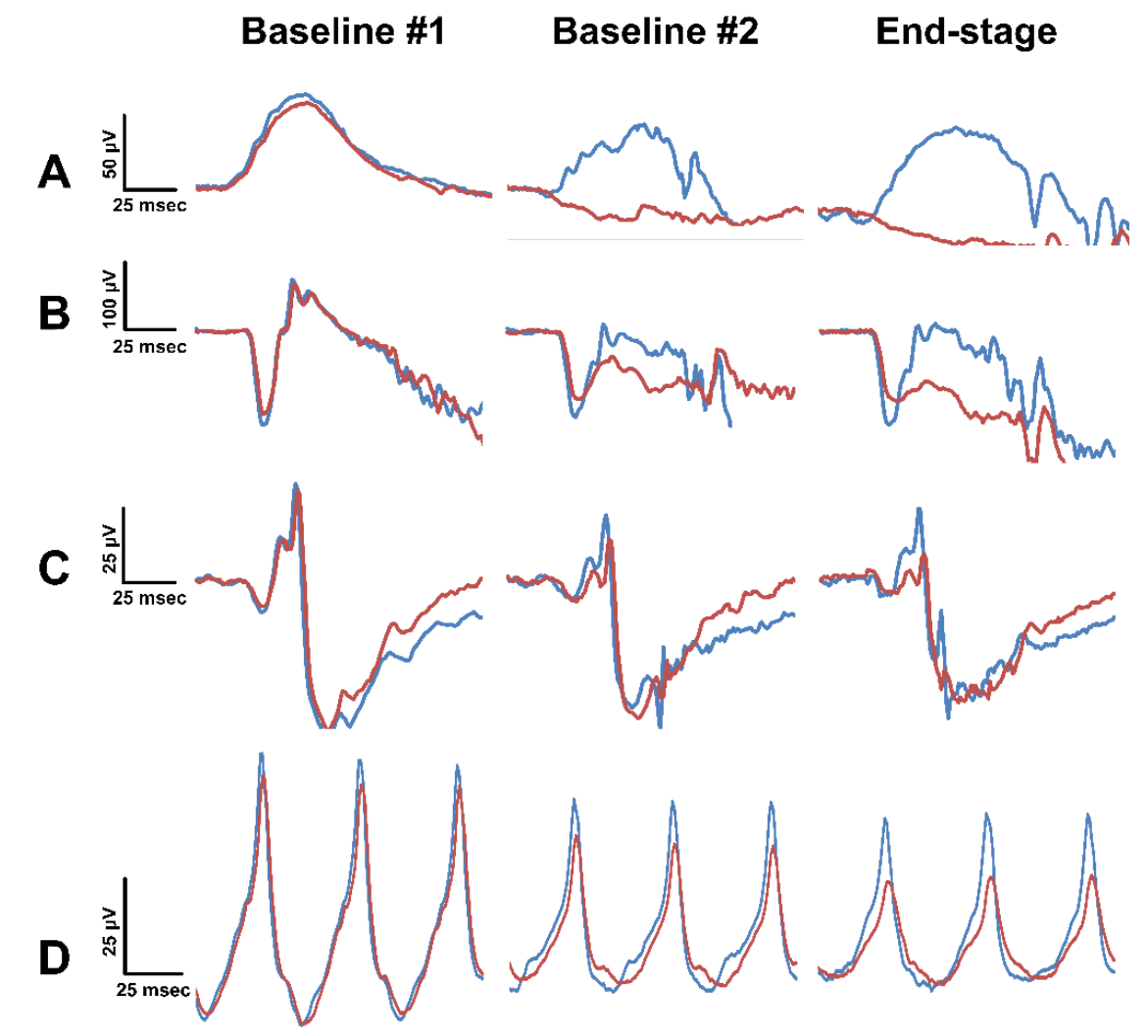

Figure 2-13: ERG waveforms from Dog $L 1$ at 5,8 , and 10 months of age. (A) pure rod, (B) mixed rod and cone, (C) pure cone, and (D) $30 \mathrm{~Hz}$ flicker responses all exhibited ERG preservation in the rhTPP1-treated eye relative to the control eye.

\subsubsection{Retinal Morphology}

As reported in previous studies, there is a thinning of both the inner and outer retina in untreated eyes of TPP 1 - Dachshunds, with the thinning being more pronounced in the inner than outer retina (Katz et al., 2008). IVT administration of rhTPP1 was effective in inhibiting this disease-related cell loss.

Quantitative analyses of the numbers of nuclei in the inner nuclear layer (INL) from several retinal regions (including central, superior mid-peripheral and inferior mid-peripheral retina) demonstrated a significant inhibition of disease- 
related loss of cells with nuclei in the INL in both early and late start dogs (Figure 2-14). At the time of euthanasia there were significantly more nuclei in all three INL regions of the rhTPP1-treated eyes than the vehicle-treated eyes of early start dogs $(p<0.05$, Student's t-test) and in the central and superior midperipheral retina (inferior mid-peripheral was not assessed) of late start dogs $(p<0.05$, Mann-Whitney Rank Sum Test).
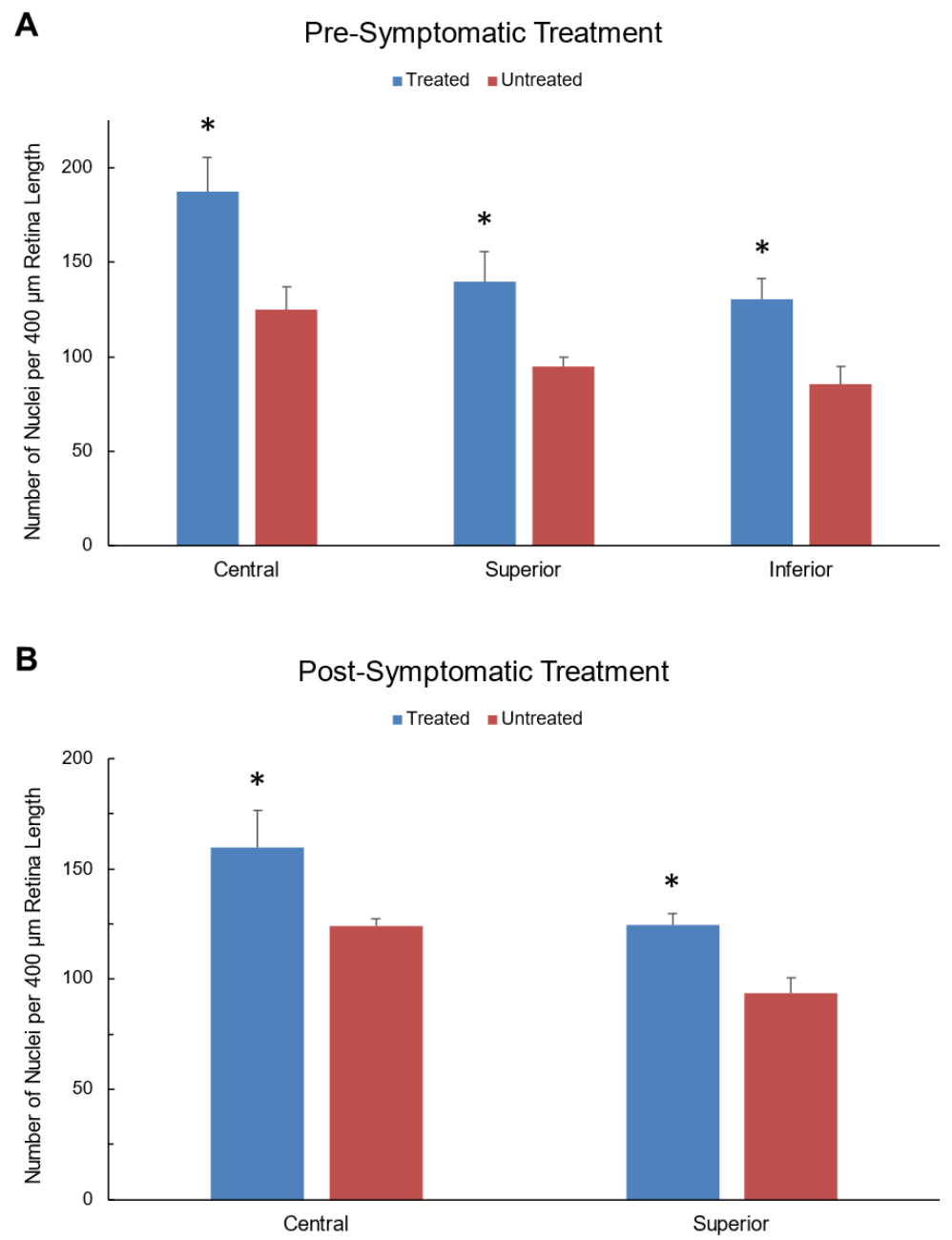

Figure 2-14: Quantification of nuclei in the inner nuclear layer from several retinal areas (central, superior mid-peripheral and inferior mid-peripheral retina) of dogs treated pre-symptomatically $(A, n=4)$ and post-symptomatically $(B, n=4)$ + SEM, ${ }^{*} \mathrm{p}<0.05$. 
Similarly, there were significantly more photoreceptor nuclei in the outer nuclear layer (ONL) of the rhTPP1-treated eyes than in the vehicle-treated eyes of late start dogs in the same two regions at the time of euthanasia (Figure 2-15, $p<0.05$, Mann-Whitney Rank Sum Test). In only one area, the superior midperipheral region of dog L1, was the cell density lower in the treated eye. However, there were no significant differences between the numbers of photoreceptor nuclei in the ONL of the early start dogs (Figure 2-15).

A Pre-Symptomatic Treatment - Treated =Untreated
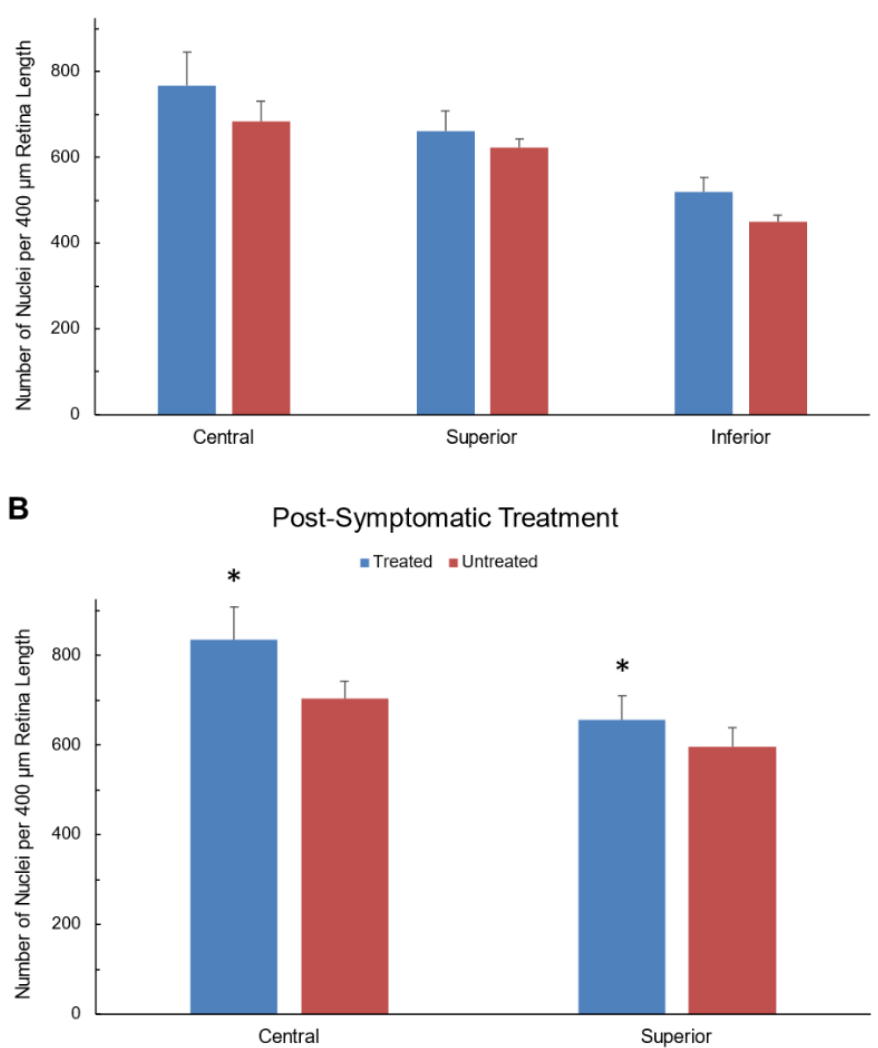

Figure 2-15: Quantification of nuclei in the outer nuclear layer from several retinal areas (central, superior mid-peripheral and inferior mid-peripheral retina) of dogs treated pre-symptomatically $(A, n=4)$ and post-symptomatically $(B, n=4)$ + SEM, ${ }^{*} \mathrm{p}<0.05$. 
Thinning of the INL and ONL are apparent in the light micrographs in Figures 2-16 and 2-17. Light micrographs also illustrate that the photoreceptor outer segments (pos) in the rhTPP1-treated eyes of early start dogs retained their normal straight and parallel morphology, whereas in the vehicle-treated eyes, the outer segments were irregular in shape and orientation (Figure 2-16, pos).
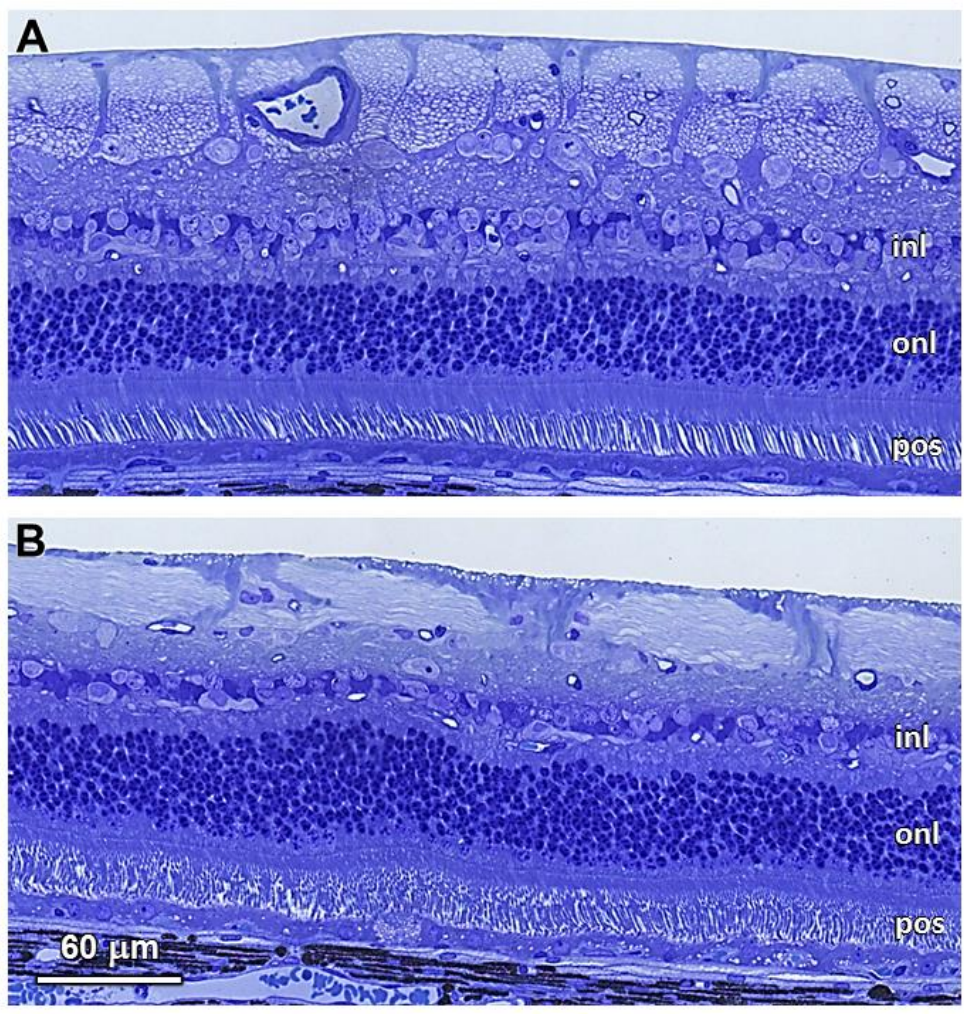

Figure 2-16: Representative light micrographs of sections from the central posterior retinas of the rhTPP1-treated (A) and vehicle-treated (B) eyes of dog E2, euthanized at end-stage disease at approximately 11 months of age. Bar in (B) indicates the magnification of both micrographs. 

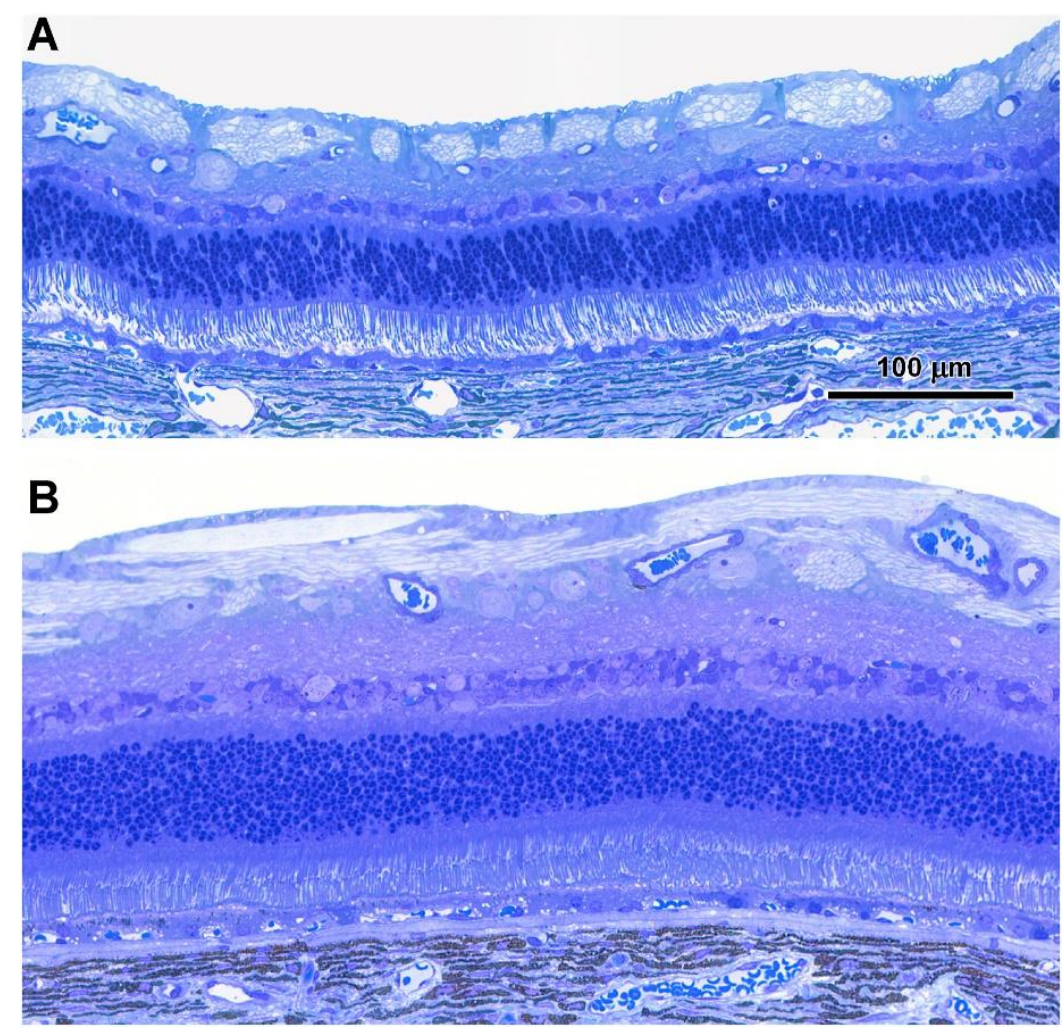

Figure 2-17: Representative light micrographs of sections from the central retinas of the vehicle-treated (A) and rhTPP1-treated (B) eyes of dog L3, euthanized at end-stage disease at approximately 10 months of age. Bar in (A) indicates the magnification of both micrographs.

Retinal ultrastructure in the central retina was assessed with electron microscopy. In the rhTPP1-treated eyes, the ultrastructure of the rod outer segments of the central retina was relatively normal. In contrast, in the vehicletreated eyes, the apical ends of the outer segments exhibited an abnormal arrangement of the disc membranes (Figures 2-18 and 2-19). 

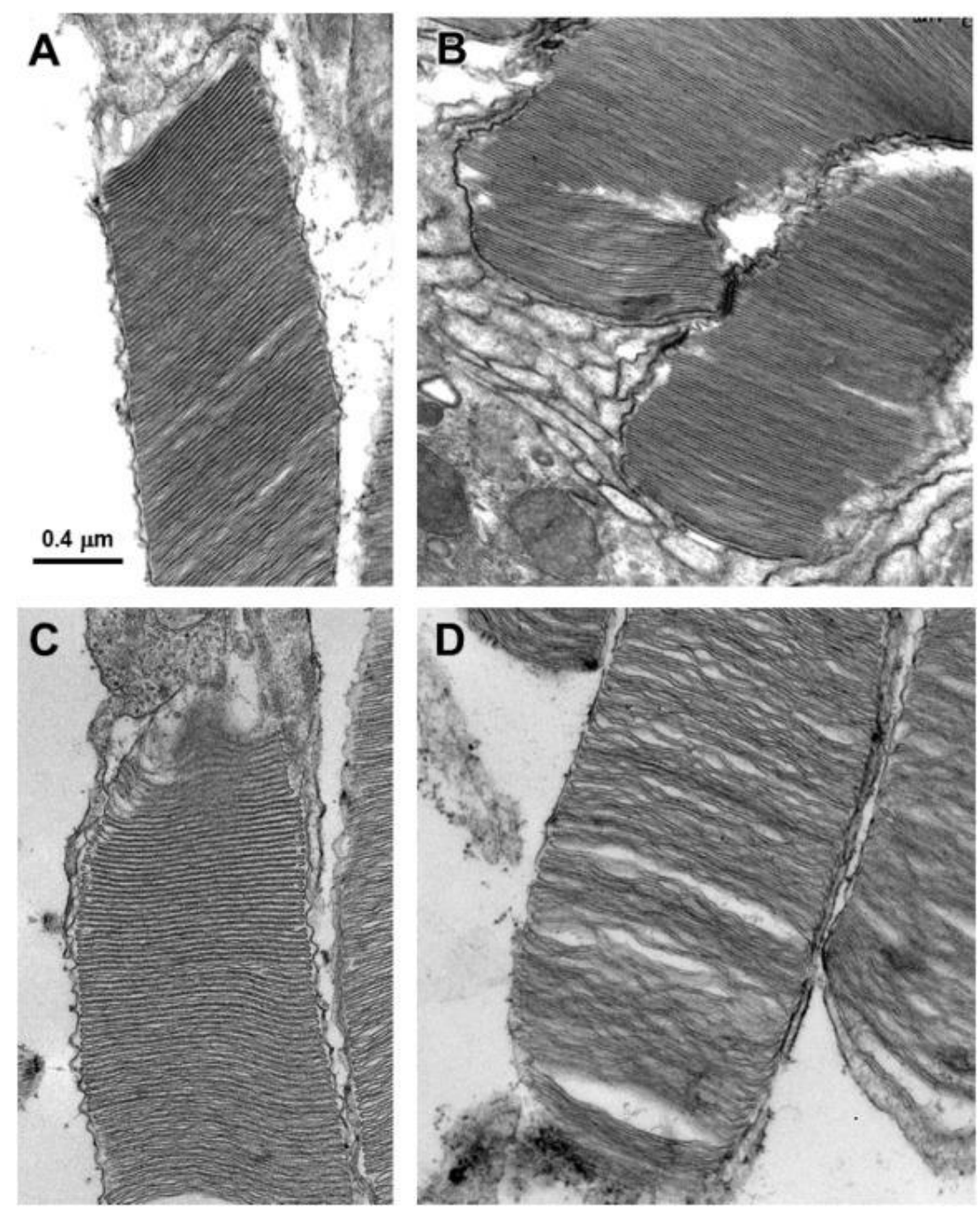

Figure 2-18: Electron micrographs of the base $(A)$ and the apical end $(B)$ of rod outer segments from the rhTPP1-treated eye and of the base $(C)$ and apical end (D) of from the vehicle-treated eye of dog E2. Normal morphology was retained at the bases of the rod outer segments in both rhTPP1- and vehicle-treated eyes (A \& C). However, while the disc membranes from the rhTPP1-treated eyes remained tightly packed, straight and parallel to one another at the distal ends of the rod outer segments, they were more loosely packed and less straight in the vehicle-treated eyes. The dog was euthanized at approximately 11 months of age. Bar in (A) indicates the magnification of all four micrographs. 


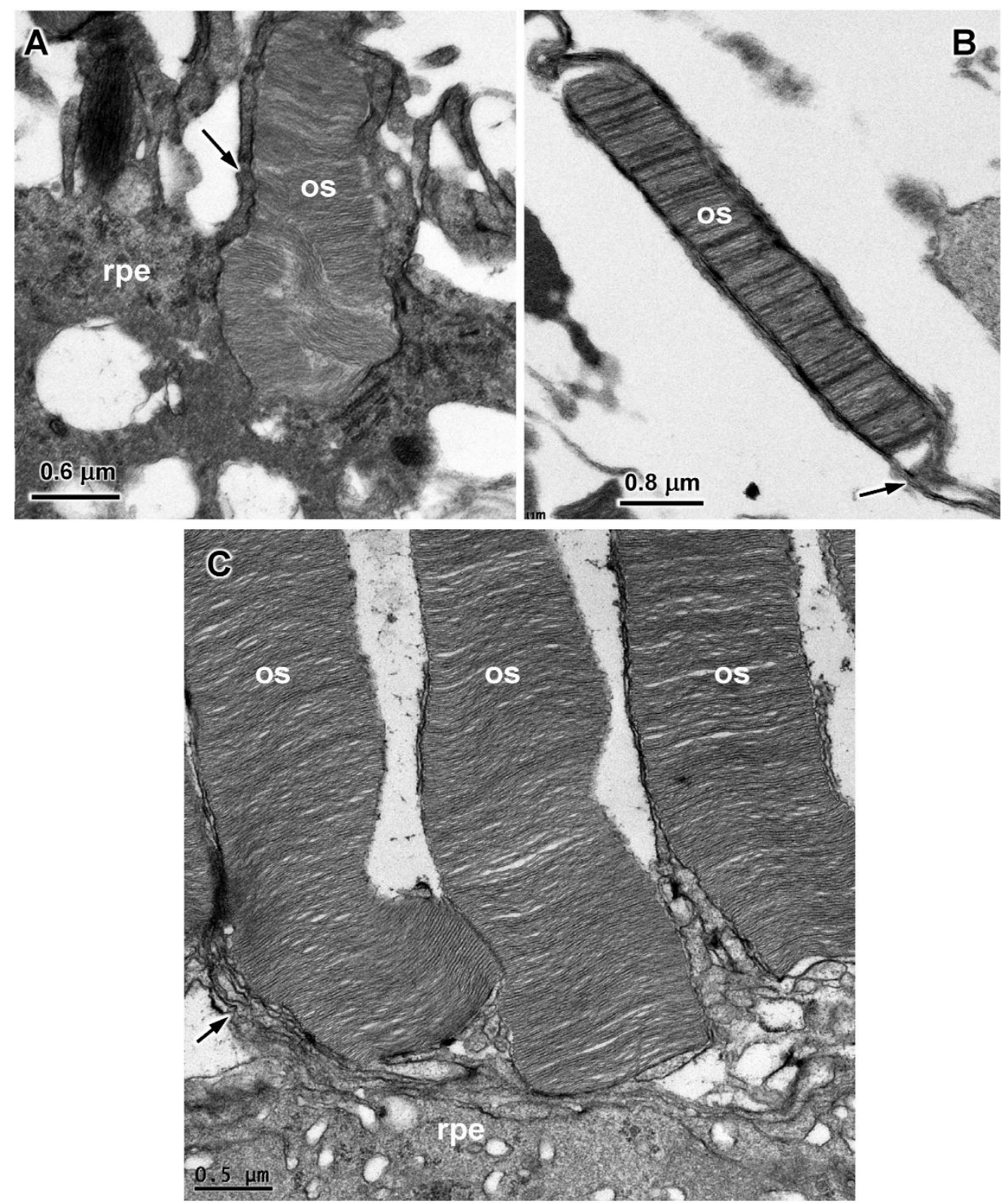

Figure 2-19: Electron micrographs of the photoreceptor-retinal pigment epithelium interfaces from the vehicle-treated (A and B) and rhTPP1-treated (C) eyes of dog L4, euthanized at approximately 10 months of age. In the rhTPP1treated eyes, the ultrastructure of the rod outer segments was relatively normal, and the distal ends of the outer segments were closely apposed to the apical side of the retinal pigment epithelium (RPE) (C). In contrast, in the vehicletreated eyes only a fraction of the outer segments extended to the RPE apical surface and the apical ends of those that did exhibited an abnormal arrangement of the disc membranes (A). Those that did not exhibited an abnormal irregularity in disc electron density and were unsheathed by long thin RPE apical processes. Rod outer segments (os); retinal pigment epithelium (rpe); rpe apical microvilli (arrows). Bar in each micrograph indicates the magnification. 
Bipolar cell axons extending from the outer plexiform layer into the ONL were quite common in rhTPP1-treated eyes but were rare in vehicle-treated eyes (Figure 2-20). In vehicle-treated eyes, axons extending in the direction of the ONL seldom penetrated into this layer, in most cases terminating at or before the inner boundary (Figure 2-21).
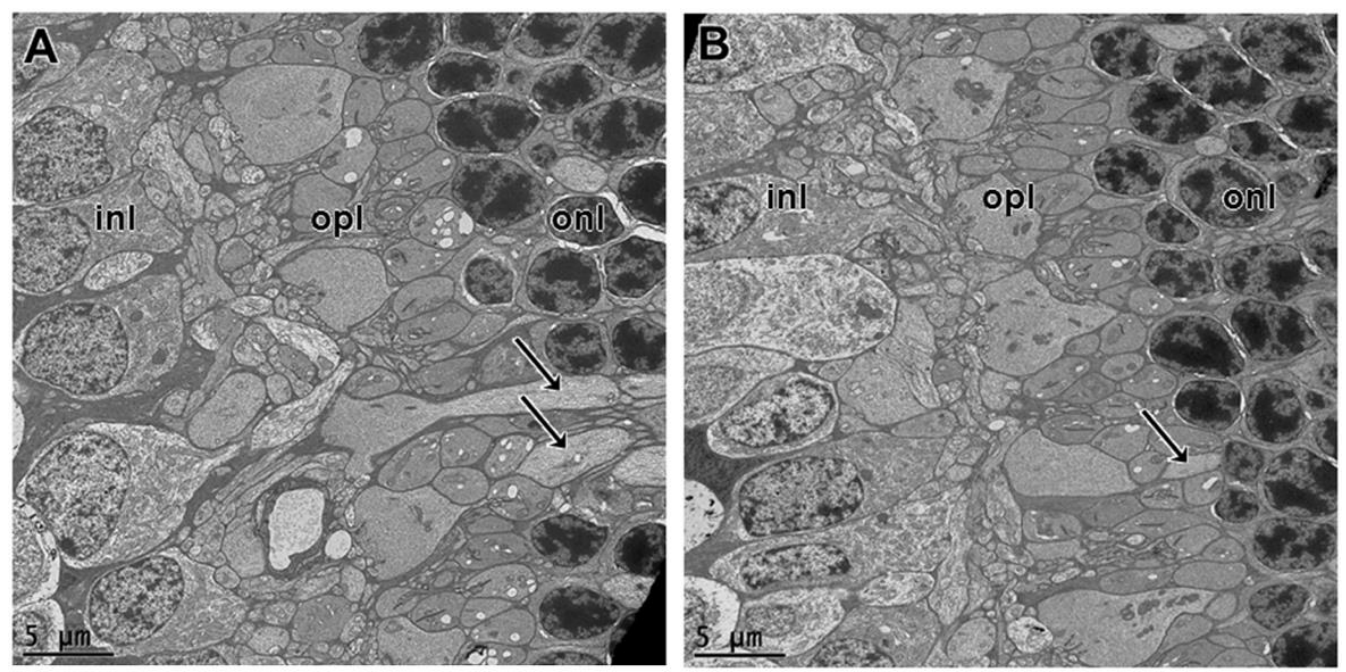

Figure 2-20: Electron micrographs of the retinal outer plexiform layer (opl) and adjacent portions of the inner nuclear layer (inl) and outer nuclear layer (onl) from the rhTPP1-treated (A) and vehicle-treated (B) eyes of dog E2 euthanized at approximately 11 months of age. Axons from inl cells are indicated by arrow. Bar in each micrograph indicates magnification. 


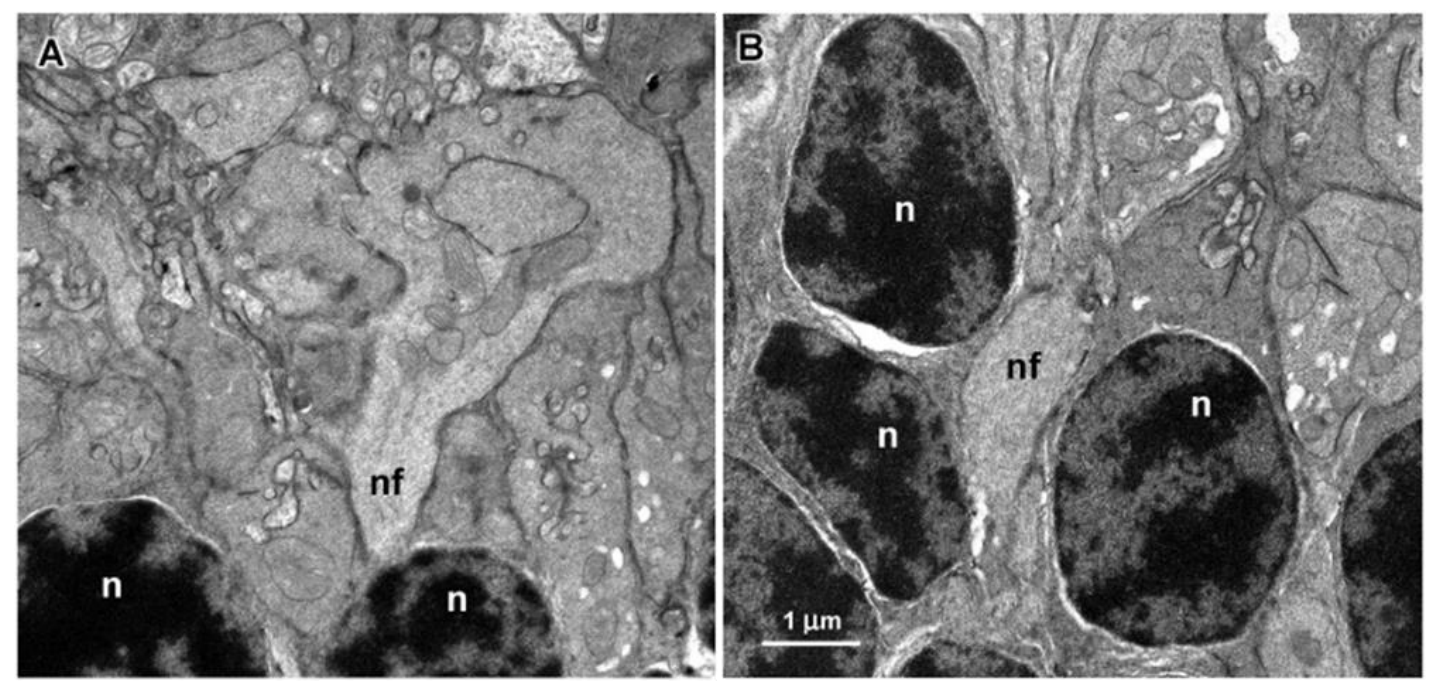

Figure 2-21: Electron micrographs of the outer plexiform layer/outer nuclear layer interface of the vehicle-treated (A) and rhTPP1-treated (B) eyes from dog L4. Rod photoreceptor nuclei (n); nerve fibers of bipolar cells (nf). Bar in (B) indicates the magnification of both micrographs.

\subsubsection{Ciliary Body Morphology}

In the rhTPP1-treated eyes, the ciliary bodies were compact and tightly adherent to the underlying sclera (Figures 2-22 and 2-23). In contrast, in the vehicle-treated eyes, the ciliary bodies were up to three times as thick as those in the rhTPP1-treated eyes, and the stroma was much more loosely organized with many acellular gaps in the tissue. In addition, except in small areas, the ciliary body was completely separated from the sclera in the vehicle-treated eyes. 

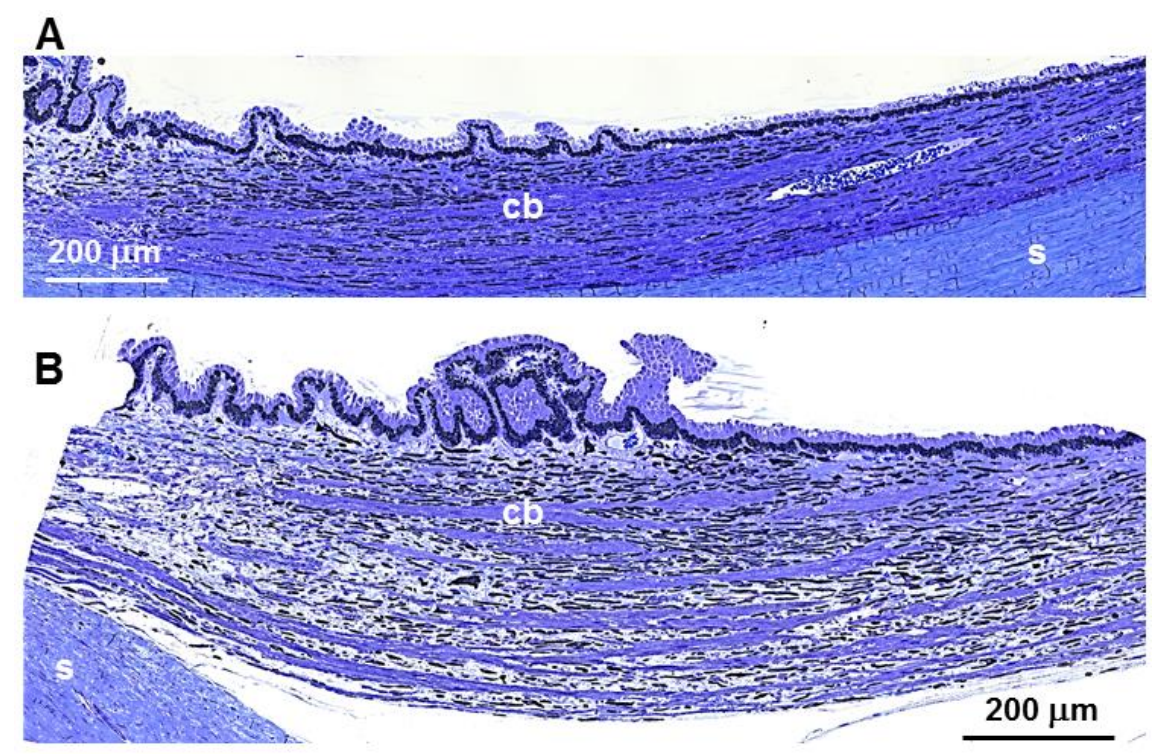

Figure 2-22: Light micrographs of the ciliary bodies (cb) and adjacent portions of the scleras (s) from the rhTPP1-treated (A) and vehicle-treated (B) eyes of dog E2 euthanized at approximately 11 months of age. Bar in each micrograph indicates the magnification.

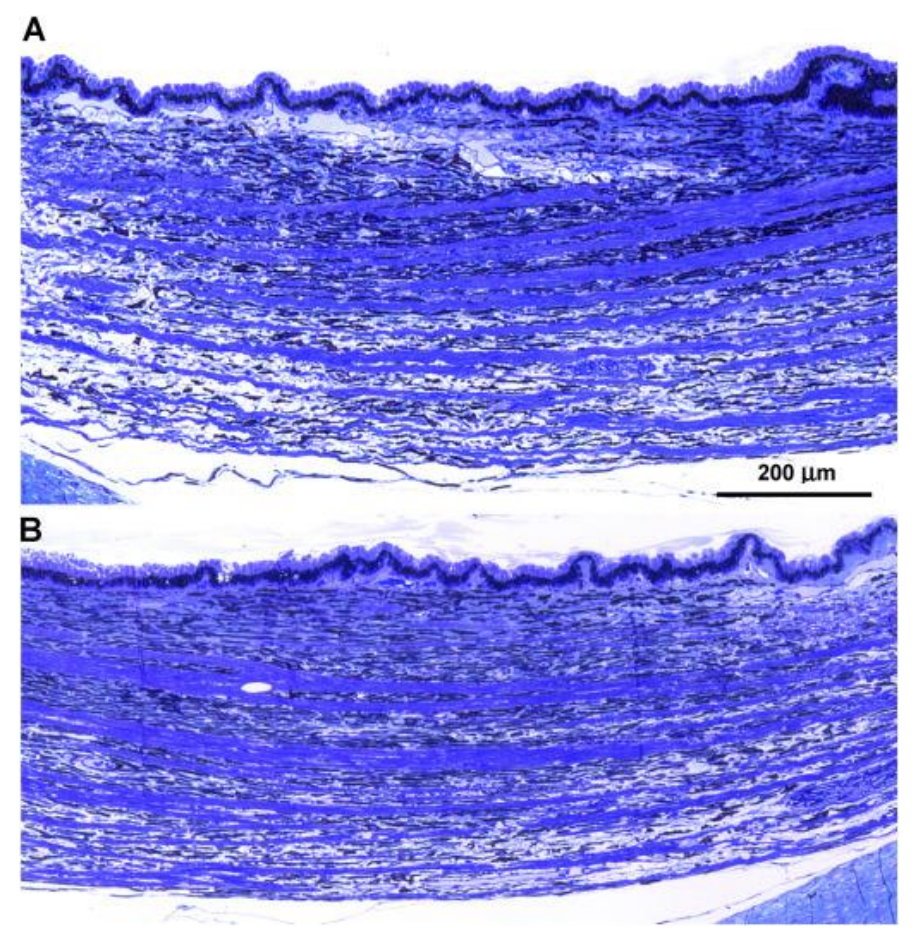

Figure 2-23: Light micrographs of the ciliary bodies from the vehicle-treated $(A)$ and rhTPP1-treated (B) eyes of dog L3 euthanized at approximately 10.5 months of age. Bar in $(A)$ indicates the magnification of both micrographs. 
Electron microscopic examination of the ciliary body of early start dogs revealed a dramatic decrease in the density of the collagen layers in this tissue in the vehicle-treated relative to the rhTPP1-treated eyes (Figure 2-24). Most of the stromal cells (s) of the sham-treated eye were very thin compared to stromal cells of the rhTPP1-treated eyes.
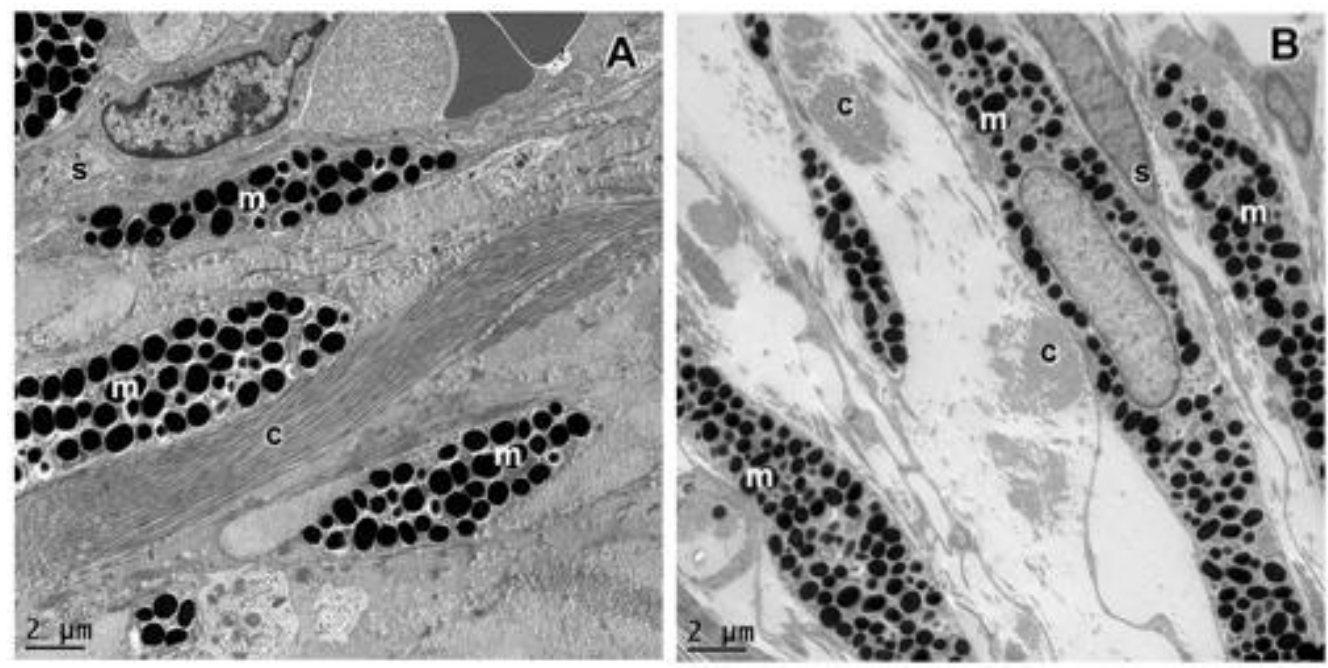

Figure 2-24: Electron micrographs of areas of the ciliary bodies from the rhTPP1-treated (A) and vehicle-treated (B) eyes of dog E2 illustrating the tight packing between the cells and extracellular matrix in the treated eye and the presence of large gaps within the tissue of the vehicle-treated eye associated with a decrease in collagen (c) density, and a thinning of the stromal cell bodies (s). melanocytes $(\mathrm{m})$. Bar in both micrographs indicates the magnification.

\subsubsection{Indirect Assessment of Ganglion Cell Numbers}

Since each retinal ganglion cell has one axon that traverses the optic nerve, the numbers of ganglion cells in the eyes of late start dogs were determined indirectly by counting the numbers of axons in stained cross-sections of the optic nerve from each eye. A representative image of an optic nerve section used for these analyses is shown in Figure 2-25. The difference in axon numbers between 
the rhTPP1-treated and vehicle-treated eyes (Table 2-6) was not statistically significant ( $p=0.07$, t-test), but the power of the statistical comparison with $\alpha=$ 0.05 was only 0.36 , so the data are not sufficient to rule out that rhTPP 1 treatment was effective in inhibiting disease-related loss of ganglion cells, or at least of their axons.

\begin{tabular}{|c|c|c|c|}
\cline { 2 - 3 } \multicolumn{1}{c|}{} & Number of Optic Nerve Axons & \multicolumn{1}{c}{} \\
\hline Dog & $\begin{array}{c}\text { rhTPP1 } \\
\text { Treated Eye (T) }\end{array}$ & $\begin{array}{c}\text { Vehicle } \\
\text { Treated Eye (C) }\end{array}$ & C/T x 100 \\
\hline L1 & 141,438 & 125,177 & 88.5 \\
\hline L2 & 141,074 & 141,161 & 100.1 \\
\hline L3 & 139,596 & 133,182 & 95.4 \\
\hline L4 & 153,289 & 136,057 & 88.8 \\
\hline
\end{tabular}

Table 2-6: Numbers of axons in the optic nerves of late start dogs 

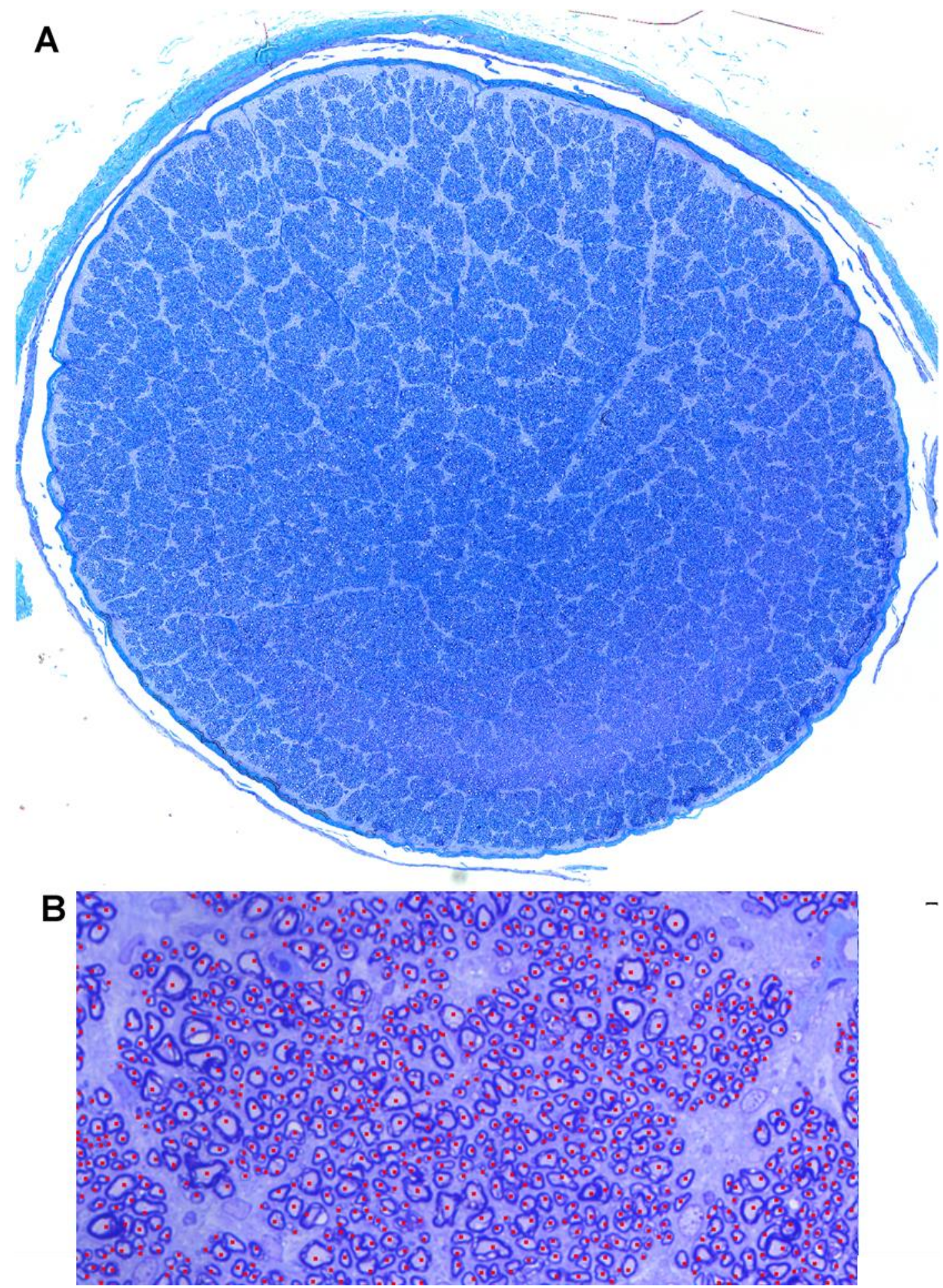

Figure 2-25: Merged light microscopic image of an optic nerve cross-section from the rhTPP1-treated eye of dog L1 (A) and an enlarged area of this image showing counting of individual axons (B). 


\subsubsection{Retinal Ganglion Cell Storage Body Content}

Canine CLN2 disease is characterized by an abundant accumulation of lysosomal storage bodies in the retinal ganglion cells. To determine whether IVT rhTPP1 treatment was effective in ameliorating this accumulation, the fraction the cytoplasmic area composed of storage bodies was assessed in thin sections of the central retina from each eye of each dog. At end-stage disease the ganglion cell storage body content of the rhTPP1-treated eyes was on average only $12 \%$ of that of the vehicle-treated eyes (Table 2-7). The storage bodies were dramatically smaller and fewer in number in the rhTPP1-treated eyes than in the vehicle-treated eyes (Figure 2-26).

\begin{tabular}{|c|c|c|c|}
\cline { 2 - 3 } \multicolumn{1}{c|}{} & \multicolumn{2}{c}{$\begin{array}{c}\text { Storage Body Content, Mean + SE } \\
\text { (\% cytoplasmic area occupied by storage } \\
\text { bodies) }\end{array}$} & \multicolumn{1}{c}{} \\
\hline Dog & rhTPP1-Treated Eye & Vehicle-Treated Eye & P value \\
\hline L1 & $2.1 \pm 0.3$ & $26.8 \pm 3.5$ & $<0.001$ \\
\hline L2 & $6.2 \pm 1.8$ & $26.0 \pm 5.8$ & 0.002 \\
\hline L3 & $2.7 \pm 0.7$ & $29.5 \pm 2.3$ & $<0.001$ \\
\hline L4 & $2.7 \pm 0.7$ & $34.9 \pm 7.9$ & $<0.001$ \\
\hline
\end{tabular}

Table 2-7: Effect of rhTPP1 treatment on storage body content of retinal ganglion cells 

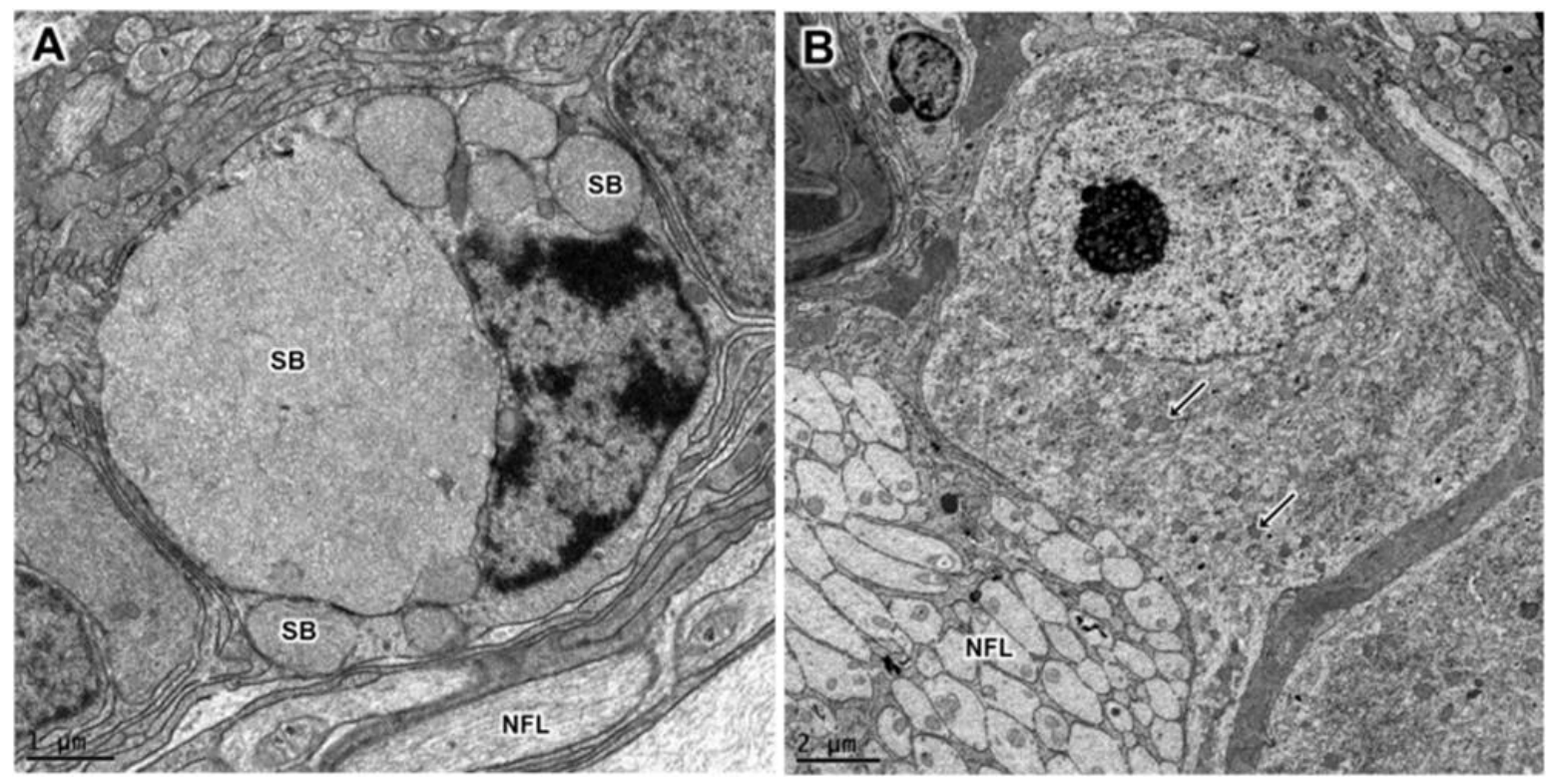

Figure 2-26: Electron micrographs of retinal ganglion cells from the vehicletreated eye (A) and the rhTPP1-treated eye (B) from dog L4. Ganglion storage bodies "SB" and arrows, nerve fiber layer "NFL". Bar in each micrograph indicates the magnification.

\subsection{Discussion}

This study demonstrates that repeated intravitreal injections of rhTPP1 are effective in inhibiting retinal degeneration and preserving retinal function in canine CLN2 disease when the treatment is initiated before any signs of retinal degeneration or loss of function are detectable (Whiting et al., 2020a). Additionally, treatment is able to halt disease-related retinal pathology when initiated after partial declines in visual function have already occurred (Whiting et al., 2020b). Pre-symptomatic initiation of treatment resulted in full preservation of b-wave amplitudes in dogs that received the full treatment course while initiation of treatment after onset of retinal decline was able to prevent further decline. Both pre- and post-symptomatic treatment preserved retinal morphology through end-stage disease. Treatment also preserved ciliary body morphology. The 
cause of the changes seen in the ciliary bodies of untreated eyes is unknown. While this could be due to lack of TPP1, anterior segment changes including alterations to the ciliary body thought to be secondary to retinal degeneration have been observed in models of inherited retinal dystrophies and in the eyes of patients with retinitis pigmentosa (Horneber et al., 1996; May et al., 2009, 2005; Schreckenberger et al., 1994; Yamaguchi et al., 1991). Given that pathology at this disease stage seems to be otherwise limited to neurons, it may be more likely that these ciliary body changes are secondary, suggesting that treatment prevented not only retinal degeneration but also its downstream consequences.

In dogs E2 and E3, injections performed once every 3 weeks provided adequate TPP1 delivery to maintain normal or above normal ERG amplitudes; however, dosing every 3 weeks resulted in recurrent inflammatory reactions. To determine whether less frequent dosing could achieve a similar therapeutic benefit with less intraocular inflammation, dog E4 was treated at 6 week intervals, and this less frequent dosing was just as effective at preserving retinal function while substantially reducing the severity and duration of post-injection intraocular inflammation. In addition to inflammatory reactions, frequent intravitreal injections are associated with risks of other complications and it is beneficial to maximize the interval between doses and reducing the total number of injections. Data from dog E1 suggested that optimization of the dosing regimen should be possible by determining the minimum dosing frequency and amount of rhTPP1 per dose necessary to maintain normal ERG amplitudes while minimizing or eliminating inflammatory responses. While short-term dosing early 
in the disease course in this dog was not sufficient to completely preserve normal retinal function, the eye that received a few early doses of rhTPP1 exhibited ERG amplitudes that were greater than those of the vehicle-treated eye for 6 months after the rhTPP1 injections were discontinued. In fact, in the late start study, a single intravitreal injection of rhTPP1 in a 25 week old dog (L3) was effective in preserving retinal function and structure to end-stage disease at 45 weeks of age. In this dog, there was essentially no decline in ERG b-wave amplitudes between the time of the single injection and euthanasia, indicating that the treatment effect lasted at least this long. Because the dogs succumbed to the neurological disease at $40-45$ weeks of age, we were unable to determine the maximum duration of the treatment effect.

The only complication of the treatments was transient intraocular inflammation after all but the first rhTPP1 injection which was not prevented by immunosuppressant treatments. While the eye is widely considered to be an immune-privileged site, other studies have demonstrated that there are limits to this privilege (Caspi, 2010; Caspi et al., 2008; Lee et al., 2014; Marticorena et al., 2012; Trivizki et al., 2018). Inflammation was likely mediated by a systemic immune reaction to the rhTPP1, not unexpected since rhTPP1 is a foreign protein for dogs with a null mutation in the TPP1 gene. Anti-TPP1 antibody titers support the idea that the dogs mounted a systemic immune response to the injected protein. The late start dogs that received two rhTPP1 injections had very high plasma anti-TPP1 antibody titers; however, titers were low in the dog that received a single rhTPP1 injection, suggesting that the first injection primed the 
immune system to respond to the subsequent exposure to rhTPP1. In addition, the inflammatory response was observed only in the eyes treated with rhTPP1 and not in the contralateral eyes injected with vehicle. The inflammation was likely due, at least in part, to rhTPP1 within the retina, since in the affected eyes there was perivascular cuffing around the retinal vessels indicating a migration of inflammatory cells into the retina. Although the intraocular inflammation was an adverse reaction, it only transiently interfered with therapeutic benefit on the ERG. Other studies have similarly observed recovery of the ERG and visual acuity after resolution of an inflammatory episode, which is encouraging and supports the idea that transient inflammation does not appear to cause long-term damage to the retina. The dog eye may be more susceptible to developing immune-mediated uveitis than eyes of other species. The experience of contract research companies that have performed numerous preclinical studies in dogs for pharmaceuticals that have subsequently undergone human clinical trials indicates that treatment-related inflammatory responses that occur in dogs are seldom recapitulated in human subjects (personal communications). Inflammatory changes observed in preclinical studies using heterologous protein are well known and these inflammatory findings have not translated to humans with a variety of approved drugs administered via intravitreal injection (Krzystolik et al., 2002; Wakshull et al., 2017). However, inflammation could be a concern, especially for patients with two null mutations in TPP1. In the canine CNS ERT study, the lab showed that tolerization to TPP1 was possible by gradually increasing the amount of TPP1 infused. The dog treated with the highest dose 
experienced an anaphylactic reaction during the second infusion but following a tolerizing dosing regimen was able to safely receive the full dose for the rest of the study (Katz et al., 2014). Therefore, it may also be possible to induce tolerance in children, particularly since children receiving intravitreal ERT will also be receiving the CNS ERT.

CLN2 is an example of a disease that will require targeting therapies to multiple organ systems to effectively treat the entire disorder. The treatment described in this study may be an effective adjunct to ICV infusion of rhTPP1 that has been shown to be effective in preserving CNS structure and function in the Dachshund CLN2 disease model. The CNS treatment did not preserve retinal structure or function in this model, presumably because little if any of the rhTPP1 infused into the CSF reaches the retina beyond possibly the retinal ganglion cells. Direct targeting of the retina with IVT administration in combination with ICV administration will probably be necessary in order to preserve vision. However, a combined treatment regimen could be accompanied by more severe intraocular inflammation than was observed in this study. Further investigation will be necessary to determine the safety and efficacy of combined CNS and retinal therapy. The data presented here support further investigation to evaluate intravitreal enzyme replacement as a potential treatment to preserve vision in CLN2 disease. These results have been incorporated into an application to the Food and Drug Administration and European regulatory agencies for approval of this as an adjunct treatment to prevent progressive vision loss in children with CLN2 disease that are undergoing CNS enzyme replacement. 


\section{Implantation of TPP1-Overexpressing MSCs Preserves}

\section{Retinal Function but did not Prevent Neurological Disease}

\section{Progression}

\subsection{Introduction}

TPP1 enzyme replacement therapy is an effective treatment for preserving central nervous system and retinal function; however, one drawback to these therapies is the lifelong need for periodic intravitreal injections and intracerebroventricular infusions. While intravitreal injections are a common outpatient procedure and ICV ERT has been shown to be safe when best practice guidelines are followed, both do entail procedure-related risks in addition to great expense and time cost (Boyle et al., 2018; Falavarjani and Nguyen, 2013; Lewis et al., 2019; Schwering et al., 2020). Therefore, we sought to develop a way to deliver a long-term source of TPP1 to the brain and retina that would eliminate the need for repeated injections. In this study, we tested cellmediated ex vivo gene therapy.

A concern with biotherapeutics like rhTPP1 or gene therapy products is the potential immunogenicity of the protein or viral capsid. Immune responses against the recombinant protein including anti-TPP1 antibody formation, mild infusion associated reactions and anaphylactic reactions were observed when rhTPP1 was administered to TPP1-null Dachshunds via infusion into the CSF (Katz et al., 2014; Vuillemenot et al., 2015, 2011). Dogs treated with CSF 
infusions of AAV2.TPP1 produced anti-TPP1 antibodies and intravitreal administration of rhTPP1 resulted in anti-TPP1 antibody production and intraocular inflammation (Katz et al., 2015; Whiting et al., 2020b, 2020a). Although some children who received CSF infusions of rhTPP1 developed antiTPP1 antibodies, this was fortunately not associated with severe hypersensitivity reactions or with decreased treatment efficacy (Cherukuri et al., 2018). However, intraocular inflammation has been a concern in clinical trials of gene therapies for inherited retinopathies (Bainbridge et al., 2015; Cukras et al., 2018; Dimopoulos et al., 2018).

Mesenchymal stem cells are of clinical interest due to their immunosuppressive properties. It seemed possible that a slow, continuous release of human TPP1 (hTPP1) into the vitreous from implanted hTPP1producing MSCs (TPP1-MSCs) paired with the innate immunosuppressive properties of MSCs could provide a long-term source of TPP1 to the retina while mitigating or preventing treatment-related inflammation. Indeed, in previous trials treatment with TPP1-MSCs improved retinal function and structure without causing intraocular inflammation (Tracy et al., 2016). Therefore, we investigated whether TPP1-MSCs could have a similar therapeutic benefit in the CNS. We hypothesized that hTPP1-overexpressing autologous MSCs could provide sustained, continuous delivery of TPP1 to both the brain and retina, delay neurological disease progression and preserve retinal function without the need for repeated administration or the inflammatory response associated with ERT or in vivo gene therapy. 


\subsection{Methods}

\subsubsection{Animals}

The dogs used for this study were TPP1- purpose-bred miniature longhaired Dachshunds $(n=9)$ housed in a research colony at the University of Missouri - Columbia. Breeding, genotyping, and husbandry were performed as described in 2.2.1. The study was approved by the University of Missouri Animal Care and Use Committee.

\subsubsection{Neurologic and Ophthalmic Examinations}

Complete ophthalmic exams were performed prior to enrollment in the study at approximately 10-12 weeks of age. Dogs with any ophthalmic abnormalities that could compromise vision were excluded from the study. Ophthalmic exams were performed as described in 2.2.2.

Neurologic and physical examinations were performed weekly, beginning at 10 weeks of age. Body weights were recorded weekly. Neurological exams were videotaped every other week starting at 8 months of age or at the onset of neurologic signs, whichever occurred first. A standard clinical neurological exam was used (Dewey et al., 2015; Lorenz et al., 2011). The exam included observation of mentation, movement abnormalities (intention tremor, resting tremor, myoclonus), seizure activity, posture (wide-based stance, falling) and gait (ambulatory or non-ambulatory paresis; general proprioceptive, vestibular or cerebellar ataxia). Postural reactions (proprioception/knuckling and hopping) were assessed in all four limbs as normal, delayed or absent. Cranial nerves 
were evaluated, and the reflex or response was assessed as normal or absent. Spinal reflexes (withdrawal reflex, patellar reflex, perineal reflex, cutaneous trunci reflex) and sensation were evaluated.

\subsubsection{Isolation, Culture and Transduction of MSCs}

Bone marrow was collected from the humerus at 2.5 to 4 months of age. Dogs were sedated with intramuscular injections of $5 \mu \mathrm{g} / \mathrm{kg}$ dexmedetomidine hydrochloride and $0.025 \mathrm{mg} / \mathrm{kg}$ buprenorphine. IV propofol was given to effect and dogs were then intubated and maintained under general anesthesia with isoflurane. $0.8 \mathrm{~mL}$ lidocaine with $0.2 \mathrm{~mL}$ sodium bicarbonate was administered to the biopsy area by intramuscular injection immediately before the biopsy.

The skin over the proximal end of the humerus was clipped, shaved and aseptically prepared and the bone marrow aspiration was performed using sterile procedures. An incision was made over the proximal portion of the greater tubercle of the humerus using a \#15 surgical blade. An 11-gauge $10 \mathrm{~cm}$ Jamshidi needle was then introduced through the incision and passed into the bone at the fossa where the lateral glenohumeral ligament attaches at such an angle that the needle fully penetrated the cortex and was advanced into the medullary canal. The stylet was removed and a $10 \mathrm{~mL}$ syringe containing $0.2 \mathrm{~mL}$ heparin was attached to the needle. $5 \mathrm{~mL}$ of marrow was withdrawn while continuously rotating the needle to prevent clot formation. In a sterile hood, the bone marrow aspirate was split between two sterile $15 \mathrm{~mL}$ conical tubes each containing 3-5 
$\mathrm{mL}$ of sterile Minimal Essential Medium alpha modification (MEM $\alpha$; GE Healthcare HyClone, Chicago, IL).

All cell culture procedures were done under sterile conditions in a cell culture hood using sterile supplies. The tubes containing the bone marrow aspirate and MEM $\alpha$ were spun for 10 minutes at $1591 \mathrm{xg}$ (Unico Powerspin centrifuge, "Blood" preset). In a cell culture hood, the supernatant was removed with a sterile serological pipette attached to a vacuum aspirator and the pellet was resuspended in approximately $5 \mathrm{~mL}$ of bone marrow culture medium containing either $10 \%$ (BMC-10) or $20 \%$ (BMC-20) fetal bovine serum (FBS) and $1 \%$ penicillin/streptomycin in MEM $\alpha$. For all culture procedures, characterized US origin FBS (GE Healthcare HyClone, Chicago, IL) was used for dogs 1-5 and defined US origin gamma irradiated FBS (GE Healthcare HyClone, Chicago, IL) was used for dogs 6-9. The suspension was then pipetted onto a $100 \mathrm{~mm} \times 20$ $\mathrm{mm}$ cell culture dish and $10 \mathrm{~mL}$ of $\mathrm{BMC}$ medium was added. The passage 0 (P0) dishes were placed in an incubator at $37^{\circ} \mathrm{C}$ with $5 \% \mathrm{CO}_{2}$ injection. After 24 hours, the cells were washed and spent media was replaced. Spent media was aspirated and the plates were washed three times with $5 \mathrm{~mL}$ of sterile $1 \mathrm{X}$ phosphate buffered saline (PBS) to remove as many non-adherent cells as possible then fed by adding $10-12 \mathrm{~mL}$ of BMC medium. The plates were returned to the incubator. At this point, a few individual MSCs could typically be noted on the plates.

After the initial plating, PO MSCs were maintained in culture and expanded to obtain sufficient numbers for implantation. The P0 cells were washed and 
spent media replaced every 2-4 days. After several washes, non-adherent cells were lost and only adherent, fibroblast-like MSCs remained. Colonies of MSCs derived from the initially plated cells could be observed. Once the cells reached confluency in the $100 \mathrm{~mm} \times 20 \mathrm{~mm}$ dish, they were passaged to a larger $150 \mathrm{~mm}$ x $20 \mathrm{~mm}$ culture dish.

For passaging, the plates were washed with $5 \mathrm{~mL} 1 \mathrm{X}$ PBS followed by addition of a dissociation reagent to remove adherent MSCs from the plate. Several dissociation reagents were used over the course of these studies: Gibco 0.05\% trypsin-EDTA phenol red (Thermo Fisher Scientific, Waltham, MA), HyQTase (GE Healthcare HyClone, Chicago, IL), Accutase (Innovative Cell Technologies, San Diego, CA) and Gibco TrypLE Select without phenol red (Thermo Fisher Scientific, Waltham, MA). 0.05\% trypsin and HyQTase were stored frozen and thawed and warmed in a $37^{\circ} \mathrm{C}$ water bath prior to use. Accutase was stored at $4^{\circ} \mathrm{C}$ and used at this temperature. TrypLE Select was stored protected from light and stored and used at room temperature. $3 \mathrm{~mL}$ of dissociation reagent was added to each $100 \mathrm{~mm}$ plate. The plates were briefly placed in the incubator, then removed and agitated by gently hitting against the countertop and swirling. The cells were visually evaluated for detachment using a microscope to ensure that the majority of the cells were free-floating and no longer adhered to the dish. Incubation times varied for each dissociation reagent. $0.05 \%$ trypsin was applied for 5 minutes initially and was left on for up to 15 minutes. HyTQase was left on for up to 45 minutes and Accutase up to 20 minutes. TrypLE Select was left on until cells were detached, typically 15-20 
minutes. Once the cells were in solution, the dissociation reagent was inactivated by addition of $12 \mathrm{~mL}$ BMC medium (four times the amount of dissociation reagent applied). The solution was gently but thoroughly mixed with a $25 \mathrm{~mL}$ sterile serological pipette and transferred to a fresh $150 \mathrm{~mm}$ plate. Additional BMC medium was added to equal a total of $25 \mathrm{~mL}$ per $150 \mathrm{~mm}$ plate and the $\mathrm{P} 1$ cells were returned to the incubator.

After reaching confluency in the $150 \mathrm{~mm}$ plates, the cells were passaged again as described, except that $8 \mathrm{~mL}$ of dissociation reagent was used and deactivated with $32 \mathrm{~mL} \mathrm{BMC}$ medium and the solution was mixed with a $50 \mathrm{~mL}$ pipette and split equally between two new $150 \mathrm{~mm}$ plates (1:2). At this point, cells were maintained with washing and media replacement every $2-4$ days and passaging at 1:2 when the plates reached confluency. Depending on the growth rate of the cells, plates were occasionally passaged at a lower density. The cells were expanded until the required number of plates was obtained.

For eye treatments, all dogs received GFP-MSCs (transduced with AAV2.CAG.GFP) in the right eye and TPP1-MSCs (transduced with AAV2.CAG.hTPP1) in the left eye. For CNS treatments, TPP1-MSCs (AAV2.CAG.hTPP1) and mCherry-MSCs (AAV2.CAG.mCherry) were used. When a portion of the TPP1-MSCs were co-transduced to express hTPP1 and a fluorophore, AAV5.CAG.GFP and AAV5.CAG. mCherry were used. All vectors were obtained from SignaGen Laboratories (Rockville, MD).

MSCs were transduced seven days before the scheduled injection to allow time for vector expression. The viral stock was briefly thawed at room 
temperature and centrifuged. The required amount of viral stock solution (Appendix A) was added to a tube containing a volume of medium equal to $2 \mathrm{X}$ the number of plates to be transduced and mixed thoroughly. $2 \mathrm{~mL}$ of the viruscontaining medium was added to each plate. The cells were then returned to the incubator for 72 to 96 hours. After this time, the plates were washed and media replaced to remove the vector. In the case of cells that were co-transduced with a fluorescent marker, the vector (AAV5.CAG.mCherry or AAV5.CAG.GFP) was applied as described during media replacement following removal of the AAV2 vector solution and the AAV5 vector solution was removed after 24-48 hours. Otherwise, cells were maintained as usual until the day of injection. Plates from which media samples were to be taken for TPP1 concentration determination were washed and fed 24 hours before sample collection. Media samples were stored at $-80^{\circ} \mathrm{C}$.

MSCs were collected immediately prior to injection. GFP expression in GFP-MSCs was verified with a fluorescence stereoscope and media samples from both TPP1-MSCs and GFP-MSCs were collected before beginning. Cells were washed and the dissociation reagent applied as described previously. After the dissociation reagent was inactivated, the entire cell suspension was transferred to a $50 \mathrm{~mL}$ conical tube and spun at 2,000 RPM in a Sorvall RC5B centrifuge with a fixed-angle rotor for 8 minutes. The supernatant was carefully aspirated from each tube, leaving the cell pellet intact. The pellets were then sequentially resuspended in MEM $\alpha$ by adding a small amount of MEM $\alpha$ to the first tube and gently tapping the side of the tube to resuspend the pellet and 
transferring the suspension to the next tube to resuspend subsequent pellets, being careful not to over-mix or lyse the cells. The entire cell suspension was collected in a sterile tube for injection and $15 \mu \mathrm{L}$ of the suspension was removed for determination of the total number of cells collected.

MSCs were counted by diluting $15 \mu \mathrm{L}$ of the suspension $1: 40$ in dissociation reagent and incubating on a shaker for at least 15 minutes to break any clumped cells into a countable single cell suspension. Triplicate cell counts were then obtained with a handheld Scepter Coulter Counter (MilliporeSigma, Burlington, MA). If the cell count was too high for the Scepter Counter, the suspension was further diluted to 1:80 with $1 \mathrm{X}$ PBS.

\subsubsection{Treatments}

Dogs received TPP1-MSCs to treat the eye, brain or a combined therapy. Treatments are summarized in Table 3-1. Dogs 1-4 and 9 received combined therapy: TPP1-MSCs were administered to the left eyes via intravitreal (IVT) injection; the right eyes received IVT injections of GFP-MSCs; and TPP1-MSCs, sometimes including cells co-transduced to express a fluorescence marker, were administered to the CSF via injection at the cisterna magna (CM) or the lateral ventricles (ICV). Dog 5 received CM injections of TPP1-MSCs and did not receive eye treatments. Dog 6 received eye treatments as well as a CM injection of mCherry-MSCs to assess survival and distribution of the cells in vivo. Dog 7 received only eye treatments. Additional cell culture details can be found in Appendix A. 


\begin{tabular}{|c|c|c|c|c|c|}
\hline Dog ID & $\begin{array}{c}\text { Age } \\
\text { (months) }\end{array}$ & $\begin{array}{c}\text { Dose (\# of } \\
\text { TPP1-MSCs) }\end{array}$ & $\begin{array}{c}\text { Route of } \\
\text { Administration }\end{array}$ & Transgene & $\begin{array}{c}\text { MSC Passage } \\
\text { Number }\end{array}$ \\
\hline \multirow[t]{3}{*}{$\operatorname{Dog} 1$} & 4.3 & $7.44 \times 10^{5}$ & IVT & & 5 \\
\hline & 4.3 & $4.10 \times 10^{6}$ & $\mathrm{CM}$ & $25 \%$ TPP $1+$ GFP & 5 \\
\hline & 5.3 & $6.49 \times 10^{6}$ & ICV & $25 \%$ TPP1 + GFP & 5 \\
\hline \multirow[t]{4}{*}{$\operatorname{Dog} 2$} & 4 & $3.45 \times 10^{6}$ & IVT & & 5 \\
\hline & 4.7 & $1.60 \times 10^{7}$ & ICV & $25 \%$ TPP $1+$ GFP & 7 \\
\hline & 5.4 & $1.34 \times 10^{6}$ & IVT & & 5 \\
\hline & 5.7 & $1.30 \times 10^{7}$ & ICV & $\begin{array}{c}25 \% \text { TPP } 1+ \\
\text { mCherry }\end{array}$ & 6 \\
\hline \multirow[t]{4}{*}{$\operatorname{Dog} 3$} & 3.7 & $7.61 \times 10^{6}$ & IVT & & 6 \\
\hline & 4.2 & $1.50 \times 10^{7}$ & ICV & $25 \%$ TPP $1+$ GFP & 14 \\
\hline & 4.8 & $1.84 \times 10^{6}$ & IVT & & 5 \\
\hline & 5.4 & $1.72 \times 10^{7}$ & ICV & $\begin{array}{c}25 \% \text { TPP1 + } \\
\text { mCherry }\end{array}$ & 10 \\
\hline \multirow[t]{2}{*}{ Dog 4} & 5.5 & $2.53 \times 10^{6}$ & IVT & & 4 \\
\hline & 6.1 & $8.12 \times 10^{6}$ & ICV & $25 \%$ TPP $1+$ GFP & 7 \\
\hline \multirow[t]{2}{*}{ Dog 5} & 4 & $5.38 \times 10^{6}$ & $\mathrm{CM}$ & TPP1 & 5 \\
\hline & 4.9 & $1.10 \times 10^{7}$ & $\mathrm{CM}$ & TPP1 & 5 \\
\hline \multirow[t]{2}{*}{ Dog 6} & 3.5 & $2.58 \times 10^{6}$ & IVT & & 2 \\
\hline & 8.3 & $1.00 \times 10^{7}$ & $\mathrm{CM}$ & mCherry & 3 \\
\hline Dog 7 & 3.7 & $1.39 \times 10^{6}$ & IVT & & 2 \\
\hline $\operatorname{Dog} 8$ & 5 & $2.05 \times 10^{7}$ & $\mathrm{CM}$ & mCherry & 3 \\
\hline \multirow[t]{2}{*}{$\operatorname{Dog} 9$} & 3.8 & $1.62 \times 10^{6}$ & IVT & & 2 \\
\hline & 5 & $2.27 \times 10^{7}$ & $\mathrm{CM}$ & TPP1 & 3 \\
\hline
\end{tabular}

Table 3-1: Treatment details for MSC injections. Details for IVT injections of TPP1-MSCs in the left eyes are shown. Contralateral eyes received GFP-MSCs of the same passage number, and additional details for control eyes including number of cells injected can be found in Appendix A. AAV vectors were used to deliver the transgene. In some cases, a portion of TPP1-MSCs were transduced to also express a fluorescent marker (i.e., 25\% TPP1 \& GFP and 75\% TPP1 only). IVT = intravitreal, $\mathrm{CM}=$ injection at cisterna magna, ICV = intracerebroventricular injection

\subsubsection{Intravitreal Injection of MSCs}

The dog was dilated, sedated and anesthetized and ERG and SLO/OCT procedures were performed prior to injection. IOP was measured following SLO/OCT imaging. Then the ocular surface was cleaned with dilute (1:50) povidone-iodine solution and 1 drop of ofloxacin and 1 drop of proparacaine were 
administered to each eye. The cell suspensions were injected using a $1 \mathrm{~mL}$ insulin syringe with a 0.5 inch 27 -gauge attached needle. After gently mixing the suspension by inverting the tube, the suspension was drawn into the syringe. The dorsolateral bulbar conjunctiva was grasped with forceps and the globe rotated ventromedially. The needle was then inserted from the top of the eye 5-7 $\mathrm{mm}$ posterior to the limbus with needle trajectory pointed towards the optic nerve. Immediately after injection, SLO/OCT imaging was repeated to visualize the injected cells. IOP was rechecked 20 minutes after the injection and repeated if necessary, until IOP normalized.

\subsubsection{Intrathecal (Cisterna Magna) Injection of MSCs}

The dog was placed under general anesthesia following a sedation and induction protocol of $20 \mu \mathrm{g} / \mathrm{kg}$ IM dexmedetomidine, $0.015 \mathrm{mg} / \mathrm{kg}$ IM or IV Buprenorphine and IV propofol to effect. General anesthesia was maintained with isoflurane, and the dog was maintained on a ventilator. The dog was placed in right lateral recumbency and the hair clipped from the dorsum of the neck from C3 to just past the occipital protuberance. The shaved area was prepared with chlorhexidine scrub and solution in a sterile technique. The cells were mixed by repeated inversion of the tube, or by repeatedly gently drawing them up into and expelling them from the injection syringe if necessary. A small volume of air followed by the cell suspension was then drawn up into a $3 \mathrm{~mL}$ syringe using a 22-guage 1 inch needle and $1 \mathrm{~mL}$ of the suspension was injected into the cerebellomedullary (CM) cistern using a 22-gauge 1.5 inch spinal needle over a period of one minute. The dog was then placed in sternal recumbency and on an 
incline whereby the head was at the lowest point on the tilt; this was maintained for a minimum of 15 minutes and no more than 45 minutes. A full dose of atipamezole was then given IM. Buprenorphine and Tramadol were used for pain control as needed.

\subsubsection{Intracerebroventricular Injection of MSCs}

Two weeks prior to intracerebroventricular (ICV) injection, a head computerized tomography (CT) scan without contrast was performed and used for 3D printing of a fiducial array.

On the day of the injection, the dog was anesthetized, placed in sternal recumbency, and the head was stabilized with tape. The dog was surgically prepared with diluted betadine. Sterile drapes were placed over the surgical site, and a midline incision using a \#11 blade was made at the midline from the frontal lobe to the caudal rostrum. Cautery was used for hemostasis. At the surgical site the periostium was lifted with a periosteal freer. The skull implant was stabilized in place using four $2.2 \mathrm{~mm}$ self-tapping titanium screws placed using a Dewalt power screwdriver. Four fiducial pedals were placed using super glue. A head CT was performed and the images were uploaded to Brainsight to choose and configure the targets, the left and right lateral ventricles.

The dog was again placed in sternal recumbency and surgically prepared with dilute betadine and sterile drapes. $20 \mathrm{mg} / \mathrm{kg}$ of Cephazolin reconstituted to $100 \mathrm{mg} / \mathrm{mL}$ was given intravenously and repeated every 90 minutes. Three surgical stab incisions were made using a \#11 blade, two at the cranial 
zygomatic arch and masticatory muscles and a third at the suboccipital protuberance. The surgical arm was set in place with the C-arm affixed to the dog at the three points where surgical stab incisions were made. Registration and validation of tracker positions for the left and right ventricles was performed using Brainsight. The gross and fine surgical arm were attached and measurements for targeting the left ventricle were rechecked. Using the \#11 blade, a longitudinal incision was made caudal and slightly lateral to the point of insertion for the left lateral ventricle. The temporalis muscle was elevated and the parietal bone exposed using Gelpie retractors. A pneumatic Hall Air Drill was used to perform a craniotomy, with frequent lavages with sterile saline. Cautery was used for hemostasis. Trackers were then re-registered to ensure accuracy and a T-Base with the caliper and "hockey stick" were reregistered and calibrated. A $1 \mathrm{~mL}$ Hamilton glass syringe with a 6 inch 22-guage Hamilton needle was mounted onto the caliper apparatus and the Hamilton syringe filled with sterile saline. The Hamilton needle was then advanced into the ventricles with a distance calculated by Brainsight. The plunger of the syringe was pulled back to check for CSF to confirm that the needle entered the ventricle. An additional $1 \mathrm{ml}$ Hamilton glass syringe and 6 inch, 22-guage needle was used to draw up the cell suspension and placed in the caliper apparatus. The cell suspension was injected into the left ventricle through the syringe. A saline rinse was performed using the same syringe. The Hamilton needle and caliper apparatus were removed from the left ventricle and the surgical site was lavaged with sterile saline and covered with gel foam. A cruciate screwdriver was used to 
remove the skull implant fiducial array. 3-0 polydioxanone suture (Ethicon, Inc., Somerville, $\mathrm{NJ}$ ) was used to close the temporalis muscle, fascia and subcutaneous layer in a simple continuous pattern. The skin was closed using a simple interrupted pattern with 3-0 Ethilon suture (Ethicon, Inc., Somerville, NJ). Buprenorphine and Tramadol were used for pain control as needed following the procedure.

\subsubsection{Immunosuppressants and Inflammation Control}

One to two weeks prior to the first injection (IVT, ICV or CM), administration of cyclosporine and leflunomide $(2.5 \mathrm{mg} / \mathrm{kg}$, once daily) was initiated for dogs 1-5. $25 \mathrm{mg}$ cyclosporine was given twice daily until the dog reached a weight of $4 \mathrm{~kg}$, at which point the dose was increased to $35 \mathrm{mg}$. Dogs were maintained on cyclosporine continuously, except dog 2 for whom cyclosporine was discontinued at 9 months of age. Because inflammation in previous MSC-treated dogs had been mild if it occurred at all, dogs 6-9 received neither cyclosporine nor leflunomide.

For dogs receiving intravitreal MSCs, twice daily prednisolone acetate $1 \%$ ophthalmic suspension and once daily tropicamide $1 \%$ ophthalmic solution (dogs 1-4 only) as well as four times daily Durezol and three times daily Nevenac drops (all dogs that received IVT MSCs) were initiated 24 hours before injection. Immediately following the injection, dogs were given a sub-Tenon's injection of 2 $\mathrm{mg}$ triamcinolone acetonide in both eyes. All drops were discontinued by one week post-treatment if there was no evidence of intraocular inflammation. Some

dogs developed a mild transient uveitis which was treated with additional topical 
medications (prednisolone acetate, ketorolac, flurbiprofen, tropicamide or carprofen) administered as needed and discontinued when inflammation resolved.

For dogs 1-4 receiving MSCs in the CSF via CM or ICV injection, $1 \mathrm{mg} / \mathrm{kg}$ oral prednisone was initiated the day before the injection. This dose was maintained for $3-4$ days, tapered $0.5 \mathrm{mg} / \mathrm{kg}$ for $3-4$ days, then discontinued if no signs of inflammation were noted.

\subsubsection{In vivo Measures to Assess Retinal Function and Structure}

Retinal function was assessed with bilateral ERG recordings, performed monthly following a baseline pre-treatment measurement, as described in 2.2.5. When necessary, midazolam $(0.25 \mathrm{mg} / \mathrm{kg})$ was used to provide additional sedation during the procedures. The presence of injected cells and the fluorescence of GFP-MSCs were confirmed in vivo with SLO and OCT imaging immediately following injection. Retinal structure, including CMR lesion development, was assessed with SLO/OCT imaging performed at baseline prior to MSC injection and then monthly thereafter as described in 2.2.6.

\subsubsection{MRI and Ventricular Volume Analysis}

Brain magnetic resonance (MR) imaging was performed on a monthly basis starting at approximately 4 months of age for dogs that received CNS MSCs (dogs 1-5, 8 and 9). All imaging was performed under general anesthesia using a 3.0T Toshiba/Canon Titan scanner (Canon Medical Systems USA, 
Tustin, CA) and an orthopedic multichannel speeder coil. Images were obtained using the following parameters: localizer, T2-weighted sagittal (T2W: TR 3028$3316 \mathrm{~ms}$, TE $120 \mathrm{~ms}$, slice thickness $2.5 \mathrm{~mm}$, slice gap $2.8 \mathrm{~mm}$, FOV 576-784 x 640-800 mm), T2W coronal (TR 3028-3461 ms, TE 120 ms, slice thickness 2.5 mm, slice gap 2.8 mm, FOV 576-768 x 640-940 mm), T2W transverse (TR 68378302 ms, TE 120 ms, slice thickness 3 mm, slice gap 3.3 mm, FOV 512-768 x 512-704 mm), and MPRAGE (TR $6.8 \mathrm{~ms}$, TE $2.7 \mathrm{~ms}$, slice thickness $1 \mathrm{~mm}$, slice gap $0.5 \mathrm{~mm}$, FOV 400x420 mm). Quantification of ventricular volume was done in Brainsight (Rogue Research, Montreal, Quebec) on the MPRAGE sequence images. MR images were thresholded to isolate the ventricles. Seeds which propagated to subsequent images were placed in the thresholded ventricle of the most rostral or caudal slice containing ventricle. The software automatically tracked the seeds in subsequent slices, and manual adjustments were made to ensure that the entire ventricular system was accurately selected. The selected area was used by the program to calculate the total area of the ventricles in $\mathrm{mm}^{3}$.

\subsubsection{Cognitive Testing}

A serial reversal learning task conducted with a T-maze apparatus (CanCog Technologies, Toronto, Onatrio, Canada) was used to assess memory and learning (Sanders et al., 2011). The T-maze consisted of a start box, runway and two reward arms. Between 2-4 months of age, dogs were slowly acclimatized to the room, apparatus and testing procedures. Dogs were initially allowed to freely explore the room, then trained to explore the T-maze for food rewards, and then food rewards were placed only in both reward arms. 
Data collection time points were repeated monthly starting at 4 months of age. Dogs were not tested during the 12 hour period after sedation or during the 24 hour period after anesthesia. Each data collection time point consisted of five phases: preference determination, preference reinforcement and three reversal learning phases. During the preference determination phase, food rewards were placed in both reward arms and the dog's preferred side for that time point was determined as the side to which the dog went most often over nine trials in a single session. During preference reinforcement, food was placed only in the preferred reward arm and sessions were repeated daily until the dog chose the preferred arm 9/10 times during one session or $8 / 10$ times during two consecutive sessions. These were followed by three reversal learning phases. The food reward was placed in the non-preferred reward arm during the first phase, the preferred arm during the second, and the non-preferred arm during the third. The dog was required to choose the correct arm 9/10 times during one session or $8 / 10$ times during two consecutive sessions to progress to the next phase. The number of incorrect choices made prior to reaching this criterion was recorded, and the average number of incorrect choices for the three phases was used for data analysis.

\subsubsection{Euthanasia and Necropsy}

Dogs were euthanized at end-stage neurological disease as described in 2.2.7 following final ERG and retinal imaging procedures. Retinas were collected from all dogs and processed as described in 2.2.7, except that the retinas were fixed in either $2.5 \%$ glutaraldehyde fixative or immuno fixative $(3.5 \%$ 
paraformaldehyde, $0.05 \%$ glutaraldehyde, $0.12 \mathrm{M}$ sodium cacodylate, $1 \mathrm{mM}$ $\mathrm{CaCl}_{2}, \mathrm{pH}$ 7.4). Vitreous was collected from dogs 6-9.

After processing the eyes, the brain was collected from all dogs except dogs 3 and 4 . The calvarium was first removed. For dogs 6-9, a needle was inserted into the lateral ventricles and CSF was withdrawn into an attached $1 \mathrm{~mL}$ syringe. The brain was gently removed and chilled in ice-cold Plasmalyte (Baxter International, Deerfield, IL) for at least 10 minutes. Using a pre-chilled adult Rhesus monkey brain matrix (Electron Microscopy Sciences, Hatfield, PA), the brain was sliced into $4 \mathrm{~mm}$ coronal slices. Slices were either frozen on dry ice or fixed in immuno fixative, $2.5 \%$ glutaraldehyde fixative or EM fixative $(2.0 \%$ glutaraldehyde, $1.12 \%$ paraformaldehyde, $0.13 \mathrm{M}$ sodium cacodylate, $1 \mathrm{mM}$ $\mathrm{CaCl}_{2}, \mathrm{pH}$ 7.4). In some cases, slices were bisected along the midline and each half fixed or frozen separately.

The vitreous of dogs 6,7 and 9 and the CSF of dogs 6,8 and 9 were examined for the presence of MSCs. For dogs 6 and 7, as much of the vitreous as possible was dissected out as described in 2.2.7. The vitreous from the GFPMSC eye was transferred to a small petri dish and examined with a fluorescence stereoscope. Images of any fluorescence were captured. The entire vitreous from the left and right eyes was placed in separate $15 \mathrm{~mL}$ conical tubes, brought to 3 $\mathrm{mL}$ with $1 \mathrm{X}$ PBS and homogenized by pulsing. CSF collected from the lateral ventricles during necropsy and CSF collected from the cerebromedullary cistern just prior to euthanasia were processed separately. CSF and homogenized vitreous samples were centrifuged for 10 minutes at $1545 \mathrm{xg}$. If a pellet was 
observed, the supernatant was stored at $-80^{\circ} \mathrm{C}$ and the pellet was resuspended in $100 \mu \mathrm{L}$ of $0.17 \mathrm{M}$ cacodylate buffer. If no pellet was observed, the homogenate was gently mixed. $10 \mu \mathrm{L}$ of either solution was added to $200 \mu \mathrm{L}$ cacodylate buffer and a Scepter cell count was performed. $5 \mu \mathrm{L}$ of the diluted solution was spotted on a microscope slide, mounted with Vectashield Antifade Mounting Medium with DAPI (Vector Laboratories, Burlingame, CA), and examined with phase contrast and fluorescence microscopy. For dog 9, the vitreous from the GFP-MSC eye was fixed in immuno fixative overnight and examined with a fluorescence stereoscope and GFP filter set.

\subsubsection{Statistical Analysis}

All statistical tests were performed using SigmaPlot (Systat Software Inc., San Jose, CA). Data were subjected to the Shapiro-Wilk test to confirm normal distribution. Repeated measures 2-way ANOVA was used to compare ERG data from CLN2-affected eyes treated with TPP1-MSCs to those eyes treated with GFP-MSCs to determine if the treatment was able to prevent deficits in ERG bwave amplitudes related to CLN2 disease progression. Follow-up pairwise comparisons were performed with the Holm-Sidak correction $(\alpha=0.05)$ to control family-wise error rate. Data from treated dogs were grouped into four age groups: 4 months (3-4.9), 6 months (5-6.9), 8 months (7-8.9) and 10 months (9-10.9) of age. The average amplitude was used when a dog had more than recording in an age range. 


\subsection{Results}

\subsubsection{Detection of MSCs after Transplantation}

MSCs were visible with SLO/OCT imaging and fundoscopy in both eyes immediately after injection as a large mass which dissipated quickly and usually could not be visualized by two weeks post-injection. MSCs could not be detected in the vitreous of either eye post-mortem in dogs 6 or 7 . The cells dispersed as expected in dog 9 following injection and were not visible at 6 months of age; however, at 8 and 10.5 months of age, material was visible in the vitreous of both eyes (Figure 3-1A and B). In the GFP-MSC eye, this material was fluorescent (Figure 3-1A). Dog 9 was euthanized following SLO/OCT imaging at 10.5 months of age. The vitreous of the GFP-MSC eye was examined post-mortem with a fluorescence stereoscope and GFP filter set, and many fluorescent cells were visible (Figure 3-1C). Material that may be TPP1-MSCs was visible in the TPP1MSC eye at 8 and 10 months of age (Figure 3-1B) but we have not yet examined the vitreous of this eye post-mortem. MSCs could not be detected in the CSF of dogs 6,8 or 9 . 

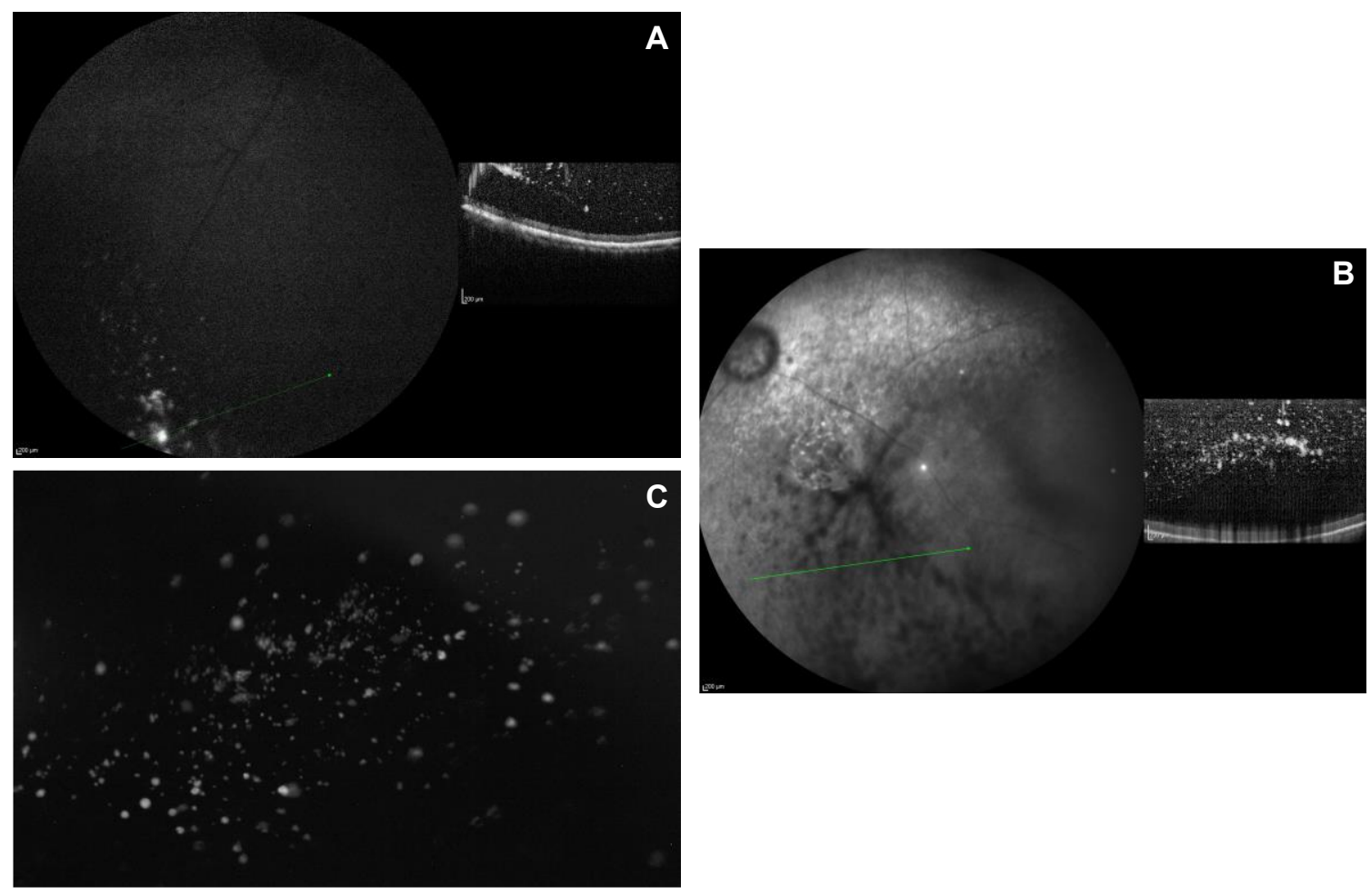

Figure 3-1: A fluorescence SLO/OCT image of material in the GFP-MSC eye (A) and an SLO/OCT image of material in the TPP1-MSC eye (B) of dog 9 at 8 months of age. A fluorescence stereoscope image of the vitreous from the GFPMSC eye of this dog who was euthanized at 10.5 months of age (C).

\subsubsection{Retinal Function}

A previous study found that intravitreal administration of TPP1-MSCs partially preserved retinal function and structure through 10 months of age (Tracy et al., 2016). Those results were replicated in the present study. While b-wave amplitudes declined over time in both treated and control eyes, mixed rod and cone amplitudes remained significantly larger in TPP1-MSC treated eyes than GFP-MSC control eyes through 10 months of age (Figure 3-2A). Pure rod response b-wave amplitudes were significantly larger in treated eyes than control eyes at 6 and 8 months of age, but by 10 months of age amplitudes in both 
treated and control eyes were similarly reduced from normal (Figure 3-2B).

Treatment did not preserve cone response amplitudes (Figure 3-2C and D).
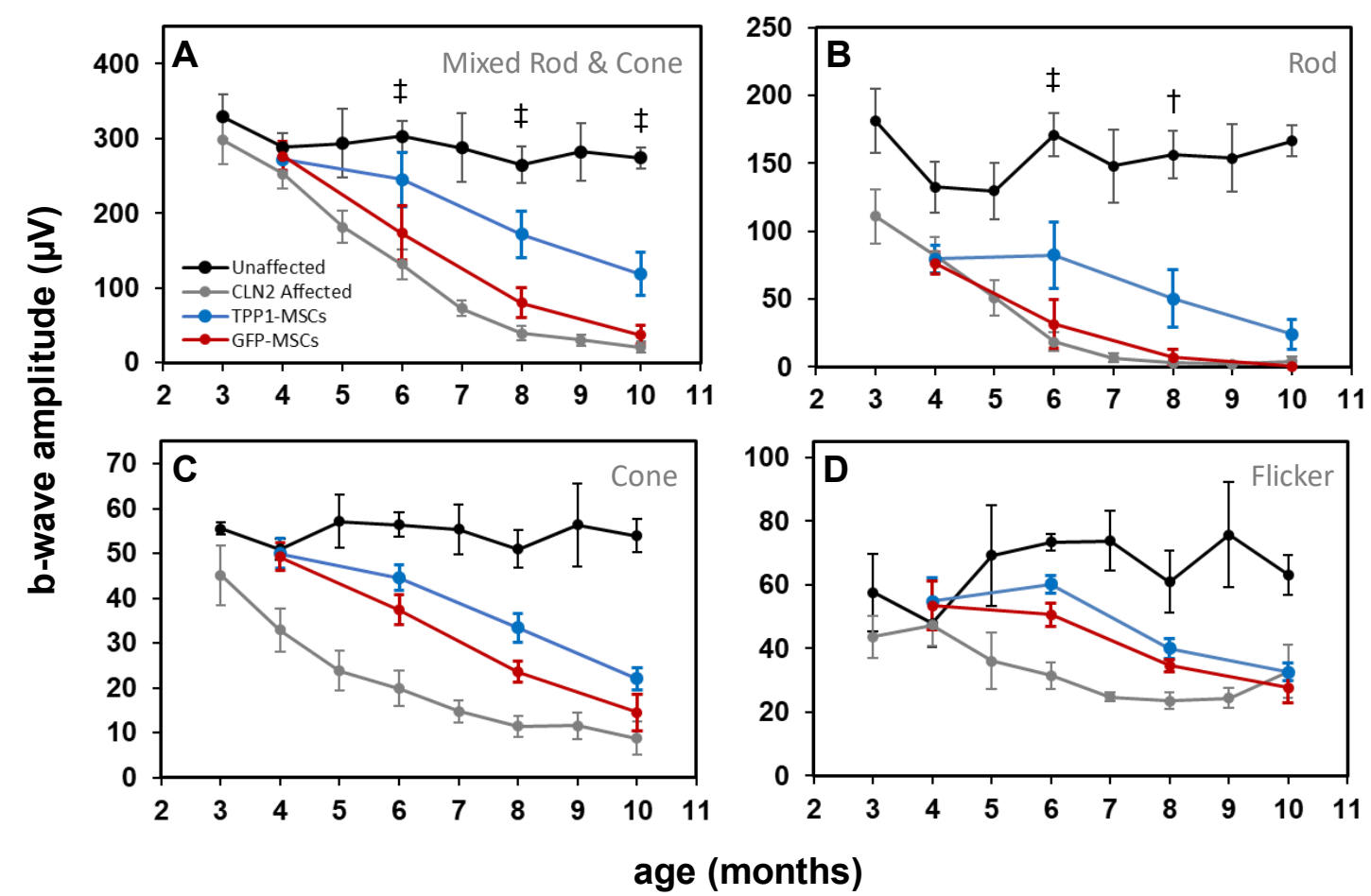

Figure 3-2: Mean mixed rod and cone (A), pure rod (B), pure cone (C) and cone flicker (D) b-wave amplitudes from dogs that received intravitreal TPP1-MSCs (blue line, $\pm S E M, n=6$ ) in one eye and GFP-MSCs (red line, $\pm S E M, n=6$ ) in the contralateral eye. Black and gray traces represent historical data from normal $(n=7)$ and CLN2-affected $(n=6)$ untreated dogs from the same research colony $( \pm$ SEM). $\neq p \leq 0.001, \uparrow p=0.003$.

While treatment had only a modest effect overall, one dog experienced a pronounced treatment benefit in the TPP1-MSC eye. Dog 6 had a marked increase in mixed rod and cone, pure rod and pure cone b-wave amplitudes in the TPP1-MSC eye following treatment (Figure 3-3). These amplitudes remained normal or greater than normal through 7 months of age then began to decline. Mixed rod and cone responses remained normal through 9 months of age. We 
were able to follow this dog to almost 12 months of age, and even at this age mixed rod and cone and pure rod b-wave amplitudes were partially preserved. These results were mirrored in the GFP-MSC eye though to a lesser degree. Surprisingly, dog 6's mixed rod and cone amplitudes remained in the normal range in the GFP-MSC eye through 7 months of age. Mixed rod and cone, pure rod and pure cone b-wave amplitudes in the GFP-MSC eye of this dog increased following treatment and remained elevated through 7 months of age before sharply declining.

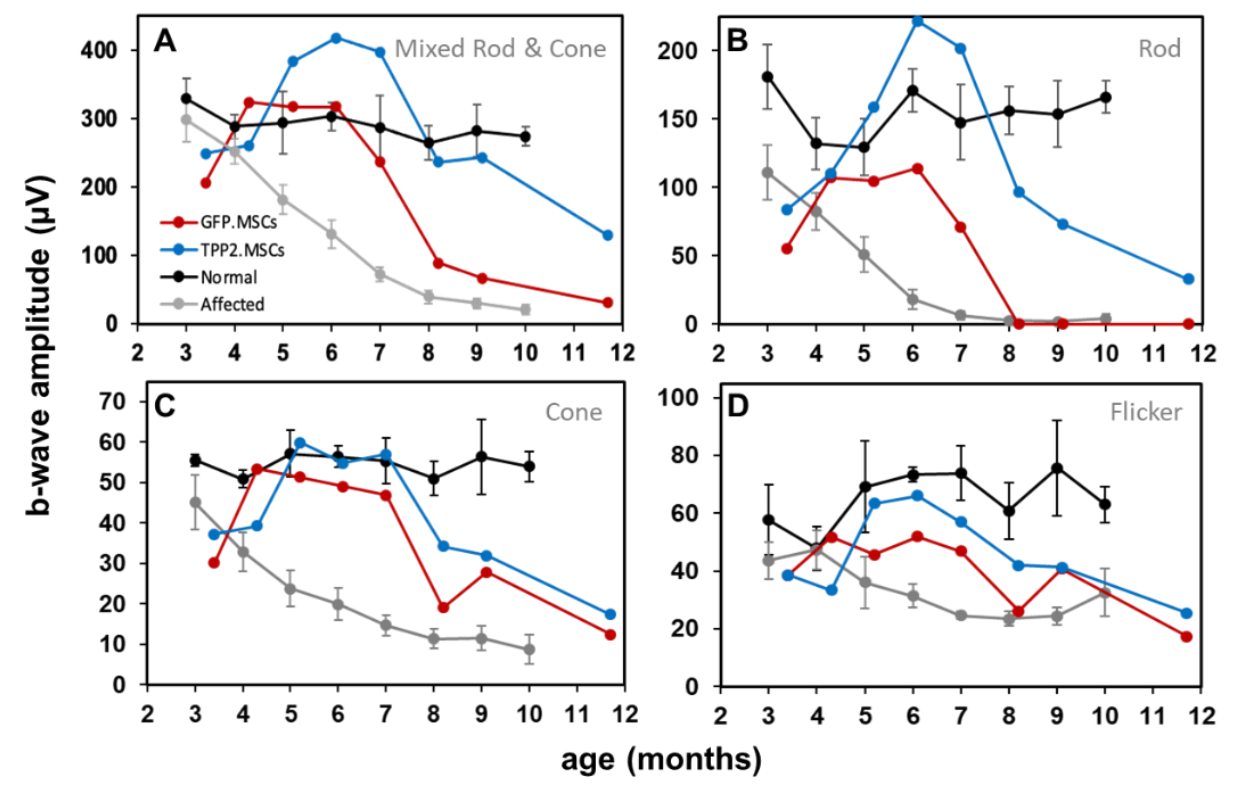

Figure 3-3: Mixed rod and cone (A), pure rod (B), pure cone (C) and cone flicker (D) b-wave amplitudes from dog 6 that received intravitreal TPP1-MSCs in one eye and GFP-MSCs in the contralateral eye.

\subsubsection{Lifespan}

TPP1-MSC treatment did not extend lifespan (Figure 3-4). Dogs treated with CSF infusions of TPP1-MSCs were humanely euthanized between 43 and 
45 weeks of age due to progression of neurological disease symptoms.

Untreated affected dogs were euthanized between 39 and 47 weeks of age. The longest-lived dog in this study, who lived to 51 weeks (11.7 months) of age, received mCherry-MSCs.

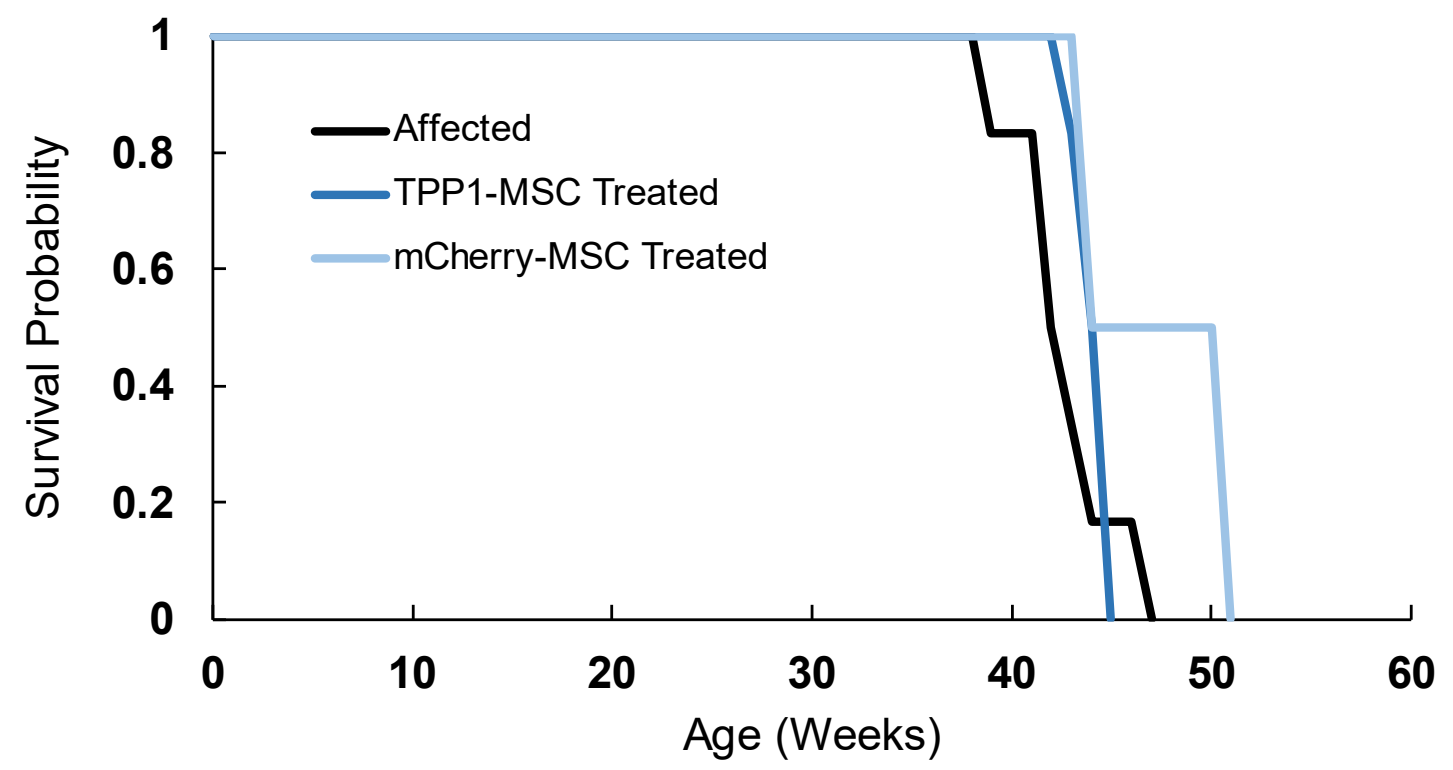

Figure 3-4: Kaplan-Meier Curve showing survival probability of untreated affected $(n=6)$ dogs and dogs who received CSF infusions of TPP1-MSCs $(n=6)$ and mCherry-MSCs $(n=2)$.

\subsubsection{Onset of Neurologic Disease Signs}

There were no apparent delays in the onset of neurologic disease signs in dogs that received CSF infusions of MSCs (Figure 3-5). 
Onset of Neurologic Disease Signs

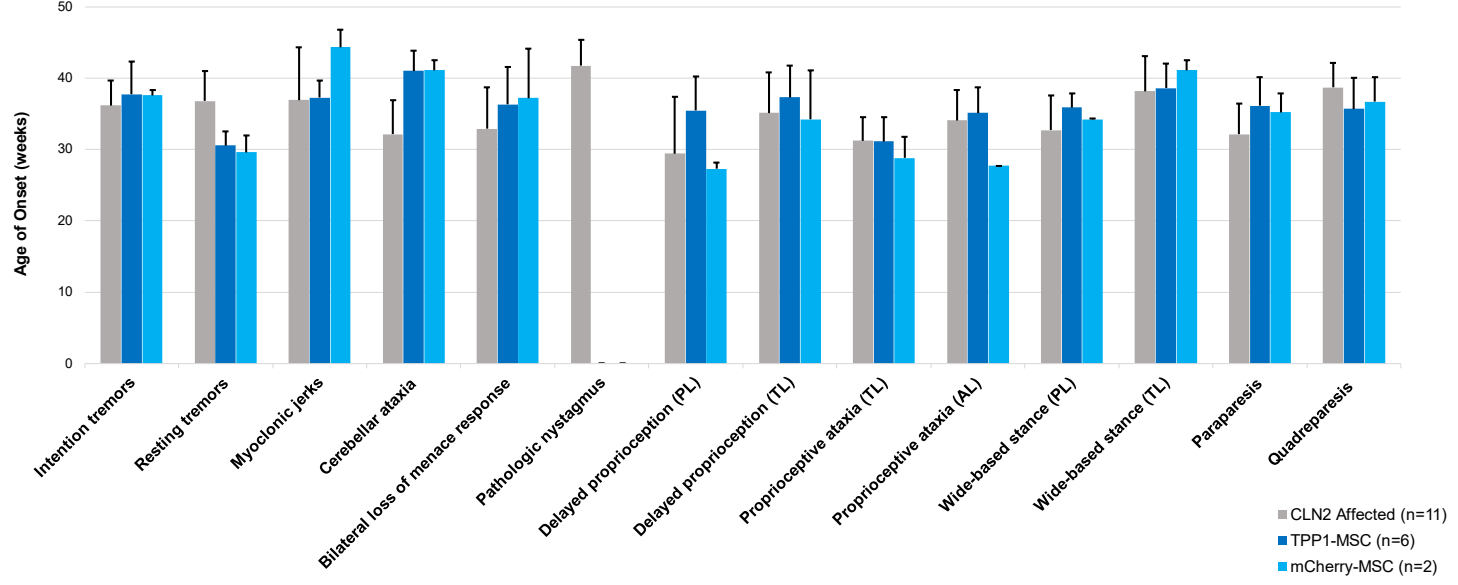

Figure 3-5: Onset of neurologic disease signs + standard deviation in untreated dogs $(n=11)$ and in dogs that received CSF infusions of TPP1-MSCs $(n=6)$ and mCherry-MSCs $(n=2)$. No MSC-treated dogs developed pathologic nystagmus. $\mathrm{PL}=$ pelvic limbs; $\mathrm{TL}=$ thoracic limbs; $\mathrm{AL}=$ all limbs

\subsubsection{Cognitive Ability}

CSF infusion of MSCs did not improve cognitive performance as assessed by the T-maze. After 6 months of age, MSC-treated dogs' average performance on the task progressively worsened, and no dog was able to complete the task after 9 months of age (Figure 3-6). However, prior to this point dog 9 did appear to experience improved performance on the task relative to untreated as well as the other TPP1-MSC treated CLN2-affected dogs (Figure 3-6D). This dog made similar numbers of errors from 5 to 8 months of age, only worsening at 9 months. 
A

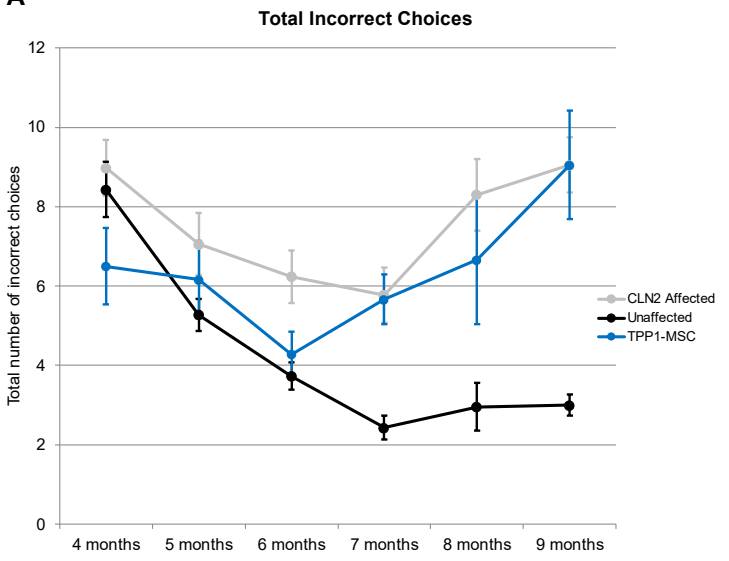

C

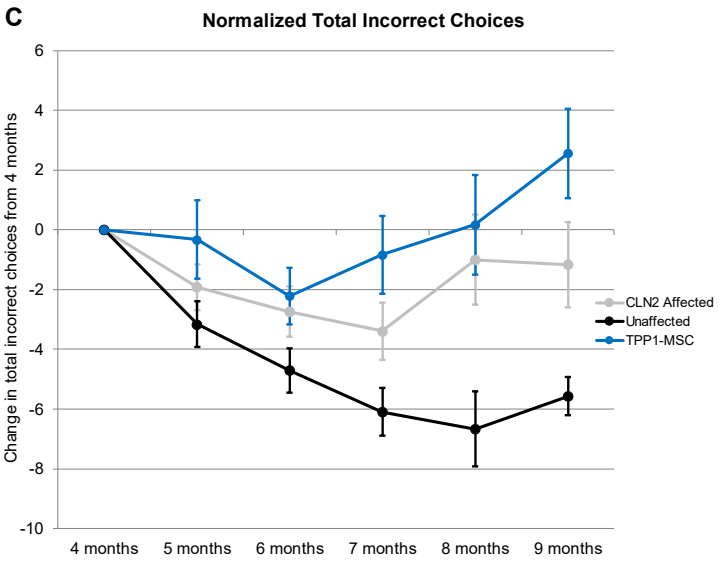

B
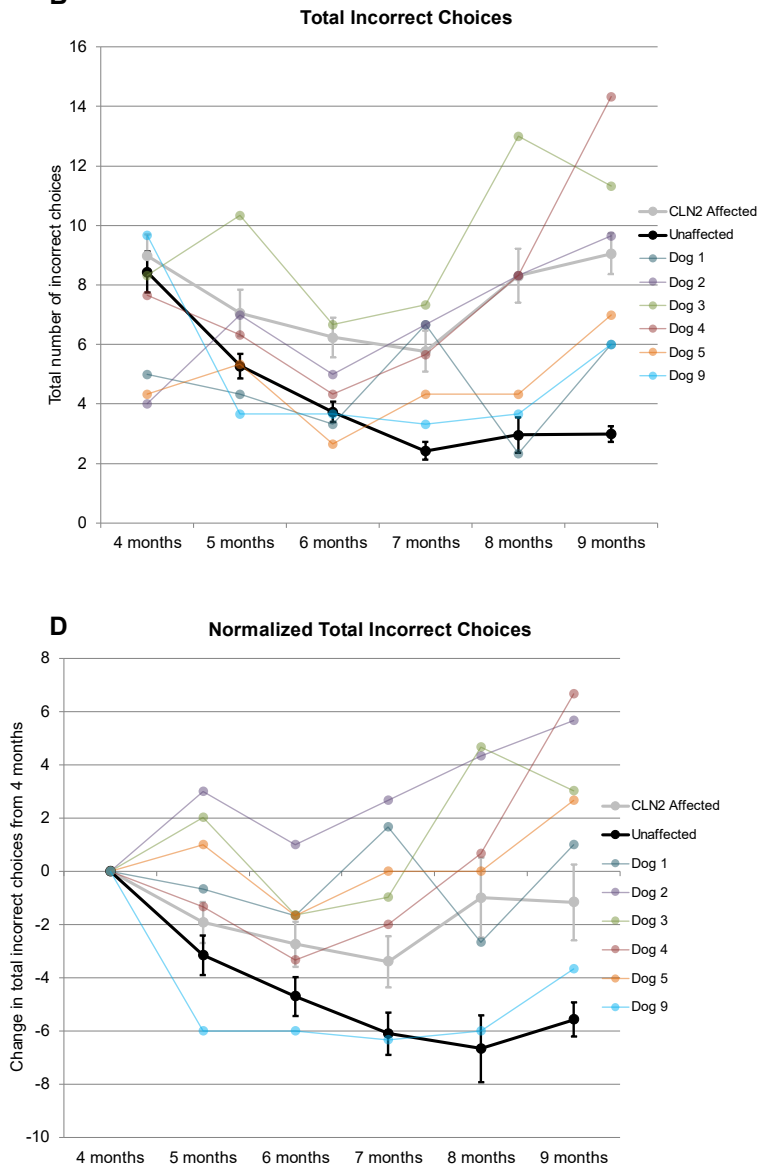

Figure 3-6: Total incorrect choices (A) and change in total incorrect choices from 4 months of age $(C)$ for monthly T-maze assessment for unaffected (black line, $\mathrm{n}=17, \pm$ SEM), untreated CLN2-affected (grey line, $\mathrm{n}=17, \pm$ SEM) and TPP1-MSC treated (blue line, $n=6, \pm S E M$ ) dogs. Individual total incorrect choices of TPP1MSC treated dogs (B) and change in total incorrect choices (D) are also shown.

\subsubsection{Brain Atrophy}

CSF infusion of MSCs did not prevent generalized brain atrophy. Total ventricular volume increased dramatically in MSC-treated dogs (Figure 3-7). Enlarged ventricles and reduced cortical grey matter were apparent on MR images (Figure 3-8). 


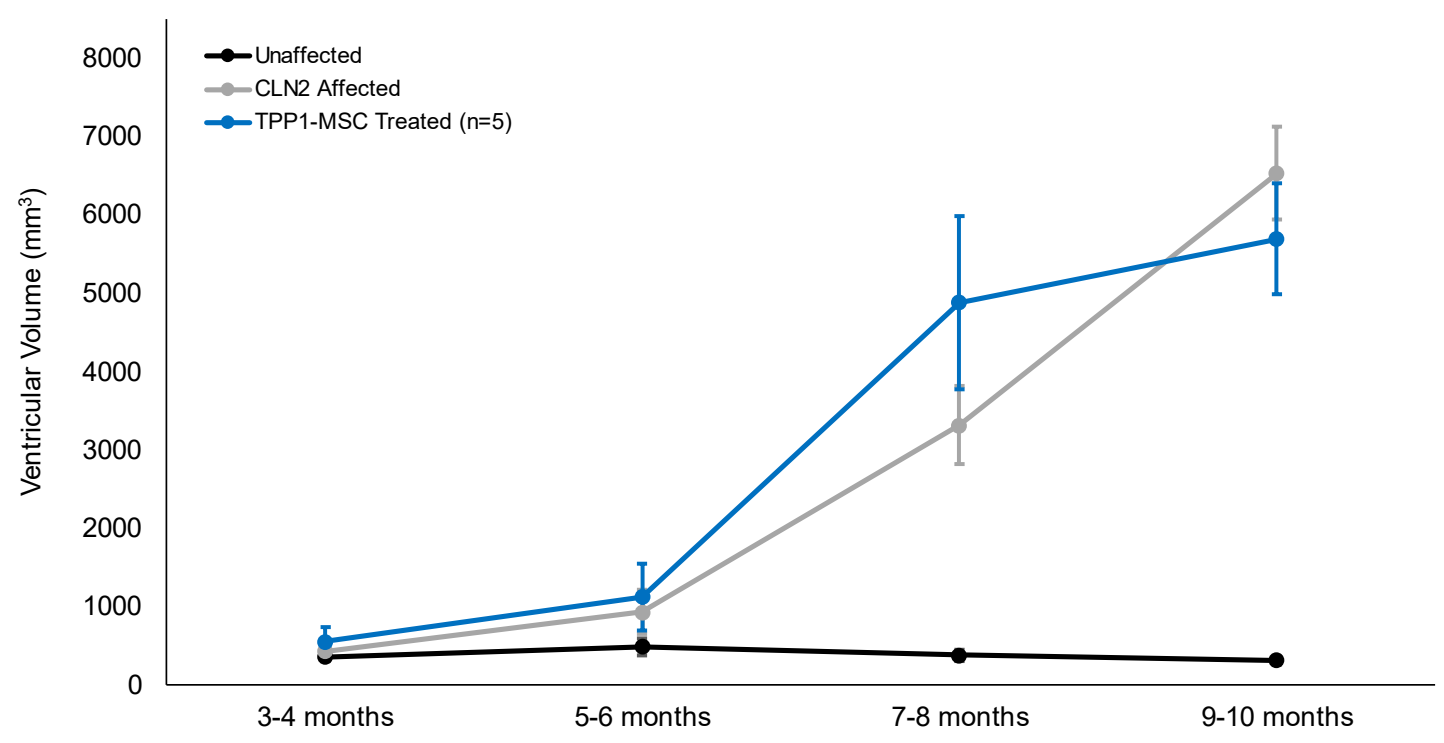

Figure 3-7: Ventricular volume over time in unaffected (black line, $n=5, \pm S E M)$, untreated CLN2-affected (grey line, $n=8, \pm S E M$ ) and TPP1-MSC treated (blue line, $n=6, \pm S E M)$ dogs.

\section{5 months $\quad 9.5$ months}

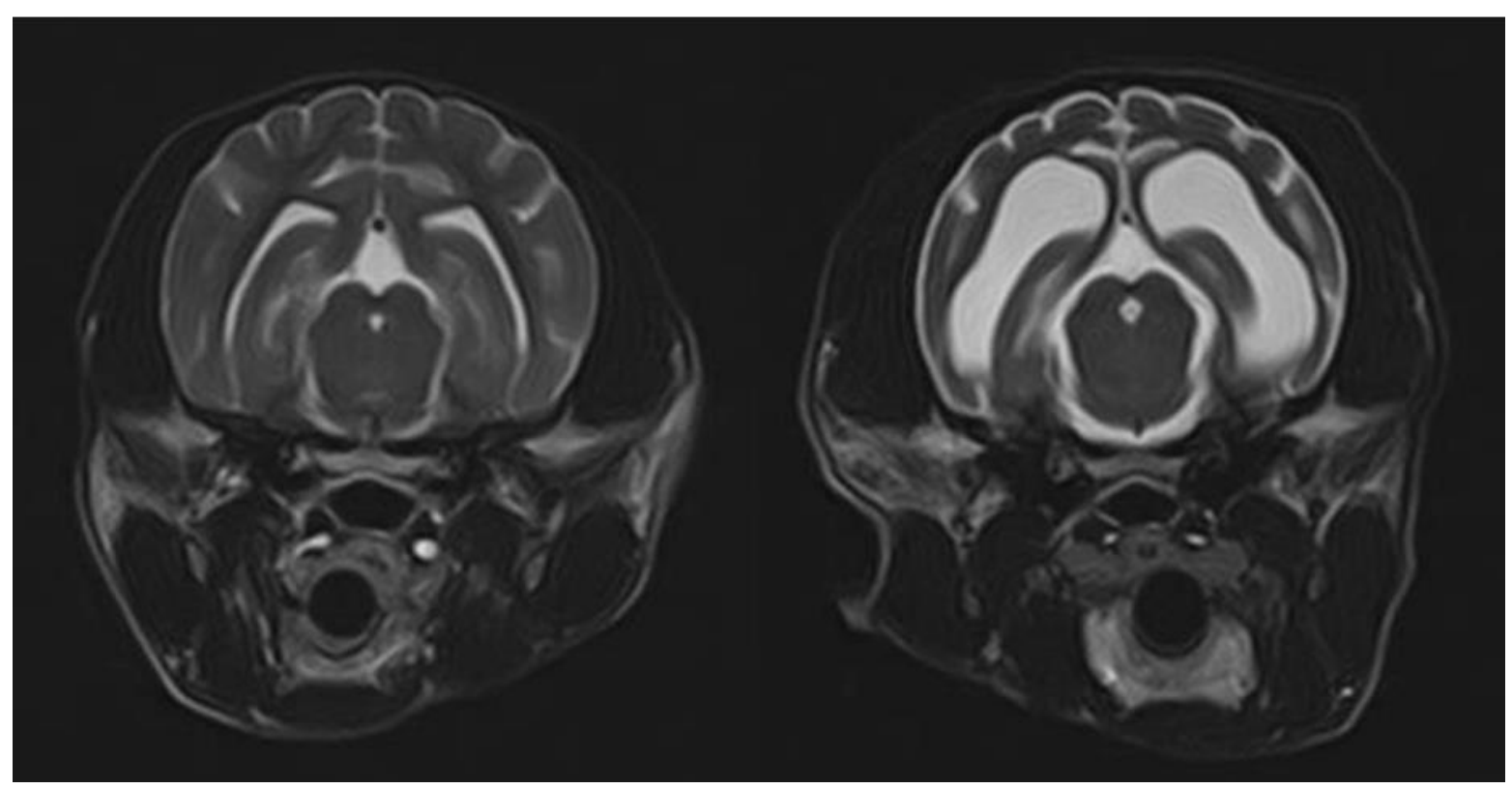

Figure 3-8: MR images from dog 9, who received a CSF infusion of TPP1MSCs, at 3.5 and 9.5 months of age. Enlarged ventricles and reduced cortical grey matter are apparent at 9.5 months of age. 


\subsection{Discussion}

Intravitreal injection and CSF infusion of MSCs appears to be safe and welltolerated. Some dogs that received eye treatments exhibited mild transient uveitis within the first week after treatment, but this resolved within 1 to 4 weeks with no lasting negative effects in any case. No dog that received a CSF injection of cells exhibited a clinical inflammatory response. Dogs $6-9$ were on no immunosuppressive drugs or anti-inflammatory medications after the first week. In contrast, brain and eye enzyme replacement and gene therapies tested in this model resulted in significant inflammatory responses directed against the TPP1 protein even with anti-inflammatory treatment. This may be due to the innate immunosuppressive properties of MSCs and/or to the slow release of small amounts of hTPP1 from MSCs. However, we have not yet been able to locate TPP1-MSCs in the brain or eye following injection and cannot confirm that they survive and continue to secrete hTPP1 throughout the study period.

In the eyes, partial preservation of ERG amplitudes through 10 months of age in TPP1-MSCs treated eyes seems to suggest that the cells survive and secrete hTPP1 for at least several months. Going forward, it will be important to try to locate TPP1-MSCs in both the brain and eye after treatment to assess whether they are still present and secreting hTPP1. Additionally, TPP1 immunolabeling in target tissues and detection in the vitreous and CSF will indicate whether hTPP1 is present and reaching the brain and retina, regardless of our ability to locate the transplanted cells. MSCs are known to home to sites of inflammation, so we anticipate that the cells may have migrated towards the 
retina and the surface of the brain but have not confirmed this. Typically, MCS cannot be visualized in the eye in vivo by several weeks post-injection as they disperse into clusters too small to observe. However, in the Tracy et al. study, clusters of cells could be visualized in some eyes up to 9 weeks post-injection. In the present study, small clusters of cells remained visible with SLO/OCT in the GFP-MSC control eyes of dog 2 for three months after injection and of dog 4 through the entire study period. MSCs were not visible in either eye of dog 9 except immediately after injection until 8 months of age, when they again became visible. Tracy et al. conducted post-mortem examination of the vitreous and retina of eyes in which GFP-MSCs remained visible between 4 and 9 weeks after injection and found clusters of GFP-expressing MSCs throughout the vitreous. They did not find cells adhered to the posterior lens capsule or the vitreal surface of the retina or integrated into the retina, so at least in these eyes at this early time point the cells remained in the vitreous and had not migrated to the retina. We were unable to locate any MSCs in the homogenized vitreous of dogs 6 or 7 at ten months of age but we did observe MSCs in the vitreous of dog 9 at this same age. We plan to perform TPP1 immunolabeling on the TPP1MSCs from dog 9 to determine if they continued to produce TPP1 at the end of the study period. Therefore, at least in some cases MSCs can survive for long periods of time in the vitreous. It is unclear if the absence of detectable cells in the vitreous of dogs 6 and 7 at ten months of age is because the cells did not survive or because they migrated toward the retina. In future studies, encapsulation of MSCs prior to injection or injection into periocular spaces could 
be considered as alternative approaches to facilitate later identification and retrieval of the cells (Ding et al., 2019; Lee et al., 2012).

So far, we have been unable to find cells in the CSF after injection. We examined the CSF of several dogs and did not observe any MSCs suggesting that the cells may be cleared from the CSF or migrate toward the brain or spinal cord. Studies of administration of MSCs to the CSF in rat and mouse models of amyotrophic lateral sclerosis (ALS) have reported conflicting results, with some showing survival of the cells and migration into the spinal cord 10 to 30 days post-injection while another was unable to locate GFP-MSCs in either the CSF or in the spinal cord or brain 14 days after administration (Boucherie et al., 2009; Forostyak et al., 2014; Habisch et al., 2007; Morita et al., 2008; Zhang et al., 2009). Overall, there is little evidence supporting long-term survival of MSCs after injection into the CSF and it seems likely that MSCs circulating in the CSF do not survive long, although cells that home to the parenchyma may survive for at least a month. Multiple injections will likely be required if the cells do not persist long term (Zhang et al., 2009). Dog 6 who lived approximately one month longer than is typical of CLN2-affected dogs received a CM injection of mCherry-MSCs at 8.3 months of age. This suggests that injection at this later age may have been particularly beneficial and supports future studies testing repeated injections of cells throughout the course of disease rather than only at early stages. In MSCtreated ALS mice, the majority of the cells were found near the cisterna magna and in basal cisterns, so in future studies these locations as well as the dorsal spinal subarachnoid space would be worth examining for the pretense of MSCs 
(Habisch et al., 2007). Searching for MSCs is challenging in the dog, since the subarachnoid space and brain are quite large, but it is certainly feasible to examine specific, pre-determined sites. Additionally, CSF administration of MSCs shortly before euthanasia could indicate whether they can be located in the CNS even shortly after injection. An interesting approach to track cells after implantation that has been used in human patients is to label them with a contrast agent, such as superparamagnetic iron oxide, which may facilitate tracking of the cells with MR imaging (Karussis et al., 2010; Shichinohe et al., 2017).

It may be the case that an alternate delivery method will be necessary for CNS treatment. We chose to test intrathecal and ICV administration, but if the cells do not survive in the CSF or migrate to the brain from the CSF, intraparenchymal administration may be worth testing. While not directly comparable to MSC transplantation, combined subcortical and ICV injection of allogeneic neural stem cells was tested in patients with CLN1 and CLN2 disease (Selden et al., 2013). Evidence of donor cell engraftment was seen in 2 of 3 post-mortem brains $1-2.5$ years after transplantation and the treatment was well-tolerated. With this method, MSCs would likely only exert local effects. More widespread distribution of cells may be achievable with intra-arterial transplantation. Intra-arterial transplantation of MSCs in dogs with naturally occurring degenerative myelopathy was shown to be feasible and safe and resulted in accumulation of MSCs in the brain (Malysz-Cymborska et al., 2021). To place the cells in a location where they can secrete TPP1 into the CSF for 
widespread distribution of the protein, MSCs could also be encapsulated prior to delivery or transplanted via a scaffold which may enhance the viability of the cells after transplantation and could even facilitate removal of the cells after a period of time (Heile and Brinker, 2011; Heile et al., 2009; Lam et al., 2013; Yousefifard et al., 2020).

It will be important to determine MSC hTPP1 secretion rates prior to injection and intravitreal hTPP1 concentrations after TPP1-MSC implantation. Based on the ERT studies we can estimate what concentrations of TPP1 are therapeutic, although lower concentrations of hTPP1 in the vitreous in the MSCtreated eyes are likely to be therapeutic since MSCs are expected to continuously secrete hTPP1 as opposed to the bolus injections administered in the ERT studies. By measuring hTPP1 secretion rates by the MSCs in vitro, we can estimate how many cells would need to be implanted to achieve therapeutic concentrations in the vitreous. Preliminary data on hTPP1 secretion by AAV2.CAG. $h$ TPP1 transduced cells in vitro indicates that it is feasible to implant enough cells to achieve vitreal concentrations of hTPP1 similar to those that were therapeutically effective in the intravitreal ERT studies. However, after implantation, hTPP1 expression by the MSCs may be altered. In addition, intravitreal concentrations of hTPP1 will be determined by the balance between the rate of protein production by the MSCs and uptake by the retina. Therefore, in order to optimize the treatment, it will also be necessary to determine intravitreal concentrations of hTPP1 after MSC injections. This same approach 
could also be used for CNS treatment. Our preliminary data suggests that this should be achievable.

Cell culture conditions impact the growth and phenotype of MSCs, and therefore can be expected to influence the therapeutic effects of these cells after implantation. A major difference between the Tracy et al. study and dogs $1-4$ in this study is the MSC passage number and time spent in culture. In the combined brain and eye treatment, MSCs from dogs $1-4$ were cultured simultaneously for both brain and eye treatments from a single bone marrow aspirate; therefore, all cells were in culture longer and were of a higher passage number in order to obtain enough cells for both injections. MSCs for intravitreal injection for these dogs were harvested at passage $4-6$ and cells for CNS infusion for dogs $1-5$ were harvested at passage 5 or greater. In contrast, cells in the Tracy et al. study were harvested only for eye injections at passage 2 . The cells we cultured from the bone marrow were classified as MSCs based on their adherence to the plastic substrate. However, many studies have shown that these types of crude MSC preparations contain a mixture of cell types (Mareschi et al., 2012; Wilson et al., 2019). The different subtypes of cells proliferate at different rates. Therefore, the cellular composition of the cultures will change with increasing passage number, at least for relatively early passages. We hypothesized that cells that spent less time in culture and were expanded less might be more effective. We therefore began to perform separate bone marrow aspirations for brain and eye treatments and reduced the number of plates harvested for brain injections. For dogs $6-9$, all eye injections were done with passage 2 (P2) 
MSCs and CSF infusions with passage 3 (P3) MCSs. In the eye, it seems possible that lower passage cells may be able to achieve better preservation of retinal function. One of the three dogs treated with P2 IVT MSCs, dog 6, experienced a pronounced if not sustained treatment benefit, although the other two dogs treated with P2 IVT MSCs responded similarly to the four P5+ MSCtreated dogs. In the CNS, CSF infusion of TPP1-MSCs did not appear to delay or prevent neurologic disease signs; overall, there was no effect of treatment on lifespan, cognition, disease signs or brain atrophy. However, the two exceptions to this were in dogs that received lower passage MSCs. Dog 9 was the only dog to receive P3 TPP1-MSCs and demonstrated improved performance on the Tmaze task through 8 months of age, although was unable to perform the task at 10 months of age. Dog 6, who received P3 mCherry-MSCs, lived to 11.7 months of age, approximately one month longer than untreated dogs typically survive. Together, these results may indicate that lower passage number cells are in fact more effective.

Several other cell culture parameters were altered between the P5+ and P2 MSC-treated dogs, including reducing the amount of vector used to transduce cells and consistently using only $10 \%$ FBS to expand the cells. Media composition, particularly FBS percentage, may influence the immunosuppressive properties of cultured MSCs, so this should be more strictly controlled in the future (Khasawneh et al., 2019). In future studies, additional factors such as oxygen concentration and seeding density could also be considered (Choi et al., 2017; Grayson et al., 2007; Mareschi et al., 2012). Going forward, more 
consistent cell culture protocols may help reduce the variability in treatment efficacy and indicate which parameters are most critical. Also, by determining the secretion rate of the transduced MSCs at the time of harvest, the number of cells injected could be adjusted to achieve consistent amounts of hTPP1 secretion (at least in vitro).

An interesting possibility is that MSCs that do not produce TPP1 may confer some therapeutic benefit. This would not be surprising since untransduced MSCs have been used for their immunomodulatory properties and neuroinflammation occurs in the NCLs. Another major difference between dogs $1-5$ and dogs 6 - 9 in this study is that we used immunosuppressive medications with the first group but not the latter. They do not appear to be needed as latter group did not exhibit clinically evident treatment-related inflammation, and we thought it possible that they may in fact reduce the efficacy of the MSC treatment by interfering with their immunomodulatory properties. Data from dog 6 is encouraging but we have not been able to replicate these results. This dog received combined brain and eye treatment with low passage number cells, did not receive immunomodulatory medications, responded very well to treatment in the TPP1-MSC eye, appeared to have a treatment response in the GFP-MSC eye and lived approximately one month longer than is typical of untreated dogs despite receiving CSF infusion of only mCherry-MSCs. These data suggest that injection of MSCs may inhibit disease-related neuroinflammation. Assessment of inflammatory markers in the retina and CNS could indicate if this is indeed the case. 
The lab previously showed that intravitreal administration of TPP1-MSCs could partially prevent disease-related declines in retinal function (Tracy et al., 2016). In this study, we saw a similar benefit to the retina indicating that the previous findings are repeatable. Only partial preservation of retinal function has been achieved with this method to date, but modifications to the treatment could improve its efficacy and better understanding MSC behavior after injection will help direct these modifications. For example, if the cells do not survive throughout the entire study period, additional injections later in the disease course could be beneficial. Optimization of cell culture conditions could standardize the expansion and properties of the MSCs and produce more consistent results. Alternative methods of delivery such as implantation of encapsulated cells could also be considered. While the results are so far very promising, further optimization is required.

So far, we have not seen efficacy in the CNS. However, the slightly increased lifespan of dog 6 and improved T-maze performance of dog 9 may indicate some therapeutic benefit of the MSCs in those dogs. The fact that both dogs received lower passage MSCs and were not on any immunosuppressive medications indicates that future work should take these factors into consideration. Future studies could include optimized cell culture conditions, alternative delivery methods and more frequent injections over the disease course. A major hurdle to the development of MSC-based therapies is the lack of knowledge about cell survival and migration after administration, and studies in large animal models could help shed light on these important aspects of cell- 
based therapies. Encapsulation of the cells for CNS treatment would facilitate determination of cell survival and would allow for hTPP1 secretion into the CSF. Going forward, efforts should focus on determining if MSCs survive in the CNS, and if so for how long, as well as whether therapeutic concentrations of hTPP1 are obtainable with this method. MSC-based therapies such as this one will likely take considerable effort to implement but offer potential advantages over ERT and direct gene therapy approaches. 


\section{Intravitreal Gene Therapy Preserves Retinal Function in a}

\section{Canine Model of CLN2 Neuronal Ceroid Lipofuscinosis}

\subsection{Introduction}

In Chapter 2, we showed that periodic intravitreal injection of recombinant human TPP1 into the vitreous humor preserved retinal function and structure in dogs with CLN2 disease. Although this strategy has proven effective in slowing disease progression, the treatment will likely be quite expensive and will require relatively frequent, life-long intravitreal injections. This is fortunately a straightforward outpatient procedure and serious complications are rare, but repeated injections may entail complications such as endophthalmitis and adverse events have been reported with repeated intravitreal injections of antiVEGF agents used in treating retinal diseases (Falavarjani and Nguyen, 2013). We therefore sought to develop a gene therapy approach which could provide a long-term source of TPP1 with a single intravitreal injection as an alternative to the cell-mediated approach described in Chapter 3.

In this study, we tested the hypothesis that a single intravitreal injection of AAV2.hTPP1 provides a long-term source of enzymatically active TPP1 to retinal cells sufficient to preserve retinal function. Work in other models has shown that intravitreal administration of AAV2 vectors leads to successful transduction of cells in the inner retina, primarily retinal ganglion cells (Mowat et al., 2014; Yin et al., 2011). In the case of CLN2 disease, every cell need not be transduced to benefit from the treatment. When TPP1\% dogs received injections of AAV2.TPP1 
into the CSF, transduction was restricted almost exclusively to the ependymal cells lining the brain ventricles but the distribution of the TPP1 protein in the treated dogs was fairly widespread throughout the brain (Katz et al., 2015). Therefore, transduced retinal cells may produce enough TPP1 to meet their own needs and secrete excess TPP1 to supply non-transduced cells.

In the following study we tested whether a single intravitreal injection of AAV2.CAG.hTPP1 can provide therapeutic levels of TPP1 to the retina over an extended period in the canine model of CLN2 disease.

\subsection{Methods}

\subsubsection{Animals}

TPP1-/ purpose-bred miniature long-haired Dachshunds $(n=9)$ housed in a research colony at the University of Missouri - Columbia were used in this study. Breeding, genotyping, and husbandry were performed as described in 2.2.1. The study was approved by the University of Missouri Animal Care and Use Committee and was performed in accordance with the ARVO Statement for the Use of Animals in Ophthalmic and Vision Research. Ophthalmic exams were performed as described in 2.2.2. prior to enrollment in the study and dogs with any ophthalmic abnormalities were excluded.

\subsubsection{Treatments}

AAV2.CAG. $h$ TPP1 and AAV2.CAG.GFP stock vectors (SignaGen Laboratories, Rockville, MD) were combined with artificial CSF (1 mM sodium phosphate, $3 \mathrm{mM} \mathrm{KCl}, 1.4 \mathrm{mM} \mathrm{MgCl}_{2}, 0.8 \mathrm{mM} \mathrm{CaCl}_{2}$, and $148 \mathrm{mM} \mathrm{NaCl}, \mathrm{pH}$ 6.5) 
to achieve the appropriate titer in a volume of $100-250 \mu \mathrm{L}$ (dog D received 100 $\mu \mathrm{L}$ while all other dogs received 200-250 $\mu \mathrm{L}$ ). Both viral vectors employed AAV serotype 2 (AAV2) capsid and inverted terminal repeats with transgene expression under the control of the CAG promoter. The CAG promoter is a combination of the human cytomegalovirus immediate early enhancer element and the chicken $\beta$-actin promoter that results in ubiquitous overexpression of the transgene. The AAV2.CAG.GFP vector contained enhanced green fluorescent protein (GFP) cDNA and the AAV2.CAG. $h$ TPP1 vector contained 6x histidinetagged human TPP1 cDNA (Appendix B, Figure 4-1). Doses and ages at treatment are listed in Table 4-1. Intravitreal injection of the vector solution was performed as described in 3.2.4.1. All dogs received AAV2.CAG.GFP in the right eye and AAV2.CAG. $h T P P 1$ in the left eye. Dogs A, B, C, and D received escalating doses to determine the minimum effective dose while dogs E-I received a dose determined to have therapeutic benefit based on the results obtained from dogs $A, B, C$ and $D$. Dogs $B$ and $D$ also received intrathecal injections of AAVrh10.CAG.GFP and AAV9.CAG.hTPP1, respectively.

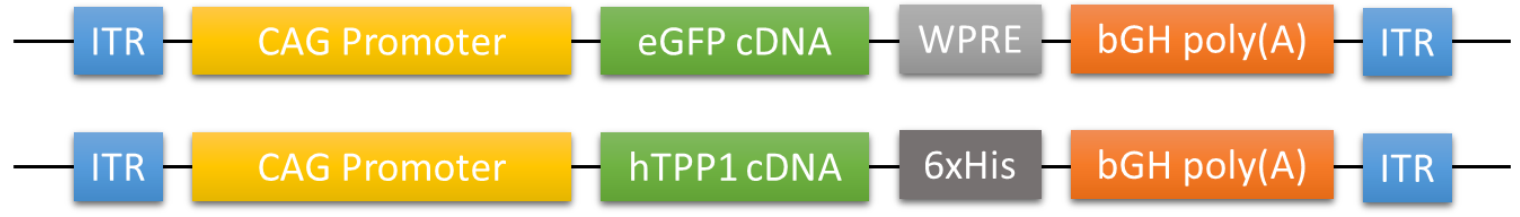

Figure 4-1: Diagram showing DNA constructs used in this study. ITR = AAV2 inverted terminal repeat; WPRE $=$ woodchuck hepatitis virus posttranscriptional regulatory element; bGH poly $(\mathrm{A})=$ bovine growth hormone polyadenylation signal; $6 \mathrm{xHis}=$ polyhistidine tag 


\section{Intravitreal Gene Therapy Treatments}

\begin{tabular}{ccc}
\hline Dog ID & $\begin{array}{c}\text { Age } \\
\text { (months) }\end{array}$ & $\begin{array}{c}\text { Dose } \\
\text { (vg/eye) }\end{array}$ \\
\hline Dog A & 4.9 & $6 \times 10^{9}$ \\
Dog B & 4.9 & $6 \times 10^{10}$ \\
Dog C & 4.8 & $6 \times 10^{11}$ \\
Dog D & 3.6 & $1 \times 10^{12}$ \\
Dog E & 3.8 & $1 \times 10^{12}$ \\
Dog F & 3.7 & $1 \times 10^{12}$ \\
Dog G & 4 & $1 \times 10^{12}$ \\
Dog H & 3.9 & $1 \times 10^{12}$ \\
Dog I & 3.9 & $1 \times 10^{12}$
\end{tabular}

Table 4-1: Intravitreal gene therapy treatments; $v g$ = viral genomes

To minimize treatment-related intraocular inflammation, a variety of antiinflammatory treatments were administered; these are summarized in Table 4-2. Two weeks prior to any vector administration, twice daily administration of $25 \mathrm{mg}$ cyclosporine and once daily administration of $2.5 \mathrm{mg} / \mathrm{kg}$ leflunomide was initiated. When the dog reached a weight of $4 \mathrm{~kg}$, the cyclosporine dose was increased to $35 \mathrm{mg}$. Cyclosporine and leflunomide were discontinued for $\operatorname{dog} \mathrm{A}$ 14 and 9 weeks after treatment, respectively. Dog B continued on cyclosporine throughout the entire study period but was discontinued from leflunomide 9 weeks post-treatment. Dog C was continuously maintained on cyclosporine but leflunomide was discontinued for 4 weeks after treatment. Dogs $E$ and F were discontinued from cyclosporine at 8 weeks post-treatment and from leflunomide at 5 weeks post-treatment; cyclosporine was resumed at 17 weeks posttreatment for $\operatorname{dog} E$ and 11 weeks post-treatment for dog $F$ when severe 
intraocular inflammation was noted. All other dogs $(D, G, H$, and I) remained on both cyclosporine and leflunomide for the entire study period.

Steroidal (Durezol or prednisolone acetate) and non-steroidal (Nevenac, ketorolac, or flurbiprofen) drops were administered to the corneal surface throughout the study to control inflammation as described in Table 4-2. Dog D was given three drops of prednisolone acetate $1 \%$ ophthalmic suspension OU and three drops of tropicamide $1 \%$ ophthalmic solution OU prior to vector injection. All other dogs were given one drop of Durezol OU four times daily and one drop of Nevenac OU three times daily starting 24 hours before the injection, and one drop of tropicamide OU prior to the injection. Dogs $B$ and $D$ received 1 $\mathrm{mg} / \mathrm{kg} /$ day oral prednisone for 3-4 days starting the day of intrathecal AAV administration followed by a $0.5 \mathrm{mg} / \mathrm{kg} /$ day taper for $3-4$ days. Prednisone was resumed in $\operatorname{dog} B$ following development of meningitis and was continued for 2 months. Durezol and Nevenac (or twice daily prednisolone acetate in the case of Dog D) were continued for at least one week following vector administration. Tropicamide or atropine drops were used as needed if inflammation occurred to dilate the pupil and prevent formation of synechiae. If no intraocular inflammation was appreciated, Durezol and Nevenac were replaced with prednisolone acetate and ketorolac. Durezol and Nevenac were resumed if at any point inflammation developed. All topical anti-inflammatories were discontinued in $\operatorname{dog}$ A 12 weeks after treatment. $2.2 \mathrm{mg} / \mathrm{kg}$ carprofen (Rimadyl) was administered twice daily as needed to address uncontrolled uveitis. 
Sub-Tenon's and intravitreal triamcinolone acetonide were administered at a dose of $2 \mathrm{mg}$. All dogs received sub-Tenon's triamcinolone in both eyes immediately following vector injection. Dog B received sub-Tenon's triamcinolone 5 and 10 weeks post-treatment to address ocular inflammation in the GFPcontrol eye. Dog C received sub-Tenon's triamcinolone 14 and 18 weeks posttreatment to address inflammation in the TPP1-treated eye. Dogs E and F received sub-Tenon's injections of $2 \mathrm{mg}$ triamcinolone acetonide every 2 weeks prophylactically. Dog E additionally received a single intravitreal injection of $2 \mathrm{mg}$ triamcinolone acetonide in the dorso-temporal region 20 weeks post-treatment to address active inflammation in the TPP1-treated eye. Dog G received subTenon's injections of $2 \mathrm{mg}$ triamcinolone 2 weeks after treatment and alternating sub-Tenon's and intravitreal injections of $2 \mathrm{mg}$ triamcinolone every 4 weeks thereafter. Dogs $\mathrm{H}$ and I initially alternated sub-Tenon's and intravitreal injections of triamcinolone like Dog $\mathrm{G}$ but, following the development of ocular inflammation 11-12 weeks after treatment, all monthly triamcinolone injections were intravitreal for the remainder of the study period (i.e., sub-Tenon's injections at 2 and 8 weeks only). 


\begin{tabular}{|c|c|c|c|c|c|c|}
\hline & $\begin{array}{l}\text { Dose } \\
\text { (vg) }\end{array}$ & Cyclosporine* & Leflunomide* & Topical Drugs & Triamcinolone** & $\begin{array}{l}\text { Inflammation } \\
\text { in TPP1 eye }\end{array}$ \\
\hline $\operatorname{Dog} A$ & $6 \times 10^{9}$ & $d / c 14 w$ & $d / c 9 w$ & $\begin{array}{l}\text { 0-5w: Durezol \& Nevenac } \\
\text { 5-7w: ketorolac \& prednisolone }\end{array}$ & & \\
\hline Dog B & $6 \times 10^{10}$ & $\begin{array}{l}\text { entire study } \\
\text { period }\end{array}$ & $d / c 9 w$ & $\begin{array}{l}\text { 0-9w: Durezol \& Nevenac } \\
\text { 9-11w: ketorolac \& prednisolone } \\
\text { 11w+: ketorolac }\end{array}$ & $\begin{array}{l}\text { Sub-tenon's at } 5 \& \\
10 w\end{array}$ & \\
\hline Dog C & $6 \times 10^{11}$ & $\begin{array}{l}\text { entire study } \\
\text { period }\end{array}$ & $d / c 4 w$ & $\begin{array}{l}\text { 0-4w: Durezol \& Nevenac } \\
\text { 4-5w: ketorolac \& prednisolone } \\
\text { 5-17w: Durezol \& Nevenac }+/- \text { atropine, } \\
\text { carprofen } \\
\text { 17w+: ketorolac \& prednisolone }\end{array}$ & $\begin{array}{l}\text { Sub-tenon's at } 15 \& \\
18 \mathrm{w}\end{array}$ & $4.5 w$ \\
\hline Dog D & $1 \times 10^{12}$ & $\begin{array}{l}\text { entire study } \\
\text { period }\end{array}$ & $\begin{array}{l}\text { entire study } \\
\text { period }\end{array}$ & $\begin{array}{l}\text { 0-1w: prednisolone } \\
\text { 3-8w: Durezol \& Nevenac, tropicamide } \\
\text { 11w+: prednisolone, flurbiprofen }+/- \\
\text { carprofen }\end{array}$ & & $3 w$ \\
\hline $\operatorname{Dog} E$ & $1 \times 10^{12}$ & $\begin{array}{l}\mathrm{d} / \mathrm{c} 8 \mathrm{w} ; \\
\text { resume } 17 \mathrm{w}\end{array}$ & $d / c 5 w$ & $\begin{array}{l}\text { 0-4w: Durezol \& Nevenac } \\
\text { 4-16w: ketorolac \& prednisolone } \\
\text { 17-20w: Durezol, Nevenac, atropine, } \\
\text { tropicamide, carprofen } \\
\text { 20w+: ketorolac \& prednisolone +/- } \\
\text { carprofen, tropicamide, atropine }\end{array}$ & $\begin{array}{l}\text { Sub-tenon's every } \\
2 \mathrm{w} \\
\text { Intravitreal at } 20 \mathrm{w}\end{array}$ & $17 w$ \\
\hline Dog F & $1 \times 10^{12}$ & $\begin{array}{l}\mathrm{d} / \mathrm{c} 8 \mathrm{w}, \\
\text { resume } 11 \mathrm{w}\end{array}$ & $d / c 5 w$ & $\begin{array}{l}\text { 0-4w: Durezol \& Nevenac } \\
\text { 4-11w: ketorolac \& prednisolone } \\
\text { 11w+: Durezol, Nevenac, atropine, } \\
\text { tropicamide +/- carprofen }\end{array}$ & $\begin{array}{l}\text { Sub-tenon's every } \\
2 \mathrm{w}\end{array}$ & $11 w$ \\
\hline Dog G & $1 \times 10^{12}$ & $\begin{array}{l}\text { entire study } \\
\text { period }\end{array}$ & $\begin{array}{l}\text { entire study } \\
\text { period }\end{array}$ & $\begin{array}{l}\text { 0-1w: Durezol \& Nevenac } \\
\text { 1-17w: ketorolac \& prednisolone } \\
\text { 17w+: ketorolac \& prednisolone }+/- \\
\text { tropiacmide, atropine }\end{array}$ & $\begin{array}{l}\text { Sub-tenon's: } 2,4,6 \text {, } \\
\text { 12, 14, 18, 20, 24w } \\
\text { Intravitreal: } 8,16 \mathrm{w}\end{array}$ & $25 w$ \\
\hline Dog H & $1 \times 10^{12}$ & $\begin{array}{l}\text { entire study } \\
\text { period }\end{array}$ & $\begin{array}{l}\text { entire study } \\
\text { period }\end{array}$ & $\begin{array}{l}\text { 0-1w: Durezol \& Nevenac } \\
\text { 1-11w: ketorolac \& prednisolone } \\
\text { 11-16w: ketorolac \& prednisolone +/- } \\
\text { tropicamide \& carprofen } \\
\text { 19w+: ketorolac }\end{array}$ & $\begin{array}{l}\text { Sub-tenon's: } 2,8 w \\
\text { Intravitreal: } 4,11 \text {, } \\
16,20,24 w\end{array}$ & $10-11 w$ \\
\hline Dog I & $1 \times 10^{12}$ & $\begin{array}{l}\text { entire study } \\
\text { period }\end{array}$ & $\begin{array}{l}\text { entire study } \\
\text { period }\end{array}$ & $\begin{array}{l}\text { 0-1w: Durezol, Nevenac, atropine } \\
\text { 1-2w: ketorolac, prednisolone, atropine } \\
\text { 2-12w: ketorolac \& prednisolone } \\
\text { 12-14w: Nevenac, prednisolone, } \\
\text { carporfen } \\
\text { 14-25w: ketorolac }+ \text { /- carprofen }\end{array}$ & $\begin{array}{l}\text { Sub-tenon's: } 2,8 w \\
\text { Intravitreal: } 4,12 \text {, } \\
16,20,24 w\end{array}$ & $11-12 w$ \\
\hline
\end{tabular}

Table 4-2: Summary of anti-inflammatory treatments. $\mathrm{w}=$ weeks; $\mathrm{d} / \mathrm{c}=$ discontinued ${ }^{*}$ Cyclosporine and leflunomide were initiated 2 weeks before treatment. ${ }^{* *}$ All dogs received sub-Tenon's triamcinolone immediately following vector injection. 


\subsubsection{In vivo Measures to Assess Retinal Function and Structure}

Retinal function was assessed with bilateral ERG recordings, performed monthly following a baseline measurement taken immediately prior to treatment, as described in 2.2 .5 . Midazolam $(0.25 \mathrm{mg} / \mathrm{kg})$ was used as needed for additional sedation during ERG recording. SLO and OCT imaging were performed as described in 2.2.6 prior to vector administration and then monthly to document inflammation, CMR lesions and GFP fluorescence.

\subsubsection{Euthanasia and Necropsy}

Dogs were euthanized at end-stage neurological disease as described in 2.2.7 following final ERG and retinal imaging procedures. Eyes were collected from all dogs immediately following euthanasia and fixed in immuno fixative for further processing and histology. Retinas from dogs $A-D$ and $\operatorname{dog} G$ were dissected as described in section 2.2.8 (Figure 2-1).

Region A2 from the central superior area of dog G's GFP-control eye was examined with a fluorescence stereoscope and images of the region were captured before embedding for cryostat sectioning and fluorescence microscopy. The A2 region of the eyecup was dissected out and incubated in $0.17 \mathrm{M}$ sodium cacodylate buffer at room temperature for at least $30 \mathrm{~min}$. The sample was then incubated at room temperature in $10 \mathrm{~g}$ sucrose per $100 \mathrm{~mL} 170 \mathrm{mM}$ cacodylate buffer ( $\mathrm{pH}$ 7.4) for one hour, $20 \mathrm{~g}$ sucrose per $100 \mathrm{~mL}$ cacodylate buffer for two hours, a 1:1 mixture of $20 \mathrm{~g}$ sucrose per $100 \mathrm{~mL}$ cacodylate buffer and 
TissueTek O.C.T. Compound (Sakura FineTek USA, Inc., Torrance, CA) for 1.5 hours. The sample was incubated in TissueTek O.C.T. Compound in a plastic mold on wet ice for 45 minutes then frozen in the mold on dry ice. $8 \mu \mathrm{m}$ cryostat sections were cut with a Microm HM525 cryostat (Thermo Fisher Scientific, Waltham, MA) and thaw-mounted on SuperFrost Plus slides (Thermo Fisher Scientific, Waltham, MA), then examined with a fluorescence microscope.

Region B2 from the superior mid-peripheral retina of Dog $G$ was paraffinembedded and immunolabeled with an anti-rhTPP1 antibody (clone 2E12, catalogue \# 852202, BioLegend, San Diego, CA) at a concentration of $1: 500$ by the Veterinary Medicine Diagnostics Laboratory at the University of Missouri Columbia. Sections were deparaffinized and hydrated to water with xylene and ethanol. Heat induced epitope retrieval was done with Diva Decloaker (Biocare Medical, Pacheco, CA) in a decloaking chamber. Sections were blocked with $3 \%$ hydrogen peroxide then Background Sniper (Biocare Medical) and incubated with the anti-rhTPP1 antibody (1 hour), secondary antibody solution (30 minutes, undiluted anti-mouse MACH 2; Biocare Medical), chromagen (Romulin Red; Biocare Medical) and counterstain (CAT hematoxylin; Biocare Medical). Sections were dehydrated and cleared with ethanol and xylene.

\subsubsection{Statistical Analysis}

All statistical tests were performed using SigmaPlot (Systat Software Inc., San Jose, CA). Data were subjected to the Shapiro-Wilk test to confirm normal distribution. Repeated measures 2-way ANOVA was used to compare ERG data from CLN2-affected eyes treated with AAV2.hTPP1 to those eyes treated with 
AAV2.GFP to determine if the treatment was able to prevent deficits in ERG bwave amplitudes related to CLN2 disease progression. Follow-up pairwise comparisons were performed with the Holm-Sidak correction $(\alpha=0.05)$ to control family-wise error rate. Because of missing datapoints due to intraocular inflammation, data from treated dogs were grouped into four age groups: 4 months (3-4.9), 6 months (5-6.9), 8 months (7-8.9) and 10 months (9-10.9) of age. The average amplitude was used when a dog had more than one recording in an age range. Recordings taken during active inflammation were omitted if at least one response was nonrecordable during that session but recovered the following month. Recordings taken from dog E when the TPP1-treated fundus could not be visualized due to inflammation were omitted.

\subsection{Results}

\subsubsection{Transgene Expression}

A dose escalation series was performed to determine, approximately, the minimum effective dose. Dogs A, B and C received doses of $10^{9}, 10^{10}$ and $10^{11}$ vg/eye, respectively (see Table 4-1). Fluorescence SLO fundus imaging of the GFP-control eyes indicated that all three doses resulted in successful, dosedependent transduction of the retina (Figure 4-2); however, none of these doses improved ERG amplitudes in the treated eyes (data not shown). 

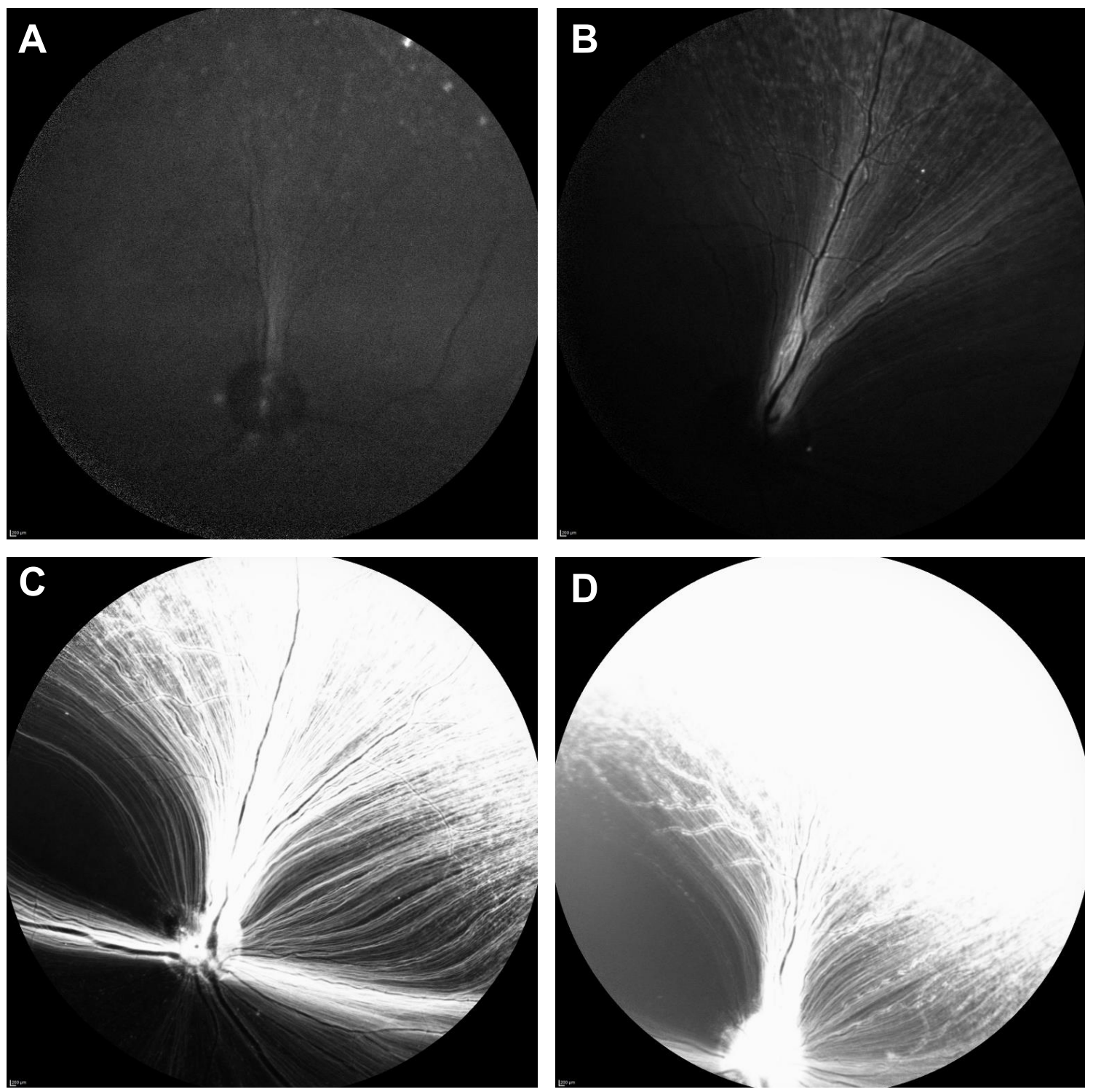

Figure 4-2: Fluorescence SLO images of dogs $A(A), B(B), C$ (C) and $G(D)$ showing dose-dependent transduction of the retina with AAV2.CAG.GFP. Doses of $6 \times 10^{9}(\mathrm{~A})$ and $6 \times 10^{10} \mathrm{vg}(\mathrm{B})$ resulted in only modest GFP expression. $6 \times 10^{11}$ $\mathrm{vg}(\mathrm{C})$ resulted in widespread GFP expression but did not preserve ERG amplitudes. Only the highest dose tested, $1 \times 10^{12} \mathrm{vg}$, both resulted in widespread GFP expression (D) and preserved retinal function.

Dogs E-I were treated with a therapeutic dose of $10^{12} \mathrm{vg} / \mathrm{eye}$. Fluorescence indicating successful transgene expression in the GFP-control eye was apparent as early as 2 weeks after administration with fluorescence SLO imaging (Figure 
4-2). This modality is not quantitative, but the fluorescence signal appeared to increase through 8-12 weeks post-treatment (Figure 4-3). Fluorescence was especially prominent along the major retinal vessels. This is consistent with previous reports and thought to be due to a less compact inner limiting membrane - the basement membrane made up of Müller cell end feet that defines the border between the retina and the vitreous and is a major barrier to AAV transduction of the retina via intravitreal injection - along retinal blood vessels (Dalkara et al., 2009; Pavlou et al., 2021). An unusual raised vessel pattern was noted in Dog G prior to treatment, and the dog subsequently developed a vitreal hemorrhage at this site within the two weeks following treatment. This was thought to be unrelated to the therapy shortly after treatment and resulted in especially strong GFP fluorescence in this area even after the hemorrhage resolved. Likely due to the trajectory of the needle during injection, the superior retina, especially the superior nasal quadrant, was the primary site of transduction. Dog C's dose was split and injected both nasally and temporally; however, as the higher therapeutic dose resulted in widespread GFP expression across much of the superior retina with a single injection site, this was not repeated. The fluorescence signal plateaued at approximately 8 to 12 weeks post-injection and persisted without apparent decrease through the remainder of the study period for each dog. The pattern of transduction indicates that the retinal ganglion cells, whose axons project to the optic nerve head, are strongly transduced by the vector. 

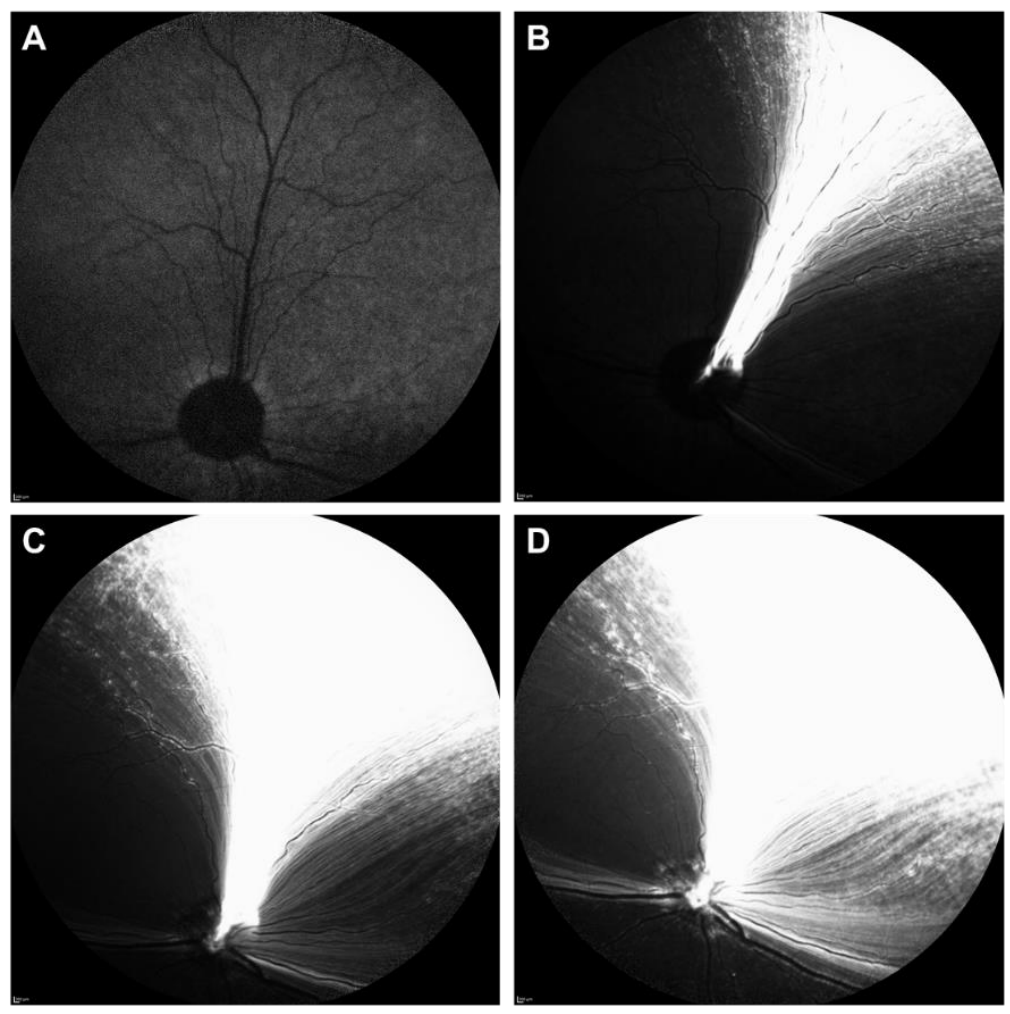

Figure 4-3: Fluorescence SLO images of the GFP-control retina of dog $\mathrm{E}$ at 0 (A), 2 (B), 4 (C) and 8 (D) weeks post-treatment showing increasing fluorescent signal over the first 8 weeks after treatment.

The retinas from most of the treated dogs have not yet been processed for histological evaluation and immunohistochemistry. However, preliminary data from dog $\mathrm{G}$ who was treated with $10^{12} \mathrm{vg}$ indicates that at this dose the GFP (Figure 4-4) and TPP1 (Figure 4-5) proteins continued to be expressed at the end of the study period. In both cases, labeling was most pronounced in the inner retina. However, scattered RPE cells were also heavily labeled. Photoreceptor cells did not appear to be transduced. Additionally, while a thorough assessment remains to be done, there appears to be preservation of retinal structure in the TPP1-treated eye of dog G relative to the GFP-control eye (Figure 4-5). The 
GFP-control retina is considerably thinner and appears to contain fewer nuclei in the inner and outer nuclear layers.

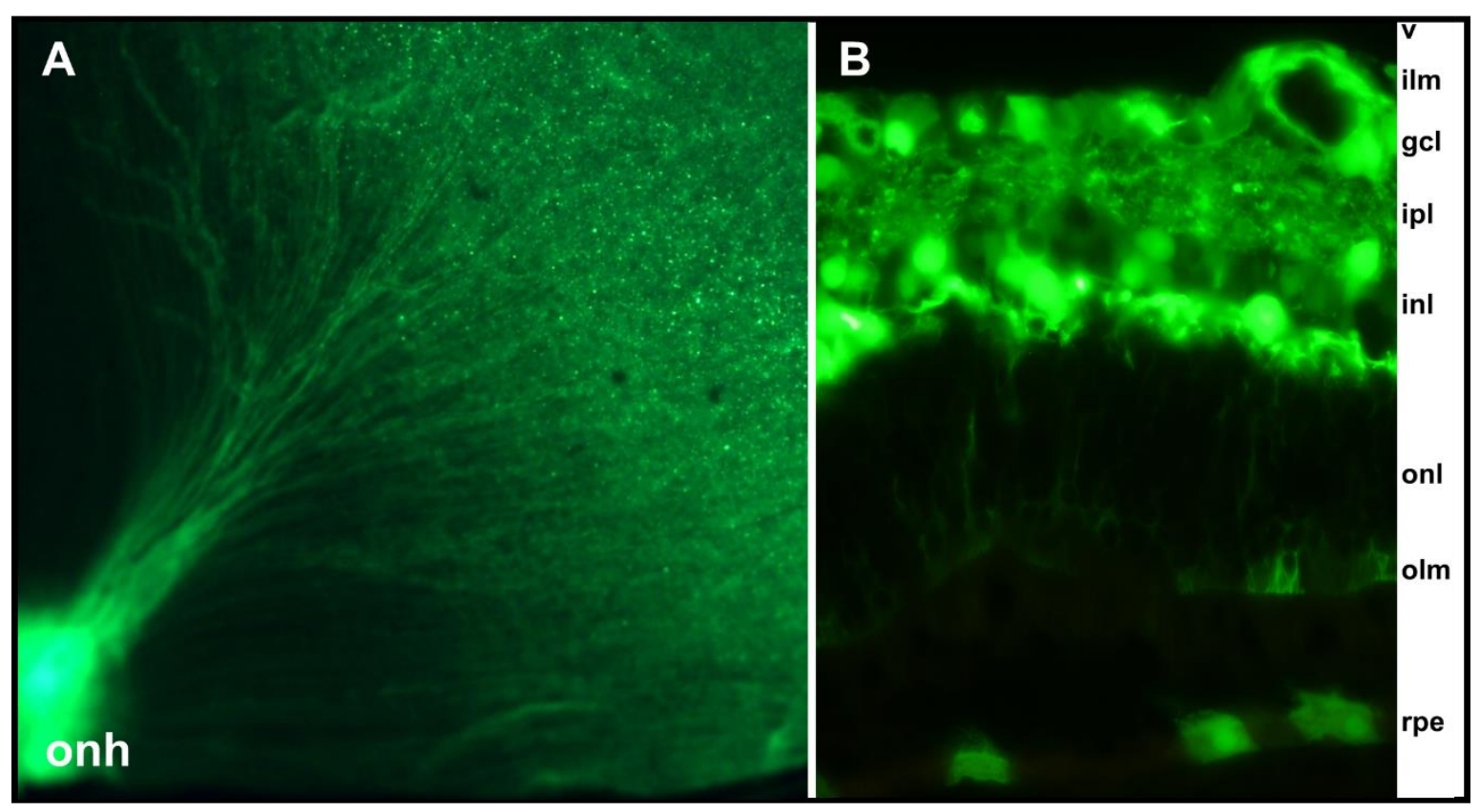

Figure 4-4: Low magnification fluorescence stereoscope image of the central superior retina from the GFP-control retina of Dog $G(A)$ and a fluorescence micrograph of a section from the same region $(B)$. onh $=$ optic nerve head; $v=$ vitreous; ilm = inner limiting membrane; gcl = ganglion cell layer; ipl = inner plexiform layer; inl = inner nuclear layer; onl = outer nuclear layer; olm = outer limiting membrane; rpe = retinal pigment epithelium 

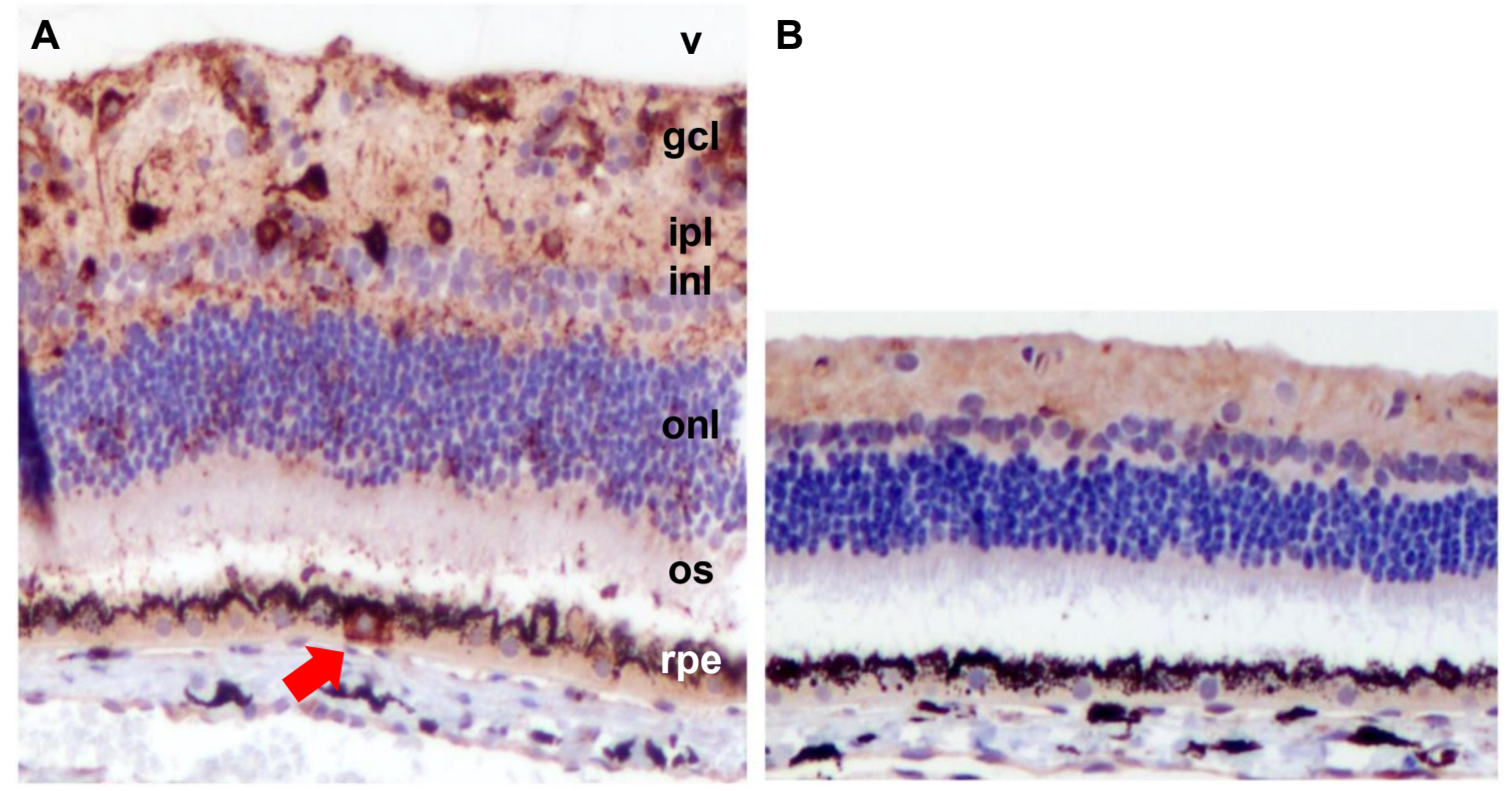

Figure 4-5: Micrographs of TPP1-stained retinas from the superior midperipheral region of TPP1-treated (A) and GFP-control (B) eyes of dog G. The arrow shows a labeled RPE cell. $\mathrm{v}=$ vitreous; $\mathrm{gcl}=$ ganglion cell layer; $\mathrm{ipl}=$ inner plexiform layer; inl = inner nuclear layer; onl = outer nuclear layer; os = photoreceptor outer segments; rpe = retinal pigment epithelium

\subsubsection{Ocular Inflammation}

Ocular inflammation was observed in only one GFP-control eye. Dog B received an intrathecal injection of AAVrh10.CAG. GFP in addition to the intravitreal injections. This dog developed meningitis with mononuclear pleocytosis in the CSF 2 weeks after intrathecal injection and anterior uveitis in the GFP-control eye. Interestingly, dog B was the only dog to develop inflammation in the GFP eye and did not develop inflammation in the TPP1treated eye. This suggests that exposure of large amounts of GFP to the immune system resulted in an immune response against GFP, which is intracellular and generally thought to be non-immunogenic. 
Doses of $10^{9}$ and $10^{10} \mathrm{vg}$ did not result in inflammation in the TPP1treated eyes. In fact, all immunosuppressive and anti-inflammatory drugs were discontinued in $\operatorname{dog} \mathrm{A}\left(10^{9} \mathrm{vg}\right)$ by 9 weeks post-treatment, and the dog was on no such medications for the remainder of the study period. Dog C, treated with $10^{11} \mathrm{vg}$, exhibited relatively mild anterior and posterior uveitis, papilledema and posterior synechia beginning 4.5 weeks after treatment.

All dogs treated with $10^{12} \mathrm{vg}$ exhibited intraocular inflammation in the TPP1-treated eye (Figure 4-6). The fact that inflammation was consistently noted in only TPP1-treated eyes suggests that the symptoms are a response to hTPP1 production rather than the AAV2 vector. Dog D developed inflammation characterized by anterior and posterior uveitis, low IOP, papilledema and posterior synechia in the TPP1-treated eye 3 weeks after treatment. This dog was treated with systemic immunosuppressants and topical anti-inflammatories but did not receive triamcinolone except for immediately following treatment. Inflammation gradually improved; at the end of the study period, only corneal dystrophy, mild papilledema and synechia remained. Dogs $E$ and $F$ received biweekly sub-Tenon's injections of triamcinolone which appeared to delay the onset of inflammation to 17 and 11 weeks, respectively, when both dogs developed severe intraocular inflammation. In contrast to dog D, cyclosporine and leflunomide were discontinued for dogs $E$ and $F 5$ to 8 weeks after treatment, though cyclosporine was resumed for both once inflammation developed. Dog F exhibited anterior and posterior uveitis, low IOP, papilledema and posterior synechia at 11 weeks post-treatment in the TPP1-treated eye. 
While the uveitis resolved, synechia caused permanent miosis. Dog E developed severe anterior and posterior uveitis, papilledema and mydriasis due to posterior synechia 17 weeks after treatment. At 20 weeks post-treatment, the fundus could not be visualized due to vitreal opacity. Given the primarily posterior location of the inflammation, we considered that intravitreal triamcinolone may result in better distribution to the site of inflammation than topical anti-inflammatories or sub-Tenon's injection. Intravitreal triamcinolone was administered to both eyes and uveitis was controlled despite persistent mild swelling of the optic nerve head. Given the success of intravitreal triamcinolone at controlling severe uveitis in this dog, we decided to test prophylactic intravitreal triamcinolone in dog G. As the half-life of triamcinolone in the vitreous is around one month, we alternated sub-Tenon's and intravitreal triamcinolone injections to avoid excessive buildup of the drug in the vitreous (Beer et al., 2003; Kim et al., 2006). Moreover, because intravitreally injected triamcinolone can persist as a clinically visible depot for several months, we were concerned that this might impede ERG assessment (Molleda et al., 2008; Vedantham et al., 2005). Dog G and subsequent dogs $\mathrm{H}$ and I were additionally maintained on cyclosporine and leflunomide for the entire study period. At 17 weeks post-treatment, dog G exhibited sub-clinical inflammation characterized by mydriasis due to posterior synechia and low IOP. Mild anterior uveitis developed 25 weeks after treatment. We considered this quite successful compared to our past experience, and intended to repeat this protocol in two subsequent dogs, $\mathrm{H}$ and $\mathrm{I}$. However, both dogs developed severe inflammation characterized by uveitis and posterior 
synechia approximately 10-12 weeks after treatment. Dog $\mathrm{H}$ also exhibited a small retinal hemorrhage and dog I papilledema. At this point, sub-Tenon's triamcinolone was discontinued, and intravitreal triamcinolone was administered monthly, which seemed to be well tolerated. Intravitreal triamcinolone was administered per protocol to dog I and one week early to dog $\mathrm{H}$ when inflammation was noted. Uveitis was controlled with treatment, although the final intravitreal injection of triamcinolone caused uveitis in the TPP1-treated eye of dog $\mathrm{H}$.

We found that intravitreal injection of triamcinolone into an eye with active inflammation appears to result in a much more diffuse distribution pattern compared to injection into a healthy eye, potentially due to degeneration of the vitreous. In the GFP-control eyes, the drug persisted as a small depot that was typically located in the periphery and frequently dissipated within a month, whereas in inflamed eyes the drug tended to disperse much more, resulting in a large central mass that was often still visible a month later. In Figure 4-6, this can be seen in $\operatorname{dog} E$. 


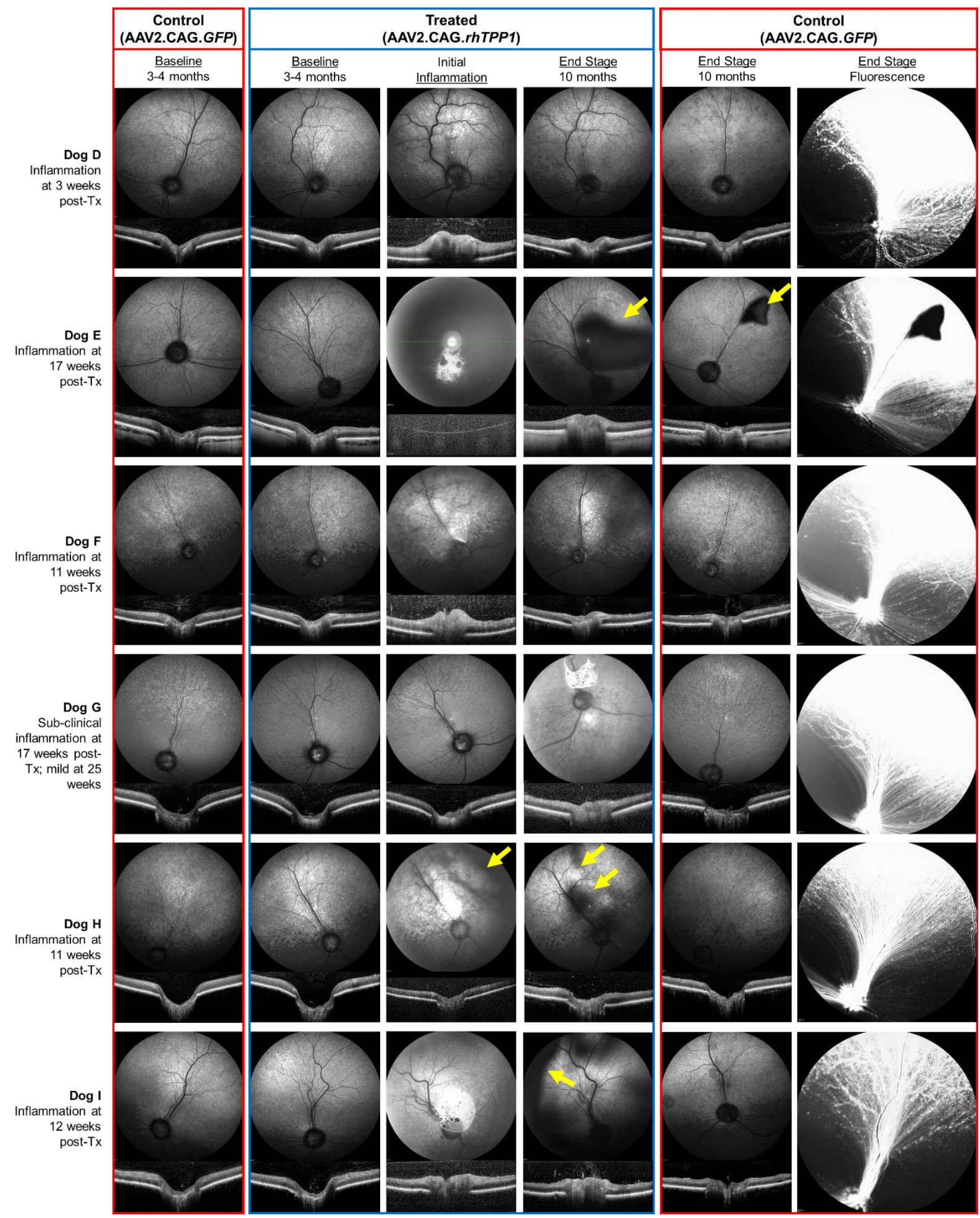

Figure 4-6: SLO fundus images and corresponding OCT images from the center of the optic nerve head from dogs treated with the therapeutic dose of AAV2.CAG.hTPP1. Fluorescence SLO images from the GFP-control eye are shown at the end of each row. Yellow arrows indicate deposits of intravitreal triamcinolone. 
While the immunosuppressive protocol for $\operatorname{dog} \mathrm{G}$ and $\operatorname{dogs} \mathrm{H}$ and I was intended to be identical, in actuality dog $\mathrm{G}$ received a different cyclosporine formulation. With the exception of $\operatorname{dog} \mathrm{G}$, all dogs in this study received the Sandimmune vegetable oil-based oral preparation. Dog $G$ was started on the Sandimmune preparation but switched to the modified Neoral ultramicronized formulation 10 weeks after treatment and this was continued through the remainder of the study period. The Neoral formulation has increased bioavailability compared to the Sandimmune preparation resulting in higher and more consistent absorption (Colombo and Egan, 2010). While we routinely assess blood levels of cyclosporine in study dogs, blood concentrations between and even within patients are known to vary markedly, making comparison to established reference ranges difficult (Archer et al., 2014, 2011). We therefore submitted samples from dogs $\mathrm{H}$ and I for a cyclosporine pharmacodynamic assay which assesses T cell function via interleukin-2 qRT-PCR (College of Veterinary Medicine, Mississippi State University, MS). This assay requires fresh whole blood, so we were unable to retrospectively assess dog G's T cell suppression. Dog $\mathrm{H}$ exhibited only moderate suppression of T cells while dog I exhibited low T cell suppression even though both dogs were maintained on leflunomide and cyclosporine, which both suppress T cell activity, throughout the study period. 


\subsubsection{Retinal Function}

Dog D developed severe ocular inflammation 3 weeks after treatment.

ERG recordings performed 6 and 10 weeks after treatment ( 5 and 6 months of age) did not show any preservation of retinal function in the treated eye (Figure 4-7). One additional ERG recording was performed 23 weeks after treatment (8.8 months of age). Ocular inflammation had largely resolved in the intervening time, and while ERG b-wave amplitudes were reduced from normal in both eyes, there was partial preservation of rod, cone and mixed rod and cone b-wave amplitudes in the treated eye relative to the control eye.
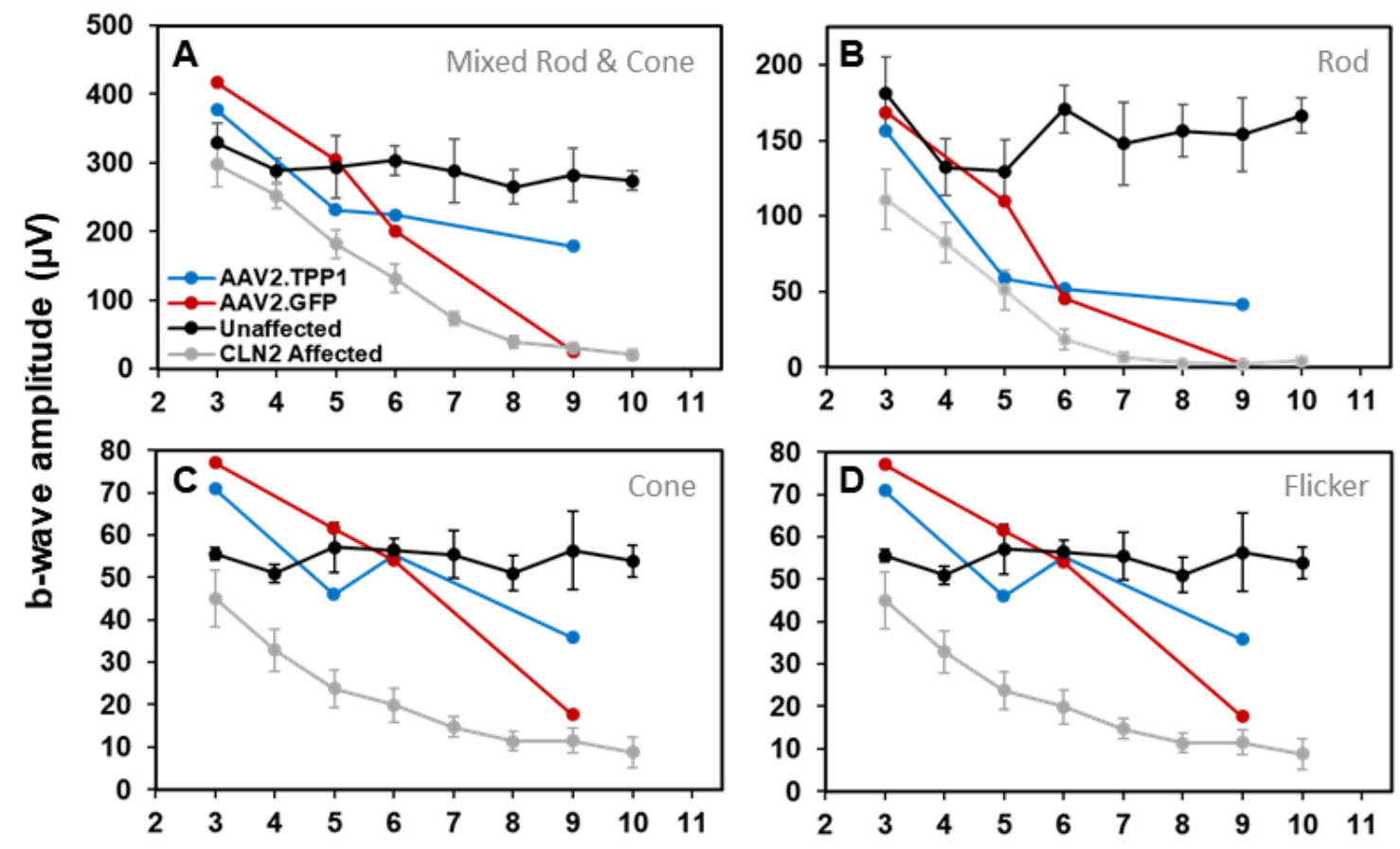

age (months)

Figure 4-7: Mixed rod and cone (A), pure rod (B), pure cone $(C)$ and cone flicker (D) response amplitudes from dog $D$. Black and gray traces represent historical data \pm SEM from normal $(n=7)$ and CLN2-affected $(n=6)$ untreated dogs, respectively, from the same research colony. 
Dogs $\mathrm{E}$ and $\mathrm{F}$ were then treated simultaneously. To better control inflammation, both dogs received bi-weekly sub-Tenon's injections of triamcinolone. Prior to onset of inflammation, dog E's mixed rod and cone and pure rod b-wave amplitudes remained normal in the TPP1-treated eye (Figure 48). Severe ocular inflammation developed at 8.5 months of age, and all b-wave amplitudes in the TPP1-treated eye at 9 months, while inflammation was present, were reduced from those recorded at 7.5 months prior to onset of inflammation. A single intravitreal injection of triamcinolone was administered at 9 months of age. Once inflammation began to resolve, amplitudes rebounded and at the end of the study period all b-wave responses were normal or above normal. ERG waveforms from this dog are shown in Figure 4-9. For dog F, mixed rod and cone and pure rod response amplitudes were initially preserved at normal levels through 5.5 months of age while cone and cone flicker response amplitudes declined (Figure 4-10). Severe inflammation occurred at 6.5 months of age and ERG amplitudes were dramatically reduced in the TPP1-treated eye at 6.5 months of age while the amplitudes in the GFP-control eye continued to decline steadily. As seen with dogs D and E, ERG amplitudes rebounded as inflammation resolved; however, dog $\mathrm{F}$ became permanently miotic due to synechia which prevents pupil dilation and limits the amount of light reaching the retina during ERG flash administration. This effectively makes the actual flash intensity indeterminable for this dog and the results are difficult to interpret. Despite this, the mixed rod and cone response returned to near baseline and 
pure rod and pure cone amplitudes were partially preserved in the TPP1-treated eye.

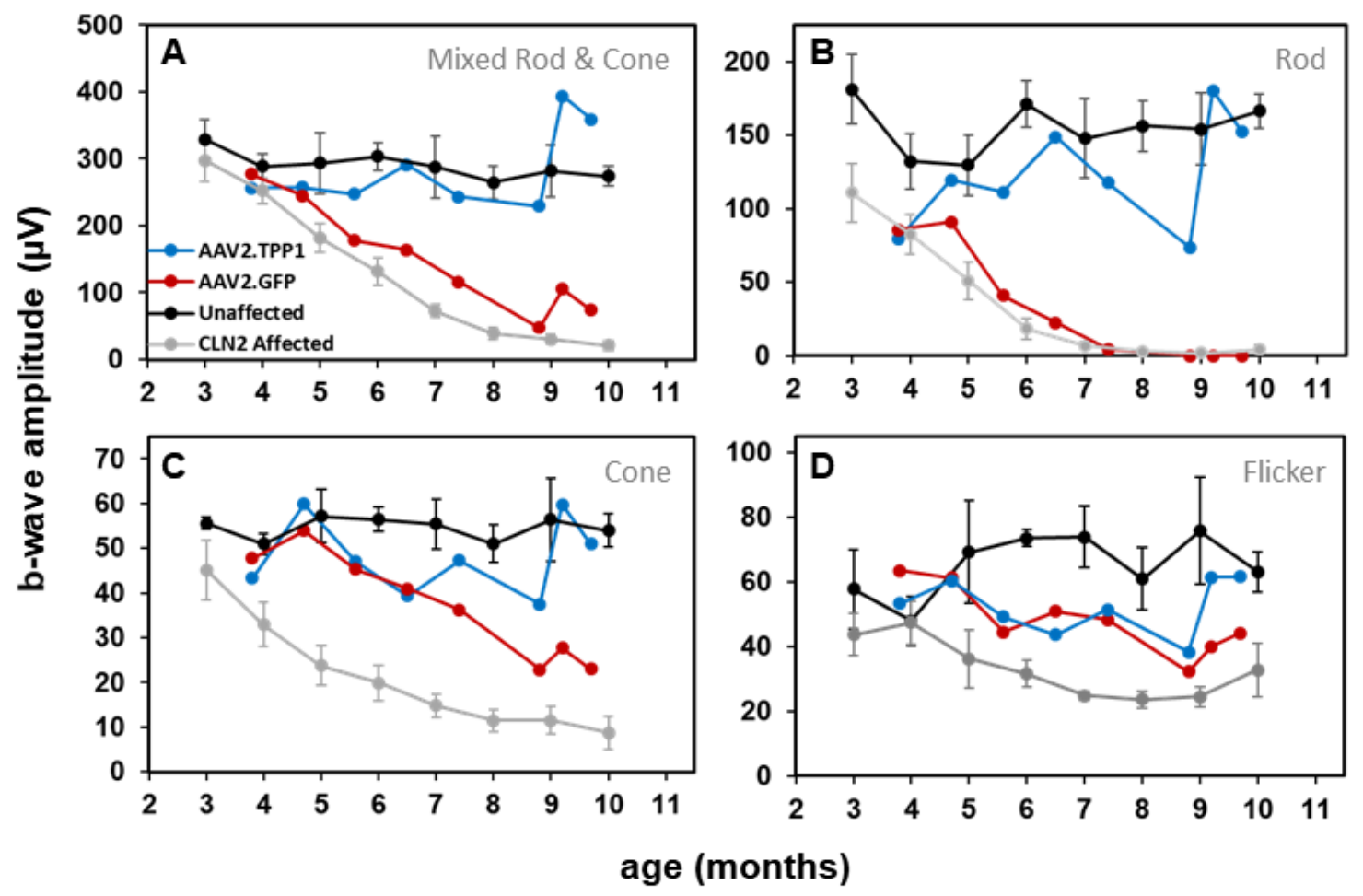

Figure 4-8: Mixed rod and cone (A), pure rod (B), pure cone (C) and cone flicker (D) response amplitudes from dog $\mathrm{E}$. Black and gray traces represent historical data \pm SEM from normal $(n=7)$ and CLN2-affected $(n=6)$ untreated dogs, respectively, from the same research colony. 


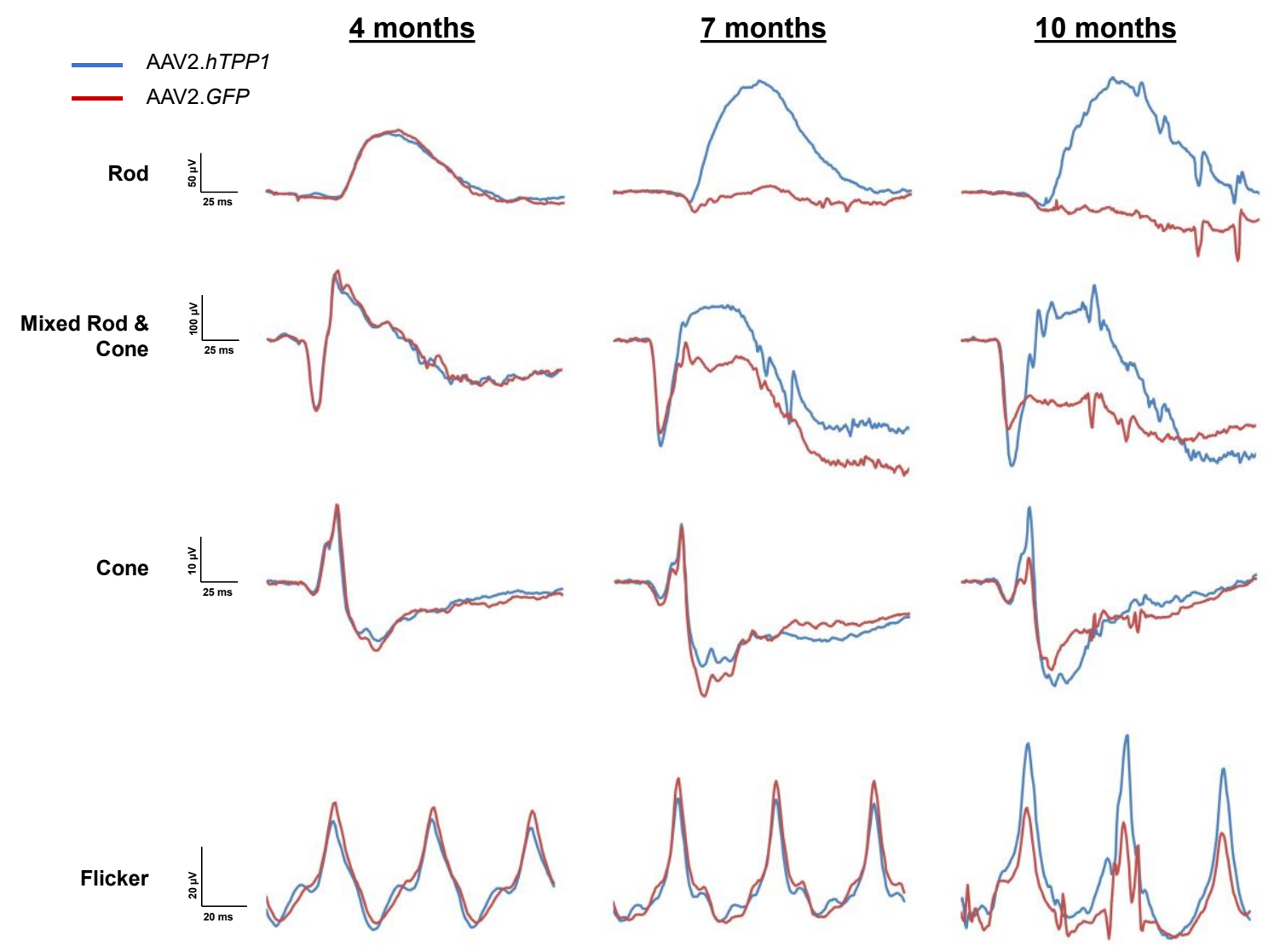

Figure 4-9: ERG waveforms from dog $E$ at 4, 7 and 10 months of age.

Responses from the AAV2.CAG.hTPP1 treated eye (blue traces) remained normal or greater than normal throughout the study period, while the responses from the AAV2.CAG.GFP control eye (red traces) declined as expected with age. 


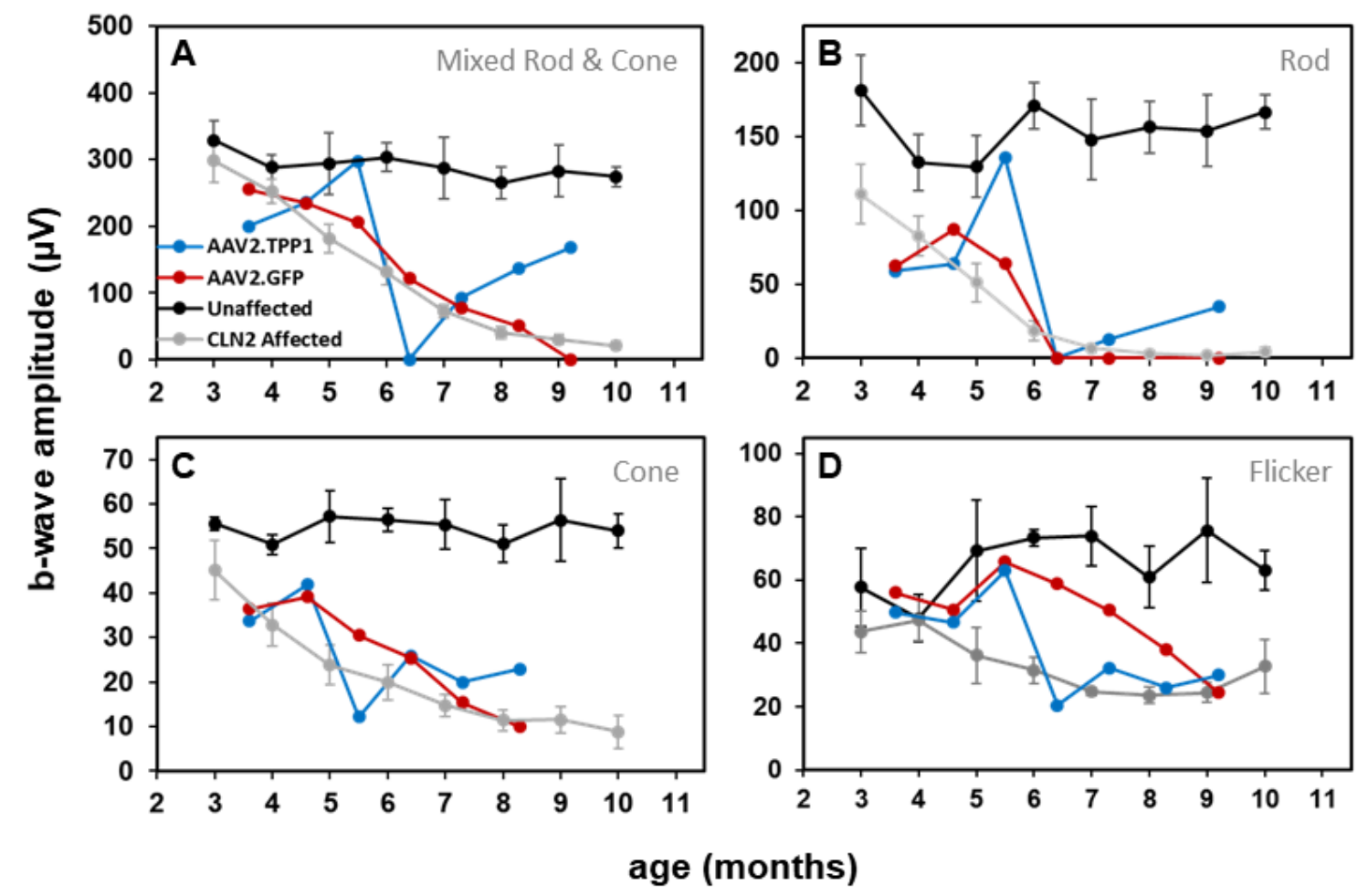

Figure 4-10: Mixed rod and cone (A), pure rod (B), pure cone $(C)$ and cone flicker (D) response amplitudes from dog F. Black and gray traces represent historical data \pm SEM from normal $(n=7)$ and CLN2-affected $(n=6)$ untreated dogs, respectively, from the same research colony.

Dog G's immunosuppression protocol included modified cyclosporine and alternating monthly sub-Tenon's and intravitreal triamcinolone injections. Mixed rod and cone b-wave amplitudes remained largely normal through the study period (Figure 4-11). Pure rod, pure cone and cone flicker responses declined over time but were partially preserved in the treated eye. 


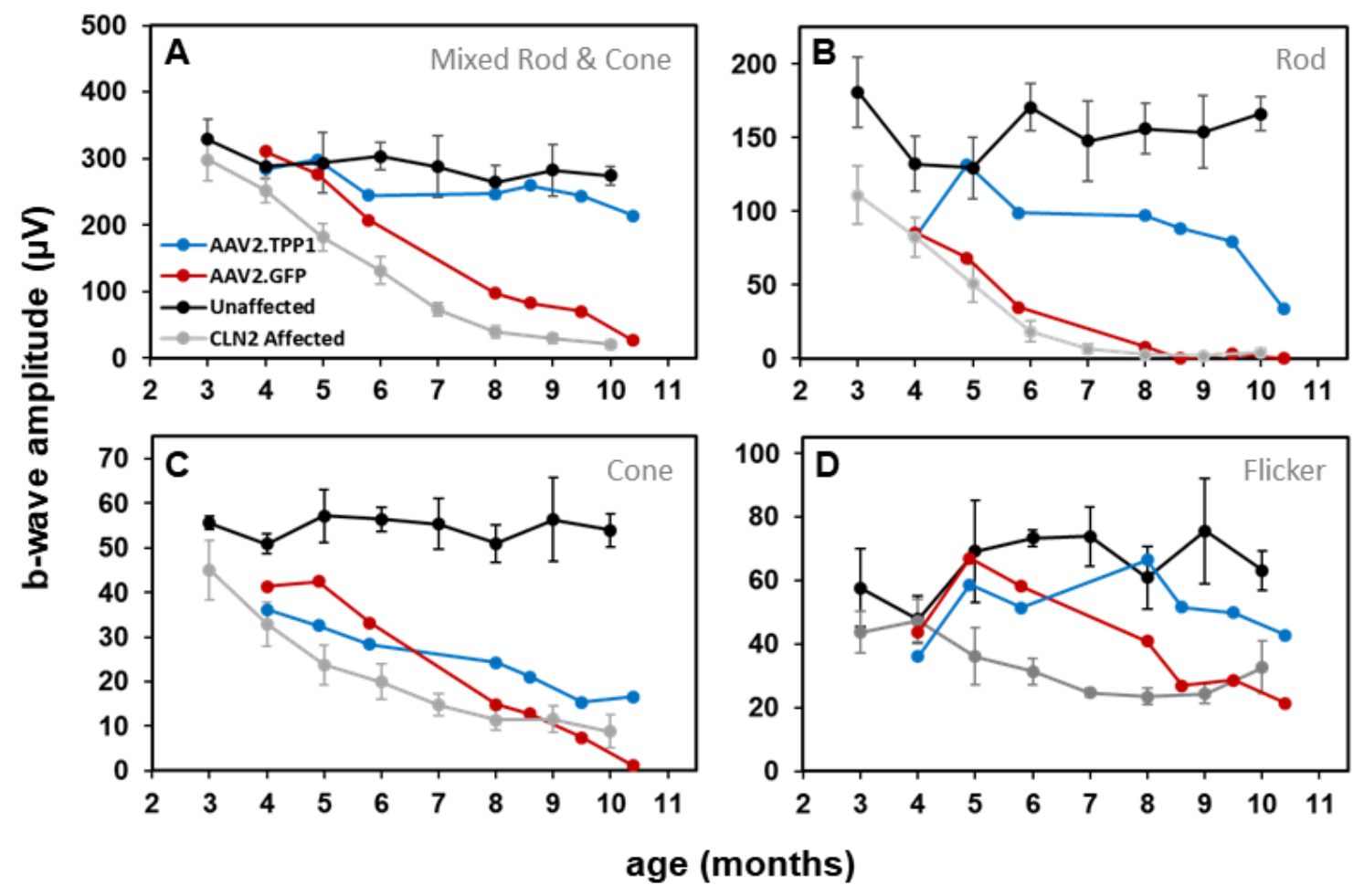

Figure 4-11: Mixed rod and cone (A), pure rod (B), pure cone $(C)$ and cone flicker (D) response amplitudes from dog $\mathrm{G}$. Black and gray traces represent historical data \pm SEM from normal $(n=7)$ and CLN2-affected $(n=6)$ untreated dogs, respectively, from the same research colony.

The b-wave amplitudes from dog $\mathrm{H}$ declined with age in both eyes. There was no preservation of cone function in the TPP1-treated eye, but pure rod and mixed rod and cone b-wave amplitudes were elevated in the treated eye compared to the GFP-control eye throughout the study period (Figure 4-12). As with previous dogs, a dramatic decline in ERG amplitudes coincided with onset of intraocular inflammation in dog I and rebounded once inflammation began to resolve (Figure 4-13). Pure rod and mixed rod and cone b-wave amplitudes were decreased only slightly from baseline in the treated eye at 10 months of age while there was little effect of treatment on cone function. 


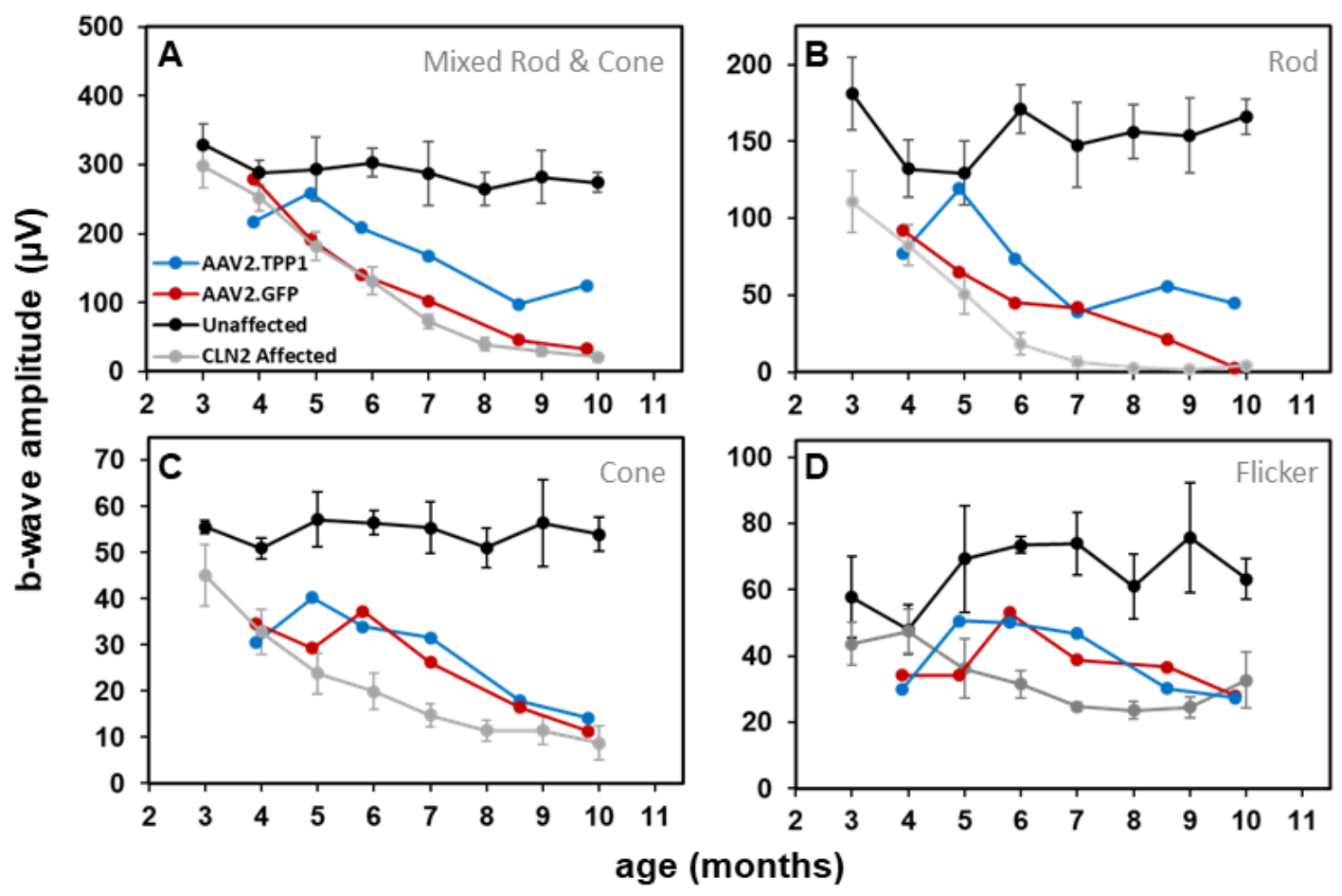

Figure 4-12: Mixed rod and cone (A), pure rod (B), pure cone $(C)$ and cone flicker (D) response amplitudes from dog $\mathrm{H}$. Black and gray traces represent historical data \pm SEM from normal $(n=7)$ and CLN2-affected $(n=6)$ untreated dogs, respectively, from the same research colony. 


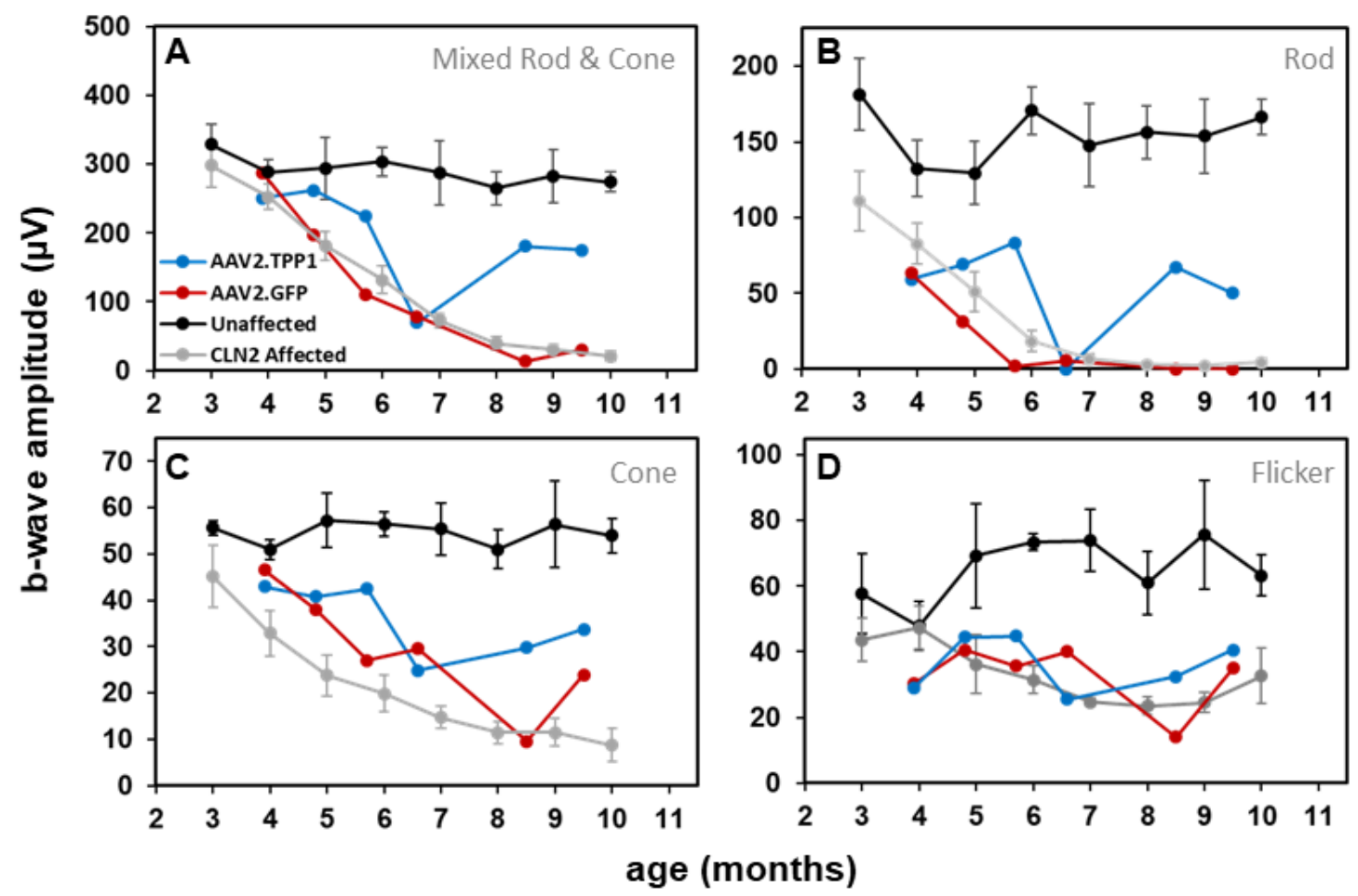

Figure 4-13: Mixed rod and cone (A), pure rod (B), pure cone (C) and cone flicker (D) response amplitudes from dog I. Black and gray traces represent historical data \pm SEM from normal $(n=7)$ and CLN2-affected $(n=6)$ untreated dogs, respectively, from the same research colony.

A single injection of AAV2.CAG.hTPP1 preserves retinal function for at least 6 months. Average mixed rod and cone and pure rod b-wave amplitudes were preserved in TPP1-treated eyes relative to control eyes through 10 months of age (Figure 4-14A and B). Pure cone b-wave amplitudes were significantly larger in treated eyes than control eyes at 10 months of age while there was no effect of treatment on cone flicker responses (Figure 4-14C and D). 


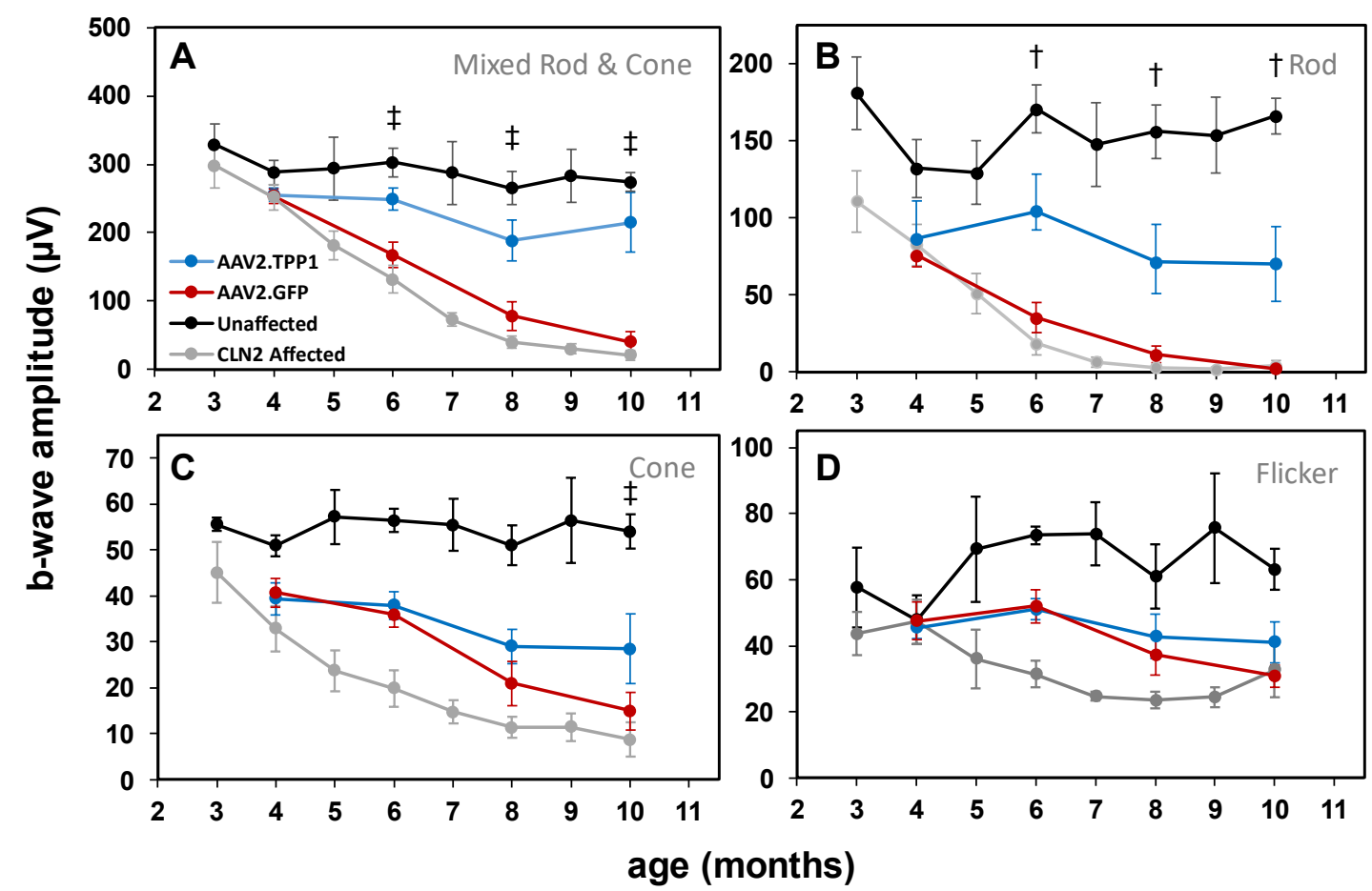

Figure 4-14: Average mixed rod and cone $(A)$, pure rod $(B)$, pure cone $(C)$ and cone flicker (D) b-wave amplitudes \pm SEM from dogs $E-I$. $\ddagger p \leq 0.001, \dagger p<0.01$. Black and gray traces represent historical data \pm SEM from normal $(n=7)$ and CLN2-affected $(n=6)$ untreated dogs, respectively, from the same research colony.

\subsection{Discussion}

The data presented here demonstrate that a single intravitreal injection of AAV2.CAG. $h T P P 1$ is effective in preserving retinal function in canine CLN2 disease and support further investigation to develop this approach to preserve retinal function in children affected by this disorder. Pure rod and mixed rod and cone responses were preserved at baseline levels until dogs reached end-stage neurological disease 6 months after treatment. Apparent cone function was also preserved, though to a lesser extent and it was not consistently preserved in all treated eyes. AAV2 has been shown to transduce rods more efficiently than 
cones in several species including dogs (Bennett et al., 1999; Vandenberghe et al., 2013, 2011). However, this seems unlikely to explain the difference as we have seen no evidence of any photoreceptor transduction, consistent with reports that vector delivery via intravitreal injection results in transduction primarily of the inner retina. Indeed, preliminary data indicates that GFP and hTPP1 expression were most pronounced in the inner retina. Some RPE cells, however, were heavily labeled while there was no detectable labeling in neighboring cells, again consistent with reports that at a high enough dose of AAV2 transduces RPE and photoreceptor cells but with much lower efficiency than cells of the inner retina. While intravitreal ERT was able to preserve both rod and cone function, both intravitreal TPP1-MSC implantation and gene therapy have preserved rod function much better than cone function, indicating that this discrepancy could be related to the smaller quantities of TPP1 delivered with these methods. The canine retina is rod-dominant and the area centralis, an area with high cone density, is located in the superior temporal retina (Beltran et al., 2014; Mowat et al., 2008). The AAV2 vector does not transduce every cell and, because of the angle of the needle during injection, transduces primarily the superior nasal retina. Perhaps this vector at this dose simply does not transduce enough cone bipolar or cone ganglion cells to effectively preserve cone function. If this is the case, it may be possible to improve the treatment by using a higher vector dose (which may cause more severe inflammation), altering the injection method such that the superior temporal retina is most strongly transduced or employing an 
AAV serotype with enhanced transduction efficiency with intravitreal administration.

Novel engineered capsids have been developed with improved transduction of outer retinal and RPE cells after intravitreal injection in mice (Dalkara et al., 2013; Trapani and Auricchio, 2018; Vandenberghe and Auricchio, 2012). However, the impressive results that have been seen in mice with these engineered capsids have not been replicated in large animals like dogs and monkeys and improved outer retinal transduction has been seen in these species only at doses which result in toxicity (Dalkara et al., 2013; Mowat et al., 2014; Ramachandran et al., 2017). It has become clear that transduction patterns in mice cannot be extrapolated to larger animals and that testing AAV vectors in large animals is a critical translational step. These novel capsids are not yet ready to be taken into the clinic, although further work may develop improved vectors for use in humans. In addition to improved transduction of the outer retina, novel vectors that allow for treatment with a smaller dose to minimize the risk of toxicity and inflammation and that avoid the issue of pre-existing antibodies would be extremely beneficial. However, to date, clinical trials have primarily employed AAV2 as does the only approved retinal gene therapy, which may expedite regulatory approval and be more attractive to potential sponsors (Trapani and Auricchio, 2018). In the present study, we saw a pronounced treatment benefit which supports further development of AAV2-mediated TPP1 gene therapy. Other modifications could improve the treatment, such as altering the injection strategy and reducing or preventing inflammation. 
One potential downside to an in vivo gene therapy approach is that repeated treatments are unlikely to be possible should the effects of the treatment wane or disappear over time due to the likelihood of a host immune response against the viral capsid. However, long-term follow-up data from clinical trials indicates that the benefit from AAV-mediated gene therapies can be long-lasting. Voretigene neparvovec, an AAV2-based gene therapy approved to treat the inherited retinal dystrophy Leber congenital amaurosis, has so far shown durable treatment effects up to 4 years after vector administration (Drack et al., 2019; Maguire et al., 2019). In the CNS, Onasemnogene abeparvovec, an AAV9-based gene therapy approved to treat spinal muscular atrophy, has shown durable effects up to 6.2 years after treatment (Mendell et al., 2021). In hemophilia, stable expression of the therapeutic product has been seen for up to 8 years in patients in a Phase 3 clinical trial of an AAV8-based gene therapy and for up to 10 years in dogs (Nathwani et al., 2018; Nguyen et al., 2021; Peyvandi and Garagiola, 2019). These encouraging results indicate that a single treatment can result in long-term efficacy, though it remains to be seen just how long the effects will last.

All dogs treated with the therapeutic dose exhibited inflammation in the TPP1-treated eye but not in the GFP-control eye, indicating that the inflammation is due to an immune response against the hTPP1 protein rather than the AAV2 vector. The earliest onset of inflammation seen in this study was 3 weeks posttreatment and occurred as late as 25 weeks post-treatment. This delay in inflammation onset is more consistent with a ramping up of hTPP1 expression and continued production of the protein than with capsid concentration which 
would decline over time. An immune response against the hTPP1 protein is consistent with previous studies where dogs treated with intravitreal and intrathecal rhTPP1 protein and with intrathecal gene therapy produced anti-TPP1 antibodies (Katz et al., 2015; Vuillemenot et al., 2015; Whiting et al., 2020b, 2020a). As discussed previously, it is likely that the immune response against hTPP1 seen in these dogs will be much less of a concern in human CLN2 patients. The dog eye may be particularly susceptible to immune-mediated uveitis immunogenicity of human proteins is frequently greater in pre-clinical models than in humans (Ponce et al., 2009). Additionally, the Dachshund CLN2 model represents a worst-case scenario as the frameshift mutation predicts a premature stop codon which results in a lack of TPP1 production. In humans, some CLN2 variants are nonsense mutations while many others result in production of a nonfunctional TPP1 protein ("NCL Mutation and Patient Database," n.d.). Finally, immune responses against CSF-infused rhTPP1 seen in treated CLN2-affected dogs have not been an issue in children receiving the treatment (Katz et al., 2014; Vuillemenot et al., 2015, 2011). Although some children who received CSF infusions of rhTPP1 developed anti-TPP1 antibodies, this was not associated with severe hypersensitivity reactions or with decreased treatment efficacy (Cherukuri et al., 2018).

In human patients, a greater concern is likely to be the risk of an anti-AAV immune response. Immune responses in human subjects who have received AAV-mediated gene therapy have been primarily against the viral capsid, including neutralizing anti-AAV antibodies and anti-AAV T cell responses 
(Bainbridge et al., 2015; Cukras et al., 2018; Heier et al., 2017; Vandamme et al., 2017). It is very encouraging that this dose was therapeutically effective and not associated with vector toxicity. The highest dose was administered a month earlier than the three lower doses, so we could consider repeating the second highest dose at the same age as the high dose to see if a lower dose could be effective if administered at a younger age. These dogs are housed in a laboratory environment and may not be exposed to wild-type AAV2, whereas pre-existing anti-AAV antibodies are a concern in human gene therapy trials. Efforts to develop ELISA methods to detect anti-TPP1 and anti-AAV2 total binding antibodies in order to explore the immune response in more detail are ongoing. It would also be valuable to determine if there is a T cell response, as a better understanding of the nature of the immune response would help us fine-tune our immunosuppressive protocol, the focus of our current efforts on this project. It was surprising that dogs on immunosuppressive doses of both cyclosporine (which reduces $\mathrm{T}$ cell function by inhibiting calcineurin and blocking cytokine production) and leflunomide (which inhibits pyrimidine synthesis and inhibits both $\mathrm{T}$ and $\mathrm{B}$ cell proliferation) exhibited relatively low $\mathrm{T}$ cell suppression and indicates that closer monitoring of not just the blood levels of these drugs but of $T$ cell suppression is warranted (Archer et al., 2014; Breedveld and Dayer, 2000).

The results of this study emphasize that controlling intraocular inflammation is critical to fully demonstrate preclinical efficacy and to improve the utility of canine models for preclinical testing of ocular therapies. We and others have observed that the ERG may be attenuated during an inflammatory episode 
(Brouwer et al., 2020). This is likely due at least in part to vitreal opacity and possibly due as well to inflammation of retinal vasculature, since we saw perivascular cuffing around inner retinal vessels in rhTPP1-treated eyes in Chapter 2 and retinal vasculitis impairs vision (Ku et al., 2012). Corneal and lens opacity and miosis secondary to synechia likely also contributed in some cases and should be prevented. As the ERG is our readout of retinal function, inflammation must be controlled in order to accurately assess the efficacy of the treatment. Dogs are common pre-clinical models, so a method to prevent or mitigate intraocular inflammation would improve the usefulness of the species for testing ocular therapeutics.

In upcoming studies, we will continue to optimize our approach to minimize inflammation. The ultramicronized cyclosporine formulation that appears to have been beneficial to dog $\mathrm{G}$ will be used and a pharmacodynamic assay will be employed to assess immunosuppression and adjust dosing rather than the standard pharmacokinetic assay we have used with previous dogs. Because monthly intravitreal injections of triamcinolone appear to have been well tolerated in dogs $\mathrm{H}$ and $\mathrm{I}$, we will consider using this from the outset rather than alternating with sub-Tenon's injections which likely do not as effectively reach the retina. We will also test oral prednisone, which has been used in human retinal gene therapy trials, albeit primarily to prevent a reaction to the capsid immediately following vector administration. Hopefully, these modifications will be sufficient to prevent or at least greatly reduce treatment-related inflammation. 
A recent study tested intravitreal self-complementary AAV9.CLN5 and AAV9.CLN6 gene therapy in ovine models of CLN5 and CLN6 disease (Murray et al., 2021). In the sheep, a dose of $4 \times 10^{11} \mathrm{vg}$ was safe and well-tolerated. Even though no anti-inflammatory treatment was used, only one of six treated sheep developed uveitis one week after treatment and this resolved within a week. However, eye health was only monitored for three weeks after treatment. While this pilot study included only very limited ERG assessment, there was a pronounced treatment effect in AAV9. CLN5 treated eyes but no effect in AAV9.CLN6 treated eyes. This proof-of-principle study supports the idea that canine eyes may be particularly susceptible to inflammation and, taken together with our data, supports further development of retinal gene therapies for the treatment of retinal dysfunction in the NCLs. 


\section{Characterization of Visual System Pathology in a Canine Model of CLN5 Neuronal Ceroid Lipofuscinosis}

\subsection{Introduction}

The neuronal ceroid lipofuscinoses (NCLs) are a group of inherited progressive neurodegenerative disorders characterized by accumulation of autofluorescent lysosomal storage bodies in neurons and other cell types. Variants in 14 genes have been associated with different forms of NCL (BeckWödl et al., 2018; Butz et al., 2020). The CLN5 form of the disease is associated with sequence variants in CLN5 which encodes a lysosomal protein whose function remains unclear (De Silva et al., 2015; Jules et al., 2017; Larkin et al., 2013; Lebrun et al., 2009; Leinonen et al., 2017; Mamo et al., 2012; Moharir et al., 2013; Schmiedt et al., 2010). 37 pathogenic variants have been reported in all 4 exons and in noncoding regions of CLN5 ("NCL Mutation and Patient Database," n.d.; Savukoski et al., 1998). In most cases, the onset of clinical signs in CLN5 disease occurs in children between 2 and 8 years of age. Cognitive, motor and language function usually decline first followed by seizures and impaired vision progressing to blindness. However, there is some variability in visual signs between patients with different CLN5 disease alleles. In most patients, visual failure occurs between 5 and 10 years of age (Bessa et al., 2006; Pineda-Trujillo et al., 2005; Santavuori et al., 1982; Simonati et al., 2017; Xin et al., 2010), although a subset of affected individuals with certain variants exhibits visual signs that are minimal or begin later in the disease course (Ge et al., 2018; 
Xin et al., 2010). Vision loss in CLN5 disease has been attributed to a progressive decline in retinal function and associated retinal degeneration (Åberg et al., 2011). However, because CLN5 disease is characterized by widespread brain atrophy, central nervous system degeneration is also likely to play a significant role in vision loss.

Progress in understanding the pathogenesis of and developing treatments to prevent visual decline in CLN5 disease is likely to come from studying disease progression in large animal models. Naturally occurring CLN5 disease has been identified in Golden Retrievers, Border Collies and Australian Cattle Dogs as well as in Borderdale sheep and Devon cattle (Frugier et al., 2008; Gilliam et al., 2015; Houweling et al., 2006; Kolicheski et al., 2016; Melville et al., 2005). CLN5affected Golden Retrievers with a predicted truncating mutation in CLN5 develop progressive neurological signs as early as 13 months of age with seizures and visual impairment starting at 18 months of age. In the Golden Retriever disorder, previous documentation of functional visual impairment consisted primarily of observations by the dogs' owners; no objective assessments of retinal or visual function were performed. The affected companion dogs were euthanized due to disease progression at 30 to 34 months of age, 1.5 to 2 years after onset of clinical signs. Postmortem examination of the retinas revealed accumulations of autofluorescent storage bodies in several layers of the neural retina (Gilliam et al., 2015). In order to better characterize visual system pathology in this disorder, five Golden Retriever puppies homozygous for the previously identified CLN5 disease allele were evaluated for progression of retinal and brain pathology and 
changes with age in electroretinogram (ERG) and visual evoked potential (VEP) responses. A goal was to obtain baseline data that could be used in the design of potential therapeutic intervention studies.

\subsection{Methods}

\subsubsection{Animals}

Five CLN5-affected Golden Retriever puppy littermates were evaluated in this study. The puppies were homozygous for the CLN5:c.934_935delAG variant previously identified in the breed that predicts a truncated protein lacking the 39 C-terminal amino acids (Gilliam et al., 2015). The dogs were maintained on a 12:12 daily light cycle in AALAC-accredited facilities at the University of Missouri - Columbia. They received routine husbandry and veterinary care and were socialized outside of their pens daily. The study was performed in accordance with the U.S. National Research Council Guide for the Care and Use of Laboratory Animals and was approved by the University of Missouri Animal Care and Use Committee.

\subsubsection{Electrophysiology and Retinal Imaging}

Complete ophthalmic exams were performed at $6.5,15.5$ and 17.5 months of age as described in section 2.2.2. Electroretinogram (ERG) recordings were performed at approximately $7,13,16,20$ and 23 months of age as described in section 2.2.5. Dogs were sedated with up to $30 \mu \mathrm{g} / \mathrm{kg}$ intramuscular dexmedetomidine and $0.25 \mathrm{mg} / \mathrm{kg}$ intravenous midazolam for ERG recordings. Visual evoked potential (VEP) recordings were performed immediately after the 
ERG. Room lights were turned on and needle electrodes were placed under the skin, with the active electrode placed over the rostral aspect of the occipital protuberance, the ground halfway between the lateral canthus and the base of the ear, and the reference on the dorsal aspect of the snout. The VEP protocol consisted of thirty 12.65 photons $/ \mathrm{cm} 2 / \mathrm{s}(3 \mathrm{~cd} / \mathrm{m} 2)$ bilateral flashes 1 second apart, and this was repeated three times. VEP data were analyzed in ERGView. A $100 \mathrm{~Hz}$ lowpass filter was applied and the latency and amplitude of peaks P1, N1, P2, N2 and P3 were measured as shown in Figure 5-1 (Kimotsuki et al., 2005; Strain et al., 1990). Latencies were measured as the time elapsed between the stimulus and each peak and amplitudes were measured from peak to peak ( 0 ms-P1, P1-N1, N1-P2, P2-N2 and N2-P3).

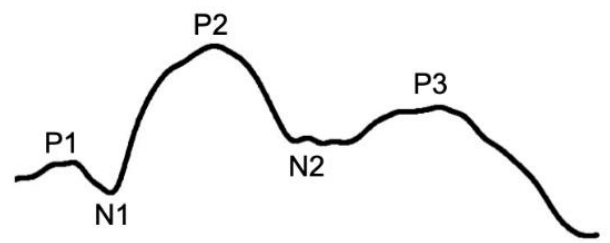

Figure 5-1: Elements of the VEP indicating the three positive peaks (P1, P2, and $\mathrm{P} 3$ ) and the two negative peaks (N1 and N2) that are typically present in normal healthy dogs when the recordings are performed as described for the present study (Strain et al., 1990).

Retinal imaging was performed immediately following each ERG and VEP recording session as described in section 2.2.6.

\subsubsection{Histology}

The five dogs evaluated in this study were euthanized at the ages indicated in Table 5-1. Each euthanasia procedure was performed between 5 and 6 hours 
after the onset of the light phase of the daily light cycle. Shortly after the last retinal imaging procedure was completed, the dogs were euthanized via intravenous infusion of pentobarbital $(15 \mathrm{ml}$ of $390 \mathrm{mg} / \mathrm{ml}$ pentobarbital FatalPlus solution, Dearborn, MI). Immediately after euthanasia, the left eyes were enucleated and immersed in $2.5 \%$ glutaraldehyde in $0.1 \mathrm{M}$ sodium cacodylate buffer at $\mathrm{pH} 7.4$ and the right eyes were enucleated and immersed in 3.5\% depolymerized paraformaldehyde, $0.05 \%$ glutaraldehyde, $120 \mathrm{mM}$ sodium cacodylate, $1 \mathrm{mM} \mathrm{CaCl}$, pH 7.4 (immuno fixative). Both eyes were enucleated with approximately $1 \mathrm{~cm}$ of proximal optic nerve attached. Within minutes of being immersed in the fixatives, the corneas, irises and lenses were removed from the eyes, and fixative solution was injected into the vitreous. The eyecups with optic nerves were then incubated in the fixatives with gentle mixing at room temperature for a minimum of 24 hours before further dissections were undertaken.

\begin{tabular}{ccc}
\hline Dog & Sex & $\begin{array}{c}\text { Age at Euthanasia } \\
\text { (months) }\end{array}$ \\
\hline Dog V & F & 20.1 \\
\hline Dog W & F & 21.9 \\
\hline $\operatorname{Dog}$ X & F & 23.1 \\
\hline Dog Y & F & 23.4 \\
\hline Dog Z & M & 23.4 \\
\hline
\end{tabular}

Table 5-1: Ages at which dogs were euthanized 
After the initial fixation, the optic nerves were dissected from each eye and transferred to small vials of the same fixative used for the initial fixation. The eyecups were then dissected into 8 regions as shown in Figure 5-2 and described in section 2.2.8. All microscopic analyses were performed on sections obtained from the regions of the posterior retina indicated in Figure 5-2. A segment of each immuno-fixed optic nerve was embedded in paraffin and sectioned at a thickness of $5 \mu \mathrm{m}$. Sections were stained with Masson's Trichrome. A sample of posterior retina from each immuno-fixed eye was embedded in cryo-embedding medium and cryostat sectioned as described in section 4.2.2. The sections were mounted on Superfrost Plus slides (Fisher Scientific, Waltham, MA) and were examined for NCL storage body-specific autofluorescence as described previously (Katz and Redmond, 2001).

A second sample of posterior retina from each immuno-fixed eye was washed in $170 \mathrm{mM}$ sodium cacodylate $(\mathrm{pH} 7.4)$ and embedded in paraffin. Sections of the paraffin-embedded retina samples along with sections of the optic nerves were cut at a thickness of $5 \mu \mathrm{m}$ and were immunostained for localization of lysosome-associated membrane protein 2 (LAMP2). The primary antibody used for immunostaining was anti-LAMP2A (1:300; Abcam, catalogue \# ab18528, Cambridge, UK). Immunostaining was performed as described previously (Morgan et al., 2014).

The corresponding regions of the retinas from the eyes fixed in $2.5 \%$ glutaraldehyde were postfixed in osmium tetroxide and embedded in epoxy resin as described in section 2.2.8. Sections of these samples were cut at a thickness 
of $0.6 \mu \mathrm{m}$ for light microscopy and 70 to $90 \mathrm{~nm}$ for electron microscopy. Sections for light microscopic examination were stained with Toluidine blue and sections for electron microscopic examination were mounted on thin-barred copper grids and stained with uranyl acetate and lead citrate. Light microscopy was performed using a Leica DMI 6000B microscope, and electron microscopy was performed with a JEOL JEM-1400 transmission electron microscope equipped with a Gatan digital camera.

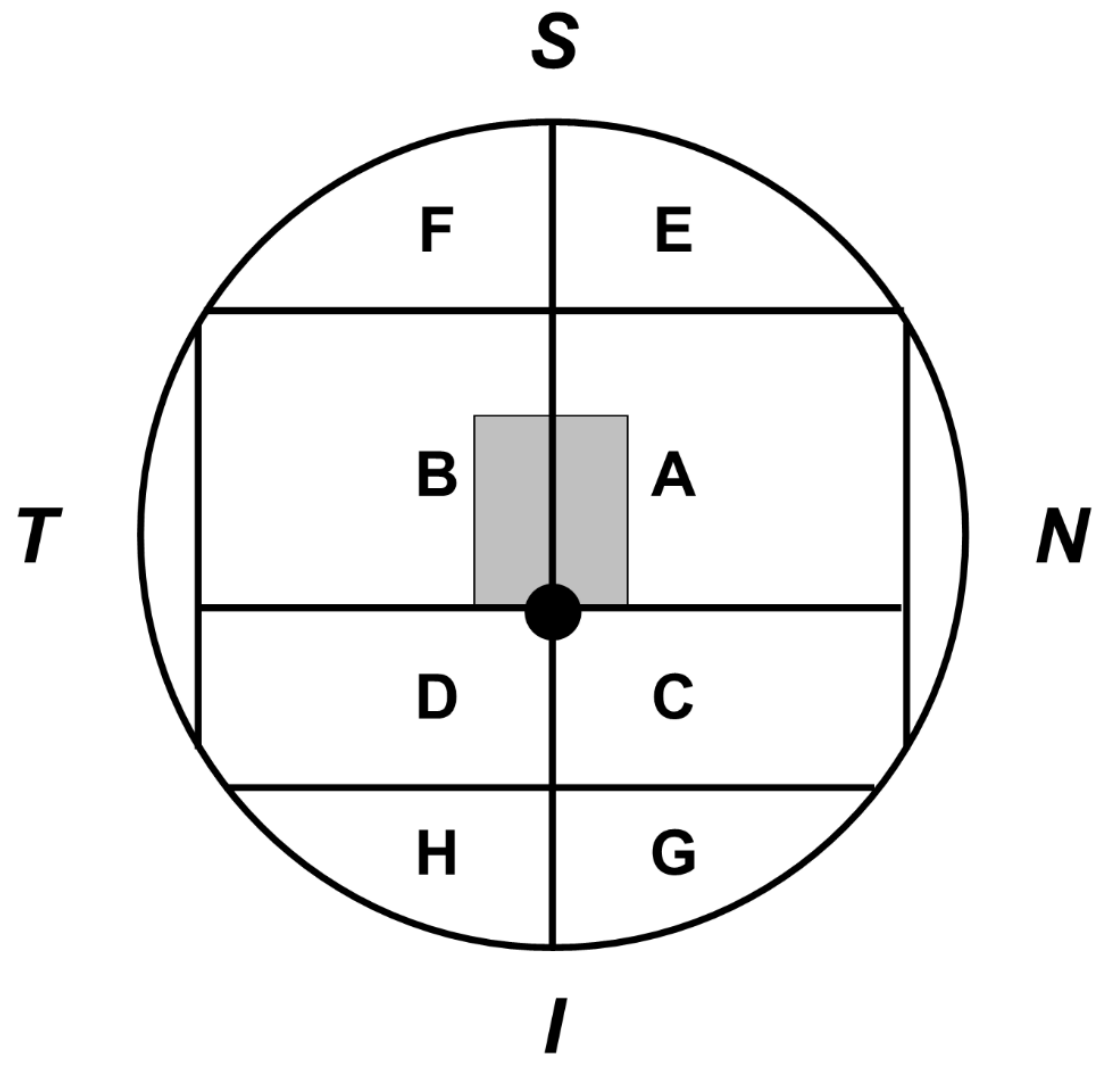

Figure 5-2: Diagram illustrating the eye dissections. N:nasal; T:temporal; S:superior; I:inferior. Black circle: optic nerve head. The eyecups were dissected into the regions indicated by $A-G$. For this study, microscopic analyses were performed on the region of the retina indicated by the shaded rectangle. 
Immediately after the eyes were enucleated the brain was collected from each dog and immersed in ice-chilled PlasmaLyte (Baxter Healthcare, Deerfield, IL) for 10 minutes. The brain was then cut into $4 \mathrm{~mm}$ thick coronal slices using an Adult Rhesus monkey brain matrix (Electron Microscopy Sciences, Hatfield, PA). Half of each brain slice was fixed in $2.0 \%$ glutaraldehyde, $1.12 \%$ paraformaldehyde, $130 \mathrm{mM}$ sodium cacodylate, $1 \mathrm{mM} \mathrm{CaCl} 2, \mathrm{pH} 7.4$ and the other half in immuno fixative. Slices of occipital cortex were dissected from these samples and processed for and examined with fluorescence microscopy, immunohistochemistry (IHC), and electron microscopy as described above for the retina samples.

\subsubsection{Visually Mediated Behavior}

Beginning at 21 months of age, a visual tracking test was performed on dogs $\mathrm{W}, \mathrm{X}, \mathrm{Y}$ and $\mathrm{Z}$ as part of routine neurologic examinations. The test consisted of a cotton ball dropped from approximately eye-level height in front of the dog. This was repeated approximately 5-10 times per evaluation. The dog's ability to track the cotton ball on its path from point of drop to the floor was assessed subjectively by watching eye fixation and head movements in relation to the falling cotton ball. Visual tracking ability at each assessment was assigned one of the following designations: good (consistently tracks cotton ball well), decreased (inconsistently tracks cotton ball from point of drop to floor), poor (occasionally reacts when cotton ball contacts floor but does not track path from drop to floor), or absent (does not track cotton ball at all). Cotton ball vision tests were repeated every 1-3 weeks at time of neurologic examinations. Vision was 
also subjectively evaluated at time of neurologic examinations through simple observation of behavior and ability to navigate around obstacles in a closed room.

\subsubsection{Statistical Analyses}

All statistical tests were performed using SigmaPlot (Systat Software Inc., San Jose, CA). Normality was confirmed with the Shapiro-Wilk test. A two-tailed Student's t-test was used to compare ERG b-wave amplitudes, a-wave amplitudes and b:a wave ratios at 7 months and 22-23 months of age. A twotailed Student's t-test was also used to compare VEP P2 amplitudes and the ratio of P2 amplitudes to cone b-wave amplitudes between 7 months and 22-23 months of age and between 7, 12, 16 and 20 months of age combined and 22-23 months of age. Statistical power analyses indicated that sample sizes were too small to enable reliable pairwise comparisons of data between each of the timepoints at which ERG and VEP data were obtained.

\subsection{Results}

\subsubsection{Ocular Imaging}

Ophthalmic exams were performed at $6.5,15.5$ and 17.5 months of age. Pupillary light reflex and menace response were intact through 17.5 months of age but were not able to be assessed at later ages due to behavioral changes and lack of compliance with examination. Dogs $W$ and $Z$ developed 1 to 3 small bullous retinal detachments per eye, first noted at 15.5 months of age on ophthalmic exam and 20 months of age by SLO imaging, respectively. In both 
cases, lesions were confirmed with OCT imaging (Figure 5-3F and 5-3G). No other abnormalities were observed.
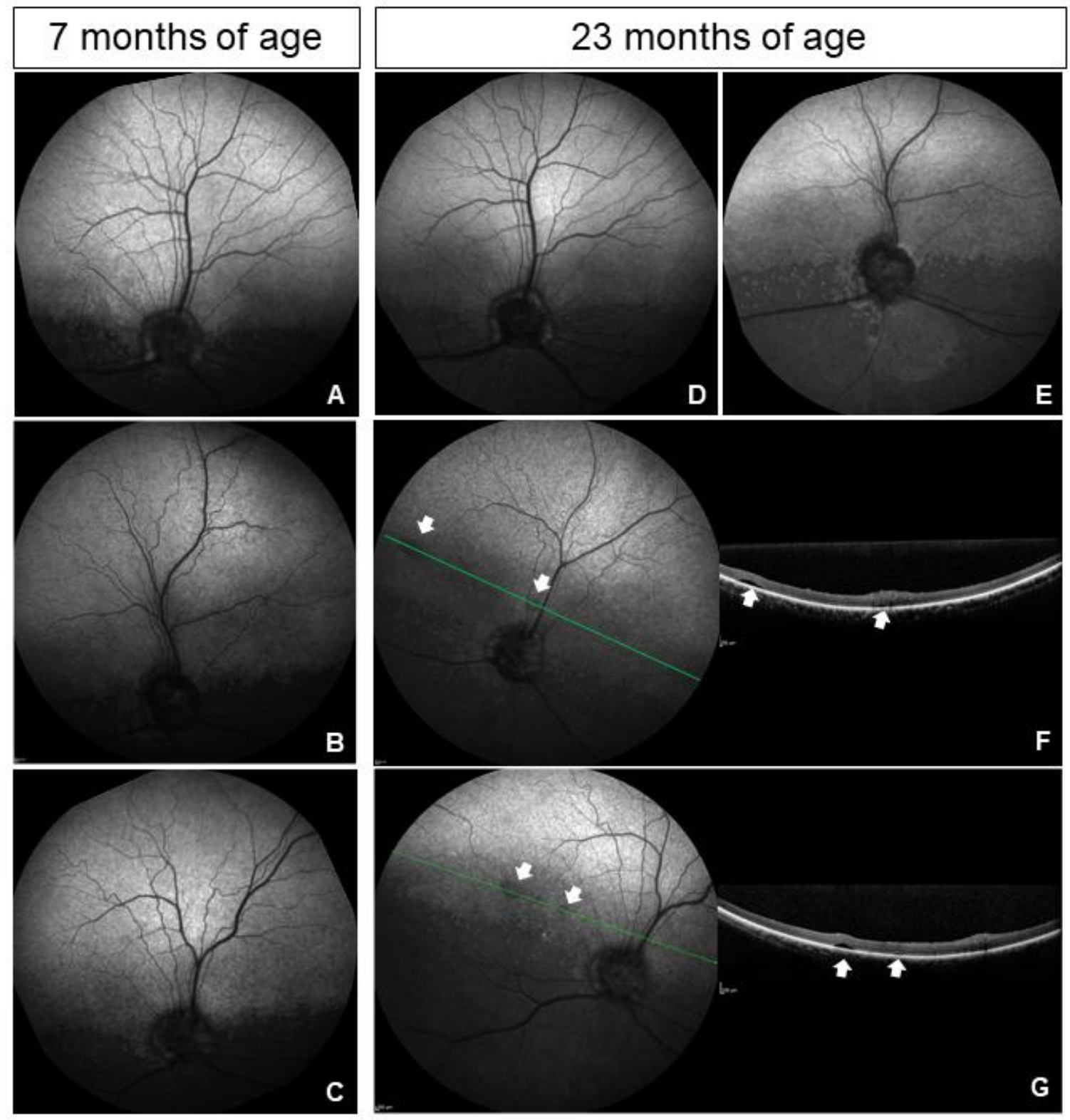

Figure 5-3: SLO and OCT images of dogs $X(A), Y(B)$ and $Z(C)$ at 7 months of age and dogs $X(D), Y(E), W(F)$ and $Z(G)$ at 23 months of age. 1-3 small bullous retinal detachment lesions were present in both eyes of dog $W(F)$ and $\operatorname{dog} Z(G)$ by 23 months of age. Green lines indicate the locations from which the OCT images were obtained. Arrows indicate the locations of the retinal lesions. 


\subsubsection{Electroretinographic and Visual Evoked Potential Findings}

The scotopic b-wave response amplitudes elicited by a low-intensity flash after 20 minutes of dark adaptation did not change significantly between 7 and 23 months of age (Figure 5-4A, $p=0.263$ ). The $b$-wave amplitudes elicited by scotopic high intensity flash stimuli at 23 months were an average of 53\% lower than the responses to the same stimuli elicited at 7 months of age (Figure 5-4B, $p=0.004$ ) while the a-wave amplitudes were unchanged (Figure $5-4 C, 0.231$ ), resulting in a decrease in the $b$ - to a-wave ratio (Figure 5-4D, $p=0.005)$. The $b-$ wave amplitudes elicited by both photopic single flash (Figure 5-4E) and flicker (Figure 5-4H) stimuli were decreased at 23 months relative to the responses elicited by these stimuli at 7 months of age $(p=0.006$ and 0.029 for the flash and flicker responses respectively). Photopic a-wave amplitudes elicited by the single flash were also reduced by 23 months (Figure $5-4 F, p=0.039$ ) as was the $b$-wave to a-wave ratio (Figure $4 \mathrm{G}, \mathrm{p}=0.042$ ), indicating that reductions in the $\mathrm{b}$-wave were greater than the decreases in a-wave amplitudes. Representative ERG responses of $\operatorname{dog} X$ at 7, 16 and 23 months of age are shown in Figure 5-5. 

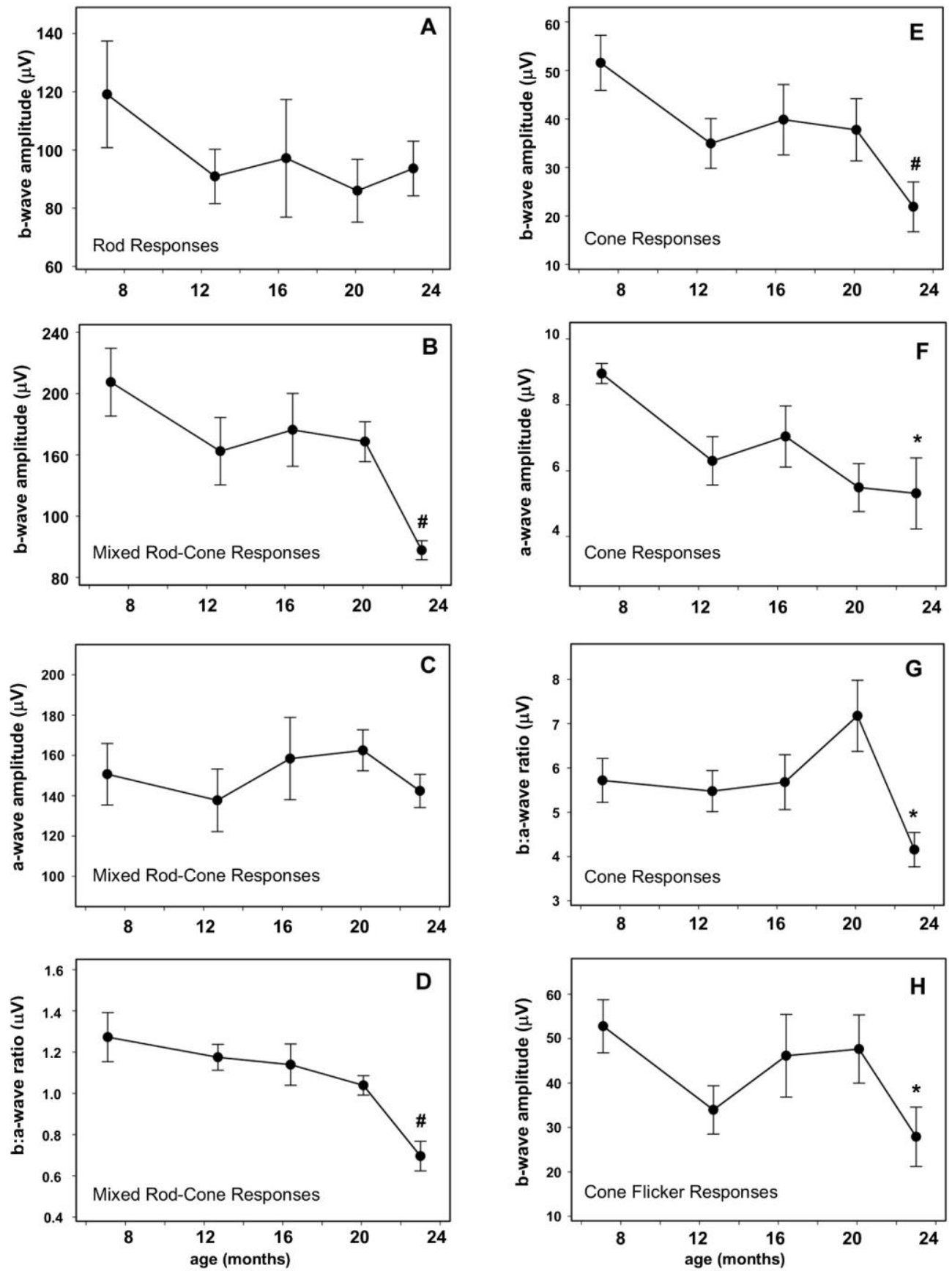

Figure 5-4: Mean ERG response amplitudes $\pm S E M$ for all 5 dogs. Cone function was primarily affected. Mean cone $(\# p<0.01, E)$, cone flicker $\left({ }^{*} p<0.05, H\right)$ and mixed rod and cone $(\# p<0.01, B) b$-wave amplitudes were significantly reduced from baseline at 23 months of age. The mean cone a-wave amplitude $(F)$ was significantly decreased at 23 months of age $\left({ }^{*} p<0.05\right)$ while the mixed rod and cone a-wave $(C)$ was unchanged. Mean cone $\left({ }^{*} p<0.05, G\right)$ and mixed rod and cone $(\# p<0.01, D) b: a$ wave ratios were significantly decreased from baseline at 23 months of age. Mean rod response (A) was not significantly changed from baseline at 23 months of age. 


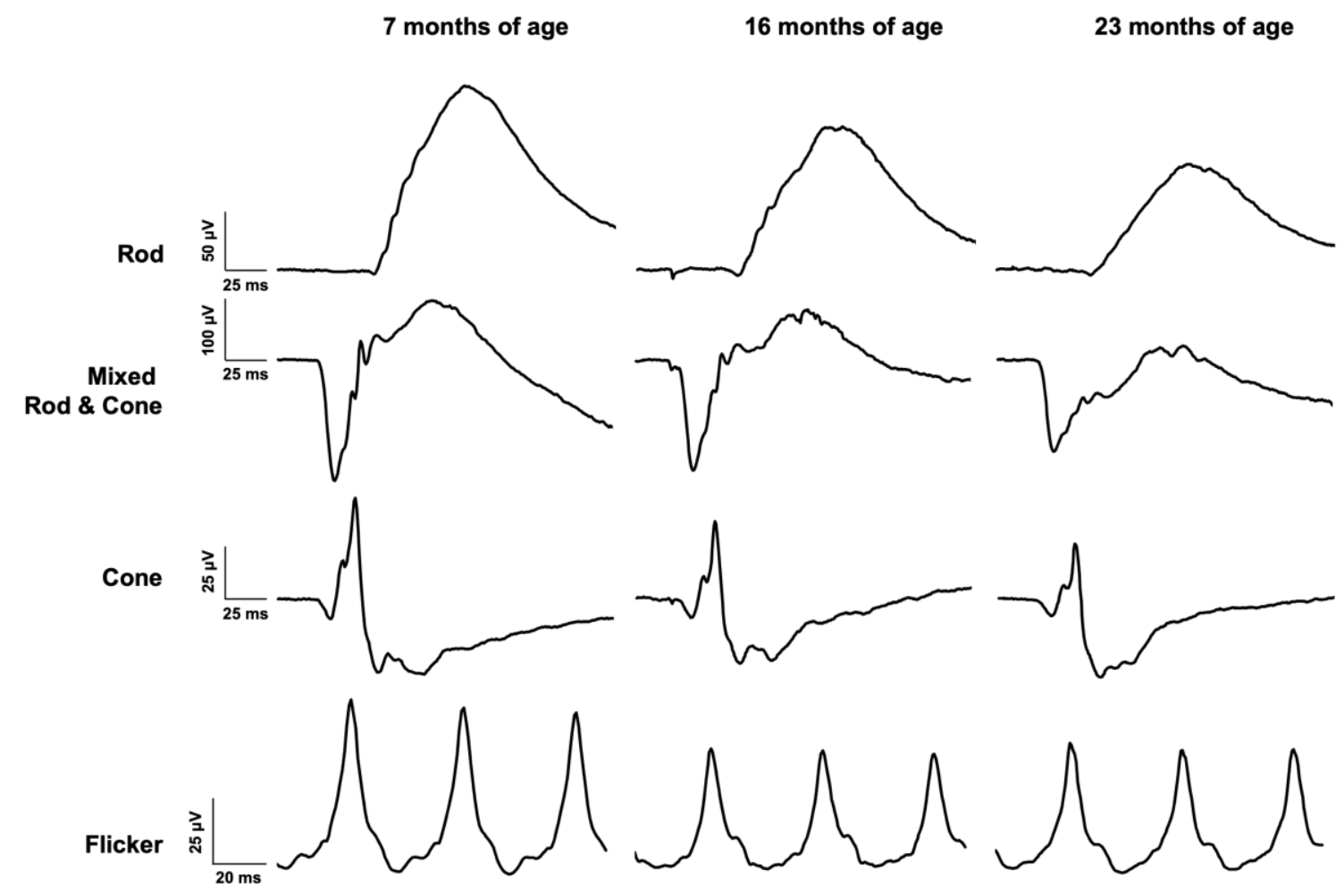

Figure 5-5: ERG waveforms from dog $X$ at 7, 16 and 23 months of age.

For the VEPs, the latencies and amplitudes of peaks P1, N1, N2 and P3 and the latencies of P2 did not change significantly with age (data not shown). The amplitude of P2 was reduced at 23 months of age relative to 7 months of age $(p=0.042)$ and to amplitudes at $7,12,16$ and 20 months combined $(p=0.016)$ (Figure 5-6A). Because the VEP was elicited with a bright flash stimulus under photopic conditions and therefore mediated primarily by cones, we also evaluated the ratio of the P2 amplitude to the cone b-wave amplitude (Figure 5$6 B)$. This ratio was increased at 23 months compared to both 7 months $(p=0.038)$ and all other time points combined $(p=0.027)$. Some of the VEP responses 
included one or more positive peaks following P2 (asterisks in Figure 5-7) that do not appear in the VEP responses of normal healthy dogs (Strain et al., 1990). These peaks were more pronounced at the later ages. VEP waveforms from dogs $Y$ and $Z$ at 7, 16 and 23 months of age are shown in Figure 5-7.
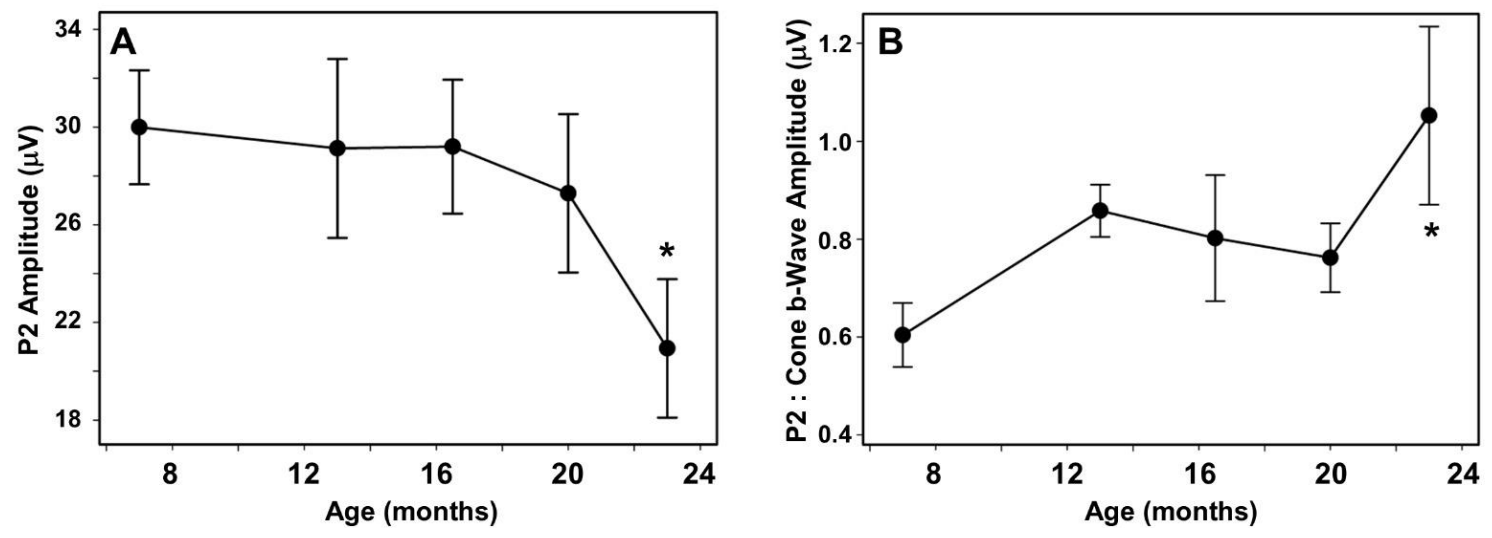

Figure 5-6: Mean VEP P2 amplitudes \pm SEM for all 5 dogs (A) and the mean ratio of the P2 amplitude to the cone b-wave amplitude \pm SEM for all 5 dogs (B). The mean amplitude of P2 was decreased by 23 months of age ( $\left.{ }^{*} \mathrm{p}<0.05\right)$. The mean ratio of the $\mathrm{P} 2$ amplitude to the cone $b$-wave amplitude was increased by 23 months $\left({ }^{*} p<0.05\right)$.
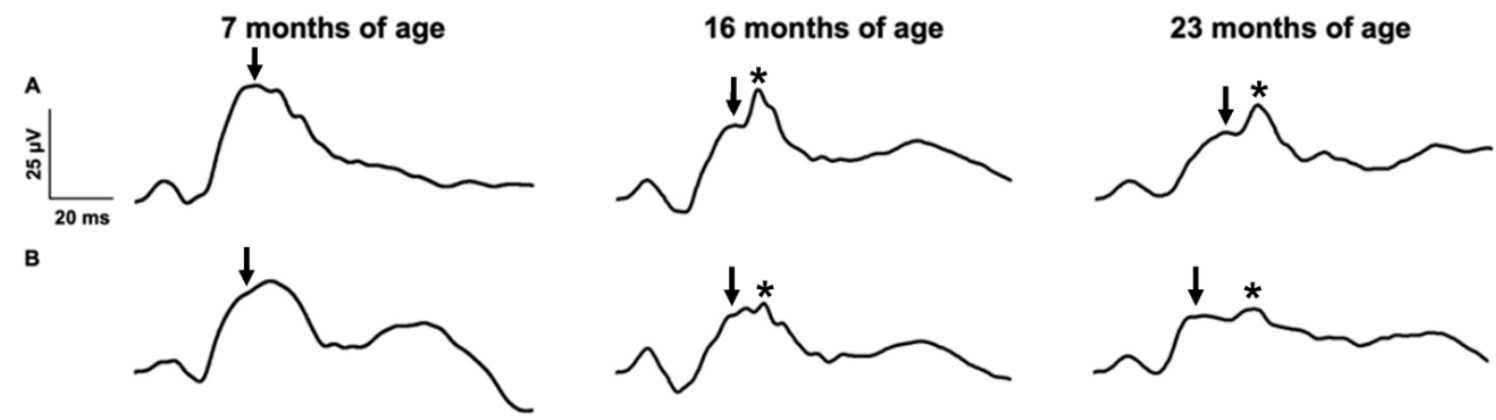

Figure 5-7: VEP waveforms from $\operatorname{dog} \mathrm{Y}(\mathrm{A})$ and $\operatorname{dog} \mathrm{Z}(\mathrm{B})$ at 7,16 and 23 months of age. Arrows indicate P2. P2 was identified as the first positive peak following N1, following the standard convention. In some of the recordings, there was an additional positive peak following P1 (asterisks) that has not been reported in normal dogs. 


\subsubsection{Visually Mediated Behavior}

Visually mediated behavior was assessed in part by determining whether the dogs visually tracked falling cotton balls that were dropped in front of them from above the dog's head level. The assessments were performed at periodic intervals on four of the dogs starting at 21 months of age (the fifth dog was euthanized prior to the start of these evaluations). Tracking behavior was rated as good, decreased, or poor relative to the tracking by healthy dogs under the same conditions. For consistency, all ratings were performed by the same observer. The findings are summarized in Table 5-2. All of the dogs exhibited decreased visual tracking with disease progression. At the first cotton ball test performed at 21 months of age, two dogs were assessed to have good visual tracking ability and two dogs had decreased visual tracking ability. Subsequent evaluations showed progressive decline of ability to track the cotton ball for all dogs.

\begin{tabular}{lccccc} 
& \multicolumn{5}{c}{ Age (months) } \\
\cline { 2 - 5 } Dog & 21.0 & 21.9 & 22.4 & 23.0 & 23.2 \\
\hline Dog W & Decreased & Poor & & & \\
\hline Dog X & Good & Good & Decreased & Decreased \\
\hline Dog Y & Decreased & Poor & Poor & Poor & Poor \\
\hline Dog Z & Good & Decreased & Poor & Poor & Poor \\
\hline
\end{tabular}

*If no data, dogs were euthanized prior to this age.

Table 5-2: Cotton ball visual tracking assessments 
Visual deficits in navigation ability in a closed room became apparent starting at 21.0 to 22.4 months of age in dogs $\mathrm{W}, \mathrm{X}, \mathrm{Y}$ and Z. Behavioral visual deficits subjectively worsened over variable periods of time, with dogs $X, Y$ and $Z$ having markedly progressed visual impairment by 22.4 to 23.1 months of age. Dog W's visual deficits were subjectively less progressed by the time of euthanasia at 21.9 months of age.

\subsubsection{Retinal and Occipital Cortex Morphology}

Despite the decreased retinal and visual function, the overall morphology of the retinas remained relatively normal, even at 23.4 months of age (Figure 5-8). Inclusions with autofluorescence properties typical of ceroid and lipofuscin were observed in all retinal layers except the photoreceptor outer segments (Figure 59). The accumulation of these inclusions was most pronounced in the retinal ganglion cell bodies, along the outer limiting membrane, and in the retinal pigment epithelium (RPE). The accumulation of this autofluorescent material was already pronounced in the dog euthanized at 20 months of age and was not appreciably different in the dogs euthanized at later time points. In the occipital cortex, by 20 months of age almost every neuron contained significant amounts of autofluorescent storage material (Figure 5-10). The autofluorescent inclusions co-localized with immunostaining for the lysosomal marker LAMP2 in both the retina and the occipital cortex (Figure 5-11). 


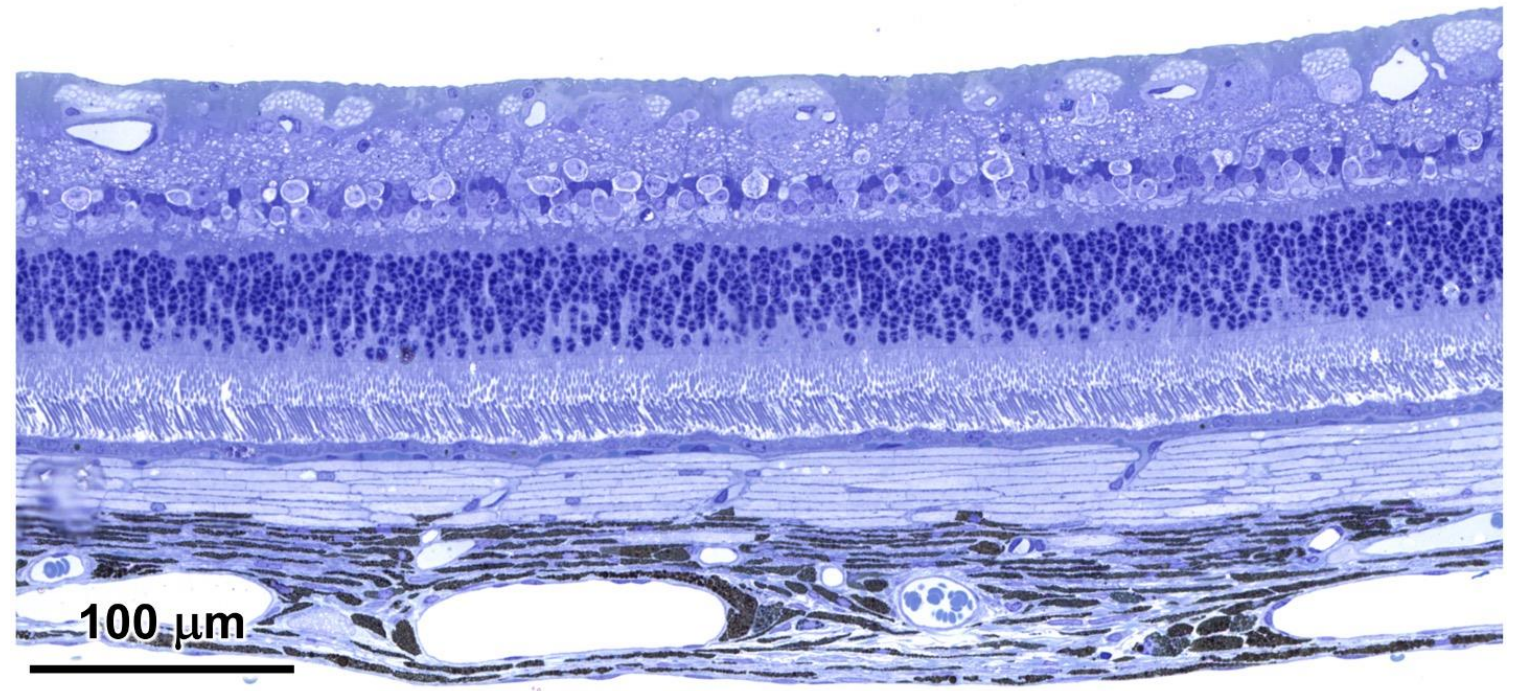

Figure 5-8: Light micrograph of a section of the superior-central retina from dog $\mathrm{Y}$, euthanized at 23.4 months of age. All layers of the retina were well preserved with no apparent morphological abnormalities.

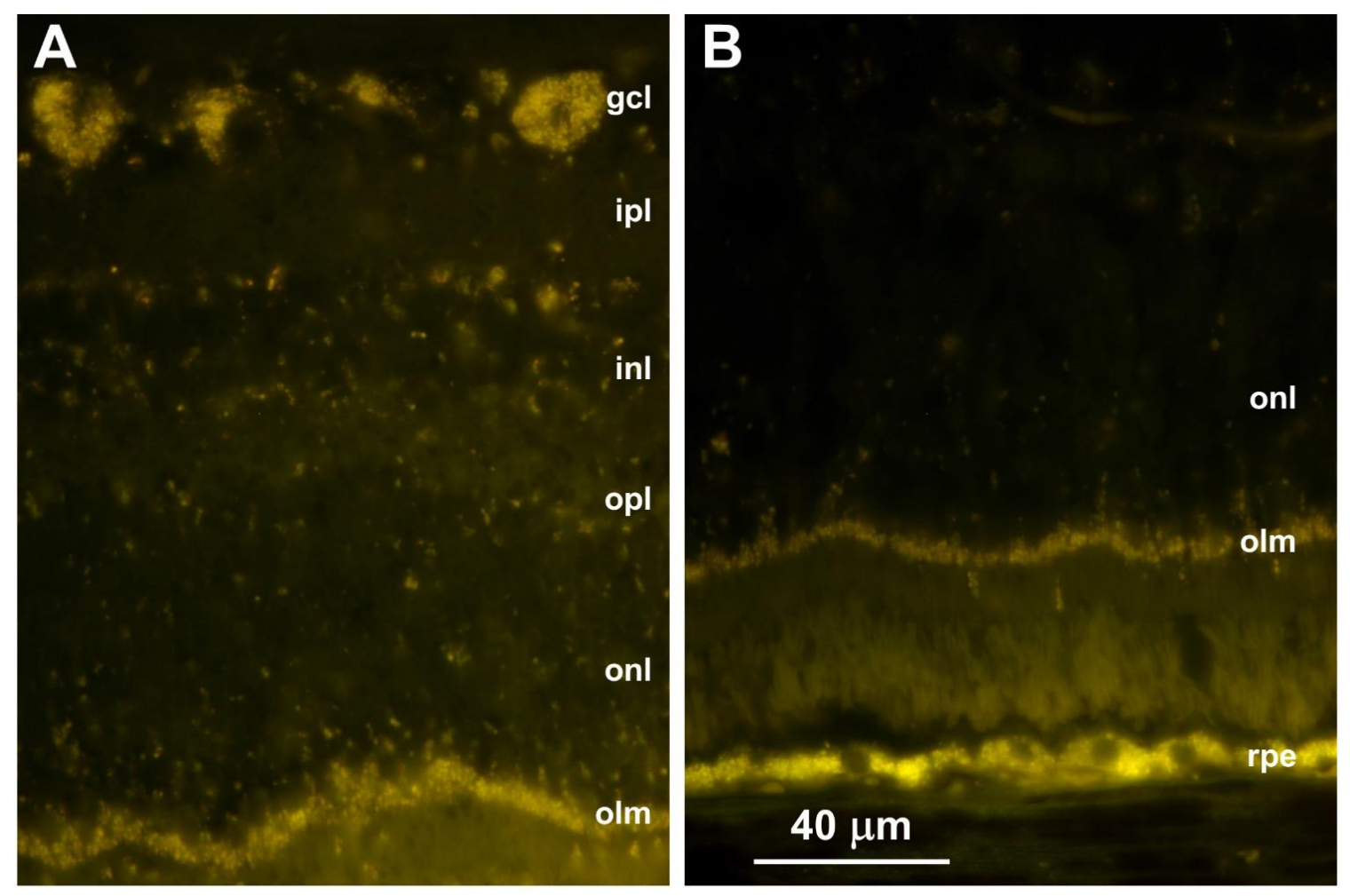

Figure 5-9: Fluorescence micrographs of the inner $(A)$ and outer $(B)$ layers of unstained cryostat sections of the retina from dog $Z$, euthanized at 23.4 months of age. Bar in (B) indicates the magnification of both micrographs. 

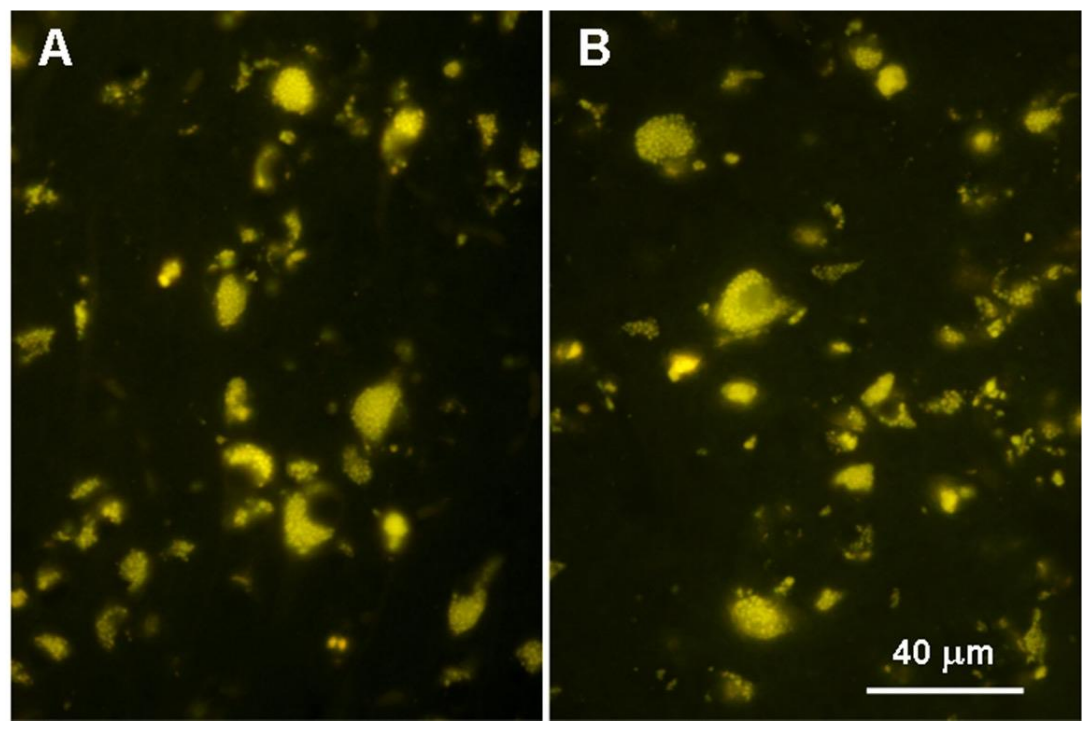

Figure 5-10: Fluorescence micrographs of unstained cryostat sections of the occipital cortex from dog $V(A)$ and $\operatorname{dog} Y(B)$, euthanized at 20.1 and 23.4 months of age respectively. Bar in (B) indicates the magnification of both micrographs.

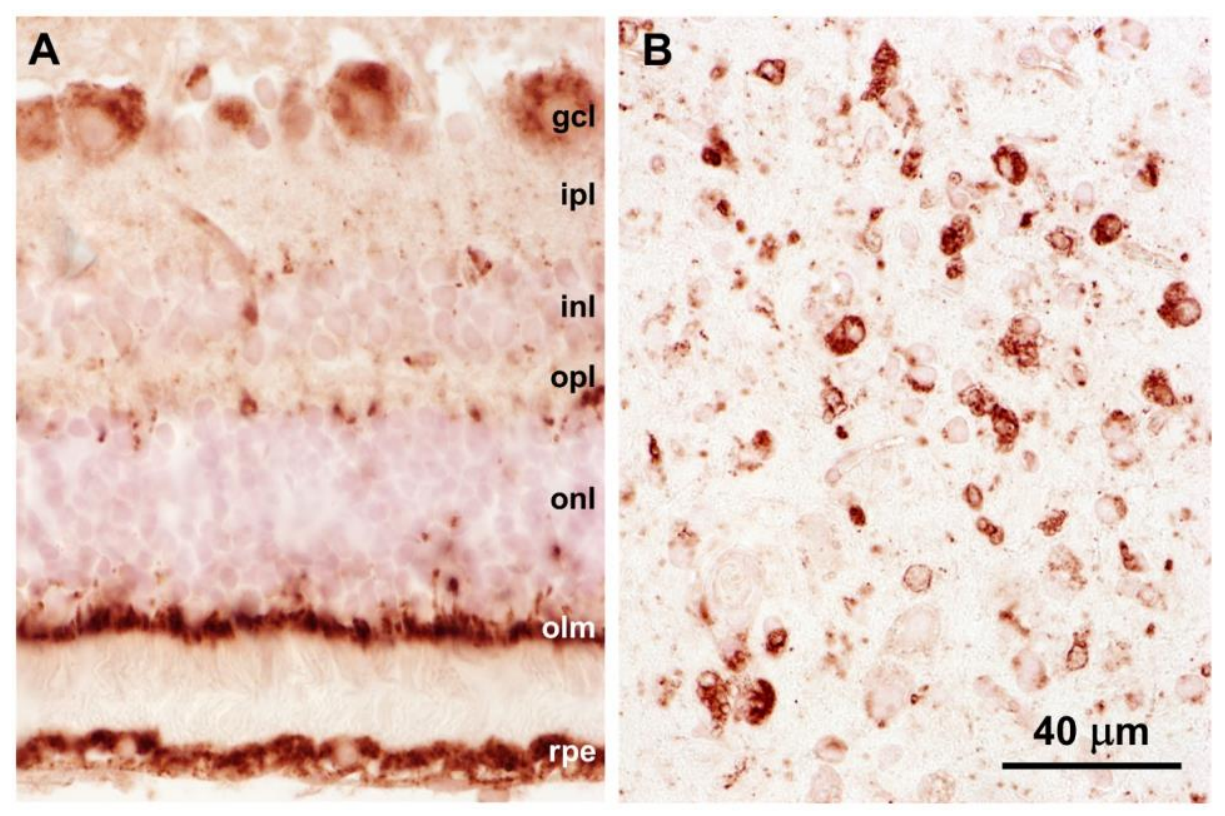

Figure 5-11: Light micrographs of sections of the retina (A) and occipital cortex (B) from dog X. Sections were immunostained for LAMP2 localization. No counterstain was used. In the retina, LAMP2 immunostaining was most pronounced in the ganglion cells, along the outer limiting membrane, and in the RPE. In the occipital cortex most cells contained aggregates of LAMP2-positive inclusions. Bar in (B) indicates the magnification of both micrographs. 
Upon electron microscopic examination, the storage bodies in the neural retina and neurons of the occipital cortex were seen to consist of membranebounded organelles with contents whose ultrastructure varied with cell type (Figures 5-12 to 5-14). Already by 20 months of age, almost every neuron in the in the occipital cortex contained the storage bodies, the contents of which consisted primarily of membrane-like structures that were vesicular or linear (Figure 5-12). In the retinal ganglion cells, in certain planes of section the storage body contents had a paracrystalline appearance (Figure 5-13). With electron microscopy, the storage bodies along the outer limiting membrane were found to be localized almost exclusively to the regions of the photoreceptor cells just interior to the junctional complexes that form the outer limiting membrane (Figure 5-14). The contents of these storage bodies consisted primarily of vesicular structures. These storage bodies appeared to be present in every photoreceptor cell. The storage bodies in the RPE were more heterogenous in appearance (Figures 5-15 and 5-16). Some of the storage bodies contained what appeared to be remnants of partially degraded photoreceptor outer segments or melanosomes (Figure 5-15). The contents of other storage bodies consisted of heterogenous mixtures of components of variable fine structure. Some of the inclusions were surrounded by double membranes characteristic of autophagosomes (Figure 5-15C). 


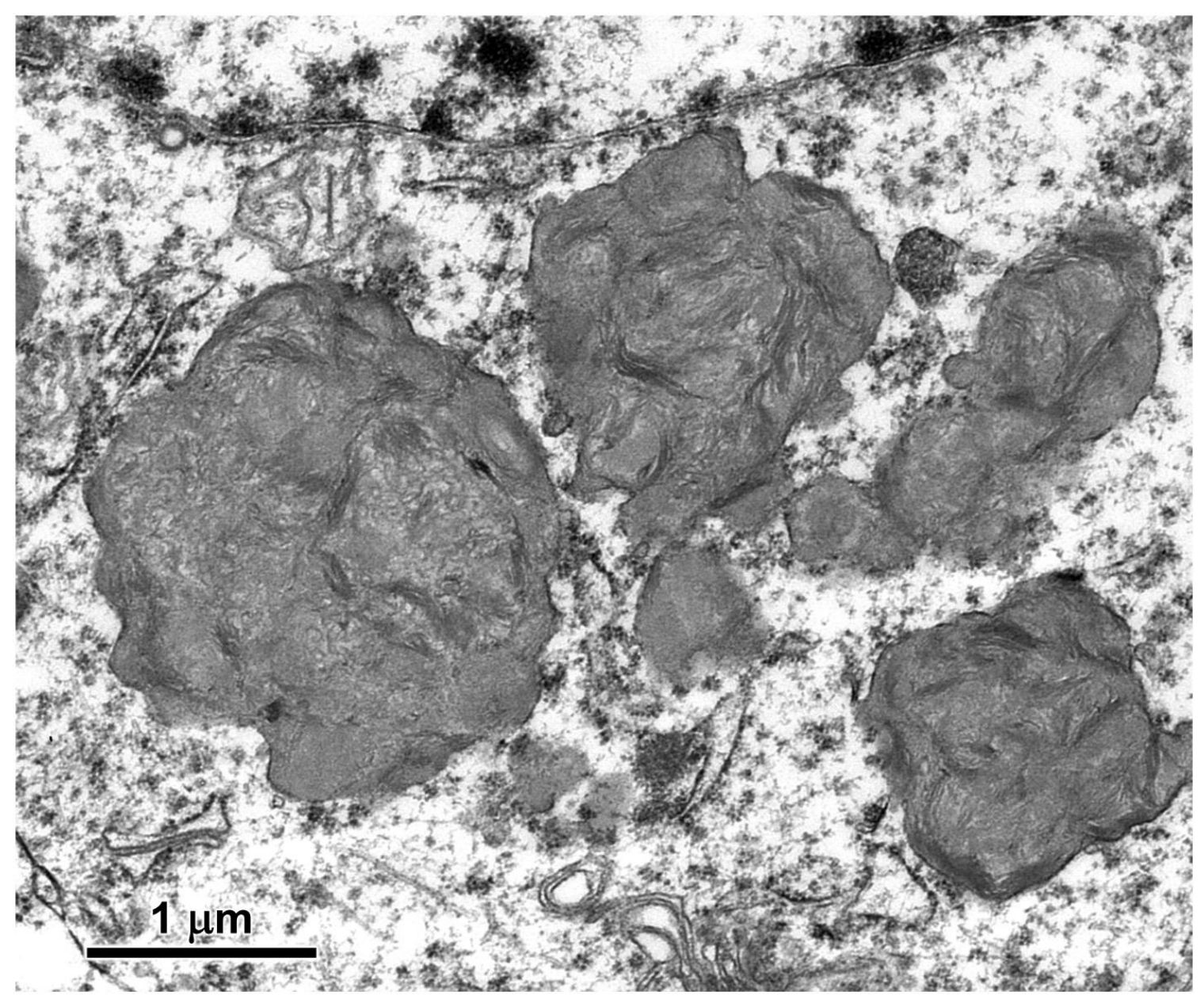

Figure 5-12: Electron micrograph of a neuron in the occipital cortex of an affected Golden Retriever (dog V) euthanized at approximately 20 months of age. 

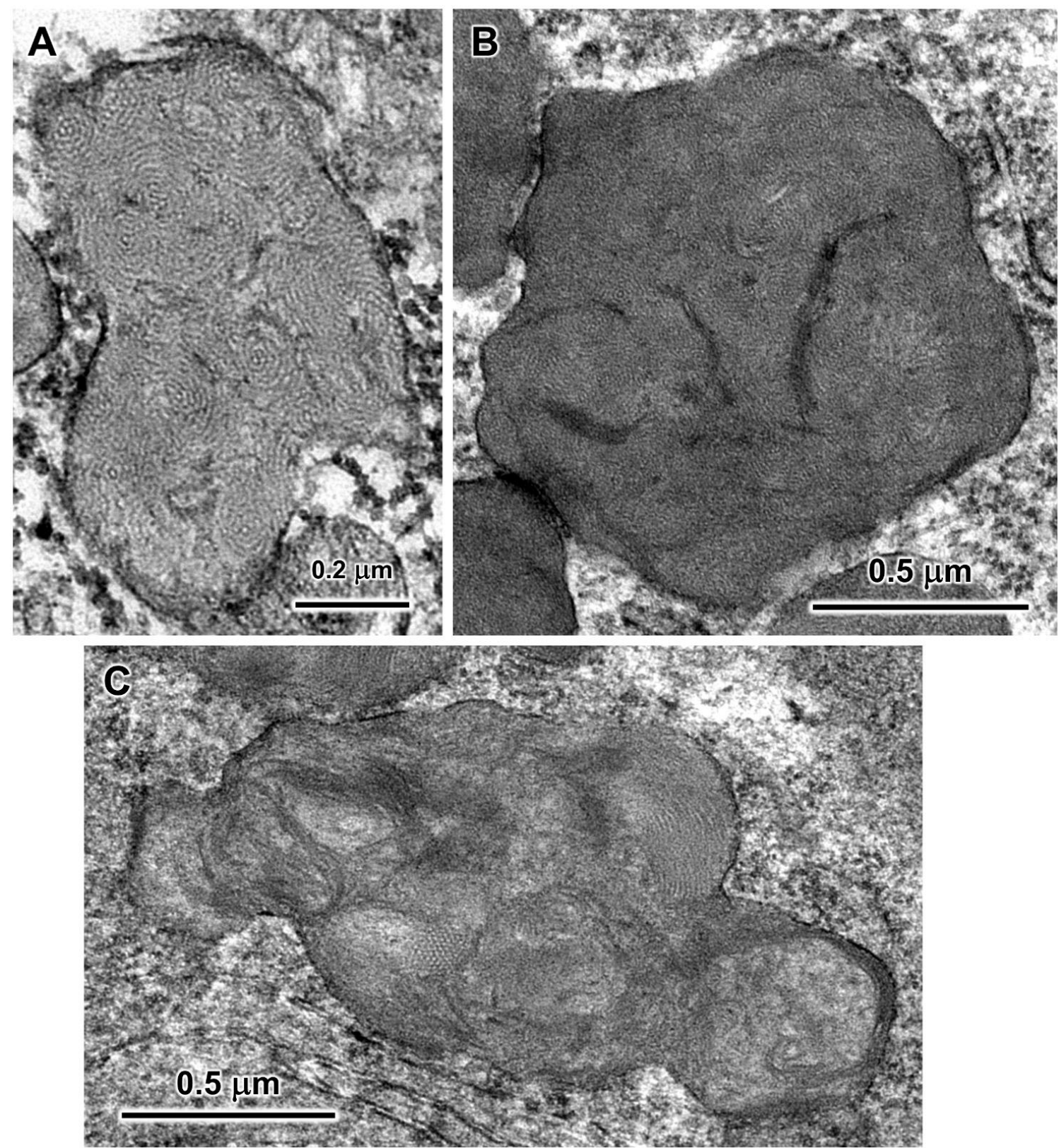

Figure 5-13: Electron micrographs of disease-specific storage bodies in retinal ganglion cells of an affected Golden Retriever (dog V) euthanized at approximately 20 months of age. In certain planes of section some of the contents of the storage bodies had a paracrystalline appearance. 


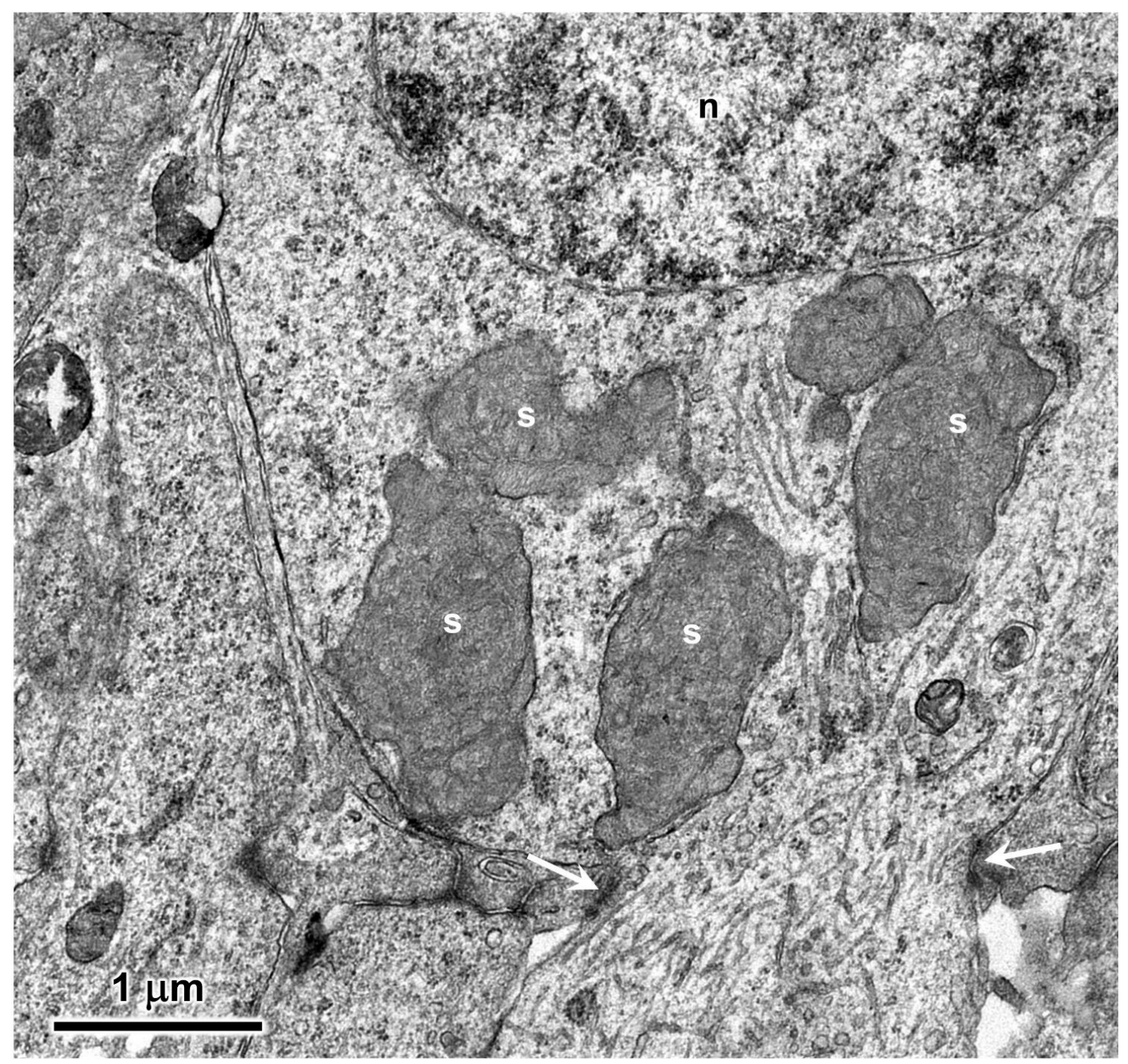

Figure 5-14: Electron micrograph showing an accumulation of disease-specific storage bodies (s) in a photoreceptor cell of an affected Golden Retriever (dog V) euthanized at approximately 20 months of age. The storage bodies were present almost exclusively just interior to the retinal outer limiting membrane (arrows) and exterior to the photoreceptor nuclei (n). 


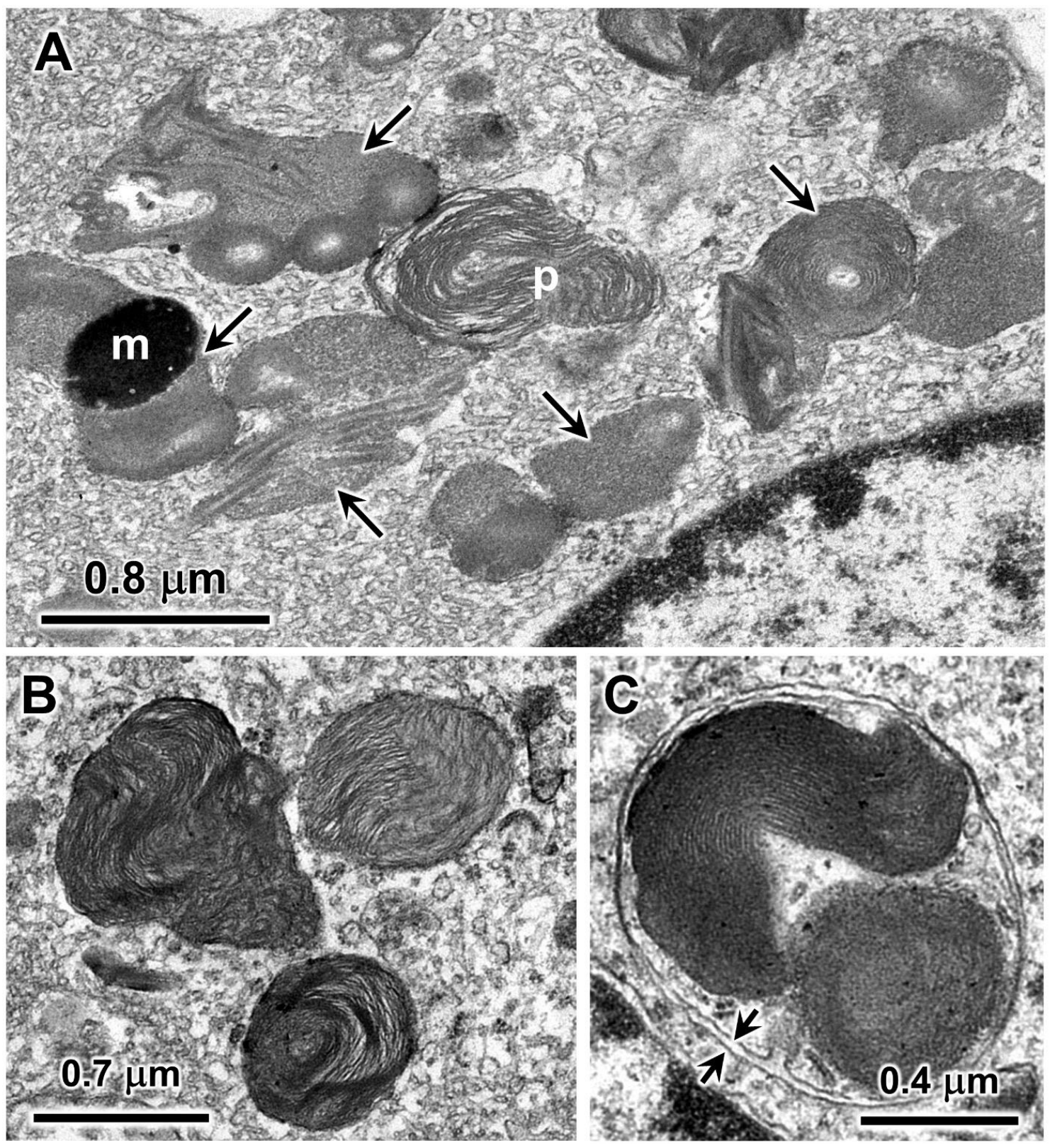

Figure 5-15: Electron micrographs of disease-specific storage bodies in the retinal pigment epithelium of an affected Golden Retriever (dog W) euthanized at approximately 22 months of age. The ultrastructural appearances of the storage bodies (arrows in A and inclusions in B and $\mathrm{C}$ ) were quite heterogenous. Some inclusions contained melanosomes $(\mathrm{m})$ and a small minority appeared to be phagocytosed photoreceptor outer segments $(p)$. The contents of some inclusions had a membrane-like appearance (B). Some inclusions were surrounded by a double membrane characteristic of autophagosomes (arrows in C). 

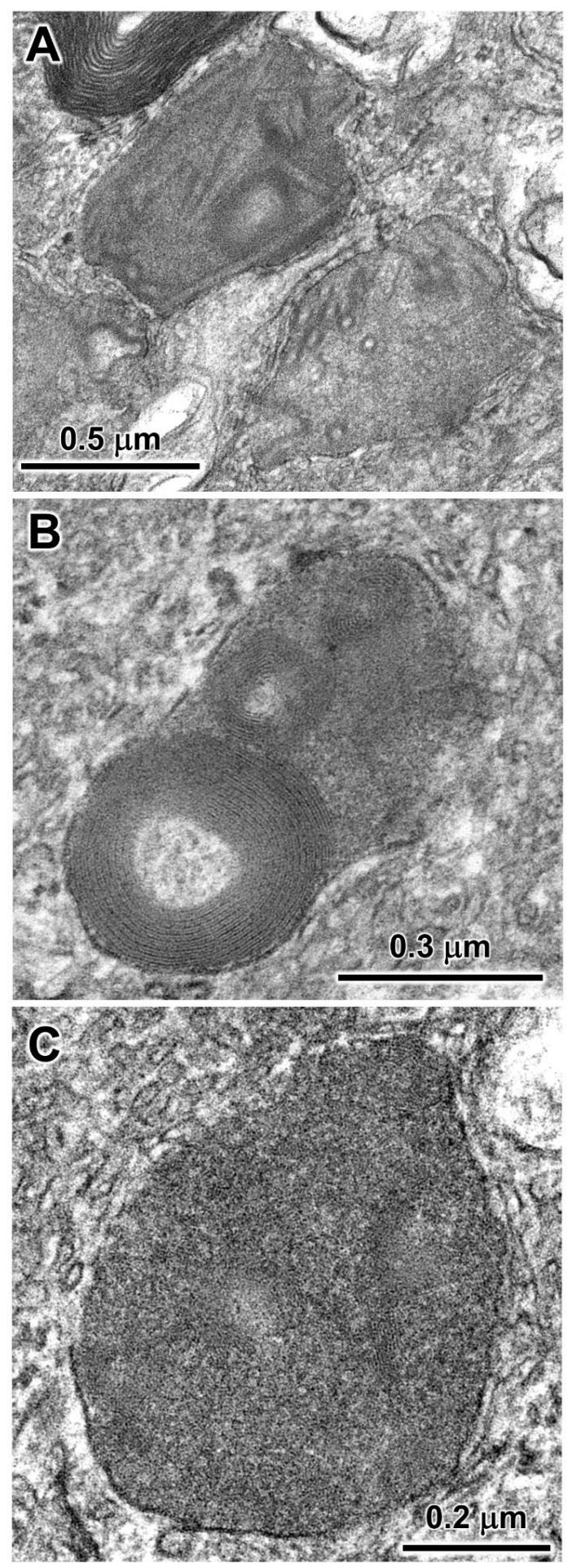

Figure 5-16: Electron micrographs of additional disease-specific storage bodies in the retinal pigment epithelium of an affected Golden Retriever (dog W) euthanized at approximately 22 months of age. The ultrastructural appearances of the storage bodies were quite heterogeneous, even within the same eye. 
Optic nerve sections were stained with Masson's trichrome which stains collagenous connective tissue components blue and most cellular components red or pink. In the normal dog retina, the axons are divided into fascicles surrounded by connective tissue trabeculae (Balaratnasingam et al., 2009; Donovan et al., 1974; COPLOW, personal communication). In the CLN5-affected dogs, these septa were greatly diminished resulting in reduced boundaries between fascicles (Figure 5-17).

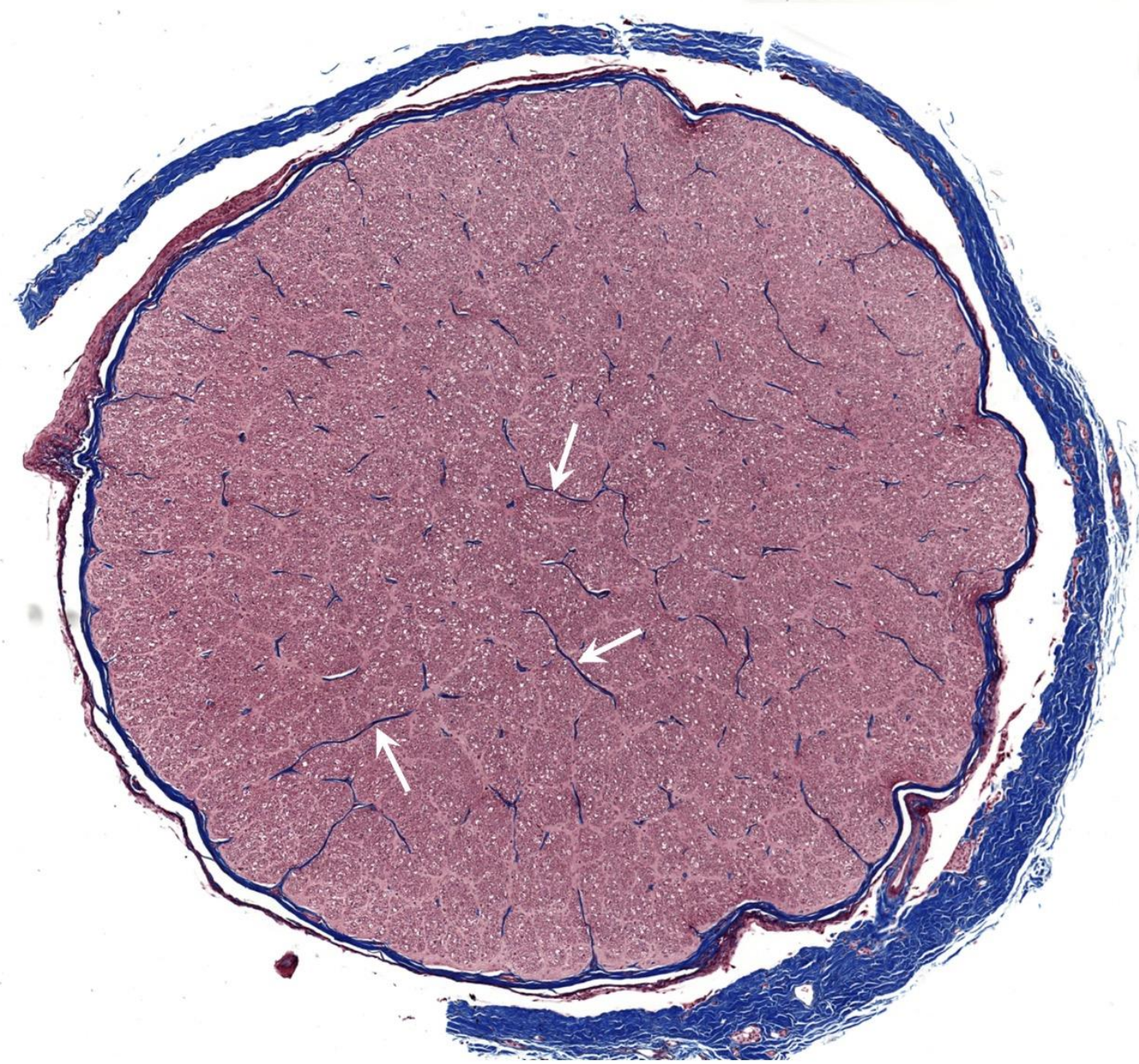

Figure 5-17: Light micrograph of a cross-section of the optic nerve from $\operatorname{dog} Y$ stained with Masson's trichrome. The blue-staining connective tissue septa (arrows) only partially surround axon fascicles. 


\subsection{Discussion}

Most children afflicted with CLN5 disease exhibit progressive vision loss starting early in the disease process (Åberg et al., 2011; Pineda-Trujillo et al., 2005; Santavuori et al., 1991, 1982). In two groups of Finnish patients, visual decline first became apparent at 5 to 8 years of age, and all children examined were blind by 9 to 10 years of age (Santavuori et al., 1991, 1982). In this group of patients, the electroretinogram (ERG) was extinguished by 8 years of age. These findings indicated that vision loss in this disorder is due at least in part to impaired retinal function. Visual evoked potentials (VEPs) were assessed in affected children to determine whether central nervous system processing of visual signals is altered in this disorder. The first change, pathologically elevated VEPs, occurred early in the preclinical stage of the disease process in some children while in others this was not obvious until 8 to 9 years of age. In some of the children the VEP responses then declined, eventually becoming nonrecordable (Santavuori et al., 1991, 1982). Similar results - abolished ERGs at 6 to 8 years of age and elevated flash VEPs at 6 to 10 years of age - were reported in a separate study which included Dutch and Swedish patients (Holmberg et al., 2000). Children from another cohort of patients from several countries exhibited decreased ERG amplitudes and abnormal VEPs (increased latency and reduced amplitude) (Simonati et al., 2017). Ophthalmoscopic examinations of children with CLN5 disease revealed generalized retinal dystrophy which progressed to optic nerve atrophy at 7 to 9 years of age with 
narrowing of retinal vessels and pigment changes (Santavuori et al., 1991, 1982). It is well documented that CLN5 disease in children is characterized by progressive global brain atrophy, including regions of the brain involved in visual signal processing (Autti et al., 1992; Tyynelä et al., 1997). Thus, the evidence from human studies indicates that vision loss in CLN5 disease results from a combination of progressive impairment of retinal and CNS function.

Dogs with naturally occurring variants in CLN5 suffer from a progressive neurodegenerative disorder very similar to many human cases of CLN5 disease (Gilliam et al., 2015; Kolicheski et al., 2016; Melville et al., 2005; Villani et al., 2019). To determine whether the visual system pathology seen in children also occurs in affected dogs, we evaluated visual and retinal function in five CLN5affected Golden Retrievers (Kick et al., 2021). Clinical observations identified clear deficits in visually mediated behavior at 21 to 22 months of age, similar to what has been seen in Border Collies and Australian cattle Dogs with CLN5 disease (Gilliam et al., 2015; Kolicheski et al., 2016; Sisk et al., 1990; Studdert and Mitten, 1991). Visual deficits worsened over time and the dogs exhibited pronounced visual deficits by 23 months of age. It is challenging to assess visual capabilities in animals. We used a cotton ball visual tracking test that has been described previously as part of neuro-ophthalmic evaluations (Gelatt et al., 2013; Skerritt, 2018). While this is a good method of assessing vision in canine subjects, CLN5-affected Golden Retrievers are similar to children with CLN5 disease in that they exhibit both visual and cognitive deficits. In addition to functional impairment of the retina, progressive cognitive decline would be 
expected to alter performance on the visual tracking test. The progressive changes in tracking ability were striking and were consistent with observations that the dogs had difficulty with visually mediated navigation at the later ages, often bumping into objects when moving about in familiar surroundings under both bright and dim light conditions. Because visually mediated behavior involves both the visual pathway and neural pathways involved in cognition, it is not possible to assess the relative contributions of these two pathways using behavioral testing.

To objectively determine whether altered retinal function may have contributed to the visual behavior deficits, ERG recordings were performed over the course of the disease progression. ERG recordings showed a progressive decline in b-wave amplitudes in the dark-adapted state. After an initial decline in photopic ERG amplitudes between 8 and 12 months of age, the response amplitudes stabilized until approximately 20 months of age, after which they declined further by 23 months of age. Early in the disease progression, the photopic b- to a-wave amplitude ratios remained constant, but between 20 and 23 months of age there was a significant decline in this ratio. This could indicate that late in the disease progression inner retinal function was affected to a greater degree than photoreceptor cell function, or alternatively, that synaptic pathology inhibited signal transmission from photoreceptor cells to the inner retina. It was not possible to follow the dogs past 23 months of age because at this age, in addition to having visual deficits, their coordination was severely impaired, they were hyperactive, and they had begun to suffer from mild 
seizures. This put the dogs at high risk for injury, making it necessary to euthanize them. Therefore, it was not possible to follow the dogs to the same degree of disease progression that affected children reach prior to succumbing to the disease.

To assess whether central nervous system components of the visual pathway are affected in the canine disorder, VEPs were recorded over the course of disease progression. The P2 component of the VEP, which reflects postsynaptic cortical responses, decreased in amplitude between 20 and 23 months of age. Because the VEPs are recorded with bright light stimuli under photopic conditions, they reflect responses from retinal cone inputs. To determine whether the decreased P2 amplitudes were due to decreased signal input to the visual cortex or to more central components of the visual pathway, the ratio of the $\mathrm{P} 2$ amplitudes to the cone b-wave amplitudes were determined for each dog at each age. This ratio actually increased over the course of the disease progression. Thus, for the same input from the retina, the cortical response reflected by the VEP P2 wave was increased. The VEP data therefore indicate that there are alterations in visual signal processing in the brain. The neural generators of the components of the VEP are not well defined, but a topographic mapping study in dogs reported that P2 reflects the conduction of signal from the retina to the lateral geniculate nucleus (Kimotsuki et al., 2005). In addition to the effects of the disease on VEP P2 amplitudes, starting at 16 months of age some of the affected dogs exhibited additional peaks in the VEP responses that are not normally present in the canine VEP. These peaks may be 
indicative of aberrant visual signal processing in the brain. This is not unexpected since, as in the human disorder, canine CLN5 disease is characterized by progressive global brain atrophy (Autti et al., 1992; Santavuori et al., 2001; Simonati et al., 2017; Tyynelä et al., 1997).

Ophthalmoscopically, the only retinal abnormalities that developed in the affected dogs were a small number of focal retinal detachments. Similar detachments, but larger in size and greater in number, develop in dogs with the CLN2 form of NCL (Whiting et al., 2015). Similar lesions have not been reported in children with either CLN5 or CLN2 disease, so these lesions may be specific to dogs. However, it is possible that these lesions occur in human subjects but have been overlooked. The development of these lesions is likely to be indicative of alterations in the important interactions between the photoreceptor cells and the apposed RPE. The optic atrophy reported in some children with late-stage CLN5 disease was not observed in the affected dogs. This may be due to the fact that the dogs were euthanized at an earlier stage in the disease progression than the stage at which optic nerve atrophy was noted in affected children.

Consistent with the ophthalmoscopic findings, no gross abnormalities were observed histologically in the retinas of the affected dogs. However, there was extensive accumulation of autofluorescent membrane-bound inclusion bodies in almost all retinal layers, as well as in the occipital cortex, by 20 months of age. The lysosomal marker protein LAMP2 was localized to the inclusions, indicating that they were lysosomal storage bodies. Although the precise function of the CLN5 protein has not been determined, the protein localized to the lysosomal 
lumen and appears to be necessary for normal lysosome function (Basak et al., 2021; Isosomppi et al., 2002; Lebrun et al., 2009; Schmiedt et al., 2010). The ultrastructure of the storage bodies varied between cell types, and sometimes within the same cell type. This suggests that CLN5 deficiency results not in a failure of lysosomal degradation of a specific biochemical substrate, but in a more general impairment of lysosomal function. Thus, the material that accumulates in the lysosomes of a specific cell type appears to be dependent on what that cell type normally degrades via the lysosomal pathway. In the retinal ganglion cells, the contents of the storage bodies had a paracrystalline appearance, suggesting the failure to degrade primarily a single substrate or a small number of substrates. In the RPE, on the other hand, the ultrastructure of the storage body contents was much more heterogenous, consistent with impaired degradation of both phagocytosed photoreceptor outer segments and RPE cellular components incorporated into lysosomes via autophagy. The contents of some of the RPE inclusions appeared to consist of partially degraded photoreceptor outer segment fragments. Because the dogs were euthanized at a time in the daily light cycle at which outer segment disc shedding is expected to be minimal, the presence of these inclusions may be indicative of impairment in degradation of this phagocytosed material (Katz and Shanker, 1989). Storage material accumulation was especially pronounced in the RPE and in the retina along the outer limiting membrane. Although RPE accumulation of storage bodies has been reported in mouse models of the CLN3 and CLN6 forms of NCL, it has not been observed in animals or humans with other NCL types, nor in 
a human subject with CLN3 disease (Bensaoula et al., 2000; von EisenhartRothe et al., 2018; Wavre-Shapton et al., 2015). The autofluorescence properties of the RPE storage material are similar to those of RPE age pigment (lipofuscin), however, the ultrastructural appearance of the storage material is distinctly different from that of lipofuscin (Boulton et al., 1990; Gouras et al., 2018; Katz et al., 1986; Katz and Robison, 1984).

The lysosomal storage material along the OLM was localized to photoreceptor cells just interior to the junctional complexes between the photoreceptor and Mueller cells. Accumulation of lysosomal storage material in photoreceptor cells has not been reported for any other form of NCL. These data indicate that the lysosomal turnover within photoreceptor cells is much more pronounced that has been previously recognized. The fact that accumulation of these storage bodies occurs in the CLN5 disease and not in other disorders affecting lysosome function indicates that the CLN5 protein plays a role that is central to normal lysosomal function in this cell type. The localization of the storage material to only the region of the photoreceptors between the outer nuclear layer and the outer limiting membrane indicates that there is specific functional compartmentalization of lysosomes to this part of the cells.

Pronounced lysosomal storage body accumulation was present in almost every neuron in the brain occipital cortex. It is not known whether the presence of this storage material is detrimental to cell function. However, the presence of this storage material strongly suggests that the CLN5 protein is normally expressed in this region of the brain involved in visual signal processing. 
Although age- and breed-matched unaffected Golden Retrievers were not available for comparison, data from previous studies clearly indicate that the changes observed in the affected dogs over the time course of observation were disease-related. While absolute amplitude values should not be directly compared amongst different breeds, studies have shown that in healthy dogs of various breeds ERG amplitudes do not significantly decline over the age span included in this study. For example, no changes in ERG response amplitudes or funduscopic appearances of the retinas were observed in healthy Dachshunds and Briard dogs monitored over similar age ranges (Narfström et al., 2005, 2003; Whiting et al., 2020a, 2020b). Likewise, in healthy dogs VEPs do not change significantly over the age span over which the affected Golden Retrievers were evaluated (Kimotsuki et al., 2005). In addition, the retinal pathology observed in the affected Golden Retrievers does not occur in healthy dogs of similar ages (Dufour et al., 2020; Narfström et al., 2005, 2003; Whiting et al., 2020a, 2020b).

In addition to dogs, naturally occurring CLN5 diseases have been identified in sheep and cattle (Frugier et al., 2008; Houweling et al., 2006; Kolicheski et al., 2016; Melville et al., 2005; Villani et al., 2019). The disorder has been extensively characterized in Borderdale sheep (Amorim et al., 2015; Frugier et al., 2008;

Perentos et al., 2016, 2015; Russell et al., 2018). Affected sheep have been bred and utilized in therapeutic intervention studies (Best et al., 2017; Hughes et al., 2014; Mitchell et al., 2018; Murray et al., 2021). Blindness is a prominent sign in CLN5-affected sheep which exhibit impaired vision starting at 10 to 12 months of age and progressing to functional blindness (bilateral absence of menace 
response and loss of visual tracking) by 13 months of age. Slow PLR and severely diminished mixed receptor ERG responses are apparent at 15 to 17 months of age. Fundoscopy in affected sheep reveals extremely hyperreflective foci and thinned retinal vasculature (Mitchell et al., 2018). The sheep model has been a very useful large animal model for this disorder. A canine model would complement the sheep model and would be more accessible for research at the larger number of institutions that have resources for maintaining research dogs. Due to the risk of injury that accompanied behavioral changes and motor deficits, the dogs evaluated in this study were euthanized somewhat early in the disease progression. While the dogs that were evaluated in this report were euthanized before 24 months of age, previously studied affected companion Golden Retrievers that were maintained in home environments were kept alive as late as 30 months of age. For the canine model to be most useful for testing the longterm efficacy of therapeutic interventions, it would be advisable to breed the disease variant into a smaller breed that can be more easily maintained in a research environment even when significantly neurologically impaired (Katz et al., 2015, 2014).

Canine models have been proven invaluable in preclinical testing and can provide strong evidence to support clinical trials. We have used a Dachshund model of CLN2 disease extensively to test therapies targeting the brain and retina (Katz et al., 2015, 2014; Tracy et al., 2016; Whiting et al., 2020b, 2020a). An intrathecal enzyme replacement therapy approach tested in this model led to the first disease-modifying therapy for an NCL and data showing efficacy of 
intravitreal enzyme replacement therapy supported an investigational new drug application (Schulz et al., 2018). Intrathecal gene therapy data from the sheep model of CLN5 disease is promising (Mitchell et al., 2018). However, to preserve visual function it will likely be necessary to target both the brain and retina since pathology in both probably contributes to vision loss in this disease. Based on previous work and the findings reported here, it appears that canine CLN5 disease will be a useful model for testing therapies to preserve functional vision. It was recently reported that intravitreal injection of an AAV9. CLN5 vector prior to the onset of visual signs in a sheep model of CLN5 disease resulted in preservation of retinal structure and function for over 15 months post-injection (Murray et al., 2021). Therapeutic intervention studies using a canine model of the CLN2 form of NCL indicate that protein replacement and ex-vivo gene therapy are also promising approaches for treating the visual system pathology in CLN5 disease (Tracy et al., 2016; Whiting et al., 2020b, 2020a). 


\section{Summary and Future Directions}

We tested three different approaches to deliver functional TPP1 protein to the retina of CLN2-affected dogs to preserve retinal function: intravitreal enzyme replacement therapy, ex vivo cell-mediated gene therapy, and in vivo intravitreal gene therapy. We showed that all three methods can preserve retinal function to some extent, while each approach has advantages and disadvantages.

Intravitreal ERT was able to fully preserve ERG b-wave amplitudes when administered pre-symptomatically and post-symptomatic treatment prevented further declines in amplitudes through 10 months of age. A major advantage of this approach is the ability to administer known amounts of TPP1 in a controlled manner and to stop treatment should any side effects be observed. However, this method requires relatively frequent lifelong intravitreal injections and the treatment resulted in significant intraocular inflammation at least in the canine model. The intravitreal ERT studies were sponsored by BioMarin Pharmaceutical and, pending FDA approval, this approach should soon enter clinical trials.

Cell mediated ex vivo gene therapy partially preserved retinal function. While the therapeutic benefit was not as pronounced as that seen with intravitreal ERT or in vivo gene therapy, treatment was well tolerated and did not result in intraocular inflammation. An additional benefit to this approach is the immunomodulatory and neurotrophic effects of MSCs which may address disease-related inflammation. This therapy may require fewer injections than the 
ERT approach; however, we also showed that repeated dosing of TPP1-MSCs is safe and well tolerated should the effects wane over time.

In contrast, repeated dosing with the in vivo gene therapy vector is unlikely to be possible due to the risk of an immune response against the viral capsid upon re-administration. However, intravitreal gene therapy preserved retinal function for at least 6 months following a single injection and data from other gene therapy trials indicates that the results are likely to persist for at least several years. As with intravitreal ERT, treatment resulted in significant ocular inflammation in the dogs which complicates development but is less likely to be a concern in children.

Unlike ERT, with ex vivo and in vivo gene therapies it is difficult to control the amount of TPP1 that is delivered which complicates the development of these therapies. In the case of in vivo gene therapy, increasing the dose can also increase the risk of vector toxicity. However, the data presented here support further development of any of these methods for use in children with CLN2 disease and the two alternative approaches to ERT offer advantages that make further development worthwhile.

We also tested an ex vivo cell-mediated gene therapy approach to deliver TPP1 to the central nervous system as an alternative to the ERT and gene therapy methods previously assessed in the canine model. Our results to date indicate that this was safe and well tolerated but we have not yet observed any therapeutic benefit. This project is ongoing, however, and future work will be focused on understanding why this method has not been successful. MSC-based 
therapies have been widely studied with hundreds clinical trials underway. However, variable results have been obtained in both pre-clinical and clinical studies due in part to variation in the cell product resulting from factors like donor heterogeneity and different ex vivo expansion protocols. Additionally, a major hurdle to the development of MSC-based therapies is the lack of knowledge about cell survival and migration after administration. As such, proof-of-concept studies in large animal models such as the dog are important not only for demonstrating safety and efficacy but also for thoroughly assessing those aspects of the treatment that cannot be studied in humans. Moreover, the canine disease model of CLN2 disease is extremely useful because disease state alters the function of MSC. Homing to sites of injury or inflammation and effects on the immune system are both dependent upon the microenvironment into which MSCs are administered. Therefore, it is necessary to study the behavior of the cells in a relevant disease model such as the CLN2-affected Dachshund. Future studies should focus on understanding the fate of the cells after injection by developing methods to locate and identify transplanted MSCs post-mortem or in vivo, such as with MRI tracking. Other future avenues could include testing alternate delivery strategies that may enhance retention, survival and localization of the cells such as multiple injections, different injection methods (i.e. intraparenchymal or intra-arterial administration), or delivery of encapsulated or scaffolded cells. Additionally, future studies will address the hypotheses that the dose of cells administered was too low to produce therapeutic levels of TPP1 in the central nervous system, that expression of TPP1 by the implanted cells 
waned after injection, and that the implanted cells migrated to locations where they were not able to deliver therapeutic levels of TPP1 to the brain. Testing these hypotheses may enable us to optimize this approach to therapy and thus to take advantage of its potential advantages over ERT or direct gene therapy.

These three approaches to treatment could be adapted to any lysosomal storage disorder due to a deficit of a soluble lysosomal enzyme, which includes several forms of NCL. Given that, we began to characterize a new canine disease model of the CLN5 form of NCL. We demonstrated that Golden Retrievers with CLN5 disease exhibit visual system pathology similar to that seen in the human disease. The dogs exhibited visual impairment, reduced ERG amplitudes, altered visual evoked potential recordings and extensive storage material accumulation in the retina and occipital cortex. This baseline data could provide a foundation for future therapeutic intervention studies treating visual system pathology in CLN5 disease. In ongoing work, we are characterizing neurological disease progression in this same model with the goal of obtaining similar baseline data which could be used to develop therapies targeting the CNS.

The lab has successfully treated neurological and retinal disease signs in the canine model of CLN2 disease with several approaches. However, we have so far only been able to follow the retinal therapies out to 10 months of age, when the dogs succumb to neurological disease. Additionally, because functional vision relies on both visual pathways in the brain and the retina, these dogs likely still become blind due to degeneration of the central nervous system. An exciting 
future direction will be testing a combined brain and eye therapy and assessing functional vision using a novel method we have developed to detect loss of visual acuity in CLN2-affected dogs. A combined therapy would also let us follow retinal therapies past 10 months of age, which will be particularly important for in vivo gene therapy. 


\section{Appendix A}

\section{Cell Culture Details for MSC-Treated Dogs}

\begin{tabular}{|c|c|c|c|c|c|c|c|c|c|c|c|}
\hline $\begin{array}{c}\text { Dog } \\
\text { ID }\end{array}$ & $\begin{array}{c}\text { BMA } \\
\#\end{array}$ & $\begin{array}{c}\text { Age at } \\
\text { BMA } \\
\text { (months) }\end{array}$ & $\begin{array}{c}\text { Age at } \\
\text { Injection } \\
\text { (months) }\end{array}$ & $\begin{array}{c}\text { Injection } \\
\text { Method/Location }\end{array}$ & Transgene & $\%$ FBS Used & $\begin{array}{c}\text { Injection } \\
\text { Volume } \\
(\mu \mathrm{L})\end{array}$ & $\begin{array}{c}\text { \# Plates } \\
\text { Harvested }\end{array}$ & $\begin{array}{c}\text { Passage } \\
\text { \# at } \\
\text { Harvest }\end{array}$ & $\begin{array}{l}\text { Amount of } \\
\text { Vector Used to } \\
\text { Transduce (vg) }\end{array}$ & $\begin{array}{l}\text { Injected } \\
\text { Cell } \\
\text { Count }\end{array}$ \\
\hline \multicolumn{12}{|c|}{$\begin{array}{l}\text { Dissociation reagents used: HyQTase or Accutase } \\
\text { FBS used: Characterized US Origin, } 10-20 \%\end{array}$} \\
\hline \multirow{2}{*}{5} & BMA 1 & 2.6 & 4.0 & $\mathrm{CM}$ & hTPP1 & $\begin{array}{l}20 \%, 10 \% \\
\quad \text { after } \\
\text { transduction }\end{array}$ & 1000 & 15 & 5 & not recorded & $5.38 \mathrm{E}+06$ \\
\hline & BMA 2 & 3.7 & 4.9 & $\mathrm{CM}$ & hTPP1 & $\begin{array}{l}20 \%, 10 \% \\
\quad \text { after } \\
\text { transduction }\end{array}$ & 1000 & 15 & 5 & approx. 9E+12 & $1.10 \mathrm{E}+07$ \\
\hline \multirow{4}{*}{1} & \multirow{3}{*}{ BMA 1} & \multirow{3}{*}{2.4} & 4.3 & $\mathrm{CM}$ & $\begin{array}{l}\text { hTPP1 + 25\% } \\
\text { hTPP1 \& GFP }\end{array}$ & $\begin{array}{l}20 \%, 10 \% \\
\quad \text { after } \\
\text { transduction }\end{array}$ & 1000 & 20 & 5 & $2.10 E+13$ & 4.10E+06 \\
\hline & & & \multirow{2}{*}{4.3} & IVT, OD & GFP & $\begin{array}{l}20 \%, 10 \% \\
\quad \text { after } \\
\text { transduction }\end{array}$ & 250 & 3 & 5 & $1.56 \mathrm{E}+12$ & $6.93 E+05$ \\
\hline & & & & IVT, OS & hTPP1 & $\begin{array}{l}20 \%, 10 \% \\
\quad \text { after } \\
\text { transduction }\end{array}$ & 250 & 3 & 5 & $1.23 \mathrm{E}+12$ & $7.44 E+05$ \\
\hline & BMA 2 & 3.4 & 5.3 & ICV & $\begin{array}{l}\text { hTPP1 + 25\% } \\
\text { hTPP1 \& GFP }\end{array}$ & $\begin{array}{c}20 \%, 10 \% \\
\quad \text { after } \\
\text { transduction }\end{array}$ & 1200 & 20 & 5 & $\begin{array}{l}\text { 1.12E+13 TPP1 } \\
7.32 \mathrm{E}+11 \mathrm{GFP}\end{array}$ & $6.49 E+06$ \\
\hline \multirow{3}{*}{2} & \multirow{3}{*}{ BMA 1} & \multirow{3}{*}{2.8} & \multirow{2}{*}{4.0} & IVT, OD & GFP & $20 \%$ & 200 & 3 & 5 & approx. $1 \mathrm{E}+12$ & $3.03 E+06$ \\
\hline & & & & IVT, OS & hTPP1 & $20 \%$ & 200 & 3 & 5 & approx. 1E+12 & $3.45 \mathrm{E}+06$ \\
\hline & & & 4.7 & ICV & $\begin{array}{l}\text { hTPP1 }+25 \% \\
\text { hTPP1 \& GFP }\end{array}$ & $10-20 \%$ & 1000 & 20 & 7 & $\begin{array}{l}1.2 \mathrm{E}+13 \mathrm{TPP} 1 \\
3.36 \mathrm{E}+11 \mathrm{GFP}\end{array}$ & $1.60 \mathrm{E}+07$ \\
\hline
\end{tabular}




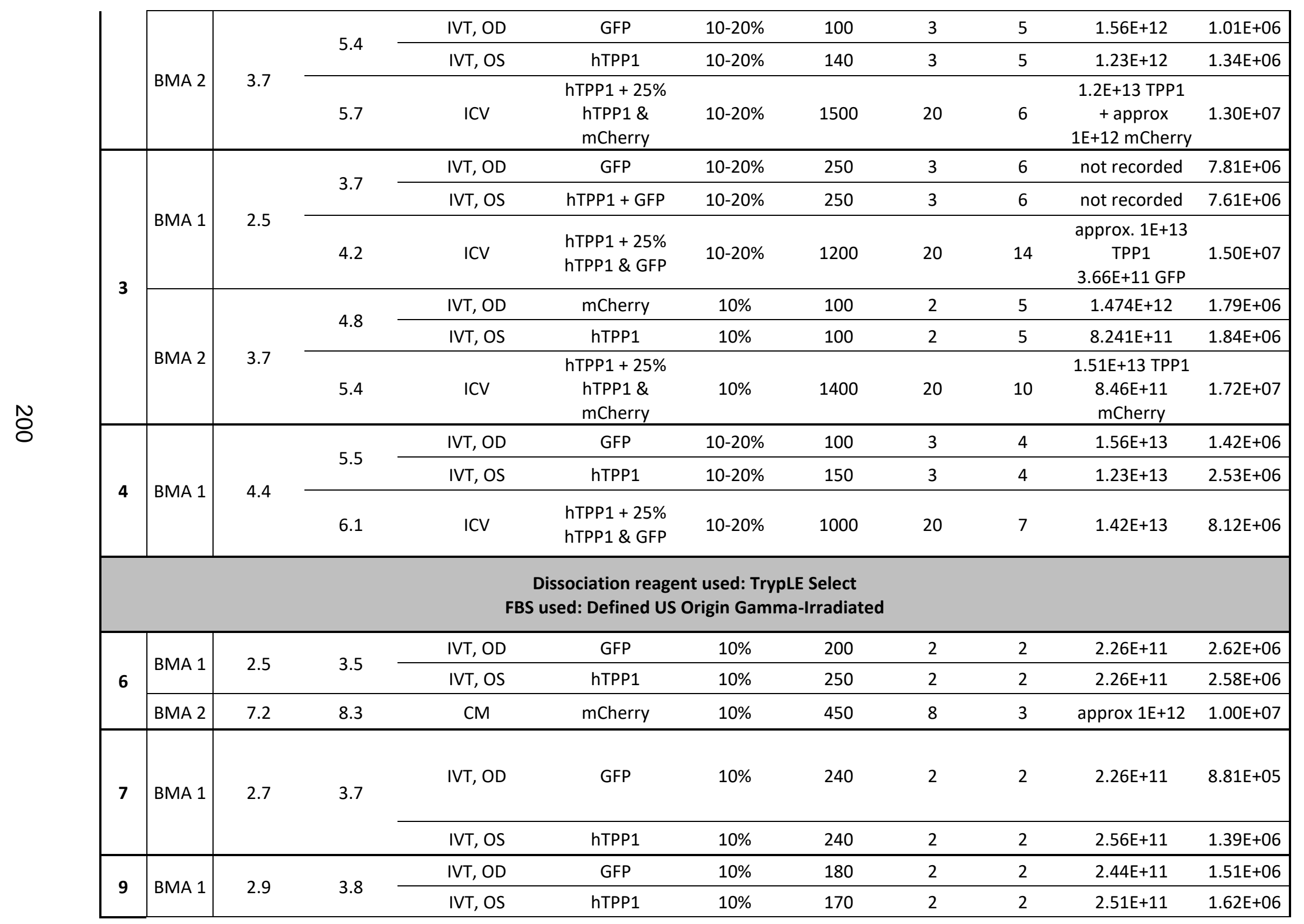




\begin{tabular}{|c|c|c|c|c|c|c|c|c|c|c|c|}
\hline & BMA 2 & 4.0 & 5.0 & $\mathrm{CM}$ & hTPP1 & $10 \%$ & 800 & 8 & 3 & $1 \mathrm{E}+12$ & $2.27 E+07$ \\
\hline 8 & BMA 1 & 4.1 & 5.0 & CM & mCherry & $10 \%$ & 0.985 & 8 & 3 & $1 E+12$ & $2.05 \mathrm{E}+07$ \\
\hline
\end{tabular}

$\mathrm{BMA}=$ bone marrow aspiration; $\mathrm{vg}=$ viral genomes; $\mathrm{CM}=$ injection at cerebromedullary cistern; $\mathrm{IVT}=$ intravitreal injection; $\mathrm{OS}$ = left eye; $\mathrm{OD}$ = right eye; ICV = intracerebroventricular injection 


\title{
Appendix B
}

\author{
Human TPP1 cDNA Sequence
}

\begin{abstract}
$5^{\prime}$
GCTAGCGGATCCGTCGACAAGCTTGGTACAAAAAAGCAGGCTCCACCATGGGACTCCAAGCCTGC CTCCTAGGGCTCTTTGCCCTCATCCTCTCTGGCAAATGCAGTTACAGCCCGGAGCCCGACCAGCG GAGGACGCTGCCCCCAGGCTGGGTGTCCCTGGGCCGTGCGGACCCTGAGGAAGAGCTGAGTCTCA CCTTTGCCCTGAGACAGCAGAATGTGGAAAGACTCTCGGAGCTGGTGCAGGCTGTGTCGGATCCC AGCTCTCCTCAATACGGAAAATACCTGACCCTAGAGAATGTGGCTGATCTGGTGAGGCCATCCCC ACTGACCCTCCACACGGTGCAAAAATGGCTCTTGGCAGCCGGAGCCCAGAAGTGCCATTCTGTGA TCACACAGGACTTTCTGACTTGCTGGCTGAGCATCCGACAAGCAGAGCTGCTGCTCCCTGGGGCT GAGTTTCATCACTATGTGGGAGGACCTACGGAAACCCATGTTGTAAGGTCCCCACATCCCTACCA GCTTCCACAGGCCTTGGCCCCCCATGTGGACTTTGTGGGGGGACTGCACCGTTTTCCCCCAACAT CATCCCTGAGGCAACGTCCTGAGCCGCAGGTGACAGGGACTGTAGGCCTGCATCTGGGGGTAACC CCCTCTGTGATCCGTAAGCGATACAACTTGACCTCACAAGACGTGGGCTCTGGCACCAGCAATAA CAGCCAAGCCTGTGCCCAGTTCCTGGAGCAGTATTTCCATGACTCAGACCTGGCTCAGTTCATGC GCCTCTTCGGTGGCAACTTTGCACATCAGGCATCAGTAGCCCGTGTGGTTGGACAACAGGGCCGG GGCCGGGCCGGGATTGAGGCCAGTCTAGATGTGCAGTACCTGATGAGTGCTGGTGCCAACATCTC CACCTGGGTCTACAGTAGCCCTGGCCGGCATGAGGGACAGGAGCCCTTCCTGCAGTGGCTCATGC TGCTCAGTAATGAGTCAGCCCTGCCACATGTGCATACTGTGAGCTATGGAGATGATGAGGACTCC CTCAGCAGCGCCTACATCCAGCGGGTCAACACTGAGCTCATGAAGGCTGCCGCTCGGGGTCTCAC CCTGCTCTTCGCCTCAGGTGACAGTGGGGCCGGGTGTTGGTCTGTCTCTGGAAGACACGAGTTCC GCCCTACCTTCCCTGCCTCCAGCCCCTATGTCACCACAGTGGGAGGCACATCCTTCCAGGAACCT TTCCTCATCACAAATGAAATTGTTGACTATATCAGTGGTGGTGGCTTCAGCAATGTGTTCCCACG GCCTTCATACCAGGAGGAAGCTGTAACGAAGTTCCTGAGCTCTAGCCCCCACCTGCCACCATCCA GTTACTTCAATGCCAGTGGCCGTGCCTACCCAGATGTGGCTGCACTTTCTGATGGCTACTGGGTG GTCAGCAACAGAGTGCCCATTCCATGGGTGTCCGGAACCTCGGCCTCTACTCCAGTGTTTGGGGG GATCCTATCCTTGATCAATGAGCACAGGATCCTTAGTGGCCGCCCCCCTCTTGGCTTTCTCAACC CAAGGCTCTACCAGCAGCATGGGGCAGGACTCTTTGATGTAACCCGTGGCTGCCATGAGTCCTGT CTGGATGAAGAGGTAGAGGGCCAGGGTTTCTGCTCTGGTCCTGGCTGGGATCCTGTAACAGGCTG GGGAACACCCAACTTCCCAGCTTTGCTGAAGACTCTACTCAACCCCTAGGACCCAGCTTTCTTGT ACCGATATCGCGGCCGCCCTAGGGAGCTCCTCGAGGCGGCCCGCTCGAGTCTAGAGGGCCCTTCG AAGGTAAGCCTATCCCTAACCCTCTCCTCGGTCTCGATTCTAACGCGTACCGGTCATCATCACCA TCACCATTGAGTTTAAAC $3^{3}$
\end{abstract}




\section{REFERENCES}

Åberg, L., Autti, T., Cooper, J.D., Elleder, M., Haltia, M., Jalanko, A., Kitzmüller, C., Kopra, O., Mole, S.E., Nuutila, A., Peltonen, L., Punkari, M.-L., Rapola, J., Tyynelä, J., 2011. CLN5, in: Mole, S., Williams, R., Goebel, H. (Eds.), The Neuronal Ceroid Lipofuscinoses (Batten Disease). Oxford University Press, Oxford, UK. https://doi.org/10.1093/med/9780199590018.003.0009

Agbandje-McKenna, M., Kleinschmidt, J., 2011. AAV Capsid Structure and Cell Interactions, in: Snyder, R.O., Moullier, P. (Eds.), Adeno-Associated Virus: Methods and Protocols. Humana Press, New York, pp. 47-92. https://doi.org/10.1007/978-1-61779-370-7_3

Amorim, I.S., Mitchell, N.L., Palmer, D.N., Sawiak, S.J., Mason, R., Wishart, T.M., Gillingwater, T.H., 2015. Molecular neuropathology of the synapse in sheep with CLN5 Batten disease. Brain Behav. 5, 1-12. https://doi.org/10.1002/brb3.401

Anderson, G.W., Goebel, H.H., Simonati, A., 2013. Human pathology in NCL. Biochim. Biophys. Acta - Mol. Basis Dis. 1832, 1807-1826. https://doi.org/10.1016/j.bbadis.2012.11.014

Archer, T.M., Boothe, D.M., Langston, V.C., Fellman, C.L., Lunsford, K. V., Mackin, A.J., 2014. Oral cyclosporine treatment in dogs: A review of the literature. J. Vet. Intern. Med. 28, 1-20. https://doi.org/10.1111/jvim.12265

Archer, T.M., Fellman, C.L., Stokes, J. V., Pinchuk, L.M., Lunsford, K. V., Pruett, S.B., Langston, V.C., Mackin, A.J., 2011. Pharmacodynamic Monitoring of Canine T-Cell Cytokine Responses to Oral Cyclosporine. J. Vet. Intern. Med. 25, 1391-1397. https://doi.org/10.1111/j.1939-1676.2011.00797.x

Autti, T., Raininko, R., Launes, J., Nuutila, A., Santavuori, P., 1992. JanskyBielschowsky variant disease: CT, MRI, and SPECT findings. Pediatr. Neurol. 8, 121-126. https://doi.org/10.1016/0887-8994(92)90032-T

Autti, T., Raininko, R., Santavuori, P., Vanhanen, S.L., Poutanen, V.P., Haltia, M., 1997. MRI of neuronal ceroid lipofuscinosis. II. Postmortem MRI and histopathological study of the brain in 16 cases of neuronal ceroid lipofuscinosis of juvenile or late infantile type. Neuroradiology 39, 371-377. https://doi.org/10.1007/s002340050427

Awano, T., Katz, M.L., O’Brien, D.P., Sohar, I., Lobel, P., Coates, J.R., Khan, S., Johnson, G.C., Giger, U., Johnson, G.S., 2006. A frame shift mutation in canine TPP1 (the ortholog of human CLN2) in a juvenile Dachshund with neuronal ceroid lipofuscinosis. Mol. Genet. Metab. 89, 254-260. https://doi.org/10.1016/j.ymgme.2006.02.016

Ba, J., Peng, R.S., Xu, D., Li, Y.H., Shi, H., Wang, Q., Yu, J., 2015. Intravitreal anti-VEGF injections for treating wet age-related macular degeneration: $A$ 
systematic review and meta-analysis. Drug Des. Devel. Ther. 9, 5397-5405. https://doi.org/10.2147/DDDT.S86269

Bainbridge, J.W.B., Mehat, M.S., Sundaram, V., Robbie, S.J., Barker, S.E., Ripamonti, C., Georgiadis, A., Mowat, F.M., Beattie, S.G., Gardner, P.J., Feathers, K.L., Luong, V.A., Yzer, S., Balaggan, K., Viswanathan, A., de Ravel, T.J.L., Casteels, I., Holder, G.E., Tyler, N., Fitzke, F.W., Weleber, R.G., Nardini, M., Moore, A.T., Thompson, D.A., Petersen-Jones, S.M., Michaelides, M., van den Born, L.I., Stockman, A., Smith, A.J., Rubin, G., Ali, R.R., 2015. Long-Term Effect of Gene Therapy on Leber's Congenital Amaurosis. N. Engl. J. Med. 372, 1887-1897.

https://doi.org/10.1056/NEJMoa1414221

Balaratnasingam, C., Morgan, W.H., Johnstone, V., Pandav, S.S., Cringle, S.J., Yu, D.-Y., 2009. Histomorphometric measurements in human and dog optic nerve and an estimation of optic nerve pressure gradients in human. Exp. Eye Res. 89, 618-628. https://doi.org/https://doi.org/10.1016/j.exer.2009.06.002

Basak, I., Wicky, H.E., McDonald, K.O., Xu, J.B., Palmer, J.E., Best, H.L., Lefrancois, S., Lee, S.Y., Schoderboeck, L., Hughes, S.M., 2021. A lysosomal enigma CLN5 and its significance in understanding neuronal ceroid lipofuscinosis. Cell. Mol. Life Sci. https://doi.org/10.1007/s00018-02103813-x

Beck-Wödl, S., Harzer, K., Sturm, M., Buchert, R., Rieß, O., Mennel, H.-D., Latta, E., Pagenstecher, A., Keber, U., 2018. Homozygous TBC1 domaincontaining kinase (TBCK) mutation causes a novel lysosomal storage disease - a new type of neuronal ceroid lipofuscinosis (CLN15)? Acta Neuropathol. Commun. 6, 145. https://doi.org/10.1186/s40478-018-0646-6

Beer, P.M., Bakri, S.J., Singh, R.J., Liu, W., Peters III, G.B., Miller, M., 2003. Intraocular concentration and pharmacokinetics of triamcinolone acetonide after a single intravitreal injection. Ophthalmology 110, 681-686. https://doi.org/10.1016/S0161-6420(02)01969-3

Beltran, W.A., Cideciyan, A. V., Guziewicz, K.E., Iwabe, S., Swider, M., Scott, E.M., Savina, S. V., Ruthel, G., Stefano, F., Zhang, L., Zorger, R., Sumaroka, A., Jacobson, S.G., Aguirre, G.D., 2014. Canine retina has a primate fovea-like bouquet of cone photoreceptors which is affected by inherited macular degenerations. PLoS One 9, 11-17. https://doi.org/10.1371/journal.pone.0090390

Bennett, J., Maguire, A.M., Cideciyan, A. V., Schnell, M., Glover, E., Anand, V., Aleman, T.S., Chirmule, N., Gupta, A.R., Huang, Y., Gao, G.P., Nyberg, W.C., Tazelaar, J., Hughes, J., Wilson, J.M., Jacobson, S.G., 1999. Stable transgene expression in rod photoreceptors after recombinant adenoassociated virus-mediated gene transfer to monkey retina. Proc. Natl. Acad. Sci. U. S. A. 96, 9920-9925. https://doi.org/10.1073/pnas.96.17.9920 
Bensaoula, T., Shibuya, H., Katz, M.L., Smith, J.E., Johnson, G.S., John, S.K., Milam, A.H., 2000. Histopathologic and immunocytochemical analysis of the retina and ocular tissues in batten disease. Ophthalmology 107, 1746-1753. https://doi.org/10.1016/S0161-6420(00)00264-5

Berns, K.I., Muzyczka, N., 2017. AAV: An Overview of Unanswered Questions. Hum. Gene Ther. 28, 308-313. https://doi.org/10.1089/hum.2017.048

Bessa, C., Teixeira, C.A.F., Mangas, M., Dias, A., Sá Miranda, M.C., Guimarães, A., Ferreira, J.C., Canas, N., Cabral, P., Ribeiro, M.G., 2006. Two novel CLN5 mutations in a Portuguese patient with vLINCL: Insights into molecular mechanisms of CLN5 deficiency. Mol. Genet. Metab. 89, 245-253. https://doi.org/10.1016/j.ymgme.2006.04.010

Best, H.L., Neverman, N.J., Wicky, H.E., Mitchell, N.L., Leitch, B., Hughes, S.M., 2017. Characterisation of early changes in ovine CLN5 and CLN6 Batten disease neural cultures for the rapid screening of therapeutics. Neurobiol. Dis. 100, 62-74. https://doi.org/10.1016/j.nbd.2017.01.001

Boucherie, C., Schäfer, S., Lavand'homme, P., Maloteaux, J.-M., Hermans, E., 2009. Chimerization of astroglial population in the lumbar spinal cord after mesenchymal stem cell transplantation prolongs survival in a rat model of amyotrophic lateral sclerosis. J. Neurosci. Res. 87, 2034-2046. https://doi.org/10.1002/jnr.22038

Boulton, M., Docchio, F., Dayhaw-Barker, P., Ramponi, R., Cubeddu, R., 1990. Age-related changes in the morphology, absorption and fluorescence of melanosomes and lipofuscin granules of the retinal pigment epithelium. Vision Res. 30, 1291-1303. https://doi.org/https://doi.org/10.1016/00426989(90)90003-4

Boyle, J., Vukicevic, M., Koklanis, K., Itsiopoulos, C., Rees, G., 2018.

Experiences of patients undergoing repeated intravitreal anti-vascular endothelial growth factor injections for neovascular age-related macular degeneration. Psychol. Health Med. 23, 127-140. https://doi.org/10.1080/13548506.2016.1274040

Braulke, T., Bonifacino, J.S., 2009. Sorting of lysosomal proteins. Biochim. Biophys. Acta - Mol. Cell Res. 1793, 605-614. https://doi.org/10.1016/j.bbamcr.2008.10.016

Breedveld, F.C., Dayer, J.M., 2000. Leflunomide: Mode of action in the treatment of rheumatoid arthritis. Ann. Rheum. Dis. 59, 841-849. https://doi.org/10.1136/ard.59.11.841

Brouwer, A.H., de Wit, G.C., ten Dam, N.H., Wijnhoven, R., van Genderen, M.M., de Boer, J.H., 2020. Electroretinogram abnormalities in non-infectious uveitis often persist. Acta Ophthalmol. 98, 627-633. https://doi.org/10.1111/aos.14401

Butz, E.S., Chandrachud, U., Mole, S.E., Cotman, S.L., 2020. Moving towards a 
new era of genomics in the neuronal ceroid lipofuscinoses. Biochim. Biophys. Acta - Mol. Basis Dis. 1866, 165571.

https://doi.org/10.1016/j.bbadis.2019.165571

Calcedo, R., Gao, G., Wilson, J.M., Vandenberghe, L.H., Lin, J., 2009. Worldwide Epidemiology of Neutralizing Antibodies to Adeno-Associated Viruses. J. Infect. Dis. 199, 381-390. https://doi.org/10.1086/595830

Caspi, R.R., 2010. A look at autoimmunity and inflammation in the eye. J. Clin. Invest. 120, 3073-3083. https://doi.org/10.1172/JCl42440

Caspi, R.R., Silver, P.B., Luger, D., Tang, J., Cortes, L.M., Pennesi, G., Mattapallil, M.J., Chan, C.C., 2008. Mouse models of experimental autoimmune uveitis. Ophthalmic Res. 40, 169-174.

https://doi.org/10.1159/000119871

Chang, M., Cooper, J.D., Davidson, B.L., van Diggelen, O.P., Elleder, M., Goebel, H.H., Golabek, A.A., Kida, E., Kohlschütter, A., Lobel, P., Mole, S.E., Schulz, A., Sleat, D.E., Warburton, M.J., Wisniewski, K., 2011. CLN2, in: Mole, S.E., Williams, R.E., Goebel, H.H. (Eds.), The Neuronal Ceroid Lipofuscinoses (Batten Disease). Oxford University Press, Oxford, pp. 80109.

Cherukuri, A., Cahan, H., de Hart, G., Van Tuyl, A., Slasor, P., Bray, L., Henshaw, J., Ajayi, T., Jacoby, D., O'Neill, C.A., Schweighardt, B., 2018. Immunogenicity to cerliponase alfa intracerebroventricular enzyme replacement therapy for CLN2 disease: Results from a Phase 1/2 study. Clin. Immunol. 197, 68-76. https://doi.org/10.1016/j.clim.2018.09.003

Choi, J.R., Yong, K.W., Wan Safwani, W.K.Z., 2017. Effect of hypoxia on human adipose-derived mesenchymal stem cells and its potential clinical applications. Cell. Mol. Life Sci. 0,1-14. https://doi.org/10.1007/s00018-0172484-2

Clément, N., Grieger, J.C., 2016. Manufacturing of recombinant adenoassociated viral vectors for clinical trials. Mol. Ther. - Methods Clin. Dev. 3, 16002. https://doi.org/10.1038/mtm.2016.2

Colombo, D., Egan, C.G., 2010. Bioavailability of Sandimmun® versus Sandimmun Neoral@: a meta-analysis of published studies. Int. J. Immunopathol. Pharmacol. 23, 1177-1183. https://doi.org/10.1177/039463201002300421

Connick, P., Kolappan, M., Patani, R., Scott, M.A., Crawley, C., He, X.L., Richardson, K., Barber, K., Webber, D.J., Wheeler-Kingshott, C.A.M., Tozer, D.J., Samson, R.S., Thomas, D.L., Du, M.Q., Luan, S.L., Michell, A.W., Altmann, D.R., Thompson, A.J., Miller, D.H., Compston, A., Chandran, S., 2011. The mesenchymal stem cells in multiple sclerosis (MSCIMS) trial protocol and baseline cohort characteristics: An open-label pre-test: Posttest study with blinded outcome assessments. Trials 12, 62. 
https://doi.org/10.1186/1745-6215-12-62

Coutinho, M.F., Prata, M.J., Alves, S., 2012. Mannose-6-phosphate pathway: A review on its role in lysosomal function and dysfunction. Mol. Genet. Metab. 105, 542-550. https://doi.org/10.1016/j.ymgme.2011.12.012

Crigler, L., Robey, R.C., Asawachaicharn, A., Gaupp, D., Phinney, D.G., 2006. Human mesenchymal stem cell subpopulations express a variety of neuroregulatory molecules and promote neuronal cell survival and neuritogenesis. Exp. Neurol. 198, 54-64. https://doi.org/10.1016/j.expneurol.2005.10.029

Cukras, C., Wiley, H.E., Jeffrey, B.G., Sen, H.N., Turriff, A., Zeng, Y., Vijayasarathy, C., Marangoni, D., Ziccardi, L., Kjellstrom, S., Park, T.K., Hiriyanna, S., Wright, J.F., Colosi, P., Wu, Z., Bush, R.A., Wei, L.L., Sieving, P.A., 2018. Retinal AAV8-RS1 Gene Therapy for X-Linked Retinoschisis: Initial Findings from a Phase I/lla Trial by Intravitreal Delivery. Mol. Ther. 26, 2282-2294. https://doi.org/10.1016/j.ymthe.2018.05.025

Dalkara, D., Byrne, L.C., Klimczak, R.R., Visel, M., Yin, L., Merigan, W.H., Flannery, J.G., Schaffer, D. V, 2013. In vivo-directed evolution of a new adeno-associated virus for therapeutic outer retinal gene delivery from the vitreous. Sci. Transl. Med. 5, 189ra76.

https://doi.org/10.1126/scitransImed.3005708

Dalkara, D., Kolstad, K.D., Caporale, N., Visel, M., Klimczak, R.R., Schaffer, D. V., Flannery, J.G., 2009. Inner Limiting Membrane Barriers to AAV-mediated Retinal Transduction From the Vitreous. Mol. Ther. 17, 2096-2102. https://doi.org/10.1038/mt.2009.181

De Silva, B., Adams, J., Lee, S.Y., 2015. Proteolytic processing of the neuronal ceroid lipofuscinosis related lysosomal protein CLN5. Exp. Cell Res. 338, 45-53. https://doi.org/10.1016/j.yexcr.2015.08.021

del Amo, E.M., Rimpelä, A.K., Heikkinen, E., Kari, O.K., Ramsay, E., Lajunen, T., Schmitt, M., Pelkonen, L., Bhattacharya, M., Richardson, D., Subrizi, A., Turunen, T., Reinisalo, M., Itkonen, J., Toropainen, E., Casteleijn, M., Kidron, H., Antopolsky, M., Vellonen, K.S., Ruponen, M., Urtti, A., 2017. Pharmacokinetic aspects of retinal drug delivery. Prog. Retin. Eye Res. 57, 134-185. https://doi.org/10.1016/j.preteyeres.2016.12.001

Dewey, C.W., Costa, R.C., Thomas, W.B., 2015. Performing the Neurologic Examination Tools for performing the neurologic examination, in: Dewey, C.W., Costa, R.C. (Eds.), Practical Guide to Canine and Feline Neurology. Wiley-Blackwell, Hoboken, pp. 9-28.

Díez-Tejedor, E., Gutiérrez-Fernández, M., Martínez-Sánchez, P., RodríguezFrutos, B., Ruiz-Ares, G., Lara, M.L., Gimeno, B.F., 2014. Reparative therapy for acute ischemic stroke with allogeneic mesenchymal stem cells from adipose tissue: A safety assessment: $A$ phase II randomized, doubleblind, placebo-controlled, single-center, pilot clinical trial. J. Stroke 
Cerebrovasc. Dis. 23, 2694-2700.

https://doi.org/10.1016/j.jstrokecerebrovasdis.2014.06.011

Dimopoulos, I.S., Hoang, S.C., Radziwon, A., Binczyk, N.M., Seabra, M.C., MacLaren, R.E., Somani, R., Tennant, M.T.S., MacDonald, I.M., 2018. TwoYear Results After AAV2-Mediated Gene Therapy for Choroideremia: The Alberta Experience. Am. J. Ophthalmol. 193, 130-142. https://doi.org/10.1016/j.ajo.2018.06.011

Ding, Subbiah, Khan, Farhana, Mok, 2019. Empowering Mesenchymal Stem Cells for Ocular Degenerative Disorders. Int. J. Mol. Sci. 20, 1784. https://doi.org/10.3390/ijms20071784

Dominici, M., Le Blanc, K., Mueller, I., Slaper-Cortenbach, I., Marini, F., Krause, D., Deans, R., Keating, A., Prockop, D., Horwitz, E., 2006. Minimal criteria for defining multipotent mesenchymal stromal cells. The International Society for Cellular Therapy position statement. Cytotherapy 8, 315-7. https://doi.org/10.1080/14653240600855905

Donovan, R.H., Carpenter, R.L., Schepens, C.L., Tolentino, F.I., 1974. Histology of the normal collie eye. III. Lens, retina and optic nerve. Ann. Ophthalmol. 6, 1299-1307.

Drack, A. V, Bennett, J., Russell, S., High, K.A., Yu, Z., Tillman, A., Chung, D., Reape, K.Z., Ciulla, T., Maguire, A., 2019. How long does gene therapy last? 4-year follow-up of phase 3 voretigene neparvovec trial in $<$ em>RPE65</em>-associated LCA/inherited retinal disease. J. Am. Assoc. Pediatr. Ophthalmol. Strabismus \{JAAPOS\} 23, e7. https://doi.org/10.1016/j.jaapos.2019.08.018

Dufour, V.L., Yu, Y., Pan, W., Ying, G.-S., Aguirre, G.D., Beltran, W.A., 2020. Invivo longitudinal changes in thickness of the postnatal canine retina. Exp. Eye Res. 192, 107926. https://doi.org/10.1016/j.exer.2020.107926

Dunn, D.W., 1987. CT in ceroid lipofuscinosis. Neurology 37, 1025-1026.

Ekesten, B., Komáromy, A.M., Ofri, R., Petersen-Jones, S.M., Narfström, K., 2013. Guidelines for clinical electroretinography in the dog: 2012 update. Doc. Ophthalmol. 127, 79-87. https://doi.org/10.1007/s10633-013-9388-8

Eleuteri, S., Fierabracci, A., 2019. Insights into the secretome of mesenchymal stem cells and its potential applications. Int. J. Mol. Sci. 20. https://doi.org/10.3390/ijms20184597

Erles, K., Sebokova, P., Schlehofer, J.R., 1999. Update on the prevalence of serum antibodies (IgG and $\operatorname{lgM}$ ) to adeno-associated virus (AAV). J. Med. Virol. 59, 406-411. https://doi.org/10.1002/(SICl)10969071(199911)59:3<406::AID-JMV22>3.0.CO;2-N

Ezaki, J., Takeda-Ezaki, M., Oda, K., Kominami, E., 2000. Characterization of endopeptidase activity of tripeptidyl peptidase-I/CLN2 protein which is 
deficient in classical late infantile neuronal ceroid lipofuscinosis. Biochem. Biophys. Res. Commun. 268, 904-8. https://doi.org/10679303

Ezaki, J., Tanida, I., Kanehagi, N., Kominami, E., 1999. A lysosomal proteinase, the late infantile neuronal ceroid lipofuscinosis gene (CLN2) product, is essential for degradation of a hydrophobic protein, the subunit $c$ of ATP synthase. J. Neurochem. 72, 2573-2582. https://doi.org/10.1046/j.14714159.1999.0722573.x

Falavarjani, K.G., Nguyen, Q.D., 2013. Adverse events and complications associated with intravitreal injection of anti-VEGF agents: A review of literature. Eye 27, 787-794. https://doi.org/10.1038/eye.2013.107

Forostyak, S., Homola, A., Turnovcova, K., Svitil, P., Jendelova, P., Sykova, E., 2014. Intrathecal Delivery of Mesenchymal Stromal Cells Protects the Structure of Altered Perineuronal Nets in SOD1 Rats and Amends the Course of ALS. Stem Cells 32, 3163-3172. https://doi.org/10.1002/stem.1812

Frugier, T., Mitchell, N.L., Tammen, I., Houweling, P.J., Arthur, D.G., Kay, G.W., van Diggelen, O.P., Jolly, R.D., Palmer, D.N., 2008. A new large animal model of CLN5 neuronal ceroid lipofuscinosis in Borderdale sheep is caused by a nucleotide substitution at a consensus splice site $(c .571+1 \mathrm{G}>\mathrm{A})$ leading to excision of exon 3. Neurobiol. Dis. 29, 306-315. https://doi.org/10.1016/j.nbd.2007.09.006

Gao, F., Chiu, S.M., Motan, D.A.L., Zhang, Z., Chen, L., Ji, H., Tse, H., Fu, Q., Lian, Q., 2016. Mesenchymal stem cells and immunomodulation : current status and future prospects. https://doi.org/10.1038/cddis.2015.327

Gardner, E., Bailey, M., Schulz, A., Aristorena, M., Miller, N., Mole, S.E., 2019. Mutation update: Review of TPP1 gene variants associated with neuronal ceroid lipofuscinosis CLN2 disease. Hum. Mutat. humu.23860.

https://doi.org/10.1002/humu.23860

Ge, L., Li, H.Y., Hai, Y., Min, L., Xing, L., Min, J., Shu, H.X., Mei, O.Y., Hua, L., 2018. Novel Mutations in CLN5 of Chinese Patients With Neuronal Ceroid Lipofuscinosis. J. Child Neurol. 33, 837-850. https://doi.org/10.1177/0883073818789024

Gelatt, K.N., Gilger, B.C., Kern, T.J. (Eds.), 2013. Veterinary Ophthalmology, 5th Ed. ed. John Wiley \& Sons, Inc., Ames, IA.

Gilliam, D., Kolicheski, A., Johnson, G.S., Mhlanga-Mutangadura, T., Taylor, J.F., Schnabel, R.D., Katz, M.L., 2015. Golden Retriever dogs with neuronal ceroid lipofuscinosis have a two-base-pair deletion and frameshift in CLN5. Mol. Genet. Metab. 115, 101-109. https://doi.org/10.1016/j.ymgme.2015.04.001

Giunti, D., Parodi, B., Usai, C., Vergani, L., Casazza, S., Bruzzone, S., Mancardi, G., Uccelli, A., 2012. Mesenchymal stem cells shape microglia effector 
functions through the release of CX3CL1. Stem Cells 30, 2044-2053. https://doi.org/10.1002/stem.1174

Goebel, H.H., Zeman, W., Damaske, E., 1977. An Ultrastructural Study of The Retina in the Jansky-Bielschowsky Type of Neuronal Ceroid- Lipofuscinosis. Am. J. Ophthalmol. 83, 70-79.

Golabek, A.A., Kida, E., Walus, M., Wujek, P., Mehta, P., Wisniewski, K.E., 2003. Biosynthesis, glycosylation, and enzymatic processing in vivo of human tripeptidyl-peptidase I. J. Biol. Chem. 278, 7135-7145. https://doi.org/10.1074/jbc.M211872200

Gouras, P., Brown, K.R., Mattison, J.A., Neuringer, M., Nagasaki, T., Ivert, L., 2018. The Ultrastructure, Spatial Distribution, and Osmium Tetroxide Binding of Lipofuscin and Melanosomes in Aging Monkey Retinal Epithelium. Curr. Eye Res. 43, 1019-1023. https://doi.org/10.1080/02713683.2018.1464194

Gowing, G., Svendsen, S., Svendsen, C.N., 2017. Chapter 4 - Ex vivo gene therapy for the treatment of neurological disorders, in: Dunnett, S.B., Björklund, A.B.T.-P. in B.R. (Eds.), Functional Neural Transplantation IV. Elsevier, pp. 99-132. https://doi.org/https://doi.org/10.1016/bs.pbr.2016.11.003

Gray, J.T., Zolotukhin, S., 2011. Design and Construction of Functional AAV Vectors, in: Snyder, R.O., Moullier, P. (Eds.), Adeno-Associated Virus: Methods and Protocols. Humana Press, New York, pp. 25-46. https://doi.org/10.1007/978-1-61779-370-7_2

Grayson, W.L., Zhao, F., Bunnell, B., Ma, T., 2007. Hypoxia enhances proliferation and tissue formation of human mesenchymal stem cells. Biochem. Biophys. Res. Commun. 358, 948-953. https://doi.org/10.1016/j.bbrc.2007.05.054

Gregori, N.Z., Davis, J.L., 2020. Surgical Observations from the First 120 Cases of Subretinal Gene Therapy for Inherited Retinal Degenerations. Retina Prepub. https://doi.org/10.1097//AE.0000000000003085

Gregory-Evans, K., M.A. Emran Bashar, A., Tan, M., 2012. Ex Vivo Gene Therapy and Vision. Curr. Gene Ther. 12, 103-115. https://doi.org/10.2174/156652312800099607

Gu, X., Yu, X., Zhao, C., Duan, P., Zhao, T., Liu, Y., Li, S., Yang, Z., Li, Y., Qian, C., Yin, Z., Wang, Y., 2018. Efficacy and Safety of Autologous Bone Marrow Mesenchymal Stem Cell Transplantation in Patients with Diabetic Retinopathy. Cell. Physiol. Biochem. 49, 40-52. https://doi.org/10.1159/000492838

Gurda, B.L., Bradbury, A.M., Vite, C.H., 2017. Canine and feline models of human genetic diseases and their contributions to advancing clinical therapies. Yale J. Biol. Med. 90, 417-431. 
Gurda, B.L., Vite, C.H., 2019. Large animal models contribute to the development of therapies for central and peripheral nervous system dysfunction in patients with lysosomal storage diseases. Hum. Mol. Genet. 00, 1-13. https://doi.org/10.1093/hmg/ddz127

Habisch, H.-J., Janowski, M., Binder, D., Kuzma-Kozakiewicz, M., Widmann, A., Habich, A., Schwalenstöcker, B., Hermann, A., Brenner, R., Lukomska, B., Domanska-Janik, K., Ludolph, A.C., Storch, A., 2007. Intrathecal application of neuroectodermally converted stem cells into a mouse model of ALS: limited intraparenchymal migration and survival narrows therapeutic effects. J. Neural Transm. 114, 1395-1406. https://doi.org/10.1007/s00702-0070748-y

Haltia, M., Goebel, H.H., 2013. The neuronal ceroid-lipofuscinoses: A historical introduction. Biochim. Biophys. Acta - Mol. Basis Dis. 1832, 1795-1800. https://doi.org/10.1016/j.bbadis.2012.08.012

Haltia, M., Herva, R., Suopanki, J., Baumann, M., Tyynelä, J., 2001. Hippocampal lesions in the neuronal ceroid lipofuscinoses. Eur. J. Paediatr. Neurol. 5, 209-211. https://doi.org/10.1053/eipn.2000.0464

Heier, J.S., Kherani, S., Desai, S., Dugel, P., Kaushal, S., Cheng, S.H., Delacono, C., Purvis, A., Richards, S., Le-Halpere, A., Connelly, J., Wadsworth, S.C., Varona, R., Buggage, R., Scaria, A., Campochiaro, P.A., 2017. Intravitreous injection of AAV2-sFLT01 in patients with advanced neovascular age-related macular degeneration: a phase 1, open-label trial. Lancet 390, 50-61. https://doi.org/10.1016/S0140-6736(17)30979-0

Heile, A., Brinker, T., 2011. Clinical translation of stem cell therapy in traumatic brain injury: the potential of encapsulated mesenchymal cell biodelivery of glucagon-like peptide-1. Dialogues Clin. Neurosci. 13, 279-286. https://doi.org/10.31887/DCNS.2011.13.2/aheile

Heile, A.M.B., Wallrapp, C., Klinge, P.M., Samii, A., Kassem, M., Silverberg, G., Brinker, T., 2009. Cerebral transplantation of encapsulated mesenchymal stem cells improves cellular pathology after experimental traumatic brain injury. Neurosci. Lett. 463, 176-181. https://doi.org/10.1016/j.neulet.2009.07.071

Holmberg, V., Lauronen, L., Autti, T., Santavuori, P., Savukoski, M., Uvebrant, P., Hofman, I., Peltonen, L., Järvelä, I., 2000. Phenotype-genotype correlation in eight patients with Finnish variant late infantile NCL (CLN5). Neurology 55, 579-581. https://doi.org/10.1212/WNL.55.4.579

Horneber, M., Gottanka, J., H. Milam, A., Lütjen-Drecoll, E., 1996. Alterations in anterior segment dimensions in eyes with retinitis pigmentosa. Graefe's Arch. Clin. Exp. Ophthalmol. 234, 71-78. https://doi.org/10.1007/BF00695244

Houweling, P.J., Cavanagh, J.A.L., Palmer, D.N., Frugier, T., Mitchell, N.L., 
Windsor, P.A., Raadsma, H.W., Tammen, I., 2006. Neuronal ceroid lipofuscinosis in Devon cattle is caused by a single base duplication (c.662dupG) in the bovine CLN5 gene. Biochim. Biophys. Acta - Mol. Basis Dis. 1762, 890-897. https://doi.org/10.1016/j.bbadis.2006.07.008

Hsuan, Y.C.Y., Lin, C.H., Chang, C.P., Lin, M.T., 2016. Mesenchymal stem cellbased treatments for stroke, neural trauma, and heat stroke. Brain Behav. 6 , 1-11. https://doi.org/10.1002/brb3.526

Hughes, S.M., Hope, K.M., Xu, J.B., Mitchell, N.L., Palmer, D.N., 2014. Inhibition of storage pathology in prenatal CLN5-deficient sheep neural cultures by lentiviral gene therapy. Neurobiol. Dis. 62, 543-550. https://doi.org/10.1016/j.nbd.2013.11.011

Isosomppi, J., Vesa, J., Jalanko, A., Peltonen, L., 2002. Lysosomal localization of the neuronal ceroid lipofuscinosis CLN5 protein. Hum. Mol. Genet. 11, 885891. https://doi.org/10.1093/hmg/11.8.885

Jules, F., Sauvageau, E., Dumaresq-Doiron, K., Mazzaferri, J., Haug-Kröper, M., Fluhrer, R., Costantino, S., Lefrancois, S., 2017. CLN5 is cleaved by members of the SPP/SPPL family to produce a mature soluble protein. Exp. Cell Res. 357, 40-50. https://doi.org/10.1016/j.yexcr.2017.04.024

Karussis, D., Petrou, P., Slavin, S., Karageorgiou, C., Gowda-Kurkalli, B., Abramsky, O., Ben-Hur, T., Gomori, J.M., Bulte, J.W.M., Kassis, I., VakninDembinsky, A., 2010. Safety and Immunological Effects of Mesenchymal Stem Cell Transplantation in Patients With Multiple Sclerosis and Amyotrophic Lateral Sclerosis. Arch. Neurol. 67, 1187-1194. https://doi.org/10.1001/archneurol.2010.248

Katz, M.L., Coates, J.R., Cooper, J.J., O'Brien, D.P., Jeong, M., Narfström, K., 2008. Retinal Pathology in a Canine Model of Late Infantile Neuronal Ceroid Lipofuscinosis. Investig. Opthalmology Vis. Sci. 49, 2686.

https://doi.org/10.1167/iovs.08-1712

Katz, M.L., Coates, J.R., Sibigtroth, C.M., Taylor, J.D., Carpentier, M., Young, W.M., Wininger, F.A., Kennedy, D., Vuillemenot, B.R., O'Neill, C.A., 2014. Enzyme replacement therapy attenuates disease progression in a canine model of late-infantile neuronal ceroid lipofuscinosis (CLN2 Disease). J. Neurosci. Res. 92, 1591-1598. https://doi.org/10.1002/jnr.23423

Katz, M.L., Drea, C.M., Eldred, G.E., Hess, H.H., Robison, W.G., 1986. Influence of early photoreceptor degeneration on lipofuscin in the retinal pigment epithelium. Exp. Eye Res. 43, 561-573. https://doi.org/https://doi.org/10.1016/S0014-4835(86)80023-9

Katz, M.L., Johnson, G.C., Leach, S.B., Williamson, B.G., Coates, J.R., Whiting, R.E.H., Vansteenkiste, D.P., Whitney, M.S., 2017a. Extraneuronal pathology in a canine model of CLN2 neuronal ceroid lipofuscinosis after intracerebroventricular gene therapy that delays neurological disease 
progression. Gene Ther. 24, 215-223. https://doi.org/10.1038/gt.2017.4

Katz, M.L., Redmond, M.T., 2001. Effect of Rpe65 knockout on accumulation of lipofuscin fluorophores in the retinal pigment epithelium. Investig. Ophthalmol. Vis. Sci. 42, 3023-3030.

Katz, M.L., Robison, W.G., 2002. What is lipofuscin? Defining characteristics and differentiation from other autofluorescent lysosomal storage bodies. Arch. Gerontol. Geriatr. 34, 169-184. https://doi.org/10.1016/S01674943(02)00005-5

Katz, M.L., Robison, W.G., 1984. Age-related changes in the retinal pigment epithelium of pigmented rats. Exp. Eye Res. 38, 137-151. https://doi.org/https://doi.org/10.1016/0014-4835(84)90098-8

Katz, M.L., Rustad, E., Robinson, G.O., Whiting, R.E.H., Student, J.T., Coates, J.R., Narfstrom, K., 2017b. Canine neuronal ceroid lipofuscinoses: Promising models for preclinical testing of therapeutic interventions. Neurobiol. Dis. 108, 277-287. https://doi.org/10.1016/j.nbd.2017.08.017

Katz, M.L., Shanker, M.J., 1989. Development of lipofuscin-like fluorescence in the retinal pigment epithelium in response to protease inhibitor treatment. Mech. Ageing Dev. 49, 23-40. https://doi.org/https://doi.org/10.1016/00476374(89)90065-1

Katz, M.L., Tecedor, L., Chen, Y., Williamson, B.G., Lysenko, E., Wininger, F. a, Young, W.M., Johnson, G.C., Whiting, R.E.H., Coates, J.R., Davidson, B.L., 2015. AAV gene transfer delays disease onset in a TPP1-deficient canine model of the late infantile form of Batten disease. Sci. Transl. Med. 7 , 313ra180-313ra180. https://doi.org/10.1126/scitranslmed.aac6191

Kemp, K., Hares, K., Mallam, E., Heesom, K.J., Scolding, N., Wilkins, A., 2010. Mesenchymal stem cell-secreted superoxide dismutase promotes cerebellar neuronal survival. J. Neurochem. 114, 1569-1580. https://doi.org/10.1111/j.1471-4159.2009.06553.x

Khasawneh, R.R., Al Sharie, A.H., Abu-El Rub, E., Serhan, A.O., Obeidat, H.N., 2019. Addressing the impact of different fetal bovine serum percentages on mesenchymal stem cells biological performance. Mol. Biol. Rep. 46, 44374441. https://doi.org/10.1007/s11033-019-04898-1

Kick, G.R., Meiman, E.J., Sabol, J.C., Whiting, R.E.H., Ota-Kuroki, J., Castaner, L.J., Jensen, C.A., Katz, M.L., 2021. Visual system pathology in a canine model of CLN5 neuronal ceroid lipofuscinosis. Exp. Eye Res. 108686. https://doi.org/https://doi.org/10.1016/j.exer.2021.108686

Kida, E., Golabek, A.A., Walus, M., Wujek, P., Kaczmarski, W., Wisniewski, K.E., 2001. Distribution of tripeptidyl peptidase I in human tissues under normal and pathological conditions. J. Neuropathol. Exp. Neurol. 60, 280-92.

Kim, H., Csaky, K.G., Gravlin, L., Yuan, P., Lutz, R.J., Bungay, P.M., Tansey, G., 
De Monasterio, F., Potti, G.K., Grimes, G., Robinson, M.R., 2006. Safety and pharmacokinetics of a preservative-free triamcinolone acetonide formulation for intravitreal administration. RETINA 26.

Kimotsuki, T., Yasuda, M., Tamahara, S., Matsuki, N., Ono, K., 2005.

Topographic analysis of flash visual evoked potentials in dogs. J. Vet. Med. Sci. 67, 869-875. https://doi.org/10.1292/jvms.67.869

Kirveskari, E., Partinen, M., Santavuori, P., 2001. Sleep and Its Disturbance in a Variant Form of Late Infantile Neuronal Ceroid Lipofuscinosis (CLNS). J. Child Neurol. 16, 707-713. https://doi.org/10.1177/088307380101601001

Klockars, T., Holmberg, V., Savukoski, M., Lander, E.S., Peltonen, L., 1999a. Transcript identification on the CLN5 region on chromosome 13q22. Hum. Genet. 105, 51-56. https://doi.org/10.1007/s004399900069

Klockars, T., Savukoski, M., Isosomppi, J., Peltonen, L., 1999b. Positional cloning of the CLN5 gene defective in the Finnish variant of the LINCL. Mol. Genet. Metab. 66, 324-328. https://doi.org/10.1006/mgme.1999.2832

Kolicheski, A., Johnson, G.S., O'Brien, D.P., Mhlanga-Mutangadura, T., Gilliam, D., Guo, J., Anderson-Sieg, T.D., Schnabel, R.D., Taylor, J.F., Lebowitz, A., Swanson, B., Hicks, D., Niman, Z.E., Wininger, F.A., Carpentier, M.C., Katz, M.L., 2016. Australian Cattle Dogs with Neuronal Ceroid Lipofuscinosis are Homozygous for a CLN5 Nonsense Mutation Previously Identified in Border Collies. J. Vet. Intern. Med. 30, 1149-1158.

https://doi.org/10.1111/jvim.13971

Kollmann, K., Uusi-Rauva, K., Scifo, E., Tyynelä, J., Jalanko, A., Braulke, T., 2013. Cell biology and function of neuronal ceroid lipofuscinosis-related proteins. Biochim. Biophys. Acta - Mol. Basis Dis. 1832, 1866-1881. https://doi.org/10.1016/j.bbadis.2013.01.019

Kotterman, M.A., Schaffer, D. V., 2014. Engineering adeno-associated viruses for clinical gene therapy. Nat. Rev. Genet. 15, 445-51. https://doi.org/10.1038/nrg3742

Kousi, M., Lehesjoki, A.-E., Mole, S.E., 2012. Update of the mutation spectrum and clinical correlations of over 360 mutations in eight genes that underlie the neuronal ceroid lipofuscinoses. Hum Mutat 33, 42-63. https://doi.org/10.1002/humu.21624

Krzystolik, M.G., Afshari, M.A., Adamis, A.P., Gaudreault, J., Gragoudas, E.S., Michaud, N.A., Li, W., Connolly, E., O’Neill, C.A., Miller, J.W., 2002.

Prevention of experimental choroidal neovascularization with intravitreal antivascular endothelial growth factor antibody fragment. Arch. Ophthalmol. 120, 338-346. https://doi.org/10.1001/archopht.120.3.338

Ku, J.H., Ali, A., Suhler, E.B., Choi, D., Rosenbaum, J.T., 2012. Characteristics and visual outcome of patients with retinal vasculitis. Arch. Ophthalmol. 130, 1261-1266. https://doi.org/10.1001/archophthalmol.2012.1596 
Labrador-Velandia, S., Alonso-Alonso, M.L., Alvarez-Sanchez, S., GonzálezZamora, J., Carretero-Barrio, I., Pastor, J.C., Fernandez-Bueno, I., Srivastava, G.K., 2016. Mesenchymal stem cell therapy in retinal and optic nerve diseases: An update of clinical trials. World J. Stem Cells 8, 376. https://doi.org/10.4252/wjsc.v8.i11.376

Lam, P.K., Lo, A.W.I., Wang, K.K.W., Lau, H.C.H., Leung, K.K.C., Li, K.T.C., Lai, P.B.S., Poon, W.S., 2013. Transplantation of mesenchymal stem cells to the brain by topical application in an experimental traumatic brain injury model. J. Clin. Neurosci. 20, 306-309. https://doi.org/10.1016/j.jocn.2012.03.028

Larkin, H., Ribeiro, M.G., Lavoie, C., 2013. Topology and Membrane Anchoring of the Lysosomal Storage Disease-Related Protein CLN5. Hum. Mutat. 34, 1688-1697. https://doi.org/10.1002/humu.22443

Lebrun, A.-H., Storch, S., Rüschendorf, F., Schmiedt, M.-L., Kyttälä, A., Mole, S.E., Kitzmüller, C., Saar, K., Mewasingh, L.D., Boda, V., Kohlschütter, A., Ullrich, K., Braulke, T., Schulz, A., 2009. Retention of lysosomal protein CLN5 in the endoplasmic reticulum causes neuronal ceroid lipofuscinosis in Asian Sibship. Hum. Mutat. 30, E651-E661.

https://doi.org/10.1002/humu.21010

Lechanteur, C., Briquet, A., Giet, O., Delloye, O., Baudoux, E., Beguin, Y., 2016. Clinical-scale expansion of mesenchymal stromal cells: a large banking experience. J. Transl. Med. 14. https://doi.org/10.1186/s12967-016-0892-y

Lee, J.-Y., Shin, J.-M., Yeum, C.E., Chae, G.T., Chun, M.-H., Oh, S.-J., 2012. Intravitreal delivery of mesenchymal stem cells loaded onto hydrogel affects the regulatory expression of endogenous NGF and BDNF in ischemic rat retina. Tissue Eng. Regen. Med. 9, 249-258. https://doi.org/10.1007/s13770-012-0355-3

Lee, R.W., Nicholson, L.B., Sen, H.N., Chan, C.C., Wei, L., Nussenblatt, R.B., Dick, A.D., 2014. Autoimmune and autoinflammatory mechanisms in uveitis. Semin. Immunopathol. 36, 581-594. https://doi.org/10.1007/s00281-0140433-9

Leinonen, H., Keksa-Goldsteine, V., Ragauskas, S., Kohlmann, P., Singh, Y., Savchenko, E., Puranen, J., Malm, T., Kalesnykas, G., Koistinaho, J., Tanila, H., Kanninen, K.M., 2017. Retinal Degeneration in A Mouse Model of CLN5 Disease Is Associated with Compromised Autophagy. Sci. Rep. 7, 1-12. https://doi.org/10.1038/s41598-017-01716-1

Lewis, G., Morrill, A.M., Conway-Allen, S.L., Kim, B., 2019. Review of Cerliponase Alfa: Recombinant Human Enzyme Replacement Therapy for Late-Infantile Neuronal Ceroid Lipofuscinosis Type 2. J. Child Neurol. 2-7. https://doi.org/10.1177/0883073819895694

Lin, L., Lobel, P., 2001. Enzyme-replacement therapy in late infantile neuronal ceroid lipofuscinosis. Biochem. J. 55, 49-55. 
Lin, L., Sohar, I., Lackland, H., Lobel, P., 2001. The human CLN2 protein/tripeptidyl-peptidase I is a serine protease that autoactivates at acidic pH. J. Biol. Chem. 276, 2249-2255. https://doi.org/10.1074/jbc.M008562200

Liu, G.T., 2003. Chapter 6 - Disorders of the Eyes and Eyelids, in: Samuels, M.A., Feske, S.K. (Eds.), Office Practice of Neurology. Churchill Livingstone, Philadelphia, Pennsylvania, pp. 35-69. https://doi.org/10.1016/B0-44306557-8/50008-3

Löbel, U., Sedlacik, J., Nickel, M., Lezius, S., Fiehler, J., Nestrasil, I., Kohlschütter, A., Schulz, A., 2016. Volumetric description of brain atrophy in neuronal ceroid lipofuscinosis 2: Supratentorial gray matter shows uniform disease progression. Am. J. Neuroradiol. 37, 1938-1943. https://doi.org/10.3174/ajnr.A4816

Lorenz, M.D., Coates, J.R., Kent, M., 2011. Neurologic History, Neuroanatomy, and Neurologic Examination. Handb. Vet. Neurol. 2-36. https://doi.org/10.1016/b978-1-4377-0651-2.10001-3

Lyly, A., von Schantz, C., Heine, C., Schmiedt, M.L., Sipilä, T., Jalanko, A., Kyttälä, A., 2009. Novel interactions of CLN5 support molecular networking between Neuronal Ceroid Lipofuscinosis proteins. BMC Cell Biol. 10, 1-11. https://doi.org/10.1186/1471-2121-10-83

Maguire, A.M., Russell, S., Wellman, J.A., Chung, D.C., Yu, Z.-F., Tillman, A., Wittes, J., Pappas, J., Elci, O., Marshall, K.A., McCague, S., Reichert, H., Davis, M., Simonelli, F., Leroy, B.P., Wright, J.F., High, K.A., Bennett, J., 2019. Efficacy, Safety, and Durability of Voretigene Neparvovec-rzyl in RPE65 Mutation-Associated Inherited Retinal Dystrophy. Ophthalmology 126, 1273-1285. https://doi.org/10.1016/j.ophtha.2019.06.017

Maguire, M.G., Martin, D.F., Ying, G., Jaffe, G.J., Daniel, E., Grunwald, J.E., Toth, C.A., Ferris, F.L., Fine, S.L., 2016. Five-Year Outcomes with AntiVascular Endothelial Growth Factor Treatment of Neovascular Age-Related Macular Degeneration. Ophthalmology 123, 1751-1761. https://doi.org/10.1016/j.ophtha.2016.03.045

Malysz-Cymborska, I., Golubczyk, D., Kalkowski, L., Kwiatkowska, J., Zawadzki, M., Głodek, J., Holak, P., Sanford, J., Milewska, K., Adamiak, Z., Walczak, P., Janowski, M., 2021. Intra-arterial transplantation of stem cells in large animals as a minimally-invasive strategy for the treatment of disseminated neurodegeneration. Sci. Rep. 11, 1-10. https://doi.org/10.1038/s41598-02185820-3

Mamo, A., Jules, F., Dumaresq-Doiron, K., Costantino, S., Lefrancois, S., 2012. The Role of Ceroid Lipofuscinosis Neuronal Protein 5 (CLN5) in Endosomal Sorting. Mol. Cell. Biol. 32, 1855-1866. https://doi.org/10.1128/mcb.0672611

Mareschi, K., Rustichelli, D., Calabrese, R., Gunetti, M., Sanavio, F., Castiglia, 
S., Risso, A., Ferrero, I., Tarella, C., Fagioli, F., 2012. Multipotent mesenchymal stromal stem cell expansion by plating whole bone marrow at a low cellular density: A more advantageous method for clinical use. Stem Cells Int. 2012. https://doi.org/10.1155/2012/920581

Markham, A., 2017. Cerliponase Alfa: First Global Approval. Drugs 77, 12471249. https://doi.org/10.1007/s40265-017-0771-8

Marticorena, J., Romano, V., Gómez-Ulla, F., 2012. Sterile endophthalmitis after intravitreal injections. Mediators Inflamm. 2012. https://doi.org/10.1155/2012/928123

May, A., Nimtschke, U., May, C.A., 2009. The architecture of the mouse ciliary processes and their changes during retinal degeneration. Exp. Eye Res. 88, 561-565. https://doi.org/10.1016/j.exer.2008.11.006

May, C.A., Lütjen-Drecoll, E., Narfström, K., 2005. Morphological Changes in the Anterior Segment of the Abyssinian Cat Eye with Hereditary Rod-Cone Degeneration. Curr. Eye Res. 30, 855-862. https://doi.org/10.1080/02713680591006219

Melville, S.A., Wilson, C.L., Chiang, C.S., Studdert, V.P., Lingaas, F., Wilton, A.N., 2005. A mutation in canine CLN5 causes neuronal ceroid lipofuscinosis in Border collie dogs is. Genomics 86, 287-294. https://doi.org/10.1016/j.ygeno.2005.06.005

Mendell, J.R., Al-Zaidy, S.A., Lehman, K.J., McColly, M., Lowes, L.P., Alfano, L.N., Reash, N.F., lammarino, M.A., Church, K.R., Kleyn, A., Meriggioli, M.N., Shell, R., 2021. Five-Year Extension Results of the Phase 1 START Trial of Onasemnogene Abeparvovec in Spinal Muscular Atrophy. JAMA Neurol. 43205, 1-8. https://doi.org/10.1001/jamaneurol.2021.1272

Mitchell, N.L., Russell, K.N., Wellby, M.P., Wicky, H.E., Schoderboeck, L., Barrell, G.K., Melzer, T.R., Gray, S.J., Hughes, S.M., Palmer, D.N., 2018. Longitudinal In Vivo Monitoring of the CNS Demonstrates the Efficacy of Gene Therapy in a Sheep Model of CLN5 Batten Disease. Mol. Ther. 26, 2366-2378. https://doi.org/10.1016/j.ymthe.2018.07.015

Moharir, A., Peck, S.H., Budden, T., Lee, S.Y., 2013. The Role of NGlycosylation in Folding, Trafficking, and Functionality of Lysosomal Protein CLN5. PLoS One 8, e74299. https://doi.org/10.1371/journal.pone.0074299

Mole, S.E., 2004. The Genetic Spectrum of Human Neuronal Ceroidlipofuscinoses. Brain Pathol. 14, 70-76. https://doi.org/10.1111/j.17503639.2004.tb00500.x

Molleda, J.M., Tardón, R.H., Gallardo, J.M., Martín-Suárez, E.M., 2008. The ocular effects of intravitreal triamcinolone acetonide in dogs. Vet. J. 176, 326-332. https://doi.org/10.1016/j.tvjl.2007.02.031

Morgan, B.R., Coates, J.R., Johnson, G.C., Shelton, G.D., Katz, M.L., 2014. 
Characterization of thoracic motor and sensory neurons and spinal nerve roots in canine degenerative myelopathy, a potential disease model of amyotrophic lateral sclerosis. J. Neurosci. Res. 92, 531-541. https://doi.org/10.1002/jnr.23332

Morita, E., Watanabe, Y., Ishimoto, M., Nakano, T., Kitayama, M., Yasui, K., Fukada, Y., Doi, K., Karunaratne, A., Murrell, W.G., Sutharsan, R., MackaySim, A., Hata, Y., Nakashima, K., 2008. A novel cell transplantation protocol and its application to an ALS mouse model. Exp. Neurol. 213, 431-438. https://doi.org/10.1016/j.expneurol.2008.07.011

Mowat, F., Gornik, K., Dinculescu, A., Boye, S., Hauswirth, W., Petersen-Jones, S., Bartoe, J., 2014. Tyrosine capsid-mutant AAV vectors for gene delivery to the canine retina from a subretinal or intravitreal approach. Gene Ther. 21, 96-105. https://doi.org/10.1007/s10995-015-1800-4.Alcohol

Mowat, F.M., Petersen-Jones, S.M., Williamson, H., Williams, D.L., Luthert, P.J., Ali, R.R., Bainbridge, J.W., 2008. Topographical characterization of cone photoreceptors and the area centralis of the canine retina. Mol. Vis. 14, 2518-2527.

Murray, S.J., Russell, K.N., Melzer, T.R., Gray, S.J., Heap, S.J., Palmer, D.N., Mitchell, N.L., 2021. Intravitreal gene therapy protects against retinal dysfunction and degeneration in sheep with CLN5 Batten disease. Exp. Eye Res. 108600. https://doi.org/10.1016/j.exer.2021.108600

Narfström, K., Katz, M.L., Bragadottir, R., Seeliger, M., Boulanger, A., Redmond, T.M., Caro, L., Lai, C.-M., Rakoczy, P.E., 2003. Functional and Structural Recovery of the Retina after Gene Therapy in the RPE65 Null Mutation Dog. Investig. Opthalmology Vis. Sci. 44, 1663. https://doi.org/10.1167/iovs.020595

Narfström, K., Vaegan, Katz, M., Bragadottir, R., Rakoczy, E.P., Seeliger, M., 2005. Assessment of Structure and Function Over a 3-year Period after Gene Transfer in RPE65-/- dogs. Doc. Ophthalmol. 111, 39-48. https://doi.org/10.1007/s10633-005-3159-0

Naso, M.F., Tomkowicz, B., Perry, W.L., Strohl, W.R., 2017. Adeno-Associated Virus (AAV) as a Vector for Gene Therapy. BioDrugs 31, 317-334. https://doi.org/10.1007/s40259-017-0234-5

Nathwani, A.C., Reiss, U., Tuddenham, E., Chowdary, P., McIntosh, J., Riddell, A., Pie, J., Mahlangu, J.N., Recht, M., Shen, Y.-M., Halka, K.G., Meagher, M.M., Nienhuis, A.W., Davidoff, A.M., Mangles, S., Morton, C.L., Junfang, Z., Radulescu, V.C., 2018. Adeno-Associated Mediated Gene Transfer for Hemophilia B:8 Year Follow up and Impact of Removing "Empty Viral Particles" on Safety and Efficacy of Gene Transfer. Blood 132, 491-491. https://doi.org/10.1182/blood-2018-99-118334

NCL Mutation and Patient Database [WWW Document], n.d. URL 
https://www.ucl.ac.uk/ncl-disease/ncl-resource-gateway-batten-

disease/mutation-and-patient-database/mutation-and-patient-datasheets-4 (accessed 5.3.21).

Nguyen, G.N., Everett, J.K., Kafle, S., Roche, A.M., Raymond, H.E., Leiby, J., Wood, C., Assenmacher, C.-A., Merricks, E.P., Long, C.T., Kazazian, H.H., Nichols, T.C., Bushman, F.D., Sabatino, D.E., 2021. A long-term study of AAV gene therapy in dogs with hemophilia $A$ identifies clonal expansions of transduced liver cells. Nat. Biotechnol. 39, 47-55. https://doi.org/10.1038/s41587-020-0741-7

Nickel, M., Simonati, A., Jacoby, D., Lezius, S., Kilian, D., Van de Graaf, B., Pagovich, O.E., Kosofsky, B., Yohay, K., Downs, M., Slasor, P., Ajayi, T., Crystal, R.G., Kohlschütter, A., Sondhi, D., Schulz, A., 2018. Disease characteristics and progression in patients with late-infantile neuronal ceroid lipofuscinosis type 2 (CLN2) disease: an observational cohort study. Lancet Child Adolesc. Heal. 2, 582-590. https://doi.org/10.1016/S23524642(18)30179-2

Oner, A., Gonen, Z.B., Sinim, N., Cetin, M., Ozkul, Y., 2016. Subretinal adipose tissue-derived mesenchymal stem cell implantation in advanced stage retinitis pigmentosa: a phase I clinical safety study. Stem Cell Res. Ther. 7, 1-12. https://doi.org/10.1186/s13287-016-0432-y

Orlin, A., Sondhi, D., Witmer, M.T., Wessel, M.M., Mezey, J.G., Kaminsky, S.M., Hackett, N.R., Yohay, K., Kosofsky, B., Souweidane, M.M., Kaplitt, M.G., D’Amico, D.J., Crystal, R.G., Kiss, S., 2013. Spectrum of Ocular Manifestations in CLN2-Associated Batten (Jansky-Bielschowsky) Disease Correlate with Advancing Age and Deteriorating Neurological Function. PLoS One 8, 1-13. https://doi.org/10.1371/journal.pone.0073128

Özmert, E., Arslan, U., 2020. Management of retinitis pigmentosa by Wharton's jelly-derived mesenchymal stem cells: Prospective analysis of 1-year results. Stem Cell Res. Ther. 11, 1-16. https://doi.org/10.1186/s13287-020-01870-w

Padhy, S.K., Takkar, B., Narayanan, R., Venkatesh, P., Jalali, S., 2020. Voretigene neparvovec and gene therapy for leber's congenital amaurosis: Review of evidence to date. Appl. Clin. Genet. 13, 179-208. https://doi.org/10.2147/TACG.S230720

Palmer, D.N., Barry, L.A., Tyynelä, J., Cooper, J.D., 2013. NCL disease mechanisms. Biochim. Biophys. Acta - Mol. Basis Dis. 1832, 1882-1893. https://doi.org/10.1016/j.bbadis.2013.05.014

Pardridge, W.M., 2005. The blood-brain barrier: Bottleneck in brain drug development. NeuroRx 2, 3-14. https://doi.org/10.1602/neurorx.2.1.3

Park, S.J., Choi, Y., Na, Y.M., Hong, H.K., Park, J.Y., Park, K.H., Chung, J.Y., Woo, S.J., 2016. Intraocular pharmacokinetics of intravitreal aflibercept (Eylea) in a rabbit model. Investig. Ophthalmol. Vis. Sci. 57, 2612-2617. 
https://doi.org/10.1167/iovs.16-19204

Pavlou, M., Schön, C., Occelli, L.M., Rossi, A., Meumann, N., Boyd, R.F., Bartoe, J.T., Siedlecki, J., Gerhardt, M.J., Babutzka, S., Bogedein, J., Wagner, J.E., Priglinger, S.G., Biel, M., Michalakis, S., 2021. Novel AAV capsids for intravitreal gene therapy of photoreceptor disorders 1-19.

https://doi.org/10.15252/emmm.202013392

Perentos, N., Martins, A.Q., Cumming, R.J.M., Mitchell, N.L., Palmer, D.N., Sawiak, S.J., Morton, A.J., 2016. An EEG Investigation of Sleep Homeostasis in Healthy and CLN5 Batten Disease Affected Sheep. J. Neurosci. 36, 8238-8249. https://doi.org/10.1523/JNEUROSCI.429515.2016

Perentos, N., Martins, A.Q., Watson, T.C., Bartsch, U., Mitchell, N.L., Palmer, D.N., Jones, M.W., Jennifer Morton, A., 2015. Translational neurophysiology in sheep: Measuring sleep and neurological dysfunction in CLN5 Batten disease affected sheep. Brain 138, 862-874. https://doi.org/10.1093/brain/awv026

Petersen, B., Handwerker, M., Huppertz, H., 1996. Neuroradiological findings in classical late infantile neuronal ceroid-lipofuscinosis. 1Pediatric Neurol. 15, 344-347.

Peyvandi, F., Garagiola, I., 2019. Clinical advances in gene therapy updates on clinical trials of gene therapy in haemophilia. Haemophilia 25, 738-746. https://doi.org/10.1111/hae.13816

Pineda-Trujillo, N., Cornejo, W., Carrizosa, J., Wheeler, R.B., Múnera, S., Valencia, A., Agudelo-Arango, J., Cogollo, A., Anderson, G., Bedoya, G., Mole, S.E., Ruíz-Linares, A., 2005. A CLN5 mutation causing an atypical neuronal ceroid lipofuscinosis of juvenile onset. Neurology 64, 740-742. https://doi.org/10.1212/01.WNL.0000151974.44980.F1

Ponce, R., Abad, L., Amaravadi, L., Gelzleichter, T., Gore, E., Green, J., Gupta, S., Herzyk, D., Hurst, C., Ivens, I.A., Kawabata, T., Maier, C., Mounho, B., Rup, B., Shankar, G., Smith, H., Thomas, P., Wierda, D., 2009. Immunogenicity of biologically-derived therapeutics: Assessment and interpretation of nonclinical safety studies. Regul. Toxicol. Pharmacol. 54, 164-182. https://doi.org/10.1016/j.yrtph.2009.03.012

Radhakrishnan, K., Sonali, N., Moreno, M., Nirmal, J., Fernandez, A.A., Venkatraman, S., Agrawal, R., 2017. Protein delivery to the back of the eye: barriers, carriers and stability of anti-VEGF proteins. Drug Discov. Today 22, 416-423. https://doi.org/10.1016/j.drudis.2016.10.015

Radke, J., Stenzel, W., Goebel, H.H., 2015. Human NCL Neuropathology. Biochim. Biophys. Acta - Mol. Basis Dis. 1852, 2262-2266. https://doi.org/10.1016/j.bbadis.2015.05.007

Ramachandran, P.S., Lee, V., Wei, Z., Song, J.Y., Casal, G., Cronin, T., Willett, 
K., Huckfeldt, R., Morgan, J.I.W., Aleman, T.S., Maguire, A.M., Bennett, J., 2017. Evaluation of Dose and Safety of AAV7m8 and AAV8BP2 in the NonHuman Primate Retina. Hum. Gene Ther. 28, 154-167.

https://doi.org/10.1089/hum.2016.111

Rietdorf, K., Coode, E.E., Schulz, A., Wibbeler, E., Bootman, M.D., Ostergaard, J.R., 2020. Cardiac pathology in neuronal ceroid lipofuscinoses (NCL): More than a mere co-morbidity. Biochim. Biophys. Acta - Mol. Basis Dis. 1866, 165643. https://doi.org/10.1016/j.bbadis.2019.165643

Rigaudière, F., Nasser, H., Pichard-Oumlil, S., Delouvrier, E., Lopez-Hernandez, E., Milani, P., Auvin, S., Delanoë, C., 2021. Evolution of the retinal function by flash-ERG in one child suffering from neuronal ceroid lipofuscinosis CLN2 treated with cerliponase alpha: case report. Doc. Ophthalmol. https://doi.org/10.1007/s10633-021-09825-z

Russell, K.N., Mitchell, N.L., Anderson, N.G., Bunt, C.R., Wellby, M.P., Melzer, T.R., Barrell, G.K., Palmer, D.N., 2018. Computed tomography provides enhanced techniques for longitudinal monitoring of progressive intracranial volume loss associated with regional neurodegeneration in ovine neuronal ceroid lipofuscinoses. Brain Behav. 8, 1-8. https://doi.org/10.1002/brb3.1096

Russell, S., Bennett, J., Wellman, J.A., Chung, D.C., Yu, Z.F., Tillman, A., Wittes, J., Pappas, J., Elci, O., McCague, S., Cross, D., Marshall, K.A., Walshire, J., Kehoe, T.L., Reichert, H., Davis, M., Raffini, L., George, L.A., Hudson, F.P., Dingfield, L., Zhu, X., Haller, J.A., Sohn, E.H., Mahajan, V.B., Pfeifer, W., Weckmann, M., Johnson, C., Gewaily, D., Drack, A., Stone, E., Wachtel, K., Simonelli, F., Leroy, B.P., Wright, J.F., High, K.A., Maguire, A.M., 2017. Efficacy and safety of voretigene neparvovec (AAV2-hRPE65v2) in patients with RPE65-mediated inherited retinal dystrophy: a randomised, controlled, open-label, phase 3 trial. Lancet 390, 849-860. https://doi.org/10.1016/S0140-6736(17)31868-8

Sanders, D.N., Kanazono, S., Coates, J.R., Johnson, G.S., Johnson, G.C., Narfstrom, K., O'Brien, D.P., Katz, M.L., 2010. Cognitive Decline in a Dog Model for an Inherited Neurodegenerative Disease Using T-Maze Performance. J. Vet. Behav. 5, 154. https://doi.org/10.1016/j.jveb.2009.12.020

Sanders, D.N., Kanazono, S., Wininger, F.A., Whiting, R.E.H., Flournoy, C.A., Coates, J.R., Castaner, L.J., O'Brien, D.P., Katz, M.L., 2011. A reversal learning task detects cognitive deficits in a Dachshund model of late-infantile neuronal ceroid lipofuscinosis. Genes, Brain Behav. 10, 798-804. https://doi.org/10.1111/j.1601-183X.2011.00718.x

Sands, M.S., Davidson, B.L., 2006. Gene therapy for lysosomal storage diseases. Mol. Ther. 13, 839-849. https://doi.org/10.1016/j.ymthe.2006.01.006

Santavuori, P., Rapola, J., Nuutila, A., Raininko, R., Lappi, M., Launes, J., Herva, 
R., Sainio, K., 1991. The Spectrum of Jansky-Bielschowsky Disease. Neuropediatrics 22, 92-96. https://doi.org/10.1055/s-2008-1071423

Santavuori, P., Rapola, J., Sainio, K., Raitta, C., 1982. A Variant of JanskyBielschowsky Disease. Neuropediatrics 13, 135-141. https://doi.org/10.1055/s-2008-1059612

Santavuori, P., Vanhanen, S.L., Autti, T., 2001. Clinical and neuroradiological diagnostic aspects of neuronal ceroid lipofuscinoses disorders. Eur. J. Paediatr. Neurol. 5, 157-161. https://doi.org/10.1053/ejpn.2000.0454

Satarian, L., Nourinia, R., Safi, S., Kanavi, M.R., Jarughi, N., Daftarian, N., Arab, L., Aghdami, N., Ahmadieh, H., Baharvand, H., 2017. Intravitreal injection of bone marrow mesenchymal stem cells in patients with advanced retinitis pigmentosa; A safety study. J. Ophthalmic Vis. Res. 12, 58-64. https://doi.org/10.4103/2008-322X.200164

Savukoski, M., Klockars, T., Holmberg, V., Santavuori, P., Lander, E.S., Peltonen, L., 1998. CLN5, a novel gene encoding a putative transmembrane protein mutated in Finnish variant late infantile neuronal ceroid lipofuscinosis. Nat. Genet. 19, 286-288. https://doi.org/10.1038/975

Schmiedt, M.L., Bessa, C., Heine, C., Ribeiro, M.G., Jalanko, A., Kyttälä, A., 2010. The neuronal ceroid lipofuscinosis protein CLN5: New insights into cellular maturation, transport, and consequences of mutations. Hum. Mutat. 31, 356-365. https://doi.org/10.1002/humu.21195

Schochet, S.S., Font, R.L., Morris, H.M., 1980. Jansky-Bielschowsky Form of Neuronal Ceroid-Lipofuscinosis: Ocular Pathology of the Batten-Vogt Syndrome. Arch Opthalmol 98, 1083-1088.

Schreckenberger, M., Eichhorn, M., Gottanka, J., Döbig, C., Lütjen-Drecoll, E., 1994. Altered Proportions of RCS-rat Eyes. Exp. Eye Res. 59, 409-416. https://doi.org/10.1006/exer.1994.1125

Schulz, A., Ajayi, T., Specchio, N., de Los Reyes, E., Gissen, P., Ballon, D., Dyke, J.P., Cahan, H., Slasor, P., Jacoby, D., Kohlschütter, A., 2018. Study of Intraventricular Cerliponase Alfa for CLN2 Disease. N. Engl. J. Med. 378, 1898-1907. https://doi.org/10.1056/NEJMoa1712649

Schwering, C., Wibbeler, E., Miriam, N., Schulz, A., 2020. Development of the "Hamburg best practice guidelines for ICV-enzyme replacement therapy (ERT) in CLN2 disease" based on 5 years treatment experience in 48 patients. Mol. Genet. Metab. 129, S146. https://doi.org/10.1016/j.ymgme.2019.11.385

Seehafer, S.S., Pearce, D.A., 2006. You say lipofuscin, we say ceroid: defining autofluorescent storage material. Neurobiol Aging 27, 576-588. https://doi.org/10.1016/j.neurobiolaging.2005.12.006

Selden, N.R., Al-Uzri, A., Huhn, S.L., Koch, T.K., Sikora, D.M., Nguyen-Driver, 
M.D., Guillaume, D.J., Koh, J.L., Gultekin, S.H., Anderson, J.C., Vogel, H., Sutcliffe, T.L., Jacobs, Y., Steiner, R.D., 2013. Central nervous system stem cell transplantation for children with neuronal ceroid lipofuscinosis. J.

Neurosurg. Pediatr. 11, 643-652.

https://doi.org/10.3171/2013.3.PEDS12397

Shichinohe, H., Kawabori, M., lijima, H., Teramoto, T., Abumiya, T., Nakayama, N., Kazumata, K., Terasaka, S., Arato, T., Houkin, K., 2017. Research on advanced intervention using novel bone marrOW stem cell (RAINBOW): A study protocol for a phase I, open-label, uncontrolled, dose-response trial of autologous bone marrow stromal cell transplantation in patients with acute ischemic stroke. BMC Neurol. 17, 1-8. https://doi.org/10.1186/s12883-0170955-6

Simonati, A., Williams, R.E., Nardocci, N., Laine, M., Battini, R., Schulz, A., Garavaglia, B., Moro, F., Pezzini, F., Santorelli, F.M., 2017. Phenotype and natural history of variant late infantile ceroid-lipofuscinosis 5. Dev. Med. Child Neurol. 59, 815-821. https://doi.org/10.1111/dmcn.13473

Sisk, D.B., Levesque, D.C., Wood, P.A., Styer, E.L., 1990. Clinical and pathologic features of ceroid lipofuscinosis in two Australian cattle dogs. J. Am. Vet. Med. Assoc. 197, 361-4.

Skerritt, G. (Ed.), 2018. King's Applied Anatomy of the Central Nervous System of Domestic Mammals, 2nd Ed. ed. John Wiley \& Sons, Inc., West Sussex, UK.

Sleat, D.E., Donnelly, R.J., Lackland, H., Liu, C.G., Sohar, I., Pullarkat, R.K., Lobel, P., 1997. Association of mutations in a lysosomal protein with classical late-infantile neuronal ceroid lipofuscinosis. Science 277, 1802-5. https://doi.org/9295267

Sleat, D.E., El-Banna, M., Sohar, I., Kim, K.H., Dobrenis, K., Walkley, S.U., Lobel, P., 2008. Residual levels of tripeptidyl-peptidase I activity dramatically ameliorate disease in late-infantile neuronal ceroid lipofuscinosis. Mol. Genet. Metab. 94, 222-233. https://doi.org/10.1016/j.ymgme.2008.01.014

Smith, R.H., 2008. Adeno-associated virus integration: Virus versus vector. Gene Ther. 15, 817-822. https://doi.org/10.1038/gt.2008.55

Squillaro, T., Peluso, G., Galderisi, U., 2016. Clinical Trials with Mesenchymal Stem Cells: An Update. Cell Transplant. 25, 829-848. https://doi.org/10.3727/096368915x689622

Steinfeld, R., Heim, P., Von Gregory, H., Meyer, K., Ullrich, K., Goebel, H.H., Kohlschütter, A., 2002. Late infantile neuronal ceroid lipofuscinosis: Quantitative description of the clinical course in patients with CLN2 mutations. Am. J. Med. Genet. 112, 347-354. https://doi.org/10.1002/ajmg.10660

Stemberger, S., Jamnig, A., Stefanova, N., Lepperdinger, G., Reindl, M., 
Wenning, G.K., 2011. Mesenchymal stem cells in a transgenic mouse model of multiple system atrophy: Immunomodulation and neuroprotection. PLoS One 6. https://doi.org/10.1371/journal.pone.0019808

Story, B.D., Miller, M.E., Bradbury, A.M., Million, E.D., Duan, D., Taghian, T., Faissler, D., Fernau, D., Beecy, S.J., Gray-Edwards, H.L., 2020. Canine Models of Inherited Musculoskeletal and Neurodegenerative Diseases. Front. Vet. Sci. 7, 1-21. https://doi.org/10.3389/fvets.2020.00080

Strain, G.M., Jackson, R.M., Tedford, B.L., 1990. Visual Evoked Potentials in the Clinically Normal Dog. J. Vet. Intern. Med. 4, 222-225. https://doi.org/10.1111/j.1939-1676.1990.tb00901.x

Studdert, V., Mitten, R., 1991. Clinical features of ceroid lipofuscinosis in border collie dogs. Aust. Vet. J. 68, 137-140. https://doi.org/10.1111/j.17510813.1991.tb03156.x

Syková, E., Rychmach, P., Drahorádová, I., Konrádová, Š., Růžičková, K., Voříšek, I., Forostyak, S., Homola, A., Bojar, M., 2017. Transplantation of mesenchymal stromal cells in patients with amyotrophic lateral sclerosis: Results of phase I/lla clinical trial. Cell Transplant. 26, 647-658. https://doi.org/10.3727/096368916X693716

Tracy, C.J., Whiting, R.E.H., Pearce, J.W., Williamson, B.G., Vansteenkiste, D.P., Gillespie, L.E., Castaner, L.J., Bryan, J.N., Coates, J.R., Jensen, C.A., Katz, M.L., 2016. Intravitreal implantation of TPP1-transduced stem cells delays retinal degeneration in canine CLN2 neuronal ceroid lipofuscinosis. Exp. Eye Res. 152, 77-87. https://doi.org/10.1016/j.exer.2016.09.003

Trapani, I., Auricchio, A., 2018. Seeing the Light after 25 Years of Retinal Gene Therapy. Trends Mol. Med. 24, 669-681. https://doi.org/10.1016/j.molmed.2018.06.006

Trivizki, O., Schwartz, S., Negri, N., Loewenstein, A., Rabina, G., Shulman, S., 2018. Noninfectious Inflammatory Response following Intravitreal Bevacizumab Injections: Description of a Cluster of Cases in Two Centers and a Review of the Literature. Ophthalmologica 240, 163-166. https://doi.org/10.1159/000492681

Tyynelä, J., Cooper, J.D., Khan, M.N., Shemilt, S.J.A., Haltia, M., 2004. Hippocampal pathology in the human neuronal ceroid-lipofuscinoses: Distinct patterns of storage deposition, neurodegeneration and glial activation. Brain Pathol. 14, 349-357. https://doi.org/10.1111/j.17503639.2004.tb00077.x

Tyynelä, J., Suopanki, J., Santavuori, P., Baumann, M., Haltia, M., 1997. Variant Late Infantile Neuronal Ceroid-lipofuscinosis. J. Neuropathol. Exp. Neurol. 56, 369-375. https://doi.org/10.1097/00005072-199704000-00005

Uccelli, A., Benvenuto, F., Laroni, A., Giunti, D., 2011. Neuroprotective features of mesenchymal stem cells. Best Pract. Res. Clin. Haematol. 24, 59-64. 
https://doi.org/10.1016/j.beha.2011.01.004

Uccelli, A., Moretta, L., Pistoia, V., 2008. Mesenchymal stem cells in health and disease. Nat Rev Immunol 8, 726-736. https://doi.org/10.1038/nri2395

Uvebrant, P., Hagberg, B., 1997. Neuronal Ceroid Lipofuscinoses in Scandinavia: Epidemiology and Clinical Pictures. Neuropediatrics 28, 6-8. https://doi.org/10.1055/s-2007-973654

Vandamme, C., Adjali, O., Mingozzi, F., 2017. Unraveling the Complex Story of Immune Responses to AAV Vectors Trial After Trial. Hum. Gene Ther. 28, 1061-1074. https://doi.org/10.1089/hum.2017.150

Vandenberghe, L.H., Auricchio, A., 2012. Novel adeno-associated viral vectors for retinal gene therapy. Gene Ther. 19, 162-168.

https://doi.org/10.1038/gt.2011.151

Vandenberghe, L.H., Bell, P., Maguire, A.M., Cearley, C.N., Xiao, R., Calcedo, R., Wang, L., Castle, M.J., Maguire, A.C., Grant, R., Wolfe, J.H., Wilson, J.M., Bennett, J., 2011. Dosage Thresholds for AAV2 and AAV8 Photoreceptor Gene Therapy in Monkey. Sci. Transl. Med. 3, 88ra5488ra54. https://doi.org/10.1126/scitranslmed.3002103

Vandenberghe, L.H., Bell, P., Maguire, A.M., Xiao, R., Hopkins, T.B., Grant, R., Bennett, J., Wilson, J.M., 2013. AAV9 Targets Cone Photoreceptors in the Nonhuman Primate Retina. PLoS One 8, e53463. https://doi.org/10.1371/journal.pone.0053463

Vedantham, V., Kolluru, C., Ramasamy, K., 2005. Persistent depot of triamcinolone acetonide after a single intravitreal injection. Indian $\mathrm{J}$. Ophthalmol. 53, 65-66. https://doi.org/10.4103/0301-4738.15291

Vesa, J., Chin, M.H., Oelgeschläger, K., Isosomppi, J., DellAngelica, E.C., Jalanko, A., Peltonen, L., 2002. Neuronal Ceroid Lipofuscinoses Are Connected at Molecular Level: Interaction of CLN5 Protein with CLN2 and CLN3. Mol. Biol. Cell 13, 2410-2420. https://doi.org/10.1091/mbc.e02-010031

Villani, N.A., Bullock, G., Michaels, J.R., Yamato, O., O'Brien, D.P., MhlangaMutangadura, T., Johnson, G.S., Katz, M.L., 2019. A mixed breed dog with neuronal ceroid lipofuscinosis is homozygous for a CLN5 nonsense mutation previously identified in border collies and Australian cattle dogs. Mol. Genet. Metab. 0-1. https://doi.org/10.1016/j.ymgme.2019.04.003

Vines, D., Warburton, M.J., 1998. Purification and characterisation of a tripeptidyl aminopeptidase I from rat spleen. Biochim. Biophys. Acta - Protein Struct. Mol. Enzymol. 1384, 233-242. https://doi.org/10.1016/S01674838(98)00012-0

Vines, D.J., Warburton, M.J., 1999. Classical late infantile neuronal ceroid lipofuscinosis fibroblasts are deficient in lysosomal tripeptidyl peptidase I. 
FEBS Lett. 443, 131-135. https://doi.org/10.1016/S0014-5793(98)01683-4

von Eisenhart-Rothe, P., Grubman, A., Greferath, U., Fothergill, L.J., Jobling, A.I., Phipps, J.A., White, A.R., Fletcher, E.L., Vessey, K.A., 2018. Failure of Autophagy-Lysosomal Pathways in Rod Photoreceptors Causes the Early Retinal Degeneration Phenotype Observed in Cln6nclf Mice. Invest. Ophthalmol. Vis. Sci. 59, 5082-5097. https://doi.org/10.1167/iovs.18-24757

Vuillemenot, B.R., Katz, M.L., Coates, J.R., Kennedy, D., Tiger, P., Kanazono, S., Lobel, P., Sohar, I., Xu, S., Cahayag, R., Keve, S., Koren, E., Bunting, S., Tsuruda, L.S., O'Neill, C.A., 2011. Intrathecal tripeptidyl-peptidase 1 reduces lysosomal storage in a canine model of late infantile neuronal ceroid lipofuscinosis. Mol. Genet. Metab. 104, 325-337. https://doi.org/10.1016/j.ymgme.2011.06.018

Vuillemenot, B.R., Kennedy, D., Cooper, J.D., Wong, A.M.S., Sri, S., Doeleman, T., Katz, M.L., Coates, J.R., Johnson, G.C., Reed, R.P., Adams, E.L., Butt, M.T., Musson, D.G., Henshaw, J., Keve, S., Cahayag, R., Tsuruda, L.S., O'Neill, C.A., 2015. Nonclinical evaluation of CNS-administered TPP1 enzyme replacement in canine CLN2 neuronal ceroid lipofuscinosis. Mol. Genet. Metab. 114, 281-293. https://doi.org/10.1016/j.ymgme.2014.09.004

Wakshull, E., Quarmby, V., Mahler, H.C., Rivers, H., Jere, D., Ramos, M., Szczesny, P., Bechtold-Peters, K., Masli, S., Gupta, S., 2017.

Advancements in Understanding Immunogenicity of Biotherapeutics in the Intraocular Space. AAPS J. 19, 1656-1668. https://doi.org/10.1208/s12248017-0128-y

Warrier, V., Vieira, M., Mole, S.E., 2013. Genetic basis and phenotypic correlations of the neuronal ceroid lipofusinoses. Biochim. Biophys. Acta Mol. Basis Dis. 1832, 1827-1830. https://doi.org/10.1016/j.bbadis.2013.03.017

Wavre-Shapton, S.T., Calvi, A.A., Turmaine, M., Seabra, M.C., Cutler, D.F., Futter, C.E., Mitchison, H.M., 2015. Photoreceptor phagosome processing defects and disturbed autophagy in retinal pigment epithelium of $\operatorname{Cln} 3 \Delta$ ex16 mice modelling juvenile neuronal ceroid lipofuscinosis (Batten disease). Hum. Mol. Genet. 24, 7060-7074. https://doi.org/10.1093/hmg/ddv406

Weitzman, M.D., Linden, R.M., 2011. Adeno-Associated Virus Biology, in: Snyder, R.O., Moullier, P. (Eds.), Adeno-Associated Virus: Methods and Protocols. Humana Press, New York, pp. 1-23. https://doi.org/10.1007/9781-61779-370-7

Weleber, R.G., 1998. The dystrophic retina in multisystem disorders: The electroretinogram in neuronal ceroid lipofuscinosis. Eye 12, 580-590.

Whiting, R.E.H., Jensen, C.A., Pearce, J.W., Gillespie, L.E., Bristow, D.E., Katz, M.L., 2016. Intracerebroventricular gene therapy that delays neurological disease progression is associated with selective preservation of retinal 
ganglion cells in a canine model of CLN2 disease. Exp. Eye Res. 146, 276282. https://doi.org/10.1016/j.exer.2016.03.023

Whiting, R.E.H., Narfström, K., Yao, G., Pearce, J.W., Coates, J.R., Castaner, L.J., Katz, M.L., 2013. Pupillary light reflex deficits in a canine model of late infantile neuronal ceroid lipofuscinosis. Exp. Eye Res. 116, 402-410. https://doi.org/10.1016/j.exer.2013.10.006

Whiting, R.E.H., Pearce, J.W., Castaner, L.J., Jensen, C.A., Katz, R.J., Gilliam, D.H., Katz, M.L., 2015. Multifocal retinopathy in Dachshunds with CLN2 neuronal ceroid lipofuscinosis. Exp. Eye Res. 134, 123-132. https://doi.org/10.1016/j.exer.2015.02.012

Whiting, R.E.H., Pearce, J.W., Vansteenkiste, D.P., Bibi, K., Lim, S., Robinson Kick, G., Castaner, L.J., Sinclair, J., Chandra, S., Nguyen, A., O’Neill, C.A., Katz, M.L., 2020a. Intravitreal enzyme replacement preserves retinal structure and function in canine CLN2 neuronal ceroid lipofuscinosis. Exp. Eye Res. 197, 108130. https://doi.org/10.1016/j.exer.2020.108130

Whiting, R.E.H., Robinson Kick, G., Ota-Kuroki, J., Lim, S., Castaner, L.J., Jensen, C.A., Kowal, J., Nguyen, A., Corado, C., O’Neill, C.A., Katz, M.L., 2020b. Intravitreal enzyme replacement inhibits progression of retinal degeneration in canine CLN2 neuronal ceroid lipofuscinosis. Exp. Eye Res. 197, 108135. https://doi.org/10.1016/j.exer.2020.108135

Wilkins, A., Kemp, K., Ginty, M., Hares, K., Mallam, E., Scolding, N., 2009. Human bone marrow-derived mesenchymal stem cells secrete brain-derived neurotrophic factor which promotes neuronal survival in vitro. Stem Cell Res. 3, 63-70. https://doi.org/10.1016/j.scr.2009.02.006

Williams, R.E., 2011. Appendix 1: NCL incidence and prevalence data, in: Mole, S.E., Williams, R.E., Goebel, H.H. (Eds.), The Neuronal Ceroid Lipofuscinoses (Batten Disease). Oxford University Press, Oxford, pp. 361365.

Wilson, A., Webster, A., Genever, P., 2019. Nomenclature and heterogeneity: Consequences for the use of mesenchymal stem cells in regenerative medicine. Regen. Med. 14, 595-611. https://doi.org/10.2217/rme-2018-0145

Worgall, S., Kekatpure, M. V., Heier, L., Ballon, D., Dyke, J.P., Shungu, D., Mao, X., Kosofsky, B., Kaplitt, M.G., Souweidane, M.M., Sondhi, D., Hackett, N.R., Hollmann, C., Crystal, R.G., 2007. Neurological deterioration in late infantile neuronal ceroid lipofuscinosis. Neurology 69, 521-535.

https://doi.org/10.1212/01.wnl.0000267885.47092.40

Wujek, P., Kida, E., Walus, M., Wisniewski, K.E., Golabek, A.A., 2004. NGlycosylation Is Crucial for Folding, Trafficking, and Stability of Human Tripeptidyl-peptidase I. J. Biol. Chem. 279, 12827-12839. https://doi.org/10.1074/jbc.M313173200

Xin, W., Mullen, T.E., Kiely, R., Min, J., Feng, X., Cao, Y., O’Malley, L., Shen, Y., 
Chu-Shore, C., Mole, S.E., Goebel, H.H., Sims, K., 2010. CLN5 mutations are frequent in juvenile and late-onset non-Finnish patients with NCL.

Neurology 74, 565-571. https://doi.org/10.1212/WNL.0b013e3181cff70d

Yamaguchi, Katsuhiro, Yamaguchi, Keiko, Sheedlo, H.J., Turner, J.E., 1991. Ciliary body degeneration in the royal college of surgeons dystrophic rat. Exp. Eye Res. 52, 539-548. https://doi.org/https://doi.org/10.1016/00144835(91)90055-J

Yin, L., Greenberg, K., Hunter, J.J., Dalkara, D., Kolstad, K.D., Masella, B.D., Wolfe, R., Visel, M., Stone, D., Libby, R.T., DiLoreto, D., Schaffer, D., Flannery, J., Williams, D.R., Merigan, W.H., 2011. Intravitreal injection of AAV2 transduces macaque inner retina. Investig. Ophthalmol. Vis. Sci. 52, 2775-2783. https://doi.org/10.1167/iovs.10-6250

Yousefifard, M., Nasseri Maleki, S., Askarian-Amiri, S., Vaccaro, A.R., Chapman, J.R., Fehlings, M.G., Hosseini, M., Rahimi-Movaghar, V., 2020. A combination of mesenchymal stem cells and scaffolds promotes motor functional recovery in spinal cord injury: a systematic review and metaanalysis. J. Neurosurg. Spine 32, 269-284. https://doi.org/10.3171/2019.8.SPINE19201

Zhang, C., Zhou, C., Teng, J.-J., Zhao, R.-L., Song, Y.-Q., 2009. Multiple administrations of human marrow stromal cells through cerebrospinal fluid prolong survival in a transgenic mouse model of amyotrophic lateral sclerosis. Cytotherapy 11, 299-306. https://doi.org/10.1080/14653240902806986 


\section{VITA}

Grace Robinson Kick was born as Grace O'Shea Robinson on July 18, 1992 in St. Louis, Missouri. She earned a B.S. in Psychology in 2014 from Truman State University in Kirksville, Missouri. She worked as a Research Technician in the lab of Dr. David M. Holtzman at the Washington University in St. Louis School of Medicine from 2014 to 2016 where she worked with mouse models of Alzheimer's Disease. She entered the Interdisciplinary Neuroscience Program at the University of Missouri - Columbia in 2016 supported by a Life Sciences Fellowship where she worked under the supervision of Dr. Martin Katz in the School of Medicine's Department of Ophthalmology. 